\title{
Ambartsumian's vision and further insight to key puzzles of ultra-high energy astrophysics
}

\author{
G.Ter-Kazarian*1 \\ ${ }^{1}$ Byurakan Astrophysical Observatory, 378433, Aragatsotn District, Armenia
}

\begin{abstract}
We review the Ambartsumian's cosmogony, which involves his fundamental ideas on Stellar Associations and eruptive Activity of Galactic Nuclei, where the creation process is at work. It is caused by the violent outburst events of transformations of superdense matter in supermassive compact bodies in galaxies, away from the accretion physics. We discuss the pioneering works of V.A. Armbartsumyan and G.S. Saakyan carried out at Byurakan Observatory in the earlier of 1960's towards the physics of equilibrium configurations of degenerate superdense gas of elementary particles, particularity, the hyperon configurations of stellar masses. These issues have been comprehensively developed later on by G.Ter-Kazarian in the proposed theory of distortion of space-time continuum (DSTC) at huge energies (respectively, at short distances $<0.4 \mathrm{fm}$ ), which underlies the microscopic theory of black hole (MTBH). The MTBH has further proved to be quite fruitful for ultra-high energy astrophysics. The MTBH explores the most important process of spontaneous breaking of gravitation gauge symmetry at huge energies, and thereof for that of rearrangement of vacuum state. As a corollary, MTBH has smeared out the central singularities of $\mathrm{BHs}$, and makes room for their growth and merging behavior, with implications of vital interest for high energy astrophysics.
\end{abstract}

Keywords: stellar associations-galaxy: galactic nuclei-distortion of space-time continuumblack hole physics - X-rays: binaries

Contents

1. Introduction 1

2. Ambartsumian's cosmogony 8

3. The degenerate superdense gas of elementary particles 11

4. On equilibrium configurations of superdense degenerate gas masses 13

5. Internal structure of hyperon configurations of stellar masses 13

6. The mass defect of baryon stars 15

7. Assessment of growth and merging properties of BHs within PMBH 16

8. The theory of distortion of the space-time continuum 20

9. The microscopic theory of black hole: Prelinimaries 28

10. Growth of accreting supermassive black hole seeds and neutrino radiation 33

11. On the physical nature of the source of ultraluminous X-ray pulsations 43

12. Rotating black holes in microscopic theory: the implications for periodic source $M 82 X-2$

13. A study of intermediate mass black hole-candidates 60

14. The physical outlook and concluding remarks 62

15. References 73

*gago_50@yahoo.com, Corresponding author 


\section{Introduction}

As the title of the present article promises, the central theme is the Ambartsumian's cosmogony from its historical development up to current interests. It describes the phenomenon of Activity of Galactic Nuclei, the existence of supermassive compact celestial bodies, and the implications for stellar and extragalactic high energy astrophysics, and the (at least partial) observational confirmation of this astonishing fact. Following the historical evolution of Ambartsumian's views, we hope to give prominence to his fundamental ideas, which manifests itself in the many different routes leading to spectacular astrophysical predictions. This report serves as an accessible introduction to the field and presents a compact and modern up-to-date source of reference on a well-defined topic, which can be a source of advanced teaching material. The selection of topics treated in detail (and the omission of other items) may be somewhat unusual, but we think it is justified in view of the title of this report. Wherever possible, we emphasize the mutual dependence and interplay between different subjects.

The fundamental concept of activity of galactic nuclei have a long tradition of precursors that dates back to the pioneering seminal works by V.A. Ambartsumian in 1950-1965. Among others, he was credited with outstanding contributions to stellar and extragalactic astronomy. Our primary interest in present article is rather to review the original vision of Ambartsumian on the Stellar Associations (Ambartsumian, 1947, 1949, 1954, 1955, 1958c, 1960, 1971, 1984, 1985) for analysing the observational data of highly energetic starburst processes, and the existence of, so-called, superdense prestellar D-bodies in the Universe causing a different forms of eruptive Activity of Galactic Nuclei (Ambartsumian, 1958a,b, 1961, 1962, 1965, 1966, 1968a,b), where the creation process is at work. We also review the pioneering works carried out by Armbartsumyan with his student-G.S. Saakyan (Ambartsumian \& Saakyan, 1960, 1961a,b, 1965) at Byurakan Observatory in the earlier of 1960's towards the physics of equilibrium configurations of degenerate superdense gas of elementary particles, particularity, the hyperon configurations of stellar masses. Consequently, these ideas have been further developed by G.Ter-Kazarian in his later works on the DSTC (Ter-Kazarian, 1986, 1989c, 1997, 2010, 2011, 2012, 2015) at huge energies, which underlies the MTBH (Ter-Kazarian, 1989a,b, 1990, 1991, 1992, 2001, Ter-Kazarian \& Yerknapetian, 1995) with implications of vital interest for high anergy astrophysics (Ter-Kazarian, 2014, 2015, 2016a,b, 2021a, Ter-Kazarian \& Sargsyan, 2013, Ter-Kazarian \& Shidhani, 2017, 2019, Ter-Kazarian et al., 2003, 2006, 2007). Rather, we try to impart some knowledge about the fundamental nature and structure of the physical space-time in a mostly non-technical, nevertheless hopefully precise and consistent language. We hope that even professional experts will find some new viewpoints and connections between different topics. There are also several closely related physical issues worked out in Byurakan Astrophysical Observatory, not touched upon here for brevity reasons. These issues reveal an interplay between novel aspects of geometry and high energy physics, spacetime deformation induced inertia effects, and intense radiation physics. We briefly outline these issues in our second report (Ter-Kazarian, 2021b), to which the interested reader is invited to consult for further details.

With this perspective in sight, we will proceed according to the following structure. To start with, Section 2 recounts some of the highlights behind of Ambartsumian's cosmogony. Astrophysics in the second half of the 20th century is saturated with seminal ideas of an outstanding astronomer V.A. Ambartsumian, who originated in astrophysics a new paths that expands over decades. He appreciated the empirical approach to the problems of the origin and evolution of stars and galaxies when one attempts to uncover the secrets of nature by observing the key points where they are hidden. He often claimed that we can hardly achieve this aim only by theorizing. Just this approach allowed Ambartsumian to develop the cosmogonic concept (Ambartsumian, 1947, 1949, 1954, 1955, 1958a,b,c, 1960, 1961, 1962, 1965, 1966, 1968a,b, 1971, 1984, 1985), according which the stars, different star groups, and the interstellar gas are formed from certain superdense prestellar bodies by the eruption of various quantities of matter. In order of magnitude the mass of the prestellar body must be greater than the mass of a star.

Ambartsumian also was first who applied the same arguments to what appear to be violent outbursts in the nuclei of galaxies, and called attention to the instability and activity of galactic cores and, thus, raised the issue of Activity of Nuclei of Galaxies. The nuclei determines the most important processes in the life of large galaxies, particularly, its activity is responsible for the formation of the 
spiral arms and formation of stars and the stellar clusters of spherical components. His key assumption was that every nucleous contains, as a rule, a supermassive nonstellar body along with the stellar population and gas, and, that nucleous plays an essential if not dominant role in the evolution of each galaxy. These phenomena seem to occur at different stages of development and are accompanied by corresponding changes in the nuclei. The process of formation of given type of subsystem varies under different circumstances.

Section 3 deals with a discussion of the composition of a degenerate gas with a density of order of nuclear density or higher (Ambartsumian \& Saakyan, 1960), but not in the sense of a systematic and complete, textbook-like presentation, but by focusing on some characteristic and fundamental topics within this immensely rich theory. Earlier, attempting to explain supernova explosions, Zwicki (Baade \& Zwicky, 1934, Zwicky, 1938) concluded on the possible existence of superdence states of neutron stars. The corresponding theoretical model suggested by (Oppenheimer \& Volkoff, 1939). They calculated the values of masses of neutronic stars to be falling in the rage of 0.3 to 0.7 the mass of the Sun, while radii lie within the range of 6 to $20 \mathrm{~km}$. But much earlier Landau (Landau, 1932) speculated on the possibility of superdense cores in massive stars. Cameron (Cameron, 1950) took into account further the forces of mutual repulsion between neutrons acting at close neighborhood. He has found that the masses of some configurations of neutronic stars may reach $\sim 2$ the mass of the Sun. The difference in the model context between the study (Ambartsumian \& Saakyan, 1960) and those of Oppenheimer and Volkoff is the presence of hyperons in composition of particles at high density. It points out to a possible existence of equilibrium configurations with a large number of hyperons. The temperature is assumed so low that all types of fermions are degenerate. It is shown that, with increasing density, different hyperons are successively appear and increase in number. They should be stable because of Pauli's exclusion principle. The threshold densities of different hyperons are calculated. Paradoxically, the smallest threshold density does not correspond to the $\Lambda$-hyperon, having the smallest mass at rest, but to the $\Sigma^{-}$-hyperon. This suggests that a sufficiently massive celestial body in gravitational equilibrium should consist of a hyperon core, a neutron layer, and an outer envelope with the usual composition made of electrons, protons and composite nuclei.

On these premises, we review in Section 4 the equilibrium configurations of superdense degenerate gas masses (Ambartsumian \& Saakyan, 1961a). The equilibrium configurations of highly degenerate baryonic ideal and real Fermi gases of stellar masses with density of order of density of atomic nucleus are studied.

In Section 5 we give an outline of internal structure of hyperon configurations of stellar masses with density of order of density of atomic nucleus and higher (Ambartsumian \& Saakyan, 1961b). The space metric inside the configuration deviates essentially from Euclidean metric. The total number of baryons in configuration is calculated. For large values of this number there are two solutions with different total energies. Therefore, a stable configuration should be those with a larger mass defect. The gravitational mass defect of baryon stars has been investigated. The authors undertook a treatment of the case of infinitely high density. However, the problem of the state of matter in such cases where the mean distances between particles become appreciably smaller than the radius of the pion cloud surrounding $(1.4 \mathrm{fm})$ the baryons is still is open question. In the cores are identical in all the baryons or at least in some of them, additional repulsive forces should strongly make themselves felt as soon as particles come close, as was quite correctly noted by Zel'dovich (Zel'dovich, 1959) in line with the Pauli exclusion principle. Taking this to be a valid assumption, the theory of superdense degenerate state as presented by (Ambartsumian \& Saakyan, 1961a) can no longer considered applicable for the range $N \gtrsim 10^{41} \mathrm{~cm}^{-3}$.

Section 6 offers a more detailed analysis of the absolute gravitational mass defect $\triangle M=n m_{n}-M$ of neutron configurations (Ambartsumian \& Saakyan, 1961b, 1965), where $M$ is the observed mass of the star, $n$ is the number of neutrons in it, $m_{n}$ is the neutron mass. The existence of baryon configurations with an anomalous (negative) mass defect was predicted in (Ambartsumian \& Saakyan, 1961b). The essence of the anomaly is that the mass of a superdense body is greater than the sum of the rest masses of the baryons it contains. The same subject was later discussed by Zel'dovich (Zel'dovich, 1962). He demonstrated that $M<f n m_{n}$, where $f \approx 1.5 \sqrt{g_{r r}(R)}, g_{r r}$ is the spatial component of metric tensor, and $R$ is the star radius. This estimate of the upper limit of mass does not exclude 
the possibility of the existence of equilibrium configurations with mass $M>n m_{n}$. It is shown that in the case of central densities $\rho(0)$ exceeding nuclear density by an order of magnitude, $\triangle M$ exhibits anomalous behavior. The anomaly consists in that with increase in $\rho(0)$ the mass defect decreases and, in the case of central baryon densities exceeding $10^{40} \mathrm{~cm}^{-3}$, becomes negative. This phenomenon is caused by the severe disruption of additivity of internal energy in an intense gravity field, where nonlinear effects are extremely important. The configurations with an anomalous value of the absolute mass defect should be of great importance to Ambartsumian's cosmogonic concept Ambartsumian \& Saakyan (1961b), according which stars, different star groups, and the interstellar gas are formed from certain superdense prestellar bodies by the eruption of various quantities of matter. In order to relate the behavior of baryon configurations with an anomalous value of the absolute mass defect to the concept mentioned, it is necessary to construct models of superdense prestellar bodies with masses of a much greater order of magnitude than the solar mass. This would solve in principle the problem of superdense cosmogony. However, the construction of physical models of continuous superdense prestellar bodies of great mass involves difficulties. The solution of these difficulties may possibly involve the consideration of nonstationary and nonequilibrium models. Further consideration of the problems of superdense states of baryonic configurations Ambartsumian \& Saakyan (1960, 1961a,b, 1965) would confront us with many confusing and puzzling aspects. It is obvious that the problems of the state of matter at such desirable high densities can only be tackled in the framework of a new, unknown yet, fundamental physical theory apart of standard views.

The Irony of Fate. Although both fundamental ideas of the activity of galactic nuclei and the existence of supermassive bodies in galactic centers are now commonly accepted but, by the irony of fate, with irrevocably distorted upside-down meaning that the truth is the exact opposite of these claims. There is a crucial difference between Ambartsumian's interpretation of phenomenon of activity of galactic nuclei and the views of the majority of theoreticians, which based on the accretion physics and infinite collapse as the major law of Nature. While Ambartsumian put forward unquenchable hypothesis that the primary events are the outflow of matter and emission of energy from the active superdense prestellar body in nucleus, which at the beginning was an isolated body and gradually created a galaxy around itself, the theoreticians attribute the outflow of matter and other signs of nuclear activity as secondary events to the primary event of hypothetical accretion of diffuse matter into the central mass of galaxy, which commonly believed is a supermassive black hole (SMBH), described by a phenomenological model as a peculiar repercussion of general relativity (GR).

One of the achievements of contemporary observational astrophysics is the development of a quite detailed study of the physical properties of growth and merging phenomena of astrophysical black holes, even at its earliest stages. But even thanks to the fruitful interplay between the astronomical observations, the theoretical and computational analysis, the scientific situation is, in fact, more inconsistent to day. Actually, the active galactic nuclei (AGNs) with typical bolometric luminosities $10^{45-48} \mathrm{erg} \mathrm{s}^{-1}$ are amongst the most luminous emitters in the Universe, particularly at high energies (gamma-rays) and radio wavelengths. From its historical development up to current interests, the efforts in the AGN physics have evoked the study of a major unsolved problem of how efficiently such huge energy release from compact regions of AGNs observed can be generated. This energy scale severely challenges conventional source models. The huge energy release from compact regions of AGN requires extremely high efficiency (typically $\geq 10$ per cent) of conversion of rest mass to other forms of energy. This serves for the majority of theoreticians as the main argument in favour of SMBHs, with masses of millions to billions of times the mass of the Sun, as central engines of massive AGNs. Within this scenario, a BH has been formed as an almost inevitable endpoint of the gravitational collapse of a large fraction of total mass of supermassive configuration occurring after entire burning of the whole amount of spared intrinsic energy. The BHs are fueled steadily from the thick accretion disks. Such evolutionary processes of accretion onto massive BHs as the prime energy sources have immense emissive power. The astrophysical black holes come in a wide range of masses, from $\geq 3 M_{\odot}$ for stellar mass black holes to $\sim 10^{10} M_{\odot}$ for SMBHs. Although direct evidence for existence of BHs still has been elusive, it is now recognized the BH signatures in AGNs, with the implication that $\mathrm{BH}$ and galaxy formation processes are closely linked. Demography of local galaxies suggests that most galaxies harbour quiescent SMBHs in their nuclei at the present time and that the 
mass of the hosted black hole is correlated with properties of the host bulge. The visible Universe should therefore be contained at least 100 billion SMBHs. A large number of representative models towards this are available in literature, but all they are subject to many uncertainties. Specifically, the Hubble Space Telescope measurements of stellar kinematics highlight an evidence for the ubiquity of SMBHs. However, the most important characteristics of the AGN powerhouse, the central masses and structures, and the $\mathrm{BH}$ formation and growth processes are not understood well. This issue is many-sided and fundamental, and can be settled fairly only by more investigations to be done for its better understanding.

The fact that accretion processes really take place in AGNs is already established and proven by many observations. With these observational advances, a tacit assumption of theoretical interpretation of astrophysical scenarios is a general belief reinforced by statements in textbooks, that the longstanding phenomenological model of $\mathrm{BH}(\mathrm{PMBH})$ is capable to describe the growth and merging behavior of accreting BHs. Even though the PMBH, namely, the most general Kerr-Newman black hole model, with parameters of mass $(M)$, angular momentum $(J)$ and charge $(Q)$ (still has to put in by hand), is being among the most significant advances in astrophysics, it is rather surprising that this model is routinely used to explore the growth and merging behavior of BHs. Altogether based on this belief, the question then arises: What procedure is in fact employed by the astronomers in the course of reaching the conclusion while estimating a growth of energy-mass of astrophysical $\mathrm{BH}$ ? The following stepwise properties are commonly attributed to above procedure:

(i) At first, from observations of surroundings of the $\mathrm{BH}$, the astronomers by means of simulation estimated a total amount of the outside mass that potentially can be swallowed driven by an accretion onto BH.

(ii) Secondly, this amount of mass, without any substantiation, is simply accepted as a real physical measure of growth of energy-mass of the astrophysical BH.

Although arguably all these reasonings seemed appealing and attractive, nevertheless there is no convincing reason to rely on a validity of such procedure and, therefore, we do not share this view. Such beliefs are suspect and should be critically re-examined. First among them is the fact that observed time-scales for flux variations of some objects are inconsistent with contemporary black hole accretion models. That is, on the basis of the diagram of the minimum variability time-scale versus the bolometric luminosity for 60 sources it has been shown that, in spite of auxiliary assumption of asymmetric emission geometry, a few BL Lac objects - B2 $1308+72$, 3C 66A, OJ 287, AO $0235+$ 16 and Quasars - 3C 345, 3C 446, 3C 454.3, LB 9743 remained in forbidden zone (particularly the three of them) (Bassani et al., 1983a,b), namely their observed sizes appeared to be less than the sizes of corresponding spheres of the event horizon. In many of the more recent papers (Ter-Kazarian, 1989a,b, 1990, 1991, 1992, 2001, Ter-Kazarian \& Yerknapetian, 1995, Ter-Kazarian et al., 2003) (and references therein) it has repeatedly been recognized that the PMBHs, at least at their current state of development, are quite incapable of making predictions of their growth and merging behavior.

In the framework of PMBHs there is no provision for growth and merging behavior of BHs because of the nasty inherent appearance of $\mathrm{BH}$ singularities, and that if the infinite collapse to the singularity inside the $\mathrm{BH}$ is accepted as a legitimate feature of Nature. Certainly, during a super-increasing of total mass of configuration one undoubtedly will arrive (irrelevant to gravitational theory in use) to a critical turning point of relativistic collapse, beyond which the gravitational forces of compression prevail over all the other forces. Than it is enough to add from the outside a small amount of energy near-by the critical point in order to begin a process of irresistible infinite catastrophic compression of configuration under the pressure of grand forces. This certainly inhibits one to answer quantitatively such purely academic question, say, what is a further evolution of the decrease of the energy and entropy carried by the accreting mass that was swallowed by the $\mathrm{BH}$; or what is further evolution of the coalescence and merger of binary BHs at grazing collision of members, when triggered by the emission of gravitational waves their orbits will tighten by spiraling inwards. At this, immediately the question arises whether or not yet observationally unverifiable standard four laws of the mechanics for a stationary, asymptotically flat, black BH in four dimensions will be valid as well for non-stationary processes of $\mathrm{BH}$ formation and growth. To the best of our knowledge, the PMBH does not answer the questions invoked. 
Notwithstanding, much remarkably efforts have been made in understanding of BH physics, many important issues still remain unresolved and, thus, a situation is unclear, than described so far. Therefore the purpose of Section 7 is to examine the meaning of such statements and to assess briefly the validity of the growth and merging properties of BHs within the PMBHs. The first goal is to review briefly the necessary ideas behind the various specific constructions and suggestions on the conceptual problems of GR, the singularities and the thermodynamics of BHs in semiclassical and quantum physics. The second goal encompasses the many discoveries which unlocked the mysteries or exposed some of the illusions of the considered field. Without it we cannot show how the matters stand, we almost bound of necessity to enter upon it, if we would write of them at all.

In this respect, we should deliberately refrain from presumption of such exotic hypothetical behaviors, which seem nowhere near true if one applies the phenomenological model. But, how one can be sure that some hitherto unknown source of internal pressure does not become important above such extreme densities and halt the collapse? The failure of the PMBH does not necessarily imply a failure of the $\mathrm{BH}$ concept in general. In spite of a thorough search no reason could be found to introduce the required huge energy scale in $\mathrm{BH}$ physics but considerable change of properties of space-time continuum in density range far above nuclear density. We believe that a complete, self-consistent gravitation theory will smear out singularities at huge energies. This may shed further light upon the growth and merging phenomena of astrophysical BHs.

To fill the void which the standard PMBH presents and to innovate the solution to alluded problems, in what follows, we should recount some of the highlights behind of MTBH, whereas the infrastructures will inevitably be accommodated inside the EH. To start with, in Section 8 we discuss the proposed general theory of DSTC (Ter-Kazarian, 1986, 1989c, 1997, 2010, 2011, 2012, 2015), underlying the MTBH. This theory, in particular, involves a drastic revision of a role of local internal symmetries in physical concept of curved geometry, and explores the most important processes of spontaneous breaking of gravitation gauge symmetry and rearrangement of vacuum state. Using the language of fundamental geometric structure-distortion gauge induced fiber-bundle, it leads to modified gravitational theory as a corollary of the spacetime deformation/distortion framework. We generalize the standard gauge scheme via the concept of distortion gauge field which acts on the external spacetime groups, and that accounts for gravitation gauge group $G_{R}$ generated by hidden local internal symmetry implemented on the maximally symmetric (MS) - space. Thereby we construct a formalism of unitary mapping of the fields and their dynamics from the flat space to the curved space, and vice versa. In the framework of method of phenomenological Lagrangians, we relate the group $G_{R}$ to non-linear realization of the Lie group $G_{D}$ of distortion of local internal properties of the 12dimensional $M_{12}$ MS-space. We study the geometrical structure of the space of parameters in terms of Cartan's calculus of exterior forms introduced through the appropriate Maurer-Cartan's structure equations. The metric in our approach is no more a fundamental field. Hence, we extend the curvature of the spacetime continuum to general distortion as the theory of spontaneous breaking of distortion symmetry. The fundamental field is distortion gauge field and, thus, all the fundamental gravitational structures in fact - the metric as much as the coframes and connections - acquire a distortion-gauge induced theoretical interpretation. Particular attention is given to realization of the group $G_{R}$ by the hidden local internal symmetry of abelian group $U^{l o c}=U(1)_{Y} \times \operatorname{diag}[S U(2)]$ implemented on the $M_{12}$, where $Y$ is the hypercharge. We address the rearrangement of vacuum state in gravity resulting from these ideas. Spontaneous symmetry breaking is achieved by Higgs scalar. Two neutral gauge bosons were mixed to form the massless and massive components of distortion field. Hence, a massive distortion field component may cause an additional change of properties of the space-time continuum beyond gravity at huge energies. This theory is renormalizable, because gauge invariance gives conservation of charge, also ensures the cancelation of quantum corrections that would otherwise result in infinitely large amplitudes. Moreover, one of the underlying principles of MS-space interacting quantum field theory is that the vacuum is well-determined and unique, in particular, $\mid$ in $\rangle=\mid$ out $>$ (up to a phase factor).

The available solar system observational verifications offer many opportunities to improve tests of relativistic gravity. We rather have shown that the agreement is satisfactory between the proposed theory of gravity and mentioned observational verifications. Thereby the free adjustable parameter 
$\varepsilon$ in metric component, in case of static spherically symmetrical system, $g_{00} \simeq 1-\frac{R_{g}}{\tilde{r}}+\varepsilon \frac{R_{g}^{2}}{\widetilde{r}^{2}}$, can be written in terms of Eddington-Robertson expansion parameters $\beta$ and $\gamma$, as $\varepsilon=2(\beta-\gamma)$. The best fit for satisfactory agreement between the proposed theory of gravity and observation is reached at $\varepsilon=(2.95 \pm 3.24) \times 10^{-5}$. Moreover, it is consistent with GR up to the limit of neutron stars. However, this theory manifests its virtues applied to the physics at huge energies. Whereas a significant change of properties of space-time continuum, so-called inner distortion (ID), arises simultaneously with the strong gravity.

In Section 9 we discuss some basic knowledge and a few more technical details of MTBH (TerKazarian, 1989a,b, 1990, 1991, 1992, 2001, Ter-Kazarian \& Yerknapetian, 1995), which would certainly be helpful for the reader. The MTBH is a first-principles treatment of a fundamental superdense protomatter physics, but it also has an actual physical realization of Ambartsumian's fundamental vision. We necessarily proceed with some preliminaries on generic of the MTBH as a guiding principle to make the rest of paper understandable. Exploring the most important process of a spontaneous breaking of gravitation gauge symmetry at huge energies, MTBH is an extension of the PMBH to huge energies. This has smeared out a central singularity of a BH at very strong gravitational fields, replacing it by the equilibrium superdense proto-matter core (SPC), subject to certain rules. Encapsulated in an entire set of equations of equilibrium configuration, the SPC is a robust structure that has stood the tests of the most rigorous theoretical scrutinies of a stability (Ter-Kazarian et al., 2007). This approach manifests its practical and technical virtue in the most remarkable drawback of MTBH, which is the fact that, instead of infinite collapse and central singularity, an inevitable end product of the evolution of massive object is the stable SPC. This will ultimately circumvent a principle problem of an observer's inability to access the degrees of freedom that are hidden beyond the horizon, and a necessity to assign the elusive entropy to BH. This in somehow or other implies that a physical entropy is assigned to SPC as a measure of the large number of thermodynamical real microstates of proto-matter, which is compatible with a concept of ergodicity. Due to it, MTBH is proved to be quite fruitful for the study of outlined below fundamental non-stationary ultra-high energy phenomena. We describe finite though unbelievably extreme conditions held in SPC, where, nevertheless, static observers are existed. The stable equilibrium holds as well in outward layers and, thus, an accumulation of matter is now allowed around the stable SPC. An external physics of accretion onto the SPC in the earlier half of its lifetime is identical to the processes in Schwarzschild's model. A crucial difference in the model context between the phenomenological and microscopic models of black hole comes in when one looks for the spontaneous breaking of gravitation gauge symmetry at huge energies, and thereof making room for growth and merging behavior of black holes. It should be emphasized that the key to our construction procedure is widely based on the premises of our experience of accretion physics. Therefore, what we have presented here has all the vices and virtues of the classical scenario of runaway core collapse which has always been a matter of uncertainties and controversies. Nevertheless, we caution that these entire constructions will be valid as well in the case if some hitherto unknown yet mechanism in Nature will in somehow or other way produce the superdense proto-matter, away from the accretion physics.

With this perspective in sight, in Section 10, we have undertaken a large series of numerical simulations with the goal to trace an evolution of the mass assembly history of 377 plausible accreting supermassive black hole seeds in AGNs to the present time and examine the observable signatures today (Ter-Kazarian, 2014, 2015, Ter-Kazarian et al., 2007). Given the redshifts, masses and luminosities of these black holes at present time collected from the literature, we compute the initial redshifts and masses of the corresponding seed black holes. For the present masses $M_{B H} / M_{\odot} \simeq 1.1 \times 10^{6}$ to $1.3 \times 10^{10}$ of 377 black holes, the computed intermediate seed masses are ranging from: $M_{B H}^{\text {Seed }} / M_{\odot} \simeq 26.4$ to $2.9 \times 10^{5}$. We compute the fluxes of UHE neutrinos (Ter-Kazarian, 2001, 2014, 2015, Ter-Kazarian et al., 2007). The AGNs are favored as promising pure UHE neutrino sources, because the computed neutrino fluxes are highly beamed along the plane of accretion disk, peaked at high energies and collimated in smaller opening angle $(\theta \ll 1)$. While hard to detect, the extragalactic ZeV-neutrinos may reveal clues on the puzzle of the origin of UHE-cosmic-rays with energies exceeding $1.0 \times 10^{20} \mathrm{eV}$, as they have the advantage of representing unique fingerprints of hadron interactions and, therefore, can initiate the cascades of UHE-particles. We also study neutrino 
cooling and fueling at disk accretion onto the SPC in AGN, and origin of UHE G.R.s (Ter-Kazarian, 2001, Ter-Kazarian et al., 2003).

In Section 11, we discuss the most striking recent revolutionary NuSTAR discovery of the first rare and mighty ultraluminous X-ray pulsations (Ter-Kazarian, 2016a,b). The pulsed luminosity of M82X2 is the most extreme violation of the Eddington limit and could be reconciled with that in the model of magnetic neutron star pulsar only by very arbitrary assumptions on geometric beaming of accretion flow on neutron star. Instead of making such assumptions, we tackle the problem by the implications of MTBH, without the need for significant breaking of Eddington limit. The M82X-2 is assumed to be a spinning intermediate mass black hole resided in final stage of growth. As a corollary, the thermal blackbody X-ray emission, arisen due to the rotational kinetic energy of black hole, escapes from event horizon through the vista to outside world which is detected as ultraluminous X-ray pulsations. The M82X-2 indeed releases $\simeq 99.6$ of its pulsed radiative energy predominantly in the X-ray bandpass $0.3-30 \mathrm{keV}$. We produce a coherent picture of the pulsed radiation of M82X-2: we derive a pulse profile and give a quantitative account of energetics and orbital parameters of the semi-detached X-ray binary containing a primary accretor M82X-2 of inferred mass $M \simeq 138.5-226 M_{\odot}$ and secondary massive, $M>48.3-64.9 M_{\odot}$, O/B-type donor star with radius of $R>22.1-25.7 R_{\odot}$, respectively. We compute the torque added to M82X-2 per unit mass of accreted matter which yields the measured spin-up rate.

However, we need to be more rigorous about a geometry which describes rotating axisymmetric black holes. Therefore, in Section 12 we analytically study the microscopic model of stationary and axisymmetric rotating black hole (Ter-Kazarian, 2016a). There are deep conceptual and technical problems involved, and these provide scope for the arguments discussed, which are carefully presented in both mathematical and physical terms. We derive field equations and obtain both internal and global vacuum spacetime solutions. The most remarkable feature of microscopic model of a rotating black hole is that, in earlier part of its lifetime, the external physics outside of outer oblate event horizon of accretion onto a black hole is identical to the processes in Kerr's model. But, there is also a crucial difference between internal physics of Kerr and microscopic rotating black hole models, that is, a central ring singularity of the Kerr black hole cannot occur, which is now replaced by finite though unbelievably extreme conditions held in the central part of rotating SPC, where the static observers exist. The corrections introduced by this theory to the characteristic phase profile of M82X-2 of previous model are calculated (Ter-Kazarian, 2016a, Ter-Kazarian \& Shidhani, 2017).

In Section 13 we further expose the assertions made in MTBH, for the physics of intermediate mass black holes (IMBHs) (Ter-Kazarian \& Shidhani, 2019). This allows to construct microscopic models of accreting IMBHs. The mass estimates collected from the literature of all the observational evidence for $137 \mathrm{IMBH}$-candidates, even though there are still large uncertainties, allow us to undertake a large series of numerical simulations to derive all their essential physical characteristics. We compute among the others the masses, redshifts and growth-time scales of seed BHs. The luminosities of IMBH-candidates are computed by using a derived new scaling mass-luminosity relation.

The physical outlook and concluding remarks are given in Section 14.

\section{Ambartsumian's cosmogony}

Prior to Ambartsumian's work, the astronomers were applying the kinematic theory of gases to stars. In reality, the stars interact in accordance with Newton's law and special physical statistics should be used for investigations of stellar systems. Ambartsumian laid the foundations of such statistics and applied it to multiple stars and star clusters. This becomes further the basis for the statistical mechanics of the stellar systems. He determined the distribution of elements of binary star orbits at equilibrium and suggested that such state has not yet arrived. He also studied the process of star cluster disintegration due to escape of high-velocity stars and discovered that the process did not yet go too far. This led to a conclusion that the age of the galaxies is less than $10^{10}$ years. It was assumed previously that the stars were much older. Ambartsumian clearly proved the short scale of galactic life in a dispute (1935-1937) with the known British astronomer Sir James Jeans. The main feature of proposed earlier models describing the origin and evolution of stars and galaxies is the 
initial hypothetical state of tenuous gas, while stars originate through the condensation of this gas. The stellar matter remains a classical perfect gas throughout all of initial phases of evolution.

However, based on the observations in 1947 of stars of $\mathrm{O}$ and $\mathrm{B}$ spectral types and T Tauri and flare stars that cluster very loosely, he was first to suggest a concept of OB and T associations, coined by him as Stellar Associations (Ambartsumian, 1947, 1949, 1954, 1955, 1958c, 1960, 1971, 1984, 1985, ?). The observations indicate that in real stellar systems of the trapezium type one of the components belongs to $\mathrm{O}$ or B spectral types. Such stars are of recent formation and the number of revolutions they complete in the system is expected to be small. However, the observations show that a few multiple stars of later spectral classes also possess trapezium-like configurations. Of course, the configurations we observe on the sky are projections of true space configurations. Therefore, even if there are no real trapezium configurations of later- type stars, when they are projected on the sky a small percentage (8\%) of apparent configurations of the trapezium type will appear. This is almost precisely the percentage of trapezium configurations observed in cases where the components of multiple stars do not belong to the $\mathrm{O}$ and $\mathrm{B}$ spectral types. In other words, there are no or almost no real configurations of the trapezium type among the late-type multiple stars. The analysis of observational data of young stars leads to the conclusion that very dense protostellar bodies give birth to more or less numerous groups of stars. These considerations prove that the ideas about the simultaneous emergence of stars and diffuse matter from denser and more massive bodies are more fruitful and correspond to reality. In these associations the stars differ from the surrounding stars by having a greater partial density (i.e., density of stars of a given spectral class).

On these premises, Ambartsumian explained relatively rarely observed forms of activity of a nearby red dwarf stars, which have evolutionary significance Ambartsumian (1971). These, first of all, are the transitions from a state of low luminosity to a state of higher luminosity, maintaining this for a long time. This was observed in the objects of FU Orion type (Fuors) and objects of Herbig-Haro. Fuors are the variable stars that can undergo unpredictable dramatic increases in brightness in the observed part by more than a hundred times in a short period of time, after which they retain increased luminosity for many years. Much attention is paid to the variable dwarfs among which the atmospheres of UV Ceti stars and $\mathrm{T}$ Tauri stars should be more attractive ones. In their spectra, bright lines and the emission of a continuous spectrum are observed, which are sometimes so strong that all absorption lines are veiled. The stars of type $\mathrm{T}$ Tauri are interesting as they meet in groups, forming associations. In 1953, Ambartsumian paid attention to the fact that in short burst periods, UV Ceti type variable stars acquire some features similar to characteristics of $\mathrm{T}$ Tauri stars, and that the T Tauri type stars along with continuous and irregular changes of brightness also show flare type changes. He concluded that these two types of objects are genetically related. The ultraviolet excess observed in some $\mathrm{T}$ Tauri stars has approximately the same energy distribution as the emission from flares of UV Ceti type stars. A comprehensive study of this phenomenon allows Ambartsumian in 1968 to conjecture that the flare stars are the earliest evolutionary stages of the dwarf stars. Moreover, he believes that the stage of a flare star follows a stage of $\mathrm{T}$ Tauri type at overlapping of these stages.

The nature of the source of non-thermal and corpuscular radiation of the prefuor is unknown. Ambartsumian argued that a superdense companion in the close neighborhood of that star (red dwarf) gives the thermal radiation of the prefuor. While it cannot be ruled out that the envelope is ejected by a super-dense companion. Proceeding from the fact that prefuors and flare stars are members of the same stellar associations, Ambartsumian concluded (Ambartsumian, 1971) that the processes of decay and release of energy in both cases have the same physical nature. Ambartsumian believes that each outburst is the result of the explosion (when brightness increase time is often measured in seconds) of a certain portion of the prestellar superdense matter, which came out from the inner layers of the star, where a prestellar matter remained for a relatively long time before explosion. A release of energy during decay processes of prestellar matter is reminiscent of the phenomena of radioactive decays. Ambartsumian, therefore, rejected the assumption of thermonuclear reactions as the main sources of this energy. Based on the idea that a portion of superdense prestellar matter transfers from the stellar interiors to the surface layers of young stars, Ambartsumian predicted the existence of fast and slow flares, which were later discovered, and gave an explanation to the Fuor phenomenon.

Ambartsumian predicted (Ambartsumian, 1949) the expansion of the associations, which was later 
observed. A mere comparison of statistical data concerning clusters and associations revealed that much greater number of systems are originated with positive, than with negative energies. The associations with with positive energies are very unstable and should disintegrate rapidly. From the fact that they did not disintegrate until now, it was concluded that they are not more than few million years old. This conclusion about the youth of stars contained in associations is supported by a number of other features of associations. He thus concluded that stellar births of explosive events are ongoing in the galaxies where stars are born in groups. Ambartsumian's stemming idea of stellar association as a dynamical entity of groups of stars with positive total energy had far-reaching implications for subsequent star formation theories. These ideas have a subsequent influence in his interpretation of active galaxies.

Ambartsumian was first to show in the early 1950's that the so-called radiogalaxies are not a result of accidental collision of two gigantic star systems, but represent a definite stage of internal evolution of galaxies of very high luminosity. Activity of galaxies, which manifests in their radio emission, is intimately connected with new formations, such as jet ejections extending from the galactic nucleus, spiral arms, and new star systems emerging within old galaxy. Ambartsumian was also first to call attention to the instability and activity of galactic cores and, thus, raised the issue of Activity of Nuclei of Galaxies (Ambartsumian, 1958a,b, 1961, 1962, 1965, 1966, 1968a,b). In his report at the Solvay Conference on Physics (Brussels 1958), Armbartsumian claimed that the activity of the galactic nuclei determines the most significant processes in the evolution of galaxies. He argued that so-called $D$ bodies exist in nature, which are responsible for the activity of the nuclei of galaxies, and that galaxies are born from the explosion of such D-bodies. Indeed, the correctness of Ambartsumian's general conception was incorporated with the subsequent discovery of quasi-stellar radio sources (quasars), which are starlike objects of exceptionally high luminosity at cosmological distances. These objects exhibit extraordinary violent processes including very fast fluctuation of luminosity, jet ejection, and high motion velocities of luminescent gases. There is very likely a close connection between galactic nuclei and quasars, and thus formation of developed structure of galaxies is, apparently, connected with their nuclei (or, possibly, with quasars). The nuclei of galaxies are the places where new objects are generated, and the fact that the activity can be found in galaxies with a wide range of distances strongly suggests that the activity goes on at all epochs. Violent events in galaxies are manifestations of mass creation. From these events huge fluxes of relativistic particles and rapidly moving gas clouds are generated. Quasars with intrinsic redshifts are also ejected, and they are probably related to the mechanism through which new galaxies are formed. Following Armbartsumian, it is reasonable to consider the possibility that systems of galaxies with positive total energy also originate in this way. He didn't believe that all cases groups and clusters of galaxies are stable and bound. Note that a common point of view has been that in general such systems are bound by unseen matter. In some cases this is clearly true, but it is not a general rule. Thus, a large part of the argument for the presence of much dark matter goes away, since it is mostly based on the use of the virial theorem. As well as when the interacting systems of galaxies are detected there is no good reason to believe that they are merging. This is what all of the observers like to assume, but from an observational point of view they may well be coming apart. Presumably the prejudice towards merging is based on the idea that gravity is the only force acting. But if explosive events are clearly seen, even if we do not understand them, the possibility that objects are separating rather than merging must be considered. Only in the cases in which tidal effects can clearly be seen to be present, as in the classical case of NGC 4038-39, is it reasonable to accept the merging hypothesis. There is much direct evidence that coherent objects - galaxies, quasars, etc. do not originate from initial density fluctuations in the Universe, but are generated and ejected from galactic nuclei as was originally proposed by Ambartsumian. The rapid release of large amounts of energy from galactic nuclei arise from creation processes, which taking place here and now in the present epoch. Thus he concluded that there exist clusters which are in a particularly active phase of evolution when new galaxies originate within them. Ambartsumian believed, see e.g. (Ambartsumian, 1961), that a strong argument in support of this view is provided by the two galaxies (NGC 3651 and IC 1182) out of the nuclei of which jets are ejected containing blue condensations. These galaxies with blue jets are also among the brightest members in the corresponding clusters. Finally, there are cases when blue components occur in the vicinity of other 
Ambartsumian's vision and further insight to key puzzles of ultra-high energy astrophysics

giant elliptical galaxies, which evidently represent a later stage in the evolution of the above blue condensations. The famous interacting galaxy pair NGC 3561 A\&B sometimes described as a spiral and elliptical galaxy in collision with a tidally bound dwarf galaxy created in in the collision. The embedded nebulosity is attributed to tidal effects. Several other active galaxies have been found to exhibit the same effect. Ambartsumian pointed out that this system may equally well be the result of the ejection of one galaxy from another, along the lines of M87 and M84 with this debris extending over $\sim 100 k p c$ with blue dwarf galaxies and starforming regions emerging out of the debris. Thus, he believed that this is an excellent example of one galaxy ejecting another, and the existence of much star formation and the high-redshift quasars shows that the creation process is at work. His view was that the galaxies which are expanding away from parent galaxy have been generated by processes in the nucleous of that galaxy. He gave as another example some of the small groups of galaxies and highly irregular clusters like the Hercules cluster, in which the kinetic energy of the visible galaxies is much greater than the potential energy. If this is so, Ambartsumian concluded that the phenomena all pointed to ejection from superdense state, and that the galactic nuclei must contain superdense bodies of huge mass and some kind of non-stellar objects of unknown nature. He, in fact, conjectured that such an eruptive activity is due to the violent outburst events of transformations of superdense matter in supermassive compact D-bodies in galaxies, away from the accretion physics, where the creation process is at work. This signifies that every nucleus is made up of three components: stellar population, gas and supermassive body. Dynamically, the nucleus evolves independently of the rest of the galaxy.

In all these ideas Ambartsumian was initially alone. For many researchers it is still not easy to admit the possibility of changing the traditional paradighm of the models of gravitational collapse, even that they leave an inexplicable gap in explaining how the stars and galaxies come into being. From their view point, the apparent difficulty with Ambartsumian's hypothesis (Ambartsumian, 1961) is coming from dynamical arguments that the time scale for such systems to disintegrate is $\leq 10^{9}$ years, a value much less than $H_{0}^{-1}$, and much less than the time scale associated with galaxy formation and evolution in the big-bang cosmology. For example, Ambartsumian's estimate of age of M 82 employed only the fact that it has velocity higher than the velocity of escape from the M 81 group. All the members of the group M 81- M 82 are well known and therefore the upper limit of luminous mass can be estimated for certain, while any estimate of non-luminous mass will be quite arbitrary. So he was concluded that M 81 may be much older than M 82 and only M 82 (and perhaps NGC 3077 ) is of recent origin. But, of course, one also may consider the possibility that in this system of galaxies there exists rather more mass than is indicated by the luminous galaxies. However, the pressure of the further observations does its work, slowly but steadily. Generally though, they produce almost innumerable evidence in favor of ejections and explosions and are rather scanty regarding the processes of condensation and collapse. The concept of AGNs was widely accepted a few years later. A thousands of galaxies with strong ultraviolet excess have been discovered ever since in Byurakan Observatory under his direction. Ambartsumian's student, B. Markarian (well known for Markarian galaxies), completed a brilliant survey of galaxies known by his name with UV excess using the 1-m Schmidt telescope of the Byurakan Observatory. A study of the structure of the irregular clusters of galaxies leads one to the conclusion that often they are made up of several superimposed groupings. An interesting example of such a grouping was pointed out by Markarian: the chain of bright galaxies in the Virgo cluster containing NGC 4374, 4406, 4438 and others. This wonderful arc of eight bright galaxies is presumed to represent a physical grouping within the Virgo cluster. On the other hand facts about the radial velocities of the members of this group undoubtedly establish its positive total energy. Such facts are pronouncing an indictment against the ideas supporting the condensation processes. Thus, his conclusion was that observations produce almost innumerable evidence in favor of ejections and explosions and are rather scanty regarding the processes of condensation and collapse. In the observable Universe the processes of expansion and diffusion are responsible for the majority of changes occur now. These violent explosions were the manifestations of mass creation events. 


\section{The degenerate superdense gas of elementary particles}

Following Ambarstumian, three series of observational data providing an evidence in favor of superdense initial state are: (i) the galaxies and spiral arms are formed from their nuclei (Ambartsumian, 1958a,b), which have small sizes and high density; (ii) close groups of stars and Trapezium-type systems in associations and, in particular, in central regions of large gaseous nebulae found in O-associations; the phenomenon of flares in UV Cetl stars, and in many of the T-association members (Ambartsumian, 1958c). The most important property of superdense states, as will be seen from the following, must be the presence of hyperons in the star, in addition to neutrons. Let consider the processes releasing energy during transition from metastable state to stable one. Since, at sufficiently low temperatures, the nucleon (neutron or proton) gas is highly degenerate, hyperons with energies lower than a threshold value will become stable, because in accordance to Pauli's exclusion principle, the nucleons arising from their decay cannot find a place in phase space. For the same reason, the interconversion of different types of hyperons is also impossible. The hyperons present must also form a degenerate gas. Then, following Ambartsumian \& Saakyan (Ambartsumian \& Saakyan, 1960), we consider here a degenerate nucleon-electron gas with density of nuclear density, or higher, at a temperature $T=0$. At densities above certain value, the threshold Fermi energies for nucleons and electrons become so high that to maintain minimum total energy of the gas it is favorable if some of the nucleons should have changed into hyperons. Here the authors are not interested in the way in which superdense states are formed. To determine the relative abundance of the various types of particles as a function of density, they used a table of elementary processes of all standard leptonic transitions in which nucleons (n,p) converse into $\Lambda, \Sigma$ and subsequently $\Xi$-hyperons, to obtain respective relations between the chemical potentials $\mu_{i}$ of elementary particles $n, p, \Lambda, \Sigma, \Xi, e, \nu, \bar{\nu}$ as follows: $\mu_{\Xi^{0}}=\mu_{\Sigma^{0}}=\mu_{\Lambda}=\mu_{n}$, $\mu_{\Sigma^{+}}=\mu_{p}=\mu_{n}-\mu_{e}, \mu_{\Xi^{-}}=\mu_{\Sigma^{-}}=\mu_{n}+\mu_{e}$, which is valid for particles obeying Fermi statistics at $T=0$. The chemical potentials $\mu$ of the particles equal to their threshold Fermi energies. The $e, \nu, \bar{\nu}$ denote electron, neutrino and antineutrino, respectively. Also $\mu_{\nu}=\mu_{\bar{\nu}}=0$, because neutrinos and antineutrinos escape from the volume of the star as soon as they are generated without experiencing any interactions (the cross section for the interaction of neutrinos with electrons is of the order $10^{-44} \mathrm{~cm}^{2}$ ). A derivation of concentrations of various particles in a highly degenerate baryon gas at absolute zero temperature is based on the following supplementary assumptions: (i) At equilibrium, the energy of the system should be a minimum. (ii) Conservation of the number of baryons in all the processes leading to establishment of a state of statistical equilibrium of configuration. (iii) The star as a whole, as well as its separate macroscopic regions, should be electrically neutral. Consequently, the problem is reduced to determining the minimum of the energy density of the medium of possible various particles, with the supplementary conditions stemming from the above assumptions. The accepted procedure for this is to equate to zero the derivatives of the resulting extended energy function with respect to the particle concentrations, one finds the necessary conditions for the energy of mass distribution to be a minimum. The final problem is now to find the variation of the particle concentrations as the total number of baryons $N$ increases from zero to very high values. There will be a number of phase changes as $N$ increases. However, to find the values of density attained in any given cosmic body, which is in equilibrium state under the action of its own gravitational forces, one should employ GR to derive the equation of state $\rho=\rho(P)$, or in parametric form. This reveals the overall scenario. At density $\rho<\rho_{n}=1.28 \times 10^{7} \mathrm{gcm}^{-3}$, the gas consists of protons and electrons. At $\rho=\rho_{n}$ neutrons appear. At density $\rho>10^{8} \mathrm{gcm}^{-3}$, the number of neutron is much larger that numbers of protons and electrons. The individual atomic nuclei will no longer play an important role. At baryon density $N \geq 6.4 \times 10^{38} \mathrm{~cm}^{-3}$, i.e. $\rho=\rho_{\Sigma^{-}}=1.1 \times 10^{15} \mathrm{gcm}^{-3}$, the first hyperons appear. Although the rest masses of the hyperons $\Lambda, \Sigma^{+}, \Sigma^{0}$ are smaller than that of the $\Sigma^{-}$, nevertheless first $\Sigma^{-}$-hyperons appear. Highly degenerate matter contains hyperons and $\mu^{-}$-mesons in addition to nucleons. At $\rho=\rho_{\Lambda}, \Lambda$-hyperrons appear, and with a further increase of density, other heavier hyperons make an appearance altogether with . For baryons densities exceeding $5 \times 10^{16} \mathrm{gcm}^{-3}$, the following difficulties arise in the study of state equation: (i) very strong repulsive forces arise whose properties are not know well; (ii) the relative concentrations of various types of baryons can be strongly affected by the presence of higher hyperons having masses grater than that of the $\Xi$-hyperon. At still higher densities, $\pi$-mesons must also make their appearance as a Bose gas. 


\section{On equilibrium configurations of superdense degenerate gas masses}

Continuing along this line, Ambartsumian \& Saakyan (Ambartsumian \& Saakyan, 1961a) seek to find the solution to Einstein's equations for spherically symmetric superdence configurations. Oppenheimer \& Volkoff (Oppenheimer \& Volkoff, 1939) have shown that the solution of Einstein's equations in the static case is reduced to solution of comparatively simple differential equations. The Oppenheimer \& Volkoff's equations can be readily integrated for the case of configuration of ideal gas. Proceeding with the initial conditions $\rho=\rho(0)$ and $P(0)=P(\rho(0))$, where $\rho(0)$ is the central density of the given configuration, the integration is carried out step by step all the way up to boundary $R$, where $\rho=\rho(R)=P(R)=0$. In this case, the numerical integration of the equations does not encumbered for the finite central densities. The calculations shown that the masses of equilibrium configurations of a non-rotating baryon ideal gas are of the ordder of a half of a solar mass, and that the radii reach out several kilometers. It would be of interest, however, to consider also the limiting case of $\rho(0) \rightarrow \infty$ when baryon gas becomes extremely relativistic $P \approx \rho / 3$. At small interparticle distances $\sim 0.4 \mathrm{fm}$ or less, strongly intense repulsive forces apparently come into action. The baryonic gas, therefore, can no longer be considered an ideal gas at baryon densities exceeding $10^{40} \mathrm{~cm}^{-3} N$ which corresponds to the short distances $l<0.5 \mathrm{fm}$. It is possible roughly take into account the potential $U(N)$ of interactions in state equation. The masses of the degenerate configurations calculated for a real Fermi gas of baryons under the assumption that repulsive forces are active between the baryons, are appreciably larger than the masses of configurations of an ideal gas. However, small masses of the order of a solar mass are also obtained in this case. Even if the law of repulsion should be altered, which assumed to prevail, this still be unable to leads to the masses far exceeding a solar mass. The sizes and masses of the outer regions of a baryon star, i.e. neutron or proton-electron layers, comprise a small part of the total mass and size of the star at fairly high central densities. The bulk of the star's mass in those cases goes into the hyperon core. Such configurations are coined hyperon-configurations. Configurations with slightly lower central densities $\rho<10^{15} \mathrm{gcm}^{-3}$ lack hyperon cores and consists entirely of neutrons.

\section{Internal structure of hyperon configurations of stellar masses}

By numerical integration of equations determining the configuration of degenerate baryonic gas, all the necessary parameters specifying the internal structure of configurations of stellar masses are obtained in previous Section. Following Ambartsumian \& Saakyan (Ambartsumian \& Saakyan, 1961b), Fig. 1 gives in somewhat greater detail the plots of major characteristics of internal structure of configurations of ideal Fermi gas against the distance $\underline{r}$ from the center: (a) $t_{n}(\underline{r})$; $(b) U(\underline{r})$; metric components (c) $g_{r r}(\underline{r})$ and (d) $g_{00}(\underline{r})$, and (e) the dependence of mass of configuration on total number of baryons. Here and throughout we use following notational conventions: the particle concentrations are expressed in terms of parameter $t_{k}=4 \operatorname{arsh}\left(p_{k} / m_{k} c\right), m_{k}$ is the mass of $\mathrm{k}$-th particle, $p_{k}$ is the critical Fermi momentum $p_{k}=\left(6 \pi^{2} / a_{k}\right)^{1 / 3} h N_{k}^{1 / 3}$ for the k-th particle, $a_{k}=2 S_{k}+1$ is the number of particle spin states, $\underline{r}$ is the distance from the center, the function $U(\underline{r})$ gives approximate idea of the mass, in units of solar mass, concentrated in a sphere of radius $\underline{r}$. Inside the configuration volume, the value of time component $g_{00}$ of metric tensor achieved at accepting starting point that the matter exists in a state of thermodynamic and mechanical equilibrium. The numbers attached to the lines indicate the value of $t_{n}(0)$ for the given configuration. The corresponding characteristics of internal structure of configurations of real Fermi gas are not referred for obvious reason as the non-Euclidian nature of space in this case is more strongly pronounced in the volume of the configurations and adjacent regions. The study of the relationship between stellar parameters and total number of baryons in the star, and the dependence of that number on the density value at the center are problems of heightened interest. For example, plot (e) shows the dependence of mass of configuration on total number of baryons. Numbers next to circles indicate values of parameter $t_{n}$ at the center for those points. Solid line refers to real gas models, and dashed line refers to ideal gas models. The range $0.6<t_{n}(0) \lesssim 1.8$ is traced out in common by both curves. Models of an ideal gas corresponding to points on the lower branch of the graph are purely neutronic models. Points on the upper branch correspond to hyperon 


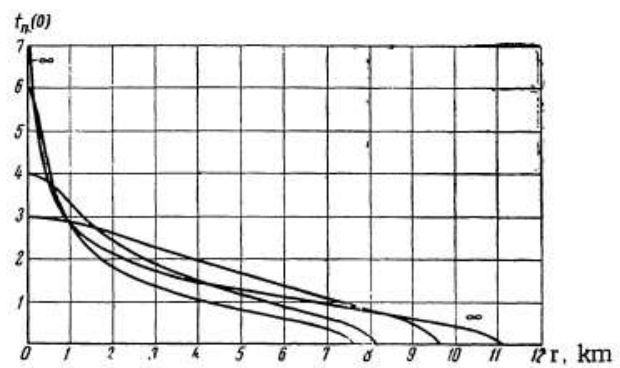

(a)

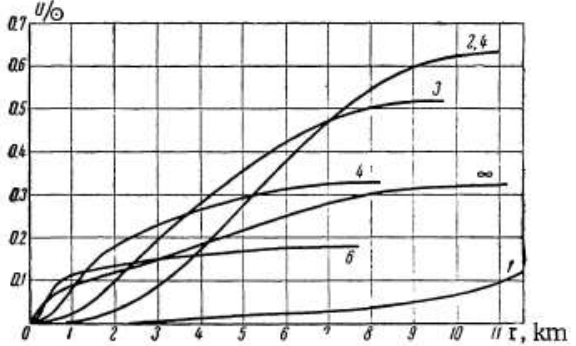

(b)

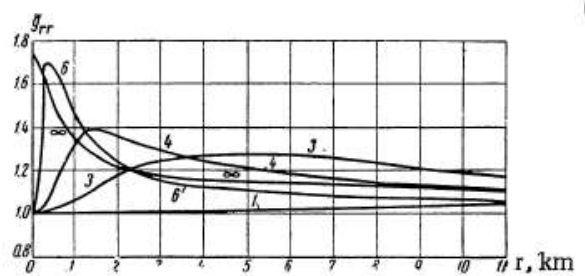

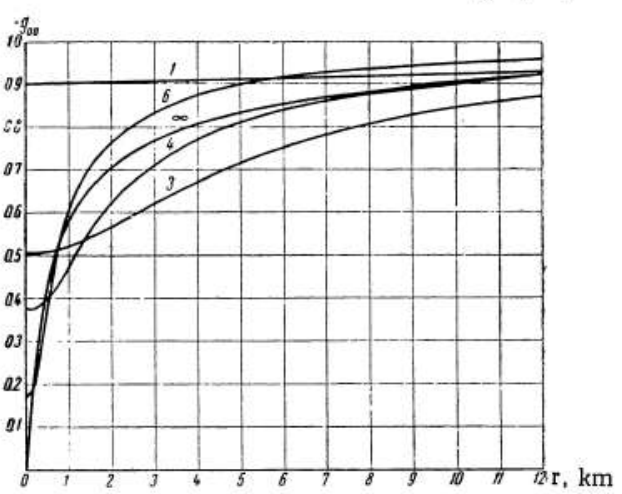

(d)

(c)

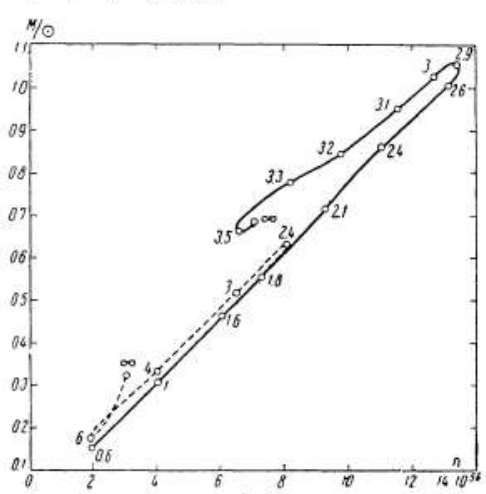

(e)

Figure 1. (a) The parameter $t_{n}(\underline{r})$ against the distance $\underline{r}$ from the center for four configurations corresponding to different central values of $t_{n}(0)$. (b) The function $U(\underline{r})$ which gives approximate idea of the mass, in units of solar mass, concentrated in a sphere of radius $\underline{r}$, for six different ideal gas states. The curves for $t_{n}(0)=1$ extends out to $\underline{r}=21.1 \mathrm{~km}$. (c) Dependence of metric component $g_{r r}$ on $\underline{r}$ for five different ideal gas states. As $\underline{r} \rightarrow \infty$, the function tends to its Euclidian value of unity. (d) Dependence of time component $g_{00}$ of metric tensor on $\underline{r}$. The $g_{00}=1$ corresponds to Euclidian value of unity. Distances are given in kilometers. (e) Dependence of mass of configuration on total number of baryons. Numbers next to circles indicate values of parameter $t_{n}$ at the center for those points. Solid line refers to real gas models, and dashed line refers to ideal gas models. The range $0.6<t_{n}(0) \lesssim 1.8$ is traced out in common by both curves.

configurations. In the case of real gas, hyperon configurations are found on both branches, but states corresponding to upper branch points contain still higher percentage of hyperons. Starting from some value $\underline{n}$ of number of baryons in the star (equal to $6.5 \times 10^{56}$ for real gas) there correspond to each $\underline{n}$ two or three equilibrium configurations. It is obvious that the configuration of lesser mass will be the more stable. A large mass exhibits a high density at the center. This must signify a large gravitational mass defect, in consequence of which the total mass of the configuration should be smaller in this case. A star located on the upper branch of the curve must undergo a transition to a state belonging to lower branch, in response to certain perturbations. Such transition will be accompanied by the release of tremendous amount of energy from the star, an amount of the order of $10 \%$ of the star's intrinsic energy. Such question merit special scrutiny. The gravitational mass defect $\triangle M=n m_{H}-M$ of baryon stars has been investigated. Reviewing notations $M$ is the mass of the star, $n$ is the number of baryons present, and $m_{H}$ is the mass of hydrogen atom. It is shown that the configuration with $t_{n}(0) \lesssim 2.4$ is absolutely stable in the case of ideal gas, while the configuration with $t_{n}(0) \lesssim 2.9$ is absolutely stable in the case of real gas. The rest of configurations are either metastable or unstable. There is one intriguing feature in the unstable branches, namely that for $t_{n}(0) \gtrsim 4.2$ in the case of 
ideal gas and for $t_{n}(0) \gtrsim 3.15$ in the case of real gas, the binding energy $\triangle M$ becomes negative (see also sect.5). Such states will be less stable than the others.

\section{The mass defect of baryon stars}

In this Section we outline the key points of a more detailed analysis of the absolute gravitational mass defect, $\triangle M=n m_{n}-M l$, of neutron configurations (Ambartsumian \& Saakyan, 1961b, 1965). This is of particular interest to astrophysicists. Note that the value of the mass defect $\triangle M$ is to some degree dependent on the selection of the frame of reference and therefore is not an invariant characteristic of the star. Obviously, for ordinary celestial bodies in all case $\triangle M>0$. The computations of baryon configurations have demonstrated that when central densities rise above a certain value $\rho_{1}(0)$ the absolute mass defect changes sign - it becomes negative. The value of $\rho_{1}(0)$ depends on the form of the equation of state for the baryon gas used in the computations. In models with a real baryon gas $\rho_{1}(0)$ has a lesser value than in models with an ideal gas. This can be attributed to the fact that in the case of a real gas, at densities greater than nuclear, the nuclear forces of repulsion between baryons is played important role. Naturally, the forces of repulsion facilitate the appearance of the considered effect. It follows that the value of gravitational mass defects for models with a real gas are not entirely correct, because in this case they are determined not only by gravitation but also by the effect of nuclear forces (attraction and repulsion). In order to exclude the influence of nuclear forces on the absolute mass defect and investigate the phenomenon in purer form, one will be concerned only with models of superdense stars consisting of an ideal baryon gas. The presence of hyperons and interaction between baryons were disregarded, in order to avoid complications unrelated to the considered problem. Thereby, at first, one uses Einstein's gravitational theory as a point of departure. Next, neutron configurations were also computed for the case when Newton's gravitational law is used as the point of departure. In this case one knowingly admitted a certain inconsistency, extending the computations to include configurations consisting of a relativistic neutron gas. However, in this case one had a definite purpose in mind: comparison of the exact and approximate computations in order to clarify the role of relativism of the baryon gas and the curvature of space and thereby determine the cause of the anomaly in the absolute gravitational mass defect. We shall note only a few common and important aspects of numerical computations. a) Although the results of computations of the parameters of neutron stars on the basis of Newton's and Einstein's gravity theories reveal an appreciable quantitative difference, nevertheless, in some most important respects there is good qualitative agreement. For example, Newton's theory of the mass, radius, and number of baryons in a star gives the correct orders of magnitude. b) According to both theories, all the parameters of the configurations are single-valued functions of the central den- sity $N(0)$. The opposite assertion is, in general, untrue. In certain regions the same value of some of the star parameters, such as mass, correspond to two (or even more) values of the central density. c) In the case of Newtonian models, the absolute gravitational mass defect for all possible static configurations has a positive value. The packing coefficient is in all cases an increasing function of the central density, with the exception of the region of extraordinarily high central densities, where a conspicuous minimum is recorded. d) In the case of relativistic models, the gravitational packing factor $\triangle M / M_{0}=f(n)$ exhibits anomalous behavior. At first, it increases with increase in density, at $N(0)=3 \times 10^{39} \mathrm{~cm}^{-3}$ it attains a maximum and then begins to decrease. At $N(0)=1.12 \times 10^{40} \mathrm{~cm}^{-3}$ the packing factor becomes negative. At $N(0)=2 \times 10^{41} \mathrm{~cm}^{-3}$, there is a deep minimum, approximately equal to -0.1 ; then the packing factor, oscillating with a small and strongly damping amplitude and continuing to remain negative as $\rho(0) \rightarrow \infty$, tends to a limit of -0.069 . The authors, therefore, conclude that the mass defect anomaly was caused by a catastrophic deviation from additivity of the internal energy due to warping of the spatial metric in the corresponding baryon configurations. In these configurations and in the Newtonian approximation the deviation from additivity (here the kinetic energy is additive and the potential energy is not) is strong, but inadequate for a change in the sign of the mass defect. Obviously, the configurations corresponding to the lower branch of the $f(n)$ curve when $3<t_{n}(0)<4.67$ are unstable in relation to the transitions to the upper branch, where the mass defect exhibits normal behavior. However, configurations with $t_{n}(0)>4.67$, having a negative absolute mass defect, are unstable not 
only in relation to transition to the upper branch, but also in relation to decay into a diffuse state. Since the mass defect is several percent of the mass of the star itself, an improbably large energy will be released in these transitions. The energy associated with one gram of star matter is an order of magnitude greater than the corresponding energy released in thermonuclear reactions in the combustion of hydrogen. It is important to note that the binding energy of each particle in a star is negative, so that the particles cannot escape individually to infinity. The escape of a certain number of baryons from a star requires the addition of supplementary energy to the remaining configuration from the outside. For this reason it cannot occur spontaneously. This means that transition of the system to a more stable state can occur only under the influence of very great perturbations. In this case expansion will occur, accompanied by heating of the celestial body. The corresponding transition will have the character of a cosmic explosion. These arguments concerning the fate of baryon configurations with an anomalous absolute mass defect were first presented in (Ambartsumian \& Saakyan, 1961b). The configurations with an anomalous value of the absolute mass defect (we refer to the entire branch of the curve with $t_{n}(0)>3$ ) should be of some importance to Ambartsumian's cosmogony. In order to relate the above considerations on the behavior of baryon configurations with an anomalous value of the absolute mass defect to the concept mentioned, it is necessary to construct models of superdense prestellar bodies with masses of a much greater order of magnitude than the solar mass.

\section{Assessment of growth and merging properties of BHs within $\mathrm{PMBH}$}

Wheeler in 1967 coined a spacetime region, where the gravitational field is so strong that no information carrying objects and signals can escape it, by the term 'a black hole', although the possibility of the existence of such objects was discussed a long time before this. At the end of the eighteenth century Michell and Laplace independently came to the conclusion that if the mass of a star were large enough its gravity would not allow light to escape. Though this conclusion was based on the Newtonian theory the obtained result for the size of such 'dark stars' (the gravitational radius) coincides with the later prediction of Einstein's theory of gravity. A principle feature that makes GR distinctively different from other field theories is the occurrence of curvature singularities in spacetime. The singularities lead to regions of the universe that cannot be observed. This causes an observer's inability to access the degrees of freedom that are hidden beyond the horizon which, in turn, leads to thermodynamical behavior of BHs. The astrophysical significance of the issue, and the importance of considering the gravitational collapse of a matter cloud within the framework of the GR theory, with reasonable physical properties for the matter included, stems from the fact that GR predicts that a star more massive than about five to eight times the mass of the Sun, cannot stabilize to a neutron star final state at the end of its life cycle. It must collapse continually under the force of its own gravity on exhausting its internal nuclear fuel, and there are no known forces of nature that would halt such a collapse. GR predicts that such a star must then terminate into a spacetime singularity where densities and spacetime curvatures blow up and the physical conditions are extreme. The estimates on the mass limit for a star in order to collapse, of course, are indefinitely vary depending on different models for the star's interior and equation of state for matter at very high densities.

In this Section we would like briefly re-examine (critically) the past and present of the PMBHs, and attempt to chart the future of the subject. Within respect to standard models, a hard look at the PMBH physics reveals at least the following severe problems:

(i) The geometrical interpretation of gravitation, having arisen from the dual character of the metrical tensor in its metrical and gravitational aspects, is a noteworthy result of GR. Being among the most significant advances in solving the key problems of cosmology and astrophysics, the GR certainly can claim remarkable success in the experimental and observational tests too. Nevertheless such a distinction of the gravitational field among the fields yields the difficulties in the unified theories of all interactions of elementary particles, and in quantization of gravitation. Some conceptual problems of the energy-momentum conservation laws of gravitational interacting fields, the localization of energy of gravitation waves, the role of singularities, and also severe problems involved in quantum gravity are still plaguing GR. As a geometrized theory of gravitation, the GR clashes from the very outset with basic principles of the field theory. This rather stems from the fact that Riemannian geometry, in 
general, does not admit a group of isometries, i.e., Poincaré transformations no longer act as isometries and, for example, it is impossible to define energy-momentum as Noether local currents related to exact symmetries. This posed severe problems in a Riemannian space interacting quantum field theory. The major unsolved problem is the non-uniqueness of the physical vacuum and the associated Fock space. Actually, a peculiar shortcoming of the interacting quantum field theory in curved spacetime is in the following two key questions to be addressed yet: a) the absence of the definitive concept of space-like separated points, particularly, in the canonical approach, and the light-cone structure at each spacetime point; b) the separation of positive- and negative-frequencies for completeness of the Hilbert-space description. Due to this, a definition of positive frequency modes, therefore, cannot be unambiguously fixed in the past and future, which leads to $\mid$ in $>\neq \mid$ out $>$, because the state $\mid$ in $>$ is unstable against decay into many particle $\mid$ out $>$ states due to interaction processes allowed by lack of Poincaré invariance. A non-trivial Bogolubov transformation between past and future positive frequency modes implies that particles are created from the vacuum and this is one of the reasons for $\mid$ in $>\neq \mid$ out $>$.

(ii) Conjectured in the framework of GR, the very source of gravitational field of a $\mathrm{BH}$ is a kind of meaningless curvature singularity at the central point of the stationary nonrotating Schwarzschild $\mathrm{BH}$, or a ring singularity at the center of the rotating axisymmetric Kerr BH, which are hidden behind the event horizon (EH). The theory breaks down inside the $\mathrm{EH}$ which is causally disconnected from the exterior world. The Kruskal manifold is the maximal analytic extension of the Schwarzschild and Kerr solutions inside EH, so no more regions can be found by analytic continuation. Either the Kruskal continuation of the Schwarzschild $(J=0, Q=0)$ metric, or the Kerr $(Q=0)$ metric, or the Reissner-Nordstrom $(J=0)$ metric, show that the static observers fail to exist inside the horizon. Any object that collapses to form a black hole will go on to collapse to a singularity inside the black hole. Any timelike worldline must strike the central singularity which wholly absorbs the infalling matter. Therefore, the ultimate fate of collapsing matter once it has crossed the black hole surface is unknown. The interior solution is not physically meaningful and essentially irrelevant. Black holes then present a major challenge that they render time reversibility impossible. Objects thrown into a $\mathrm{BH}$ can never be retrieved, because any timelike worldline must strike the central singularity which wholly absorbs the infalling matter. Any object that collapses to form a $\mathrm{BH}$ will go on to infinite collapse to a singularity inside the $\mathrm{BH}$. The $\mathrm{PMBH}$, therefore, ultimately precludes any accumulation of matter inside $\mathrm{EH}$ and, thus, neither the growth of $\mathrm{BHs}$ nor the increase of their mass-energy density could occur at accretion of outside matter, or by means of merger processes.

(iii) One of the most important open issues in the theory and astrophysical applications of modern day BH and gravitation physics is that of the Roger Penrose's Cosmic Censorship Conjecture (CCC). The CCC assumption that any physically realistic gravitational processes must not lead to the formation of a singularity which is not covered by an horizon, thus hiding it from external observers in the universe. This of course includes the complete gravitational collapse of a massive star which, if the $\mathrm{CCC}$ is true, must terminate generically into a $\mathrm{BH}$ final state only. Such a singularity is then crucial and is at the basis of much of the modern theory and astrophysical applications of BHs today. Despite the past four decades of serious efforts, we do not have as yet available any proof or even any mathematically precise formulation of the cosmic censorship hypothesis. The consideration of dynamical evolution of collapse is a crucial element of the CCC. Many solutions of Einstein field equations are known which present naked singularities (such as, for example, the super-spinning Kerr solutions), nevertheless almost none of these solutions can be obtained as the dynamically evolved final state of some initially regular matter configuration. For this reason, over the last decades a great deal of work has been done to test the CCC in the few dynamically evolving spacetimes we know. These are typically the scenarios that describe gravitational collapse in spherical symmetry, and some non-spherical collapse models have also been considered, for examples of critical collapse with angular momentum. In recent years, a wide variety of gravitational collapse models have been discovered where exact analytical calculations (e.g. (Joshi \& Malafarina, 2013) and references therein) have meanwhile shown that mass concentrations collapsing under their own weight will no longer form BHs as collapse endstate, rather naked singularities, except for configurations of highest symmetry which are, however, of measure zero among all initial data. By this, even the theoretical existence of 
$\mathrm{BHs}$ is no longer justified. The first examples were restricted to some classes of inhomogeneous dust collapse, and they were extended to the case of collapse in the presence of only tangential pressures, and perfect fluids. The existence of classes of pressure perturbations is shown explicitly, which has the property such that an injection of a small positive (or negative) pressure in the Oppenheimer, Snyder and Datt (OSD) model, or in a Tolman-Bondi-Lemaitre (TBL) inhomogeneous dust collapse to a $\mathrm{BH}$ (simplest generalization of the OS model), leads the collapse to form a naked singularity, rather than a BH (Joshi \& Malafarina, 2013). The classic OSD scenario is the basic paradigm for BH physics today, and the TBL models describe the most general family of dust, i.e. pressureless, collapse solutions. This result is therefore intriguing, because it shows that arbitrarily close to the dust BH model, we have collapse evolutions with non-zero pressures that go to a naked singularity final state, thus proving a certain 'instability' of the OSD BH formation picture against the introduction of small pressure perturbations. In such a case, the super-ultra-dense regions, or the spacetime singularity, that forms at the end of collapse would be visible to faraway observers in the universe, rather than being hidden in a $\mathrm{BH}$. Thus, rigorous calculations have shown that the expectations of the 1970s have been hasty, that CCC assumption has been premature, because while the CCC states that the OSD collapse final fate is necessarily replicated for any realistic stellar collapse in nature, the result here shows that an arbitrarily small pressure perturbation of the OSD model can change the final outcome of collapse to a naked singularity and therefore the OSD BH may be considered 'unstable' in this sense.

(iv) If the $\mathrm{BH}$ was being off the ordinary mass shell and carried no entropy, it would be possible to violate the cherished law of energy conservation and $2^{\text {nd }}$ law of thermodynamics, because the energy and entropy in the exterior spacetime could be decreased by throwing matter into a $\mathrm{BH}$. In the framework of incomplete theory, therefore, there is nothing left but to admit stepwise, without any substantiation, that the $\mathrm{BH}$ resides on the ordinary mass shell and it has entropy. That is, the law of increase of area (Hawking's area theorem) looks like the $2^{\text {nd }}$ law of thermodynamics for the increase of entropy, if one assigns an entropy to $\mathrm{BH}$ that is proportional to its surface, and that the surface gravity stands for a temperature. Moreover, the GSL was needed to present the second law of thermodynamics as valid. This is because the $2^{\text {nd }}$ law of thermodynamics, as a result of the disappearance of entropy near the exterior of $\mathrm{BHs}$, is not useful. Then the increase of these quantities may compensate the decrease of the energy and entropy carried by the mass that was swallowed. At first sight this definition of the laws of gravitation, and thereof for that of thermodynamics and entropy of BHs, seems quite natural, however, at closer inspection one finds that these intriguing ideas have encountered to the following severe objections:

a) The two features violate Hawking's area theorem - (1) in pair creation effectively a spacelike energy flux is involved - in contrast to the one of the essential postulates of the area theorem which requires that the energy-momentum tensor $T_{\mu \nu}$ should satisfy the dominant energy condition. This held if for all future-directed timelike vector fields $v$, the vector field $j(v) \equiv-v^{\mu} T_{\mu}{ }^{\nu} \partial_{\nu}$ is future-directed non-spacelike, or zero, i.e. no spacelike energy fluxes are allowed; (2) the mass of BH decreases during evaporation by energy conservation, as well as inevitably do the surface area and entropy.

b) The entropy of a thermodynamic system is a measure of the large number of the real physical microstates that an observer would not be aware of when measuring macroscopic parameters, and so-called no hair theorems allow $\mathrm{BH}$, in best case, to have only a single microstate.

(v) Yet about 47 years after its conjecturing, solid physical information regarding the physical origin of $\mathrm{BH}$ entropy is still lacking, which arises several puzzling questions. For example, since there is no unique rigid notion of time translations in a classical GR-dynamics, the BH entropy at least appears to be incompatible with any notion of ergodicity. Or, the equation for Hawking's black body radiation temperature, $T_{H}=c^{3} \hbar\left(8 \pi k_{B} G_{N} M\right)^{-1}$, clearly shows that the more mass is radiated away from the black hole, the hotter this becomes. What then is the endpoint of $\mathrm{BH}$ evaporation? Moreover, the thermal properties of thermodynamic systems reflect the statistical mechanics of underlying microstates. Entropy is normally a measure of the degeneracy of microstates, $\Sigma$, in some underlying microscopic description of a physical system, determined by Boltzmann's formula $S=k_{B} \log \Sigma$. Since the Bekenstein-Hawking entropy of generalized second law (GSL) of BH thermodynamics, $S_{H} k_{B}^{-1}=4 \pi G_{N} M^{2}(c \hbar)^{-1}=A_{H}\left(2 l_{P l}\right)^{-2}$ of a $\mathrm{BH}$, where $A_{H}$ is the area of the horizon and 
$l_{P l}$ is the Planck length $l_{P l}=\sqrt{G_{N} \hbar / c^{3}} \approx 10^{-33} \mathrm{~cm}$, is naturally a huge number, how can one exhibit such a wealth of microstates?

(vi) Certain gravitational backgrounds gave rise to thermal radiation from the vacuum. This provides an alternate conceptual means for understanding the physics of cosmological pair production at a wide variety of cosmological event horizons in exotic spacetimes. However, all these processes for certain do not give physical insight regarding the nature of the microstates of a $B H$ and nor does it offer a substantiated reason for the $B H$ entropy $S_{B H}$. Moreover, in semi-classical analysis of the Hawking evaporation process, if the correlations between the inside and outside of the $\mathrm{BH}$ are not restored during the evaporation process, then by the time that the $\mathrm{BH}$ has evaporated completely, an initial pure state will have evolved to a mixed state, i.e., information will have been lost in the process of $\mathrm{BH}$ formation and evaporation - the BH information loss paradox. If information is lost into the $\mathrm{BH}$, which is ascribable to the propagation of the quantum correlations into the singularity within the $\mathrm{BH}$, this put quantum field theory in curved spacetime in conflict with a basic principle of quantum mechanics, because of incompatibility with the unitary time evolution of a state vector in a Hilbert space. This violates the causality and energy-momentum conservation laws. The following three postulates of $\mathrm{BH}$ complementarity are not consistent with one another for a sufficiently old $\mathrm{BH}$ : (1) Hawking radiation is in a pure state, (2) the information carried by the radiation is emitted from the region near the horizon, with low energy effective field theory valid beyond some microscopic distance from the horizon, and (3) the infalling observer encounters nothing unusual at the horizon. These seem to require novel dynamics that nevertheless cause notable violations of semiclassical physics at macroscopic distances from the horizon. That is, one must sacrifice some principle: the equivalence principle, low energy effective field theory, or the nonexistence of high-entropy remnants at the end of $\mathrm{BH}$ evaporation.

(vii) The BH solutions to Einstein's equations, implying an existence of the event horizons, are defined only in asymptotically flat space-times. The Universe, in fact, is not asymptotically flat. So the event horizons, to which the laws of BH thermodynamics are referred, do not exist. The Schwarzschild $\mathrm{BH}$, fixing its temperature at infinity, has negative heat capacity. Similarly, in an asymptotically antide-Sitter spacetime fixing the BH temperature via the normalization of the timelike Killing vector at infinity is not justified because there is no such physically distinguished Killing field. The BHS are localized objects, thus one must be able to describe their properties and dynamics even at the quasilocal level. These difficulties lead to the need of a quasi-local formulation of BH thermodynamics. The subject of the quasi-local formulations is to describe the properties and the evolution of the so-called trapping horizon, which is a quasi-locally defined notion, somewhat as an apparent horizon.

(viii) Although no results on $\mathrm{BH}$ thermodynamics have been subject to any experimental or observational tests, the attempts of theoretical interpretation of the $\mathrm{BH}$ thermodynamics provide a basis for further research and speculation on the nature of its quantum gravitational origin. In the entanglement entropy and thermal atmosphere approaches, the relevant degrees of freedom are those associated with the ordinary degrees of freedom of quantum fields outside of the BH. The string theory implies weak coupling states, so it is not clear what the degrees of freedom of these weak coupling states would correspond to in a low energy limit where these states may admit a BH interpretation. There is no indication in the calculations that these degrees of freedom responsible for BH entropy should be viewed as being localized near the BH horizon. It is far from clear as to whether one should think of these degrees of freedom as residing outside of the BH (e.g., in the thermal atmosphere), on the horizon (e.g., in Chern-Simons states), or inside the BH (e.g., in degrees of freedom associated with what classically corresponds to the singularity). Moreover, the entanglement mechanism is not specific to BHs but to any spacetime with a bifurcating Killing horizon.

(ix) The efforts to understand the mysterious statistical mechanical properties of BHs has led to many speculations about their quantum gravity origin. Though not yet fully understood in general, the holographic principle is central to theories like the AdS/CFT (anti-de Sitter spaces/conformal field theories) correspondence. The holographic scaling suggests that a $\mathrm{BH}$ has far fewer degrees of freedom than we might expect. For a comprehensive review of recent progresses on the holographic understandings of the entanglement entropy in the AdS/CFT correspondence, BH entropy and covariant formulation of holography. As notably pointed out by these authors, even after quite intense 
efforts in AdS/CFT for recent years, fundamental mechanism of the AdS/CFT correspondence still remains a mystery. In particular, one cannot answer which region of AdS is responsible to particular information in the dual CFT. There is also an essential discrepancy between the entanglement entropy and the $\mathrm{BH}$ entropy, that the entanglement entropy is proportional to the number of matter fields, while the BH entropy is not. The former includes ultraviolet divergences as opposed to the latter.

(x) Within string theory, there is a class of BHs where some problems can be conveniently addressed, the so-called extremal BHs, for which the mass is tuned, so that the tendency to gravitational collapse is precisely balanced by the electrostatic repulsion. Consequently, the temperature vanishes and the black hole behaves somehow in this limiting case as if it were an elementary particle. These results, however, rely heavily on supersymmetry and serious difficulties are met in attempts to extend them to non-supersymmetric BHs. Up to date no one was able to make a convincing calculation of $\mathrm{BH}$ entropy based on statistical mechanics, which associates entropy with a large number of microstates being compatible with a concept of ergodicity. In this regard, proving the GSL is generally valid would require using quantum-statistical mechanics, but this discipline does not exist. So, due to existing discrepancies and absence of distinctive observationally tested predictions, there is no convincing reason to rely on the string theory in its present form. This then ruptures the familiar BH entropy illusion which has insufficient dimensions.

Only a true internal solution was able to give a reliable information on the thermodynamic behavior and entropy of $\mathrm{BH}$ if, and only if, it is known deep within the BH. At this point we cut short our discussion, and refer the interested reader to (Ter-Kazarian, 2021a, Ter-Kazarian \& Shidhani, 2017) for more details.

\section{The theory of distortion of the space-time continuum}

A remarkable surge of activity of investigations towards an extension of GR has arisen recently. They are geometrically expressible in the language of the fundamental structure known as a fiber bundle. This provides an unified picture of gravity modified models based on several Lie groups. All the approaches have their own advantages, but in the same time they are subject to many uncertainties. Currently no single theory has been uniquely accepted as the convincing gauge theory of gravitation, which will be able successfully to address the aforementioned problems.

In this respect we advocate with alternative proposal of the new physical perception of spacetime geometry - the theory of distortion of space-time continuum (DSTC) (Ter-Kazarian, 1986, 1989c, 1997, 2010, 2011, 2012, 2015) at huge energies (respectively, at short distances $<0.4 \mathrm{fm}$ ). As a corollary, in particular, DSTC leads to modified gravitational theory. The proposed gravitational theory involves a drastic revision of a role of local internal symmetries in physical concept of curved geometry, and explores the most important processes of spontaneous breaking of gravitation gauge symmetry and rearrangement of vacuum state. Much use has been made in proposed gravitation theory of the language of fundamental geometric structure - a distortion gauge induced fiber-bundle (TerKazarian, 1986, 1989c, 1997, 2010, 2012), incorporating with the spacetime deformation/distortionframework, (Ter-Kazarian, 2011, 2015) and references therein.

\subsection{A first glance at spacetime deformations}

In the framework of spacetime deformation theory, we consider a smooth deformation map $\Omega$ : $M_{4} \rightarrow \widetilde{\mathcal{M}}_{4}$, written in the terms of the world-deformation tensor $\Omega$, the general, $\widetilde{\mathcal{M}}_{4}$, and the flat, $M_{4}$, smooth differential 4D-manifolds. A following notational conventions will be used throughout this paper. All magnitudes related to the space, $\widetilde{\mathcal{M}}_{4}$, will be denoted with an over ${ }^{\prime} \sim$ '. We use the Greek alphabet $(\mu, \nu, \rho, \ldots=0,1,2,3)$ to denote the holonomic world indices related to $\widetilde{\mathcal{M}}_{4}$, and the second half of Latin alphabet $(l, m, k, \ldots=0,1,2,3)$ to denote the world indices related to $M_{4}$. The tensor, $\Omega$, can be written in the form $\Omega=D \psi\left(\Omega_{l}^{m}=D_{\mu}^{m} \psi_{l}^{\mu}\right)$, provided with the invertible distortion matrix $D\left(D_{\mu}^{m}\right)$ and the tensor $\psi\left(\psi_{l}^{\mu} \equiv \partial_{l} \widetilde{x}^{\mu}\right.$ and $\left.\partial_{l}=\partial / \partial x^{l}\right)$. The principle foundation of a world-deformation tensor comprises the following two steps. 
First step: The basis vectors $e_{m}$ at given point $\left(p \in M_{4}\right)$ undergo the distortion transformations by means of $D: \widetilde{e}_{\mu}=D_{\mu}^{l} e_{l}$.

Second step: The diffeomorphism $\widetilde{x}^{\mu}(x): M_{4} \rightarrow \widetilde{M}_{4}$ is constructed by seeking a new holonomic coordinates $\widetilde{x}^{\mu}(x)$ as the solutions of the first-order partial differential equations: $\widetilde{e}_{\mu} \psi_{l}^{\mu}=\Omega_{l}^{m} e_{m}$, here the conditions of integrability, $\partial_{k} \psi_{l}^{\mu}=\partial_{l} \psi_{k}^{\mu}$, and non-degeneracy, $\|\psi\| \neq 0$, necessarily hold. Next we write the norm $d \widetilde{s} \equiv i \widetilde{d}$ of the infinitesimal displacement $d \widetilde{x}^{\mu}$ on the $\widetilde{\mathcal{M}}_{4}$ in terms of the spacetime structures of $M_{4}: i \widetilde{d}=\widetilde{e} \widetilde{\vartheta}=\widetilde{e}_{\mu} \otimes \widetilde{\vartheta}^{\mu}=\Omega_{l}^{m} e_{m} \otimes \vartheta^{l} \in \widetilde{\mathcal{M}}_{4}$. The deformation $\Omega: M_{4} \rightarrow \widetilde{\mathcal{M}}_{4}$ comprises the following two subsequent 4 D deformations $\stackrel{\circ}{\Omega}: M_{4} \rightarrow V_{4}$ and $\breve{\Omega}: V_{4} \rightarrow \widetilde{M}_{4}$, where $V_{4}$ is the semi-Riemannian space, $\stackrel{\circ}{\Omega}$ and $\breve{\Omega}$ are the corresponding world deformation tensors.

The interested reader is invited to consult the original papers (Ter-Kazarian, 2011, 2015) for hard glance of the theory of spacetime deformation.

Finally, to complete this theory we need to determine $\widetilde{D}$ and $\widetilde{\psi}$. Keeping in mind aforesaid, we develop on the alternative framework of the General Gauge Principle (GGP), which is the distortion gauge induced fiber-bundle formulation of gravitation. In this, we restrict ourself to consider only the simplest spacetime deformation map, $\widetilde{\Omega}: M_{4} \rightarrow V_{4}\left(\breve{\Omega}^{\mu}{ }_{\nu} \equiv \delta_{\nu}^{\mu}\right)$.

\subsection{GGP}

We generalize the standard gauge scheme via the concept of distortion gauge field which acts on the external spacetime groups. Given the principal fiber bundle $\widetilde{P}\left(V_{4}, G_{V} ; \widetilde{s}\right)$ with the structure group $G_{V}$, the local coordinates $\widetilde{p} \in \widetilde{P}$ are $\widetilde{p}=\left(\widetilde{x}, U_{V}\right)$, where $\widetilde{x} \in V_{4}$ and $U_{V} \in G_{V}$, the total bundle space $\widetilde{P}$ is a smooth manifold, the surjection $\widetilde{s}$ is a smooth map $\widetilde{s}: \widetilde{P} \rightarrow V_{4}$. The collection of matter fields of arbitrary spins $\widetilde{\Phi}(\widetilde{x})$ take values in standard fiber over $\widetilde{x}: \widetilde{s}^{-1}\left(\widetilde{\mathcal{U}}_{i}\right)=\widetilde{\mathcal{U}}_{i} \times \widetilde{F}_{\widetilde{x}}$. The action of the structure group $G_{V}$ on $\widetilde{P}$ defines an isomorphism of the Lie algebra $\widetilde{\mathfrak{g}}$ of $G_{V}$ onto the Lie algebra of vertical vector fields on $\widetilde{P}$ tangent to the fiber at each $\widetilde{p} \in \widetilde{P}$ called fundamental. The structure group $G_{V}$ is generated by the hidden local internal symmetry of two-parameter abelian local group $U^{\text {loc }}(2)=U(1)_{Y} \times \bar{U}(1) \equiv U(1)_{Y} \times \operatorname{diag}[S U(2)]$. The latter is implemented on the flat space, $M_{4}$. Involving a drastic revision of the role of gauge fields in the physical concept of the spacetime deformation, we generalize the standard gauge scheme by exploring a new special type of distortion gauge field $a(x)\left(\equiv a_{n}(x)\right)$. This field takes values in the Lie algebra of the abelian group $U^{l o c}(2)$ and acts on the external spacetime groups. Besides, we also consider the principle fiber bundle, $P\left(M_{4}, U^{l o c}(2) ; s\right)$, with the base space $M_{4}$, the structure group $U^{l o c}(2)$ and the surjection $s$. Hereinafter the quantities referring to $V_{4}$ are denoted by wiggles, and are left without wiggles if they correspond to $M_{4}$. The matter fields $\Phi(x)$ take values in the standard fiber which is the Hilbert vector space where a linear representation $U(x)$ of group $U^{l o c}(2)$ is given. This space can be regarded as the Lie algebra of the group $U^{l o c}(2)$ upon which the Lie algebra acts according to the law of the adjoint representation: $a \leftrightarrow$ ad $a \Phi \rightarrow[a, \Phi]$.

Third step: The physical system of the fields $\widetilde{\Phi}(\widetilde{x})$ defined on $V_{4}$ must be invariant under the finite local gauge transformations $U_{V}$ of the Lie group of gravitation $G_{V}: \widetilde{\Phi}^{\prime}(\widetilde{x})=U_{V}(\widetilde{x}) \widetilde{\Phi}(\widetilde{x})$, and $\left[\widetilde{\gamma}^{\mu}(\widetilde{x}) \widetilde{\nabla}_{\mu} \widetilde{\Phi}(\widetilde{x})\right]^{\prime}=U_{V}(\widetilde{x})\left[\widetilde{\gamma}^{\mu}(\widetilde{x}) \widetilde{\nabla} \widetilde{\Phi} \widetilde{x}(\widetilde{x})\right]$. The invariance of the Lagrangian of matter fields, $L_{\widetilde{\Phi}}$, under the infinite-parameter group of general covariance in $V_{4}$ implies an invariance of $L_{\widetilde{\Phi}}$ under the $G_{V}$ group and vice versa if, and only if, the generalized local gauge transformations of the fields $\widetilde{\Phi}(\widetilde{x})$ and their covariant derivative $\widetilde{\nabla}_{\mu} \widetilde{\Phi}(\widetilde{x})$ are introduced by finite local $U_{V} \in G_{V}$ gauge transformations. The GGP scheme is

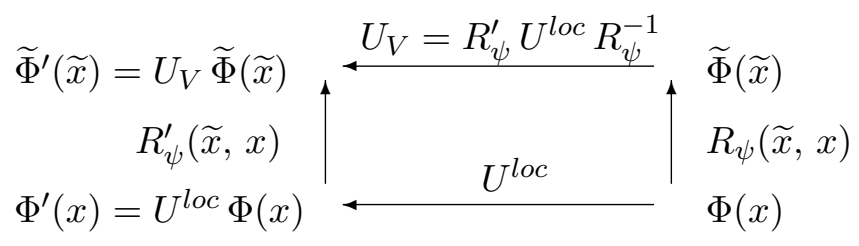

Fourth step: In order the gauge transformations to be universally satisfied, we consider a following smooth unitary map of all the matter fields and their covariant derivatives: $R_{\psi}(a): \Phi \rightarrow \widetilde{\Phi}$, and 

notes the gauge invariant scalar function. Reviewing notations the $\widetilde{\nabla}_{\mu}$ denotes the covariant derivative agreed with the metric, $\widetilde{g}^{\mu \nu}=(1 / 2)\left(\widetilde{\gamma}^{\mu} \widetilde{\gamma}^{\nu}+\widetilde{\gamma}^{\nu} \widetilde{\gamma}^{\mu}\right): \widetilde{\nabla}_{\mu}=\widetilde{\partial}_{\mu}+\widetilde{\Gamma}_{\mu}$, where $\widetilde{\Gamma}_{\mu}(\widetilde{x})=\frac{1}{2} J^{a b} \widetilde{e}_{a}^{\nu}(\widetilde{x}) \widetilde{\partial}_{\mu} \widetilde{e}_{b \nu}(\widetilde{x})$ is the connection, $J_{a b}$ are the generators of Lorentz group $\Lambda$. The tetrad components $\widetilde{e}_{a}^{\mu}(\widetilde{x})$ associate with the chosen representation $D(\Lambda)$ by which the $\widetilde{\Phi}(\widetilde{x})$ is transformed as $[D(\Lambda)]_{l \cdots k}^{l^{\prime} \ldots k^{\prime}} \widetilde{\Phi}(\widetilde{x})$, where $D(\Lambda)=I+\frac{1}{2} \widetilde{\omega}^{a b} J_{a b}, \quad \widetilde{\omega}_{a b}=-\widetilde{\omega}_{b a}$ are the parameters of the Lorentz group. One has, for example, to set $\widetilde{\gamma}^{\mu}(\widetilde{x}) \rightarrow \widetilde{e}^{\mu}(\widetilde{x})$ for the fields of $\operatorname{spin}(j=0,1)$; for vector field $\left[J_{a b}\right]_{k}^{l}=\delta_{a}^{l} \eta_{b k}-\delta_{b}^{l} \eta_{a k}$; but $\widetilde{\gamma}^{\mu}(\widetilde{x})=\widetilde{e}_{a}{ }^{\mu}(\widetilde{x}) \gamma^{a}$ and $J_{a b}=-(1 / 4)\left[\gamma_{a}, \gamma_{b}\right]$ for the spinor field $\left(j=\frac{1}{2}\right)$, where $\gamma^{a}$ are the Dirac matrices. To determine $R(a)$ and $S(F)$, our strategy is as follows: (i) we may obtain the identity by inserting first relation into the second relation; (ii) we further equate in this identity the coefficients in front of $\Phi$ and $\partial \Phi$ to zero. In this way we have the two relations to determine $R(a)$ and $S(F)$. In case of zero curvature, one has $\psi_{l}^{\mu}=D_{l}^{\mu}=e^{\mu}{ }_{l}=\left(\partial x^{\mu} / \partial X^{l}\right), \quad\|D\| \neq 0$, where $X^{l}$ are the inertial coordinates. In this, the conventional gauge theory given on the $M_{4}$ is restored in both curvilinear and inertial coordinates. Although the distortion gauge field $\left(a_{A}\right)$ is a vector field, nevertheless only the gravitational attraction is presented in the proposed theory of gravitation. Although we have explored the simplest abelian symmetry $U^{l o c}$ as a hidden symmetry, however, one may envisage that a straightforward extension should be to achieve the full machinery of the GGP scheme for non-abelian symmetries.

\subsection{Nonlinear realization of the Lie group, $G_{D}$}

We connect the structure group $G_{V}$, further, to the nonlinear realization of the Lie group, $G_{D}$, of distortion of the spacetime, i.e. we extend the curvature of the spacetime continuum to general distortion as the theory of spontaneous breaking of distortion symmetry. The nonlinear realization technique, or the method of phenomenological Lagrangians, provides a way to determine the transformation properties of fields defined on the quotient space. We treat the distortion group $G_{D}$ and its stationary subgroup $H=S O(3)$, respectively, as the dynamical group and its algebraic subgroup. The fundamental field is distortion gauge field and, thus, all the fundamental gravitational structures in fact - the metric as much as the coframes and connections - acquire a distortion-gauge induced theoretical interpretation. Constructing a non-linear realization of the Lie group of distortion $G_{D}$, first, within the scheme of the GGP we necessarily introduce the language of the conceptual six-dimensional geometry of $M_{6}$, which is assumed as a toy model underlying the $M_{4}$. This replacement appears to be indispensable in our discussion of the distortion of the local internal properties of the spacetime continuum. The space $M_{6}$ reads $M_{6}=R_{+}^{3} \oplus R_{-}^{3}=R^{3} \oplus T^{3}, \operatorname{sgn}\left(R^{3}\right)=(+++), \quad \operatorname{sgn}\left(T^{3}\right)=(---)$. The $e_{(\lambda \alpha)}=O_{\lambda} \times \sigma_{\alpha} \quad(\lambda= \pm, \alpha=1,2,3)$ are linearly independent unit basis vectors at the point (p) of interest of the given three-dimensional space $R_{\lambda}^{3}$. The unit vectors $O_{\lambda}$ and $\sigma_{\alpha}$ imply $<O_{\lambda}, O_{\tau}>={ }^{*} \delta_{\lambda \tau}, \quad<\sigma_{\alpha}, \sigma_{\beta}>=\delta_{\alpha \beta}$, where $\delta_{\alpha \beta}$ is the Kronecker symbol, and ${ }^{*} \delta_{\lambda \tau}=1-\delta_{\lambda \tau}$. Three spatial $e_{\alpha}=\xi \times \sigma_{\alpha}$ and three temporal $e_{0 \alpha}=\xi_{0} \times \sigma_{\alpha}$ components are the basis vectors, respectively, in spaces $R^{3}$ and $T^{3}$, where $O_{ \pm}=(1 / \sqrt{2})\left(\xi_{0} \pm \xi\right), \quad \xi_{0}^{2}=-\xi^{2}=1, \quad<\xi_{0}, \xi>=0$. The 3D space $R_{ \pm}^{3}$ is spanned by the coordinates $\eta_{( \pm \alpha)}$. In using the $6 \mathrm{D}$ language, we will be able to make a necessary reduction to the conventional $4 \mathrm{D}$ space which can be achieved in the following way.

(i) In case of free flat space $M_{6}$, the subspace $T^{3}$ is isotropic. And in so far it contributes in line element just only by the square of the moduli $t=\left|\mathbf{x}^{0}\right|, \mathbf{x}^{0} \in T^{3}$, then, the reduction $M_{6} \rightarrow M_{4}=$ $R^{3} \oplus T^{1}$ can be readily achieved if we use $t=\left|\mathbf{x}^{0}\right|$ for conventional time.

(ii) In case of curved space, the reduction $V_{6} \rightarrow V_{4}$ can be achieved if we use the projection $\left(\widetilde{e}_{0}\right)$ of the temporal component $\left(\widetilde{e}_{0 \alpha}\right)$ of basis six-vector $\widetilde{e}\left(\widetilde{e}_{\alpha}, \widetilde{e}_{0 \alpha}\right)$ on the given universal direction $\left(\widetilde{e}_{0 \alpha} \rightarrow \widetilde{e}_{0}\right)$. By this we choose the time coordinate. Actually, the Lagrangian of physical fields defined on $R_{6}$ is a function of scalars such as $A_{(\lambda \alpha)} B^{(\lambda \alpha)}=A_{\alpha} B^{\alpha}+A_{0 \alpha} B^{0 \alpha}$, then upon the reduction of temporal components of six-vectors $A_{0 \alpha} B^{0 \alpha}=A^{0 \alpha}<\widetilde{e}_{0 \alpha}, \widetilde{e}_{0 \beta}>B^{0 \beta}=A^{0}<\widetilde{e}_{0}, \widetilde{e}_{0}>B^{0}=A_{0} B^{0}$ we may fulfill a reduction to $V_{4}$. 


\subsection{Lie group $G_{D}$ of the distortion}

A distortion of the basis comprises the following two steps. We, at first, consider distortion transformations of the ingredient unit vectors $O_{\tau}$ under the distortion gauge field $(a): \widetilde{O}_{(+\alpha)}(a)=$ $\mathcal{Q}_{(+\alpha)}^{\tau}(a) O_{\tau}=O_{+}+æ a_{(+\alpha)} O_{-}, \widetilde{O}_{(-\alpha)}(a)=\mathcal{Q}_{(-\alpha)}^{\tau}(a) O_{\tau}=O_{-}+æ a_{(-\alpha)} O_{+}$, where $\mathcal{Q}\left(=\mathcal{Q}_{(\lambda \alpha)}^{\tau}(a)\right)$ is an element of the group $Q$. This, in turn, induces the distortion transformations of the ingredient unit vectors $\sigma_{\beta}$, which undergo the rotations, $\widetilde{\sigma}_{(\lambda \alpha)}(\theta)=\mathcal{R}_{(\lambda \alpha)}^{\beta}(\theta) \sigma_{\beta}$, where $\mathcal{R}(\theta) \in S O(3)$ is the element of the group of rotations of planes involving two arbitrary axes around the orthogonal third axis in the given ingredient space $R_{\lambda}^{3}$. Then, the resulting basis vectors $\widetilde{\sigma}_{(\lambda \alpha)}(\theta)$ of each three-dimensional ingredient space $R_{\lambda}^{3}$ retain the orthogonality condition between themselves, but violate it with respect to the basis vectors of different ingredient spaces. That is, $\left\langle\widetilde{\sigma}_{(\lambda \alpha)}, \widetilde{\sigma}_{(\tau \beta)}>_{\alpha \neq \beta} \neq 0\right.$, at $\lambda \neq \tau$. In fact, distortion transformations of basis vectors $(O)$ and $(\sigma)$ are not independent, but rather are governed by the spontaneous breaking of the distortion symmetry. To avoid a further proliferation of indices, thenceforth we will use upper case Latin $(A)$ in indexing $(\lambda \alpha) \quad(\lambda= \pm ; \alpha=1,2,3)$, etc. The infinitesimal transformations $\operatorname{read} \delta \mathcal{Q}_{A}^{\tau}(a)=æ \delta a_{A} X_{\lambda}^{\tau} \in Q, \delta \mathcal{R}(\theta)=-\frac{i}{2} M_{\alpha \beta} \delta \omega^{\alpha \beta} \in S O(3)$, provided by the generators $X_{\lambda}^{\tau}={ }^{*} \delta_{\lambda}^{\tau}$ and $I_{i}=\frac{\sigma_{i}}{2}$, where $\sigma_{i}$ are the Pauli matrices, $M_{\alpha \beta}=\varepsilon_{\alpha \beta \gamma} I_{\gamma}$ and $\delta \omega^{\alpha \beta}=\varepsilon_{\alpha \beta \gamma} \delta \theta_{\gamma}$. The transformation matrix $D(a, \theta)=\mathcal{Q}(a) \times \mathcal{R}(\theta)$ is an element of the distortion group $G_{D}=Q \times S O(3): D_{\left(d a^{A}, d \theta^{A}\right)}=I+d D_{\left(a^{A}, \theta^{A}\right)}, d D_{\left(a^{A}, \theta^{A}\right)}=i\left[d a^{A} X_{A}+d \theta^{A} I_{A}\right]$, where $I_{A} \equiv I_{\alpha}$ at given $\lambda$. The generators $X_{A}$ of the group Q do not complete the group $\mathrm{H}$ to the dynamical group $G_{D}$, therefore, they cannot be interpreted as the generators of the quotien space $G_{D} / H$, and the distortion fields $a_{A}$ cannot be identified directly with the Goldstone fields arisen in spontaneous breaking of the distortion symmetry $G_{D}$. These objections, however, can be circumvented, because, as it is shown by (Ter-Kazarian, 2010), the distortion group $G_{D}=Q \times S O(3)$ can be mapped in a one-to-one manner onto the group $G_{D}=S O(3) \times S O(3)$, which is isomorphic to the chiral group $S U(2) \times S U(2)$. The method of phenomenological Lagrangians is well known for this group. Hence we arrive at $\tan \theta_{A}=-\Re a_{A}$. Given the distortion field $a_{A}$, this key relation uniquely determines six angles $\theta_{A}$ of rotations around each of six $(A)$ axes. The fundamental field is distortion gauge field (a) and, thus, all the fundamental gravitational structures in fact - the metric as much as the coframes and connections - acquire a distortion-gauge induced theoretical interpretation. We study the geometrical structure of the space of parameters in terms of Cartan's calculus of exterior forms and derive the Maurer-Cartan structure equations, where the distortion fields (a) are treated as the Goldstone fields (Ter-Kazarian, 2010).

\subsection{A spontaneous breaking of gravitation gauge symmetry}

As alluded to above, in realization of the structure group $G_{V}$ we implement the abelian local group $U^{l o c}=U(1)_{Y} \times \bar{U}(1) \equiv U(1)_{Y} \times \operatorname{diag}[S U(2)]$, on the space $M_{6}$ (spanned by the coordinates $\eta$ ), with the group elements of $\exp \left[i \frac{Y}{2} \theta_{Y}(\eta)\right]$ of $U(1)_{Y}$ and $\exp \left[i T^{3} \theta_{3}(\eta)\right]$ of $\bar{U}(1)$. The group $U^{\text {loc }}(2)$ has two generators, the third component $T^{3}$ of isospin $\vec{T}$ related to the Pauli spin matrix $\frac{\vec{\tau}}{2}$, and hypercharge $Y$ implying $Q^{d}=T^{3}+\frac{Y}{2}$, where $Q^{d}$ is the distortion charge operator assigning the number -1 to particles, but +1 to anti-particles. The group $U^{l o c}(2)$ entails two neutral gauge bosons of $\bar{U}(1)$, or that coupled to $T^{3}$, and of $U(1)_{Y}$, or that coupled to the hypercharge $Y$. The gauge invariant Lagrangian of the fermion field is given in the standard form by $\mathcal{L}=\bar{\psi}(\eta) i \gamma^{A} D_{A} \psi(\eta)$, provided the covariant derivative is $D_{A} \psi(\eta)=\left(\partial_{A}-i g T^{3} W_{A}^{3}-i g^{\prime}(Y / 2) B_{A}\right) \psi(\eta)$, and $g, g^{\prime}$ being the $\bar{U}(1), \quad U(1)_{Y}$ coupling strengths, respectively. Spontaneous symmetry breaking can be achieved by introducing the neutral complex scalar Higgs field (Ter-Kazarian, 2010): $\quad \phi=\left(\begin{array}{c}0 \\ \phi^{0}\end{array}\right), \quad Y(\phi)=1, \quad \phi^{0}=\frac{1}{\sqrt{2}}\left(\phi_{1}+i \phi_{2}\right)$, with the standard potential energy density function $V(\phi)=-\mu^{2} \phi^{+} \phi+\lambda\left(\phi^{+} \phi\right)^{2}$, where $\mu^{2}>0, \quad \lambda>0$. This is an ingredient of the gauge invariant Lagrangian of Higgs field $\mathcal{L}_{H}=\left(D_{A} \phi\right)^{+}\left(D^{A} \phi\right)-V(\phi)$, where $D_{A} \phi(\eta)=\left(\partial_{A}-i g T^{3} W_{A}^{3}-i g^{\prime}(Y / 2) B_{A}\right) \phi(\eta)$. Minimization of the vacuum energy fixes the non-vanishing VEV: $\quad<\phi>_{0} \equiv<0|\phi| 0>=\left(\begin{array}{c}0 \\ \frac{v}{\sqrt{2}}\end{array}\right), \quad v=\left(\frac{\mu^{2}}{\lambda}\right)^{1 / 2}$, leaving one Goldstone boson. The VEV of spontaneously breaks the theory, leaving the $U(1)_{d}$ subgroup intact. The unitary gauge 
$\phi(\eta)=U^{-1}\left(\xi_{3}\right)\left(\begin{array}{c}0 \\ \frac{v+\zeta(\eta)}{\sqrt{2}}\end{array}\right), U\left(\xi_{3}\right)=\exp \left[\frac{i \xi_{3} \cdot \tau^{3}}{v}\right]$, is parameterized by two real shifted fields $\xi_{3}$ and $\zeta$, such that $<0\left|\xi_{3}\right| 0>=<0|\zeta| 0>=0$. The gauge transformation $\phi^{\prime}=U\left(\xi_{3}\right) \phi=\frac{v+\zeta}{\sqrt{2}} \chi, \quad \chi=\left(\begin{array}{c}0 \\ 1\end{array}\right)$, leads to $V\left(\phi^{\prime}\right)=\mu^{2} \zeta^{2}+\lambda v \zeta^{3}+(\lambda / 4) \zeta^{4}$, which gives the mass of Higgs boson $M_{H}=\sqrt{2} \mu$. An examination of the $v^{2}$-terms in the kinetic piece of the Lagrangian $\mathcal{L}_{H}=\left(D_{A} \phi^{\prime}\right)^{+}\left(D^{A} \phi^{\prime}\right)-V\left(\phi^{\prime}\right)$ reveals the mass terms for the physical gauge bosons: $\frac{v^{2}}{2}\left|\left(i \frac{g}{2} \tau^{3} W_{A}^{\prime 3}+i g^{\prime} \frac{Y}{2} B_{A}^{\prime}\right) \chi\right|^{2}=$ $\frac{1}{2}\left(\bar{a}_{A}, a_{A}\right)\left(\begin{array}{cc}M_{\bar{a}}^{2} & 0 \\ 0 & 0\end{array}\right)\left(\begin{array}{c}\bar{a}^{A} \\ a^{A}\end{array}\right)$. The mass matrix can be diagonalized by the standard orthogonal transformations: $\bar{a}_{A}=\cos \theta_{W} W_{A}^{\prime 3}+\sin \theta_{W} B_{A}^{\prime}, a_{A}=\sin \theta_{W} W_{A}^{\prime 3}+\cos \theta_{W} B_{A}^{\prime}, M_{\bar{a}}=\frac{v}{2} \sqrt{g^{2}+g^{\prime 2}}, \quad M_{a}=0$, where $\tan \theta_{W}=g^{\prime} / g$. Namely, the neutral gauge field $W_{A}^{\prime 3}$ mixes with the abelian gauge field $B_{A}^{\prime}$ to form the physical states $\bar{a}_{A}$ and $a_{A}$, with the masses $M_{\bar{a}} \neq 0$ and $M_{a}=0$, respectively. For a neutral current we get $\mathcal{L}_{\text {int }}=æ\left(\mathcal{J}_{A}^{(0)} A^{A}+\mathcal{J}_{A}^{(M)} \bar{a}^{A}\right) \equiv \rightsquigarrow\left(\mathcal{J}_{A} a^{A}\right)$, where $æ=g \sin \theta$, and $\mathcal{J}_{A}^{(0)}=\bar{\psi}(\eta) i \gamma_{A} Q^{d} \psi(\eta), \mathcal{J}_{A}^{(M)}=\bar{\psi}(\eta) i \gamma_{A} Q^{(i n)} \psi(\eta), \mathcal{J}_{A}=\bar{\psi}(\eta) i \gamma_{A} \psi(\eta), \quad Q^{(i n)}=\frac{T^{3}-\sin ^{2} \theta_{W}, Q^{g r}}{\sin \theta_{W} \cos \theta_{W}},$. That is, the left Goldstone boson is gauged away from the scalar sector, but it essentially reappears in the gauge sector providing the longitudinally polarized spin state of one of gauge bosons that acquires mass through its coupling to Higgs scalar. Consequently, the two neutral gauge bosons were mixed to form two physical orthogonal states of the massless component of distortion field, $(a)\left(M_{a}=0\right)$, which is responsible for gravitational interactions, and its massive component, $(\bar{a})\left(M_{\bar{a}} \neq 0\right)$, which is responsible for the ID-regime. Hence, a substantial change of the properties of the spacetime continuum besides the curvature may arise at huge energies. The theory is renormalizable, because gauge invariance gives conservation of charge, also ensures the cancelation of quantum corrections that would otherwise result in infinitely large amplitudes. Without careful thought we expect that in this framework the renormalizability of the theory will not be spoiled in curved space-time too, because, the infinities arise from ultra-violet properties of Feynman integrals in momentum space which, in coordinate space, are short distance properties, and locally (over short distances) all curved space-time look like maximally symmetric (flat) space.

\subsection{The $12 \mathrm{D}$ smooth differentiable manifold $\widetilde{M}_{12}$}

In pursuing our goal further, we are necessarily led to extend a whole framework of GGP to 12D smooth differentiable manifold $\widetilde{M}_{12}$. Consider the curve $\lambda(t): R^{1} \rightarrow \widetilde{M}_{12}$ passing through the point $p=\lambda(0) \in \widetilde{M}_{12}$ with tangent vector $\left.\mathbf{A}\right|_{\lambda(t)}$. The $\{\zeta\}$ are local coordinates in open neighborhood of $p \in \mathcal{U}$. The 12-dimensional smooth vector field $\mathbf{A}_{p}=\mathbf{A}(\zeta)$ belongs to the section of tangent bundle $\mathbf{T}_{p}$ at the point $p(\zeta)$. The one parameter group of diffeomorphisms $A^{t}$ for the curve $\zeta(t)$ passing through point $p$ and $\zeta(0)=\zeta_{p}, \quad \dot{\zeta}(0)=\mathbf{A}_{p}$, is written as $d A_{p}^{t}(\mathbf{A})=\left.\frac{d}{d t}\right|_{t=0} A^{t}(\zeta(t))=\mathbf{A}_{p}(\zeta)$, such that $d A^{t}: \mathbf{T}\left(\widetilde{M}_{12}\right) \rightarrow R\left(\mathbf{T}\left(\widetilde{M}_{12}\right)=\bigcup_{p(\zeta)} \mathbf{T}_{p}\right)$. The $e_{(\lambda, \mu, \alpha)}=O_{\lambda, \mu} \times \sigma_{\alpha} \quad(\lambda, \mu=1,2 ; \quad \alpha=1,2,3)$ are linear independent 12 unit basis vectors at the point $p \in \widetilde{M}_{12}$ provided by the unit vectors $O_{\lambda, \mu}$ and $\sigma_{\alpha}$ implying $<O_{\lambda, \mu}, O_{\tau, \nu}>={ }^{*} \delta_{\lambda \tau}{ }^{*} \delta_{\mu \nu}, \quad<\sigma_{\alpha}, \sigma_{\beta}>=\delta_{\alpha \beta}$, where $\delta_{\alpha \beta}$ is the Kronecker symbol, but ${ }^{*} \delta_{11}={ }^{*} \delta_{22}=0$ and ${ }^{*} \delta_{12}={ }^{*} \delta_{21}=1$. The basis vectors $\sigma_{\alpha}$ refer to given three dimensional ordinary space $R_{\lambda \mu}^{3}$. The metric on $\widetilde{M}_{12}$ reads $\hat{\mathbf{g}}: \mathbf{T}_{p} \times \mathbf{T}_{p} \rightarrow C^{\infty}\left(\widetilde{M}_{12}\right)$. Let also the massless gauge field $a_{A}(\zeta)$ associates with connection in principle bundle $p: E \rightarrow M_{12}$ with a structure group $U^{l o c}$, where the coordinates $\zeta$ exist in the whole region $\mathcal{U}$ of space $M_{12}=M_{6} \oplus \bar{M}_{6}$. The $M_{6}$ relates to the spacetime, but the $\bar{M}_{6}$ is displayed as a space of inner degrees of freedom. In the framework of method of phenomenological Lagrangians, we address the non-linear realizations of the Lie group $G$ of distortion of 12-dimensional space. One may now implement the matrix $D(a, \theta)=\mathcal{Q}(a) \times \mathcal{R}(\theta)$ of distortion group $G=Q \times S O(3)$ by which the basis vector $e$ is transformed at point $p \in \widetilde{M}_{12}$. The infinitesimal transformations read $\delta \mathcal{Q}_{A}^{\tau, \nu}(a)=æ \delta a_{A} X_{\lambda \mu}^{\tau \nu} \in Q, \quad \delta \mathcal{R}(\theta)=-\frac{i}{2} M_{\alpha \beta} \delta \omega^{\alpha \beta} \in S O(3)$, provided by the generators $X_{\lambda \mu}^{\tau \nu}={ }^{*} \delta_{\lambda}^{\tau^{*}} \delta_{\mu}^{\nu}$, and $I_{i}=\frac{\sigma_{i}}{2}$, where $\sigma_{i}$ are the Pauli's matrices, such that $M_{\alpha \beta}=\varepsilon_{\alpha \beta \gamma} I_{\gamma}, \quad\left[\frac{\sigma_{i}}{2}, \frac{\sigma_{k}}{2},\right]=i \varepsilon_{i k j} \frac{\sigma_{j}}{2}, \quad \delta \omega^{\alpha \beta}=\varepsilon_{\alpha \beta \gamma} \delta \theta_{\gamma}$. Consequenttly, the infinitesimal action of the group on basis vectors $e_{A}$ can be written as $d e_{A}=e_{A} d F_{A}=O_{A}(d) \times \sigma_{A}+O_{A} \times \sigma_{A}(d)=$ $i\left[\omega^{A}(d) X_{A}+\vartheta^{A}(d) I_{A}\right] e_{A}$, where we denote $e_{A} \equiv\left(\exp F_{A}\right)$. It is shown that the distortion group 
$G=Q \times S O(3)$ can be mapped onto the group $G=S O(3) \times S O(3)$ in one-to-one manner. The latter is isomorphic to the isotopic chiral group $S U(2) \times S U(2)$, for which the method of phenomenological Lagrangians is well known. For the sake of simplicity, throughout this item we leave the Greek indices implicit unless otherwise stated, such that $A=(\lambda \mu i) \rightarrow i=1,2,3$, . But it goes without saying that all the results obtained refer to the given three-dimensional ingredient space $R_{\lambda \mu}^{3}$. Three $I_{i}$ among the six generators of the group correspond to isotopic transformations, and three $K_{i^{-}}$to special chiral transformations mixing the states of different parities. They imply the conventional commutation relations of invariant subgroup $H=S O(3)$, with the generators $I_{i}$, and of quotien space $G / H$, with the generators $K_{i}$, where $\varepsilon_{i j k}$ denotes the antisymmetric unit tensor. Three parameters $\bar{a}^{i}(a)$ of special chiral transformations, with respect to which the Lagrangian of physical fields is not invariant, can be identified with three Goldstone fields. Consequently we arrive at $\tan \theta_{A}=-æ a_{A}$. This key relation contains no new information, beyond the fact that it uniquely determines the 12 angles $\theta_{A}$ of rotations around each of 12 axes $(A)$ at given distortion field $a_{A}$. The Maurer-Cartan's structure equations $\left(\omega^{i}\right)^{\prime}=\left[\omega^{k}, \omega_{k}^{i}\right]$, and $\left(\omega_{k}^{i}\right)^{\prime}=-R_{j k i}^{l}\left[\omega^{k}, \omega^{i}\right] / 2+\left[\omega_{j}^{k}, \omega_{k}^{l}\right]$, describe the motion of the orthogonal reper joint to the given point of the group space, where $R_{j k i}^{l}=-\varepsilon_{l j \gamma} \varepsilon_{\gamma k i}$ is the curvature of the group space. The forms $\omega^{i}$ and $\omega_{k}^{i}$ are interpreted as the transformations of translation and rotation of the orthogonal reper, such that the rotation $\left(\vartheta^{l} I_{l}\right)$ belongs to stationary group $H$ while the translation reads as $\left(\omega^{i} K_{i}\right)$. Whereas, the invariant constraint means that there always exists the rotation transformation of stationary subgroup $H$ annulling the change in equation of Cartan's forms arisen from the translation transformation of the quotien space $\bar{Q}=G / H$. A general transformation of the group $G$ can be written as $G=\bar{Q}(\bar{a}) H(\theta)$, where $\bar{Q}(\bar{a})$ is the transformation belonged to the left adjacent class $G / H$ of the group $G$ by subgroup $H$.

\subsection{The extended field equations}

The Lagrangian of Goldstone field $(\bar{a})$ can be identified with the square of interval of the geodesic line, with minimal number of derivatives, between the infinitely closed points $\bar{a}^{i}$ and $\bar{a}^{i}+d \bar{a}^{i} L_{\bar{a}}(\zeta)=$ $\frac{1}{2} \omega^{i}\left(\bar{a}, \partial_{A} \bar{a}\right) \omega^{i}\left(\bar{a}, \partial_{A} \bar{a}\right)$, where $\varepsilon_{\alpha i l} \varepsilon_{l \alpha j}=-\delta_{i j}$ is the metric tensor of the group space. Since the massless gauge field $(a)$ is associated with the gauge group $U^{l o c}$, the Lagrangian should be equated to undegenerated Killing form defined on the Lie algebra of the group $U^{l o c}$ in adjoint representation $L_{\bar{a}}(\zeta)=L_{a}(\zeta)=-\frac{1}{4}<F_{A B}(a), F^{A B}(a)>_{K}$, where $F_{A B}(a)$ is the antisymmetric tensor of the gauge field $(a)$. Thence the Goldstone field $(\bar{a})$ as a function of the gauge field $(a)$ can be determined. The covariant derivatives of the matter fields $\Psi$ interacting with the Goldstone fields $\bar{a}$ are determined by means of the form $\vartheta^{\alpha} L=L_{0}\left(\Psi, \partial_{A} \Psi+\vartheta^{\alpha}\left(\bar{a}, \partial_{A} \bar{a}\right) T_{\alpha} \Psi\right)$, where $L_{0}\left(\Psi, \partial_{A} \Psi\right)$ is the Lagrangian of interacting matter fields classified by the linear representations $T_{\alpha}$ of the subgroup $H$. The Lagrangians above are invariant with respect to distortion translations and rotations. These also should be completed by the transformations $\Psi^{\prime}=\left(\exp \left[i \eta^{\alpha}(\bar{a}, g) T_{\alpha}\right]\right) \Psi$.

The extended field equations followed at once in terms of Euler-Lagrange variations respectively on the $M_{12}$ and $\widetilde{M}_{12}$ (Ter-Kazarian, 1986, 1989c, 1997, 2010, 2012): $S=S_{a}+S_{\widetilde{\Phi}}=\int \sqrt{-\eta} L_{a} d^{12} \zeta+$ $\int \sqrt{-g} L_{\widetilde{\Phi}} d^{12} \widetilde{\zeta}$, where $L_{a}$ is the Lagrangian of distortion field $(a), L_{\widetilde{\Phi}}$ is the Lagrangian of matter fields, whereas the dependence on the distortion gauge field comes only through the components of the metrical tensor. The $L_{a}$ is invariant under Lorentz $(\Lambda)$ and $U^{l o c}$ gauge groups. The Lagrangian $L_{\widetilde{\Phi}}$, in turn, is invariant under the gauge group of gravitation, $G_{R}$. In terms of the Euler-Lagrange variations in $M_{12}$ and $\widetilde{M}_{12}$, we readily obtain $\frac{\delta\left(\sqrt{-\eta} L_{a}\right)}{\delta a_{A}}=-\frac{\partial g^{B C}}{\partial a_{A}} \frac{\delta\left(\sqrt{-g} L_{\widetilde{\Phi}}\right)}{\delta g^{B C}}=-\frac{\sqrt{-g}}{2} \frac{\partial g^{B C}}{\partial a_{A}} \widetilde{T}_{B C}, \quad \frac{\delta \widetilde{L}_{\widetilde{\Phi}}}{\delta \widetilde{\Phi}}=$ $0, \quad \frac{\delta L_{\widetilde{\Phi}}}{\delta \widetilde{\Phi}}=0$, where $\widetilde{T}_{B C}$ is the energy-momentum tensor of the matter fields $\widetilde{\Phi}(\widetilde{\zeta})$. The Lagrangian of distortion gauge field $a_{A}=\left(a_{(\lambda \alpha)}, \bar{a}_{(\tau \beta)}\right)$ defined on the flat space, yields $\partial^{B} \partial_{B} a_{A}-\left(1-\zeta_{0}^{-1}\right) \partial_{A} \partial^{B} a_{B}=$ $J_{A}=-\frac{1}{2} \sqrt{g} \frac{\partial g^{B C}}{\partial a_{A}} \widetilde{T}_{B C}$, where $F_{A B}(a)$ is the antisymmetrical tensor of gauge field $(a), \zeta_{0}$ is the gauge fixing parameter. To render our discussion here more transparent, below we clarify the relation between gravitational and coupling constants. To assist in obtaining actual solutions from the field equations, we may consider the weak-field limit and shall envisage that the right hand side of should be in the form $-\frac{1}{2}\left(4 \pi G_{N}\right) \sqrt{g(x)} \frac{\partial g^{B C}(x)}{\partial x_{A}} \widetilde{T}_{B C}$. Hence, we may assign to the Newton's gravitational constant $G_{N}$ the value $G_{N}=æ^{2} / 4 \pi$. 
Let consider, particularly, the static and spherically symmetrical physical system. In accord, the equation of distortion gauge field $a_{A}=\left(a_{(\lambda \alpha)}, \bar{a}_{(\tau \beta)}\right)$ reads $\partial^{B} \partial_{B} a_{A}-\left(1-\zeta_{0}^{-1}\right) \partial_{A} \partial^{B} a_{B}=$ $J_{A}=-\frac{1}{2} \sqrt{g} \frac{\partial g^{B C}}{\partial a_{A}} T_{B C}$, where $T_{B C}$ is the energy-momentum tensor, $\zeta_{0}$ is the gauge fixing parameter. To render our discussion here more transparent, below we clarify the relation between gravitational and coupling constants. To assist in obtaining actual solutions from the field equations, we may consider the weak-field limit and shall envisage that the right hand side should be in the form $-\frac{1}{2}\left(4 \pi G_{N}\right) \sqrt{g(x)} \frac{\partial g^{B C}(x)}{\partial x_{A}} \widetilde{T}_{B C}$. Hence, we may assign to the Newton's gravitational constant $G_{N}$ the value $G_{N}=æ^{2} / 4 \pi$. The curvature of manifold $M_{6} \rightarrow \widetilde{M}_{6}$ is the familiar distortion induced by the extended field components $a_{(1,1, \alpha)}=a_{(2,1, \alpha)} \equiv \frac{1}{\sqrt{2}} a_{(+\alpha)}, a_{(1,2, \alpha)}=a_{(2,2, \alpha)} \equiv \frac{1}{\sqrt{2}} a_{(-\alpha)}$. The other regime of ID presents at: $a_{(1,1, \alpha)}=-a_{(2,1, \alpha)} \equiv \frac{1}{\sqrt{2}} \bar{a}(+\alpha), a_{(1,2, \alpha)}=-a_{(2,2, \alpha)} \equiv \frac{1}{\sqrt{2}} \bar{a}_{(-\alpha)}$.

\subsection{A hard look at spacetime deformation}

The holonomic metric on $\widetilde{\mathcal{M}}_{4}$ can be recast in the form $\widetilde{g}=\widetilde{g}_{\mu \nu} \widetilde{\vartheta}^{\mu} \otimes \widetilde{\vartheta}^{\nu}=\widetilde{g}\left(\widetilde{e}_{\mu}, \widetilde{e}_{\nu}\right) \widetilde{\vartheta}^{\mu} \otimes \widetilde{\vartheta}^{\nu}$, with components, $\widetilde{g}_{\mu \nu}=\widetilde{g}\left(\widetilde{e}_{\mu}, \widetilde{e}_{\nu}\right)$ in the dual holonomic base $\left\{\widetilde{\vartheta}^{\mu} \equiv d \widetilde{x}^{\mu}\right\}$. It is well known that the notions of space and connections should be separated. The curvature and torsion are in fact properties of a connection, and many different connections are allowed to exist in the same spacetime. Therefore, when considering several connections with different curvature and torsion, one takes spacetime simply as a manifold, and connections as additional structures.

In order to relate local Lorentz symmetry to more general deformed spacetime, there is, however, a need to introduce the soldering tools, which are the linear frames and forms in tangent fiber-bundles to the external a general smooth differential manifold, whose components are so-called tetrad (vierbein) fields. The $\widetilde{\mathcal{M}}_{4}$ has at each point a tangent space, $\widetilde{T}_{\widetilde{x}} \widetilde{M}_{4}$, spanned by the anholonomic orthonormal frame field, $\widetilde{e}$, as a shorthand for the collection of the 4 -tuplet $\left(\widetilde{e}_{0}, \cdots, \widetilde{e}_{3}\right)$, where $\widetilde{e}_{a}=\widetilde{e}_{a}{ }^{\mu} \widetilde{\partial}_{\mu}$. The $\widetilde{\vartheta}^{0}$

frame field, $\widetilde{e}$, then defines a dual vector, $\widetilde{\vartheta}$, of differential forms, $\widetilde{\vartheta}=(\quad \vdots \quad)$, as a shorthand for the $\widetilde{\vartheta}^{3}$

collection of the $\widetilde{\vartheta}^{b}=\widetilde{e}^{b}{ }_{\mu} d \widetilde{x}^{\mu}$, whose values at every point form the dual basis, such that $\left.\widetilde{e}_{a}\right\rfloor \widetilde{\vartheta}^{b}=\delta_{a}^{b}$. In components $\widetilde{e}_{a}{ }^{\mu} \widetilde{e}^{b}{ }_{\mu}=\delta_{a}^{b}$.

On the manifold, $\widetilde{\mathcal{M}}_{4}$, the tautological tensor field, $i \widetilde{d}$, of type $(1,1)$ can be defined which assigns to each tangent space the identity linear transformation. Thus for any point $\widetilde{x} \in \widetilde{\mathcal{M}}_{4}$, and any vector $\widetilde{\xi} \in \widetilde{T}_{\widetilde{x}} \widetilde{\mathcal{M}}_{4}$, one has $i \widetilde{d}(\widetilde{\xi})=\widetilde{\xi}$. In terms of the frame field, the $\widetilde{\vartheta}^{a}$ give the expression for $i \widetilde{d}$ as $i \widetilde{d}=\widetilde{e} \widetilde{\vartheta}=\widetilde{e}_{0} \otimes \widetilde{\vartheta}^{0}+\cdots \widetilde{e}_{3} \otimes \widetilde{\vartheta}^{3}$, in the sense that both sides yield $\widetilde{\xi}$ when applied to any tangent vector $\widetilde{\xi}$ in the domain of definition of the frame field. One can also consider general transformations of the linear group, $G L(4, R)$, taking any base into any other set of four linearly independent fields. The notation, $\left\{\widetilde{e}_{a}, \widetilde{\vartheta}^{b}\right\}$, will be used below for general linear frames.

Let us introduce the so-called first deformation matrices, $\left(\pi(x)_{k}^{m}\right.$ and $\left.\widetilde{\pi}_{l}^{a}(\widetilde{x})\right) \in G L(4, \widetilde{M}) \forall \widetilde{x}$, as follows: $\widetilde{D}_{\mu}^{m}=\widetilde{e}_{\mu}{ }^{k} \pi_{k}^{m}, \quad \widetilde{\psi}_{l}^{\mu}=\widetilde{e}_{k}^{\mu} \pi_{l}^{k}, \quad \widetilde{e}_{\mu}{ }^{k} \widetilde{e}^{\mu}{ }_{m}=\delta_{m}^{k}, \widetilde{\pi}_{a}{ }^{m}=\widetilde{e}_{a}{ }^{\mu} \widetilde{D}_{\mu}^{m}, \quad \widetilde{\pi}^{a}{ }_{l}=\widetilde{e}^{a}{ }_{\mu} \widetilde{\psi}_{l}^{\mu}$, where $\widetilde{g}_{\mu \nu} \widetilde{e}_{k}^{\mu} \widetilde{e}_{s}{ }^{\nu}=\eta_{k s}, \eta_{k s}$ is the metric on $M_{4}$. Hence the deformation tensor, $\Omega_{l}^{m}=\pi_{k}^{m} \pi_{l}^{k}$, yields local tetrad deformations $\widetilde{e}_{a}=\widetilde{\pi}_{a}^{m} e_{m}, \quad \widetilde{\vartheta}^{a}=\widetilde{\pi}_{l}^{a} \vartheta^{l}, \bar{e}_{k}=\pi_{k}^{m} e_{m}, \quad \bar{\vartheta}^{k}=\pi_{l}^{k} \vartheta^{l}$, and that $i \widetilde{d}=\widetilde{e}_{a} \otimes \widetilde{\vartheta}^{a}=$ $\bar{e}_{k} \otimes \bar{\vartheta}^{k} \in \widetilde{\mathcal{M}}_{4}$. The first deformation matrices $\pi$ and $\widetilde{\pi}$, in general, give rise to the right cosets of the Lorentz group, i.e. they are the elements of the quotient group $G L(4, \widetilde{M}) / S O(3,1)$.

If we deform the co-tetrad, we have two choices to recast metric as follows: either writing the deformation of the metric in the space of tetrads or deforming the tetrad field: $\widetilde{g}=o_{a b} \widetilde{\vartheta}^{a} \otimes$ $\widetilde{\vartheta}^{b}=o_{a b} \widetilde{\pi}_{l}^{a} \tilde{\pi}^{b}{ }_{m} \vartheta^{l} \otimes \vartheta^{m}=\gamma_{l m} \vartheta^{l} \otimes \vartheta^{m}$. where the second deformation matrix, $\gamma_{l m}$, reads $\gamma_{l m}=$ $o_{a b} \widetilde{\pi}_{l}^{a} \widetilde{\pi}^{b}{ }_{m}$. The deformed metric splits as $\widetilde{g}_{\mu \nu}=\Upsilon^{2} \eta_{\mu \nu}+\widetilde{\gamma}_{\mu \nu}$, where $\Upsilon=\widetilde{\pi}_{a}^{a}=\pi_{k}^{k}$, and $\widetilde{\gamma}_{\mu \nu}=$ $\left(\gamma_{a l}-\Upsilon^{2} o_{a l}\right) \widetilde{e}^{a}{ }_{\mu} \widetilde{e}^{l}{ }_{\nu}=\left(\gamma_{k s}-\Upsilon^{2} \eta_{k s}\right) \widetilde{e}^{k}{ }_{\mu} \widetilde{e}^{s}{ }_{\nu}$. The anholonomic orthonormal frame field, $\widetilde{e}$, relates $\widetilde{g}$ to the tangent space metric, $o_{a b}=\operatorname{diag}(+---)$, by $o_{a b}=\widetilde{g}\left(\widetilde{e}_{a}, \widetilde{e}_{b}\right)=\widetilde{g}_{\mu \nu} \widetilde{e}_{a}{ }^{\mu} \widetilde{e}_{b}{ }^{\nu}$, which has the converse $\widetilde{g}_{\mu \nu}=o_{a b} \widetilde{e}^{a}{ }_{\mu} \widetilde{e}^{b}{ }_{\nu}$ because $\widetilde{e}_{a}{ }^{\mu} \widetilde{e}^{a}{ }_{\nu}=\delta_{\nu}^{\mu}$. With this provision, we build up a world-deformation tensor $\Omega$ yielding a deformation of the flat space $M_{4}$. The $\gamma_{l m}$ can be decomposed in terms of symmetric, $\widetilde{\pi}_{(a l)}$, and antisymmetric, $\widetilde{\pi}_{[a l]}$, parts of the matrix $\widetilde{\pi}_{a l}=o_{a c} \widetilde{\pi}_{l}^{c}$ (or respectively in terms of $\pi_{(k l)}$ and $\pi_{[k l]}$, 
where $\left.\pi_{k l}=\eta_{k s} \pi^{s}{ }_{l}\right)$ as $\gamma_{a l}=\widetilde{\Upsilon}^{2} o_{a l}+2 \widetilde{\Upsilon} \widetilde{\Theta}_{a l}+o_{c d} \widetilde{\Theta}^{c}{ }_{a} \widetilde{\Theta}^{d}{ }_{l}+o_{c d}\left(\widetilde{\Theta}^{c}{ }_{a} \widetilde{\varphi}^{d}{ }_{l}+\widetilde{\varphi}^{c}{ }_{a} \widetilde{\Theta}^{d}{ }_{l}\right)+o_{c d} \widetilde{\varphi}^{c}{ }_{a} \widetilde{\varphi}^{d}{ }_{l}$, where $\tilde{\pi}_{a l}=\widetilde{\Upsilon}_{a l}+\widetilde{\Theta}_{a l}+\widetilde{\varphi}_{a l}, \widetilde{\Upsilon}=\widetilde{\pi}_{a}^{a}, \widetilde{\Theta}_{a l}$ is the traceless symmetric part and $\widetilde{\varphi}_{a l}$ is the skew symmetric part of the first deformation matrix. The anholonomy objects defined on the tangent space, $\widetilde{T}_{\widetilde{x}} \widetilde{M}_{4}$, $\operatorname{read} \widetilde{C}^{a}:=d \widetilde{\vartheta}^{a}=\frac{1}{2} \widetilde{C}_{b c}^{a} \widetilde{\vartheta}^{b} \wedge \widetilde{\vartheta}^{c}$, where the anholonomy coefficients, $\widetilde{C}_{b c}^{a}$, which represent the curls of the base members, are $\widetilde{C}^{c}{ }_{a b}=-\widetilde{\vartheta}^{c}\left(\left[\widetilde{e}_{a}, \widetilde{e}_{b}\right]\right)=\widetilde{e}_{a}{ }^{\mu} \widetilde{e}_{b}{ }^{\nu}\left(\widetilde{\partial}_{\mu} \widetilde{e}^{c}{ }_{\nu}-\widetilde{\partial}_{\nu} \widetilde{e}^{c}{ }_{\mu}\right)=-\widetilde{e}^{c}{ }_{\mu}\left[\widetilde{e}_{a}\left(\widetilde{e}_{b}{ }^{\mu}\right)-\widetilde{e}_{b}\left(\widetilde{e}_{a}{ }^{\mu}\right)\right]=$ $2 \pi^{c}{ }_{l} \widetilde{e}_{m}^{\mu}\left(\pi^{-1}{ }_{[a}^{m} \widetilde{\partial}_{\mu} \pi_{b]}^{-1}{ }_{b]}\right)$. In particular case of constant metric in the tetradic space, the deformed connection can be written as $\widetilde{\Gamma}_{b c}^{a}=\frac{1}{2}\left(\widetilde{C}_{b c}^{a}-o^{a a^{\prime}} o_{b b^{\prime}} \widetilde{C}_{a^{\prime} c}^{b^{\prime}}-o^{a a^{\prime}} o_{c c^{\prime}} \widetilde{C}^{c^{\prime}}{ }_{a^{\prime} b}\right)$. All magnitudes related to the $V_{4}$ will be denoted with an over ' $\circ$ '. Now we have $\stackrel{\circ}{\Omega}_{l}^{m}=\stackrel{\circ}{D}_{\mu}^{m} \stackrel{\circ}{\psi}_{l}^{\mu}$ and $\breve{\Omega}^{\mu}{ }_{\nu}=\breve{D}_{\rho}^{\mu} \breve{\psi}_{\nu}^{\rho}$, provided $\stackrel{\circ}{e}_{\mu}=\stackrel{\circ}{D}_{\mu}^{l} e_{l}, \stackrel{\circ}{e}_{\mu} \stackrel{\circ}{\psi}_{l}^{\mu}=\stackrel{\circ}{\Omega}{ }_{l}^{m} e_{m}, \widetilde{e}_{\rho}=\breve{D}_{\rho}^{\mu} \stackrel{\circ}{e}_{\mu}, \widetilde{e}_{\rho} \breve{\psi}_{\nu}^{\rho}=\breve{\Omega}^{\mu}{ }_{\nu} \stackrel{\circ}{e}_{\mu}$. The following relations hold: $\stackrel{\circ}{D}_{\mu}^{m}=\stackrel{\circ}{e}_{\mu}^{k} \stackrel{\circ}{\pi}{ }_{k}, \quad \stackrel{\circ}{\psi}_{l}^{\mu}=\stackrel{\circ}{e}_{k}^{\mu} \stackrel{\circ}{\pi}_{l}^{k}, \stackrel{\circ}{e}_{\mu}^{k} \stackrel{\circ}{e}_{m}^{\mu}=\delta_{m}^{k}, \quad \stackrel{\circ}{\pi}_{a}^{m}=\stackrel{\circ}{e}_{a}^{\mu} \stackrel{\circ}{D}_{\mu}^{m}, \stackrel{\circ}{\pi}_{l}^{a}{ }_{l}=\stackrel{\circ}{e}^{a}{ }_{\mu} \stackrel{\circ}{\psi}_{l}^{\mu}$, where $\stackrel{\circ}{\Omega}_{l}^{m}=\stackrel{\circ}{\pi}$ $m_{\rho} \stackrel{\circ}{\pi}_{l}^{\rho}, \breve{\Omega}^{\mu}{ }_{\nu}=\breve{\pi}_{\rho}^{\mu} \breve{\pi}_{\nu}^{\rho}$. So, $\stackrel{\circ}{g}_{\mu \nu} \stackrel{\circ}{e}_{k}^{\mu} \stackrel{\circ}{e}_{s}^{\nu}=\eta_{k s}$, and $\breve{D}_{\rho}^{\mu}=\breve{e}_{\nu}{ }^{\mu} \breve{\pi}_{\rho}^{\nu}, \quad \breve{\psi}_{\nu}^{\rho}=\breve{e}_{\mu}^{\rho} \breve{\pi}_{\nu}^{\mu}, \quad \breve{e}_{\nu}{ }^{\mu} \breve{e}_{\rho}^{\nu}=\delta_{\rho}^{\mu}$, $\breve{\pi}_{a}{ }^{\mu}=\breve{e}_{a}{ }^{\rho} \breve{D}_{\rho}^{\mu}, \quad \breve{\pi}^{a}{ }_{\nu}=\breve{e}^{a}{ }_{\rho} \breve{\psi}_{\nu}^{\rho}$. The norm $d \stackrel{\circ}{s} \equiv i \stackrel{\circ}{d}$ of the infinitesimal displacement $d \stackrel{\circ}{x}{ }^{\mu}$ on the $V_{4}$ can be written in terms of the spacetime structures of $M_{4}$ as $i \stackrel{\circ}{d}=\stackrel{\circ}{=} \stackrel{\circ}{\vartheta}=\stackrel{\circ}{\Omega}{ }_{l}^{m} e_{m} \otimes \vartheta^{l} \in V_{4}$. The

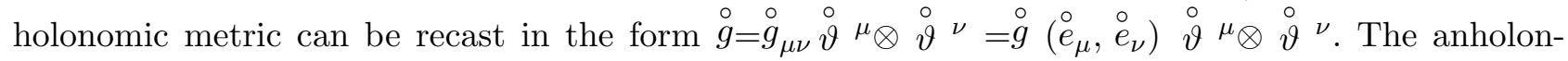
omy objects defined on the tangent space, $\stackrel{\circ}{T}_{x}^{\circ} V_{4}, \operatorname{read} \stackrel{\circ}{C}{ }^{a}:=d \stackrel{\circ}{\vartheta}^{a}=\frac{1}{2} \stackrel{\circ}{C}_{b c}^{a} \stackrel{\circ}{\vartheta}^{b} \wedge \stackrel{\circ}{\vartheta}^{c}$, where the anholonomy coefficients, $\stackrel{\circ}{C}_{b c}^{a}$, which represent the curls of the base members, are $\stackrel{\circ}{C}$

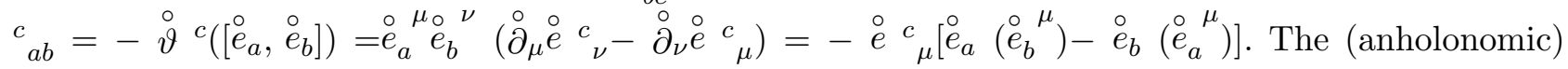
Levi-Civita (or Christoffel) connection can be written as $\left.\left.\left.\stackrel{\circ}{\Gamma}_{a b}:=\stackrel{\circ}{e}_{[a}\right\rfloor d \stackrel{\circ}{\vartheta}_{b]}-\frac{1}{2}\left(\stackrel{\circ}{e}_{a}\right\rfloor \stackrel{\circ}{e}_{b}\right\rfloor d \stackrel{\circ}{\vartheta_{c}}\right) \wedge \stackrel{\circ}{\vartheta}^{c}$, where $\stackrel{\circ}{\vartheta}_{c}$ is understood as the down indexed 1-form $\stackrel{\circ}{\vartheta}_{c}=o_{c b} \stackrel{\circ}{\vartheta^{b}}$. The norm $i \widetilde{d}$ can then be written in terms of the spacetime structures of $V_{4}$ and $M_{4}: i \widetilde{d}=\widetilde{e} \widetilde{\vartheta}=\widetilde{e}_{\rho} \otimes \widetilde{\vartheta}^{\rho}=\widetilde{e}_{a} \otimes \widetilde{\vartheta}^{a}=\breve{\Omega}^{\mu}{ }_{\nu} \stackrel{\circ}{e}_{\mu}$

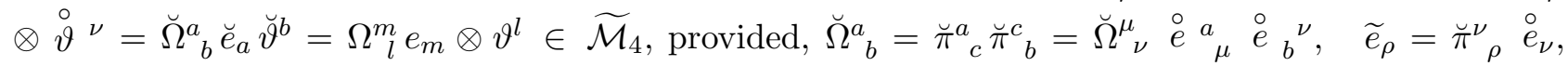
$\widetilde{\vartheta}^{\rho}=\breve{\pi}_{\mu}{ }^{\rho} \stackrel{\circ}{\vartheta}^{\mu}, \quad \widetilde{e}_{c}=\breve{\pi}_{c}{ }^{a} \stackrel{\circ}{e}_{a}, \widetilde{\vartheta}^{c}=\breve{\pi}_{b}^{c}{ }_{b} \stackrel{\circ}{\vartheta}^{b}$. Under a local tetrad deformation, a general spin connection transforms according to $\widetilde{\omega}_{b \mu}^{a}=\breve{\pi}_{c}{ }^{a} \stackrel{\circ}{\omega}^{c}{ }_{d \mu} \breve{\pi}_{b}^{d}+\breve{\pi}_{c}{ }^{a} \widetilde{\partial}_{\mu} \breve{\pi}_{b}^{c}=\pi_{l}{ }^{a} \widetilde{\partial}_{\mu} \pi_{b}^{l}$. We have then two

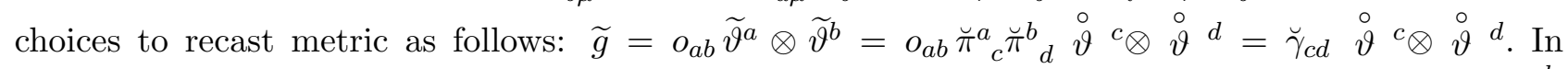
the first case, the contribution of the Christoffel symbols constructed by the metric $\breve{\gamma}_{a b}=o_{c d} \breve{\pi}^{c}{ }_{a} \breve{\pi}_{b}^{d}$

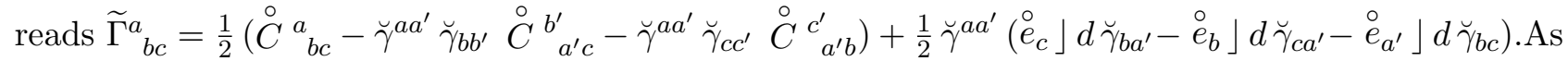
before, the second deformation matrix, $\breve{\gamma}_{a b}$, can be decomposed in terms of symmetric, $\breve{\pi}_{(a b)}$, and antisymmetric, $\breve{\pi}_{[a b]}$, parts of the matrix $\breve{\pi}_{a b}=o_{a c} \breve{\pi}_{b}^{c}$. So, $\breve{\pi}_{a b}=\breve{\Upsilon}_{a b}+\breve{\Theta}_{a b}+\breve{\varphi}_{a b}$, where $\breve{\Upsilon}=\breve{\pi}_{a}^{a}$, $\breve{\Theta}_{a b}$ is the traceless symmetric part and $\breve{\varphi}_{a b}$ is the skew symmetric part of the first deformation matrix. The deformed metric can then be split as $\widetilde{g}_{\mu \nu}(\breve{\pi})=\breve{\Upsilon}^{2}(\breve{\pi}) \stackrel{\circ}{g}_{\mu \nu}+\breve{\gamma}_{\mu \nu}(\breve{\pi})$, where $\breve{\gamma}_{\mu \nu}(\breve{\pi})=$ $\left[\breve{\gamma}_{a b}-\breve{\Upsilon}^{2} o_{a b}\right] \stackrel{\circ}{e}^{a}{ }_{\mu} \stackrel{\circ}{e}^{b}{ }_{\nu}$. The inverse deformed metric reads $\widetilde{g}^{\mu \nu}(\breve{\pi})=o^{c d} \breve{\pi}^{-1 a}{ }_{c} \breve{\pi}^{-1 b}{ }_{d} \stackrel{\circ}{e}{ }_{a}^{\mu} \stackrel{\circ}{e}_{b}{ }^{\nu}$, where $\breve{\pi}^{-1 a}{ }_{c} \breve{\pi}^{c}{ }_{b}=\breve{\pi}^{c}{ }_{b} \breve{\pi}^{-1 a}{ }_{c}=\delta_{b}^{a}$. The (anholonomic) Levi-Civita (or Christoffel) connection can be written as $\left.\left.\left.\widetilde{\Gamma}_{a b}:=\widetilde{e}_{[a}\right\rfloor d \widetilde{\vartheta}_{b]}-\frac{1}{2}\left(\widetilde{e}_{a}\right\rfloor \widetilde{e}_{b}\right\rfloor d \widetilde{\vartheta}_{c}\right) \wedge \widetilde{\vartheta}^{c}$, where $\widetilde{\vartheta}_{c}$ is understood as the down indexed 1-form $\widetilde{\vartheta}_{c}=o_{c b} \widetilde{\vartheta}^{b}$. Hence, the usual Levi-Civita connection is related to the original connection by the relation $\widetilde{\Gamma}_{\rho \sigma}^{\mu}=\breve{\Gamma}_{\rho \sigma}^{\mu}+\breve{\Pi}_{\rho \sigma}^{\mu}$, provided $\Pi_{\rho \sigma}^{\mu}=2 \widetilde{g}^{\mu \nu} \breve{g}_{\nu(\rho} \breve{\nabla}_{\sigma)} \breve{\Upsilon}^{\prime} \breve{g}_{\rho \sigma} g^{\mu \nu} \breve{\nabla}_{\nu} \breve{\Upsilon}+\frac{1}{2} \widetilde{g}^{\mu \nu}\left(\breve{\nabla}_{\rho} \breve{\gamma}_{\nu \sigma}+\breve{\nabla}_{\sigma} \breve{\gamma}_{\rho \nu}-\breve{\nabla}_{\nu} \breve{\gamma}_{\rho \sigma}\right)$, where $\breve{\nabla}$ is the covariant derivative. The contravariant deformed metric, $\widetilde{g}^{\nu \rho}$, is defined as the inverse of $\widetilde{g}_{\mu \nu}$, such that $\widetilde{g}_{\mu \nu} \widetilde{g}^{\nu \rho}=\delta_{\mu}^{\rho}$. Hence, the connection deformation $\Pi_{\rho \sigma}^{\mu}$ acts like a force that deviates the test particles from the geodesic motion in the space, $V_{4}$.

A metric-affine space $\left(\widetilde{M}_{4}, \widetilde{g}, \widetilde{\Gamma}\right)$ is defined to have a metric and a linear connection that need not dependent on each other. In general, the lifting of the constraints of metric-compatibility and symmetry yields the new geometrical property of the spacetime, which are the nonmetricity 1-form $\widetilde{N}_{a b}$ and the affine torsion 2 -form $\widetilde{T}^{a}$ representing a translational misfit. These, together with the curvature 2-form $\widetilde{R}_{a}^{b}$, symbolically can be presented as $\left(\widetilde{N}_{a b}, \widetilde{T}^{a}, \widetilde{R}_{a}^{b}\right) \sim \widetilde{\mathcal{D}}\left(\widetilde{g}_{a b}, \widetilde{\vartheta}^{a}, \widetilde{\Gamma}_{a}^{b}\right)$, where $\widetilde{\mathcal{D}}$ is the covariant exterior derivative. If the nonmetricity tensor $\widetilde{N}_{\lambda \mu \nu}=-\widetilde{\mathcal{D}}_{\lambda} \widetilde{g}_{\mu \nu} \equiv-\widetilde{g}_{\mu \nu ; \lambda}$ does not vanish, the general formula for the affine connection written in the spacetime components is 
Ambartsumian's vision and further insight to key puzzles of ultra-high energy astrophysics

$\widetilde{\Gamma}_{\mu \nu}^{\rho}=\stackrel{\circ}{\Gamma}_{\mu \nu}^{\rho}+\widetilde{K}_{\mu \nu}^{\rho}-\widetilde{N}_{\mu \nu}^{\rho}+\frac{1}{2} \widetilde{N}_{(\mu \nu)}^{\rho}$, where $\stackrel{\circ}{\Gamma}_{\mu \nu}^{\rho}$ is the Riemann part, $\widetilde{K}_{\mu \nu}^{\rho}:=2 \widetilde{Q}_{(\mu \nu)}^{\rho}+\widetilde{Q}_{\mu \nu}^{\rho}$ is the non-Riemann part - the affine contortion tensor. The torsion, $\widetilde{Q}_{\mu \nu}^{\rho}=\frac{1}{2} \widetilde{T}_{\mu \nu}^{\rho}=\widetilde{\Gamma}_{[\mu \nu]}^{\rho}$ given with respect to a holonomic frame, $d \widetilde{\vartheta}^{\rho}=0$, is a third-rank tensor, antisymmetric in the first two indices, with 24 independent components.

In the presence of curvature and torsion, the coupling prescription of a general field carrying an arbitrary representation of the Lorentz group will be $\widetilde{\partial}_{\mu} \rightarrow \widetilde{\mathcal{D}}_{\mu}=\widetilde{\partial}_{\mu}-\frac{i}{2}\left(\widetilde{\omega}_{\mu}^{a b}-\widetilde{K}_{\mu}^{a b}\right) J_{a b}$, with $J_{a b}$ denoting the corresponding Lorentz generator. The Riemann-Cartan manifold, $U_{4}$, is a particular case of the general metric-affine manifold $\widetilde{\mathcal{M}}_{4}$, restricted by the metricity condition $\widetilde{N}_{\lambda \mu \nu}=0$, when a nonsymmetric linear connection is said to be metric compatible. The Lorentz and diffeomorphism invariant scalar curvature, $\widetilde{R}$, becomes either a function of $\widetilde{e}_{\mu}^{a}$ only, or $\widetilde{g}_{\mu \nu}: \widetilde{R}(\widetilde{\omega}) \equiv \widetilde{e}_{a}^{\mu} \widetilde{e}_{b}{ }^{\nu} \widetilde{R}_{\mu \nu}{ }^{a b}(\widetilde{\omega})=$ $\widetilde{R}(\widetilde{g}, \widetilde{\Gamma}) \equiv \widetilde{g}^{\rho \nu} \widetilde{R}_{\rho \mu \nu}^{\mu}(\widetilde{\Gamma})$.

\subsection{Determination of $\widetilde{D}$ and $\widetilde{\psi}$ in standard theory of gravitation}

Let $\widetilde{\omega}^{a b}=\widetilde{\omega}_{\mu}^{a b} \wedge d \widetilde{x}^{\mu}$ be the 1-forms of corresponding connections assuming values in the Lorentz Lie algebra. The action for gravitational field can be written in the form $\widetilde{S}_{g}=\stackrel{\circ}{S}+\widetilde{S}_{Q}$, where the integral $\stackrel{\circ}{S}=-\frac{1}{4 æ} \int \star \stackrel{\circ}{R}=-\frac{1}{4 æ} \int \star \stackrel{\circ}{R}_{c d} \wedge \widetilde{\vartheta}^{c} \wedge \widetilde{\vartheta}^{d}=-\frac{1}{2 æ} \int \stackrel{\circ}{R} \sqrt{-\widetilde{g}} d \Omega$, is the usual Einstein action, with the coupling constant relating to the Newton gravitational constant $æ=8 \pi G_{N} / c^{4}, S_{Q}$ is the phenomenological action of the spin-torsion interaction, $\star$ denotes the Hodge dual. This is a $C^{\infty}$ linear map $\star: \Omega^{p} \rightarrow \Omega^{n-p}$, which acts on the wedge product monomials of the basis 1 -forms as $\star\left(\widetilde{\vartheta}^{a_{1} \cdots a_{p}}\right)=\varepsilon^{a_{1} \cdots a_{n}} \widetilde{e}_{a_{p+1} \cdots a_{n}}$. Here $\widetilde{e}_{a_{i}}(i=p+1, \ldots, n)$ are understood as the down indexed 1-forms $\widetilde{e}_{a_{i}}=o_{a_{i} b} \widetilde{\vartheta}^{b}$ and $\varepsilon^{a_{1} \ldots a_{n}}$ is the total antisymmetric pseudo-tensor.

The variation of the connection 1-form $\widetilde{\omega}^{a b}$ yields $\delta \widetilde{S}_{Q}=\frac{1}{\nsim} \star \star \widetilde{\mathcal{T}}_{a b} \wedge \delta \widetilde{\omega}^{a b}$, where $\star \widetilde{\mathcal{T}}_{a b}:=$ $\frac{1}{2} \star\left(\widetilde{Q}_{a} \wedge \widetilde{e}_{b}\right)=\widetilde{Q}^{c} \wedge \widetilde{\vartheta}^{d} \varepsilon_{c d a b}=\frac{1}{2} \widetilde{Q}^{c}{ }_{\mu \nu} \wedge{\widetilde{e^{d}}}_{\alpha} \varepsilon_{a b c d} \widetilde{\vartheta}^{\mu \nu \alpha}$, here we used the abbreviated notations for the wedge product monomials, $\widetilde{\vartheta}^{\mu \nu \alpha \ldots}=\widetilde{\vartheta}^{\mu} \wedge \widetilde{\vartheta}^{\nu} \wedge \widetilde{\vartheta}^{\alpha} \wedge \ldots$, defined on the $U_{4}$ space, and that $\widetilde{Q}^{a}=\widetilde{D} \widetilde{\vartheta}^{a}=d \widetilde{\vartheta}^{a}+\widetilde{\omega}^{a}{ }_{b} \wedge \widetilde{\vartheta}^{b}$. The variation of the action describing the macroscopic matter sources $\widetilde{S}_{m}$ with respect to the coframe $\vartheta^{a}$, and connection 1 -form $\widetilde{\omega}^{a b} \operatorname{reads} \delta \widetilde{S}_{m}=\int \delta \widetilde{L}_{m}=\int\left(-\star \widetilde{\theta}_{a} \wedge \delta \widetilde{\vartheta}^{a}+\right.$ $\left.\frac{1}{2} \star \widetilde{\Sigma}_{a b} \wedge \delta \widetilde{\omega}^{a b}\right)$, where $\star \widetilde{\theta}_{a}$ is the dual 3-form relating to the canonical energy-momentum tensor, $\widetilde{\theta}_{a}^{\mu}$, by $\star \widetilde{\theta}_{a}=\frac{1}{3 !} \widetilde{\theta}_{a}^{\mu} \varepsilon_{\mu \nu \alpha \beta} \widetilde{\vartheta}^{\nu \alpha \beta}$. and $\star \widetilde{\Sigma}_{a b}=-\star \widetilde{\Sigma}_{b a}$ is the dual 3-form corresponding to the canonical spin tensor, which is identical with the dynamical spin tensor $\widetilde{S}_{a b c}$, namely $\star \widetilde{\Sigma}_{a b}=\widetilde{S}_{a b}^{\mu} \varepsilon_{\mu \nu \alpha \beta} \widetilde{\vartheta}^{\nu \alpha \beta}$. The variation of the total action, $\widetilde{S}=\widetilde{S}_{g}+\widetilde{S}_{m}$, with respect to the $\widetilde{e}_{a}, \widetilde{\omega}^{a b}$ and $\widetilde{\Phi}$ gives the field equations: 1) $\left.\left.\frac{1}{2} \stackrel{\circ}{R}_{c d} \wedge \widetilde{\vartheta}^{c}=æ \widetilde{\theta}_{d}=0,2\right) \star \widetilde{\mathcal{T}}_{a b}=-\frac{1}{2} æ \star \widetilde{\Sigma}_{a b}, 3\right) \frac{\delta \widetilde{L}_{m}}{\delta \widetilde{\Phi}}=0, \quad \frac{\delta \widetilde{L}_{m}}{\delta \widetilde{\Phi}}=0$.The DC-members $\widetilde{D}$ and $\widetilde{\psi}$ can readily be determined as follows: $\widetilde{D}_{a}^{l}=\eta^{l m}<\widetilde{e}_{a}, e_{m}>, \widetilde{\psi}_{l}^{a}=\eta_{l m} \widetilde{\vartheta}^{a}\left(\vartheta^{-1}\right)^{m}$.

\section{The microscopic theory of black hole: Preliminaries}

The theoretical framework in which we shall tackle the problem in quest is the framework of GGP. Exploring a spontaneous breaking of gravitation gauge symmetry at huge energies, the GGP underlies the MTBH which, in turn, has proved to be quite fruitful for ultra-high energy astrophysics. Although the agreement is satisfactory between MTBH and the solar system observational verifications and, moreover, it is consistent with general relativity up to the limit of neutron stars, nevertheless, one of the most remarkable drawback of this theory is the fact that instead of infinite collapse and central singularity, an inevitable end product of the evolution of massive object is the stable SPC, where static observers exist.

For a benefit of the reader, as a guiding principle to make the rest of paper understandable, in this Section we necessarily recount some of the highlights behind of MTBH, which are in use throughout the paper. Needless to say that we will refrain from providing lengthy mathematical details of MTBH. We will not be concerned with the actual details here, but only use it as a backdrop to validate the MTBH with some observational tests. Although some key theoretical ideas were introduced with 
a satisfactory substantiation, we have also attempted to maintain a balance between being overly detailed and overly schematic.

\subsection{The equilibrium superdense proto-matter core}

For brevity reasons, in our setting we proceed in relatively simple way toward first look at some of the associated physics, which is quick to estimate the physical properties of more realistic SPCconfigurations. The equations describing the equilibrium SPC include the gravitational and ID field equations, the hydrostatic equilibrium equation, and the state equation of the spherical-symmetric distribution of baryonic-quark matter in many-phase stratified states specified for each domain. The state equation will be discussed separately step-by-step away from the domain of lower density up to the domain of higher density.

Field equations. The field equations follow at once from the total gauge invariant Lagrangian in terms of Euler-Lagrange variations, respectively on both the $4 \mathrm{D}$ semi-Riemannian space $V_{4}=\widetilde{R}^{3} \oplus \widetilde{R}^{0}$, and the $4 \mathrm{D}$ flat space $M_{4}=R^{3} \oplus R^{0}$. Hereinafter the quantities referring to $V_{4}$ are denoted by wiggles, and are left without wiggles if they correspond to $M_{4}$. We are interested in the case of a 1D sphericalsymmetric gravitational field $\left.\left(a_{0}(r)\right),\left(r \in R^{3}\right)\right)$, in presence of 1D space-like ID-field $(\bar{a}(r))$. In the case at hand, one has the group of motions $S O(3)$ with $2 \mathrm{D}$ space-like orbits $S^{2}$ where the standard coordinates are $\widetilde{\theta}$ and $\widetilde{\varphi}$. The stationary subgroup of $S O(3)$ acts isotropically upon the tangent space at the point of sphere $S^{2}$ of radius $\widetilde{r}$. So, the bundle $p: V_{4} \rightarrow \widetilde{R}^{2}$ has the fiber $S^{2}=p^{-1}(\widetilde{x}), \quad \widetilde{x} \in V_{4}$ with a trivial connection on it, where $\widetilde{R}^{2}$ is the quotient-space $V_{4} / S O(3)$. Considering the equilibrium configurations of degenerate baryonic-quark matter, we assume a spherical-symmetric gravitational field $a_{0}(r)$ in presence of one-dimensional space-like ID-field $\bar{a}$. That is, $a_{(1,1,3)}=a_{(2,2,3)}=a_{(+3)}=$ $\frac{1}{2}\left(-a_{0}+\bar{a}\right), a_{(1,2,3)}=a_{(2,1,3)}=a_{(-3)}=\frac{1}{2}\left(-a_{0}-\bar{a}\right), a_{(\lambda, \mu, 1)}=a_{(\lambda, \mu, 2)}=0, \quad \lambda, \mu=1,2 . ;$ an absence of transversal stresses and the transference of masses in the space $V_{4}: T_{1}^{1}=T_{2}^{2}=T_{3}^{3}=$ $-\widetilde{P}(\widetilde{r}), \quad T_{0}^{0}=-\widetilde{\rho}(\widetilde{r})$, where $T_{\nu}^{\mu}$ is taken to denote the components of energy stress tensor. The equations of gravitation, $x_{0}:=æ a_{0}$, and ID, $x:=æ \bar{a}$, fields can be written in Feynman gauge as follows (Ter-Kazarian, 2014, 2015): $\Delta x_{0}=-\mathfrak{x}^{2}\left\{\frac{1-x_{0}}{\left(1-x_{0}\right)^{2}+x^{2}} \widetilde{\rho}(\widetilde{r})+\frac{1+x_{0}}{\left(1-x_{0}\right)^{2}+x^{2}} \widetilde{P}(\widetilde{r})\right\},\left(\Delta-\lambda_{a}^{-2}\right) x=$ $æ^{2} x\left\{\frac{\widetilde{\rho}(\widetilde{r})}{\left(1-x_{0}\right)^{2}+x^{2}}-\frac{\widetilde{P}(\widetilde{r})}{\left(1-x_{0}\right)^{2}+x^{2}}\right\} \times \theta\left(\lambda_{a}-n^{-1 / 3}\right)$. Reviewing notations $æ$ is the coupling constant relating to the Newton gravitational constant $(G)$ as $æ=8 \pi G / c^{4}, \widetilde{P}(\widetilde{r})$ and $\widetilde{\rho}(\widetilde{r}) \quad\left(\widetilde{r} \in \widetilde{R}^{3}\right)$ are taken to denote the internal pressure and macroscopic density of energy defined in proper frame of reference that is being used, $\widetilde{n}$ is the distorted concentration of particles, $r$ is the radius-vector defined on flat space $R^{3}, \Delta \equiv \partial^{2} / \partial r^{2}, \theta(t)$ is the step function $\theta(t)=\left\{\begin{array}{ll}1 & t \geq 0 \\ 0 & t<0\end{array}\right.$, and $\lambda_{a}$ is the Compton length of the ID-field: $\lambda_{a}=\hbar / m_{a} c \simeq 0.4 \mathrm{fm}$. A diffeomorphism $\widetilde{r}(r): M_{4} \rightarrow V_{4}$ is defined as $r=\widetilde{r}-R_{g} / 4$, where $R_{g}$ is the gravitational radius of distribution of matter, $R_{g}=2 G M / c^{2}=2.95 \times 10^{5} M / M_{\odot} \mathrm{cm}$.

Phase transition of II-type. In case at hand, the aforementioned profound geometrical structures enable an insight to explore a novel aspects expected at distortion of basis vectors $\left(e_{\lambda \alpha}, \bar{e}_{\tau \beta}\right)$ of $\widetilde{M}_{12}$, where $\tan \theta_{( \pm 3)}=æ\left(-a_{0} \pm \bar{a}\right)$. Namely, $\widetilde{e}_{0}=e_{0}\left(1-x_{0}\right)+\bar{e}_{3} x, \quad \widetilde{e}_{3}=e_{3}\left(1+x_{0}\right)-\bar{e}_{03} x, \quad \widetilde{e}_{1}=$ $\frac{1}{2}\left\{\left(\cos \theta_{(+3)}+\cos \theta_{(-3)}\right) e_{1}+\left(\sin \theta_{(+3)}+\sin \theta_{(-3)}\right) e_{2}+\left(\cos \theta_{(+3)}-\cos \theta_{(-3)}\right) \bar{e}_{01}+\left(\sin \theta_{(+3)}-\sin \theta_{(-3)}\right) \bar{e}_{02}\right\}$, $\widetilde{e}_{2}=\frac{1}{2}\left\{\left(\cos \theta_{(+3)}+\cos \theta_{(-3)}\right) e_{2}-\left(\sin \theta_{(+3)}+\sin \theta_{(-3)}\right) e_{1}+\left(\cos \theta_{(+3)}-\cos \theta_{(-3)}\right) \bar{e}_{02}-\left(\sin \theta_{(+3)}-\right.\right.$ $\left.\left.\sin \theta_{(-3)}\right) \bar{e}_{01}\right\}$. This straightforwardly yields the transformations of Poincaré generators of translations, which, in turn, lead to the phase transition of each particle located in the ID-region: $\widetilde{E}=E, \quad \widetilde{P}_{1,2}=$ $P_{1,2} \cos \widetilde{\theta}_{3}, \quad \widetilde{P}_{3}=P_{3}-\tan \widetilde{\theta}_{3} m c, \widetilde{m}=\left|\left(m-\tan \widetilde{\theta}_{3} \frac{P_{3}}{c}\right)^{2}+\sin ^{2} \widetilde{\theta}_{3} \frac{P_{1}^{2}+P_{2}^{2}}{c^{2}}-\tan ^{2} \widetilde{\theta}_{3} \frac{E^{2}}{c^{4}}\right|^{\frac{1}{2}}$, where $E, \vec{P}, m$ and $\widetilde{E}, \widetilde{\vec{P}}, \widetilde{m}$ are, respectively, ordinary and distorted energy, momentum and mass at rest, and $\tan \widetilde{\theta}_{3}=$ $-x, \quad \widetilde{\theta}_{1}=\widetilde{\theta}_{2}=0$. Consequently, a whole matter found in the ID-region of spacetime continuum is undergone phase transition of II-type, while a shift of mass and energy-momentum spectra occurs upwards along the energy scale. The thermodynamics of a resulting matter, so-called proto-matter, is drastically differed from the thermodynamics of strongly compressed ordinary matter. It was in close analogy of well known Nambu-Jona-Lasinio model (Nambu \& Jona-Lasinio, 1961) of elementary particle physics, to generate a fermion mass within the framework of gauge fields due to rearrangement 
of vacuum state. The formation of proto-matter is a main reason for a sharply increase of energy density and internal pressure in the central part of proto-matter configuration, which is proportional to strong gravitational forces of compression. This halts the collapse and the central singularity would not occur. The resulting stable SPC is formed, which consists of the proto-matter core (PC) and the outer layers of ordinary matter. Thus, such phase-transition of matter has smeared out a central singularity of $\mathrm{BH}$ at very strong gravitational fields, replacing it by the equilibrium SPC.

The explicit form of the line element from the outside of configuration $\widetilde{r}>\widetilde{r}_{b}$, where $\widetilde{r}_{b}$ is the boundary of distribution of matter, reads $d s^{2}=\left(1-x_{0}\right)^{2} d \widetilde{t}^{2}-\left(1+x_{0}\right)^{2} d \widetilde{r}^{2}-\widetilde{r}^{2}\left(\sin ^{2} \theta d \varphi^{2}+d \theta^{2}\right)$. Given the state equation, the hydrostatic equilibrium equation can be integrated. While an integration constant is determined from the condition of matching of internal and external metrics. Hence $g_{00}\left(r_{f}\right)=\left(1-\frac{R_{g}}{2 r_{b}}\right)^{2} \exp \left[\int_{0}^{\widetilde{P}} \frac{2 \widetilde{P}}{\widetilde{P}+\widetilde{\rho}}\right]$. Before proceeding further, and to make the reader fully understood, it is worthwhile to discuss in more detail one principle issue in use. Recall that according to the fundamental idea, conceived in the framework of GR, the EH is impenetrable barrier for crossing from inside the $\mathrm{BH}$, because of a singularity arisen at Schwarzschild radius. But this barrier disappears in the framework of MTBH, when a matter, located in ID-region of the spacetime continuum, has undergone phase transition of II-type and, thus, it becomes a proto-matter. To obtain some feeling about this phenomena, note that, a singularity at intersection of proto-matter disk with the event horizon disappears where a massive component of ID-field is not zero, and hence the crossing event horizon from inside of $\mathrm{BH}$ at such conditions is allowed.

\subsection{A research design and methods}

We implement our scheme by considering the equilibrium configurations of non-rotating SPC of the two classes, with spherical-symmetric distribution of matter in many-phase stratified states. A layering of configuration is a consequence of the onset of different regimes in equation of state. The I-class configurations include:

Domain $\rho<\rho_{\text {drip }}=4.3 \times 10^{11} \mathrm{~g} \mathrm{~cm}^{-3}$ - the shell made of cold catalized matter, which is formed after nuclear burning in the density range below neutron drip $\left(\rho_{\text {drip }}\right)$. Below $10^{7} \mathrm{~g} \mathrm{~cm}^{-3}$, the ${ }_{26}^{56} \mathrm{Fe}$ nuclei are dominating. In the inner crust, a Coulomb lattice of heavy nuclei co-exist in $\beta$ - equilibrium with relativistic electrons.

Domain $\rho_{\text {drip }} \leq \rho<4.5 \times 10^{12} \mathrm{~g} \mathrm{~cm}^{-3}$ inner crust-the electrons, nuclei and free neutrons co-exist in the medium.

Above the density $\rho>4.5 \times 10^{12} \mathrm{~g} \mathrm{~cm}^{-3}$ the I-class configurations are thought to be composed of two phases of ideal cold n-p-e gas, which is mixture of neutrons, protons and electrons in complete $\beta$ equilibrium. The first phase state covers the intermediate density -

Domain $4.5 \times 10^{12} \mathrm{~g} \mathrm{~cm}^{-3} \leq \rho<\rho_{d}=2.6 \times 10^{16} \mathrm{~g} \mathrm{~cm}^{-3}$ - which is the regular n-p-e gas in absence of ID. Second phase state is -

Domain $\rho>\rho_{d}$ - the n-p-e proto-matter at short nucleon-nucleon distances $r_{N N} \leq 0.4 \mathrm{fm}$, in presence of ID.

For the II-class SPC configurations, up to the density range $\rho \leq \rho_{f l}=4.1 \times 10^{14} \mathrm{~g} \mathrm{~cm}^{-3}$, to which the $r_{N N} \leq 1.6 \mathrm{fm}$ nucloun-nucleon distances correspond, one has the same domains of I-class configurations. Above the density $\rho_{f l}$, we consider an onset of melting down of hadrons when nuclear matter consequently turns to quark matter. In the domain of $\rho_{f l} \leq \rho<\rho_{a s}=m_{n}(0.25 \mathrm{fm})^{3} \simeq 1.1 \times 10^{17} \mathrm{~g} \mathrm{~cm}^{-3}$, where $m_{n}$ is the neutron mass at rest, $0.25 \mathrm{fm}$ is the string thickness, we consider two phase states of string flip-flop regimes.

Domain $\rho_{f l} \leq \rho<\rho_{d}$, to which the distances $0.4 \mathrm{fm}<r_{N N} \leq 1.6 \mathrm{fm}$ correspond - the regular string flip-flop, when ID is absent. This is a kind of tunneling effect when the strings joining the quarks stretch themselves violating energy conservation and after touching each other they switch on to the other configuration. We are interested in the individual particle approximation (Hartree approximation), where the Hartree potential is almost linearly proportional to the string length. The $Y$ shape string is the most convenient for calculations, because the center of it almost equals to the center of gravity. At very first, we shall study the classical strings. In analogy to the case of ordinary quark matter, one may readily show that in order to have bound state the rising potential should be a scalar. 
Similar to ordinary case a red quark searches for the nearest center and joins with it by a string and so on. One simplifies the calculations by assuming that the centers are uniformly distributed with a particle concentration. We assume that quarks have small ordinary mass $m_{i} \simeq m_{u}=5 \mathrm{MeV}$. Next, we explore a tunneling effect of quantum fluctuations of string, and the negative potential energy caused by such a quantum jump. The basic technique adopted for calculation of transition matrix element $\widetilde{K}$ is the instanton technique (semi-classical treatment). Due to quantum string flip-flop, an attractive interaction between quarks is presented, when during the quantum transition from a state $\psi_{1}$ of energy $\widetilde{E}_{1}$ to another one $\psi_{2}$ of energy $\widetilde{E}_{2}$, the lowering of energy of system occurs. The quark matter acquires $\Delta \widetilde{E}$ correction to the classical string energy, such that the flip-flop energy lowers the energy of quark matter, consequently by lowering the critical density or critical Fermi momentum. The quark matter acquires $\Delta \widetilde{E}$ correction to the classical string energy, such that the flip-flop energy lowers the energy of quark matter, consequently by lowering the critical density or critical Fermi momentum. If one, for example, looks for the string flip-flop transition amplitude of simple system of $q \bar{q} q \bar{q}$ described by the

Hamiltonian $\widetilde{H}$ and invariant action $\widetilde{S}$, then one has $<\mathfrak{!}\left|e^{-\widetilde{H} T}\right| \vdots \dot{\bullet}>=<\int[d \widetilde{\sigma}] e^{-\widetilde{S}}>$, where $T$ is a (imaginary) time interval, $[d \widetilde{\sigma}]$ is the integration over all the possible string motion. The action $\widetilde{S}$ is proportional to the area $\widetilde{A}$ of the surface swept by the strings in the finite region of

ID-region of $V_{4}$. The strings are initially in the $\because$ configuration and finally in the $\mathfrak{l}$ ? configuration. Note that the maximal contribution to the path integral comes from the surface $\sigma_{0}$ of the minimum surface area (instanton). A computation of the transition amplitude is strightforward by summing over all the small vibrations around $\sigma_{0}$. Note that string has a finite thickness $d$, and the width of the area $\Delta \widetilde{A}$ cannot be less than $d$. This cutoff introduces a factor $\exp \left(-a_{0} d r_{N N}\right)$, (where $r_{N N}$ is the distance between two separated centers) in the amplitude $\widetilde{K}$ resulting in the finite-ranged potential. The interaction energy between two centers has a range of order $2 \widetilde{\bar{r}}$ due to overlap of wave functions. A string thickness $d$ can be estimated to be $0.25 \mathrm{fm}$.

Domain $\rho_{d} \leq \rho<\rho_{a s^{-}}$the string flip-flop regime in presence of ID at distances $0.25 \mathrm{fm}<r_{N N} \leq 0.4 \mathrm{fm}$ - a system is made of quark proto-matter in complete $\beta$-equilibrium with rearrangement of string connections joining them.

Domain $\rho>\rho_{a s^{-}}$the system is made of quarks in one beg in complete $\beta$-equilibrium at presence of ID, under the weak interactions and gluons, including the effects of QCD-perturbative interactions. The QCD vacuum has a complicated structure due to the glueon-glueon interactions. The confinement of quarks is a natural feature of the exercising a pressure $B$ on the surface of the local region of the perturbative vacuum to which quarks are confined. This is just the main idea of phenomenological MIT quark bag model, where quarks are assumed to be confined in a bag. Due to the screening of strong forces, the quarks are considered to be free inside the bag and to interact only in the surface region. The surface energy is estimated to be proportional to quark density. The stability of the hadron is ensured by the vacuum pressure $B$ and surface tension. The surface energy is estimated to be proportional to quark density. In most applications, sufficient accuracy is obtained by assuming that all the quarks are almost massless inside a bag. Now, our purpose is to convert this picture to the medium of quark proto-matter. The quark proto-matter is in overall color singlet ground state, which is a noninteracting relativistic Fermi gas found in the ID-region of the spacetime continuum, at $r_{N N} \leq 0.25 \mathrm{fm}$. We consider the quark proto-matter of $u, d$ and $s$ flavors, in complete $\beta$-equilibrium. Now, let discuss the QCD interaction effects in approximation at hand, with extension to quark proto-matter. The first effect is the shift of the vacuum energy per unit volume. The bag constant $B \simeq 55 \mathrm{MeV} / \mathrm{fm}^{3}$ of the MIT bag model must be added to the kinetic energy density. Including the gluon exchange perturbative interactions the energy density of quark proto-matter is then given by the non-interacting Fermi contribution plus bag constant $\widetilde{E}_{0}=\sum_{i} \frac{3}{4} \widetilde{\nu}_{i} \widetilde{P}_{F i} \widetilde{b}_{I}\left(\widetilde{N}, \alpha_{c}\right)+B$, where $\widetilde{P}_{F i}$ is the distorted Fermi momentum of $i$ flavor, $\widetilde{N}$ is the number of flavors present. The $\widetilde{N}$ and running coupling constant $\alpha_{c}$ takes into account the QCD perturbative interactions. The first correction to the free ground state is the ordinary exchange energy corresponding to the second order closed loop diagrams. Next correction is coming up from the sum of different ring diagrams. With equal numbers of quarks of each flavor presented, the modified function $\widetilde{b}_{I}\left(\widetilde{N}, \alpha_{c}\right)$ reads $\widetilde{b}_{I}\left(\widetilde{N}, \alpha_{c}\right)=\left(\widetilde{N}\left[1+\frac{2 \alpha_{c}}{3 \pi}+\frac{\alpha_{c}^{2}}{3 \pi^{2}}\left(\widetilde{N} \ln \frac{\alpha_{c} \widetilde{N}}{\pi}+0.02 \widetilde{N}+6.75\right)\right]\right.$. For numerical calculations it is sufficient to make use of the value $\alpha_{c} \simeq 2.2$ fitting the MIT bag model. 


\subsection{Simulations}

Each configuration is defined by two free parameters of central values of particle concentration $\widetilde{n}(0)$ and ID-field $x(0)$. The interior gravitational potential $x_{0}^{\text {int }}(r)$ should be matched into the exterior one $x_{0}^{\text {ext }}(r)$. Checking it out one introduces the dimensionless sewing parameter $D\left(r_{b}\right)=$ $\left|x_{0}^{i n t}\left(r_{b}\right)-x_{0}^{e x t}\left(r_{b}\right)\right| / x_{0}^{\text {int }}\left(r_{b}\right)$, provided by the subsidiary sewing condition $D\left(r_{b}\right)=0$ imposed at the boundary of configuration. The central value of the gravitational potential $x_{0}(0)$ can be found by reiterating integrations when the sewing condition.

Stability of SPC. In the relativistic case a total mass-energy $M$ of configuration is the extremum in equilibrium for all configurations with the same total number of baryons. While the extrema of $M$ and $N$ occur at the same point in a one-parameter equilibrium sequence, therefore one can extremize $\widetilde{E}=\widetilde{M} c^{2}-\widetilde{m}_{B} N$ on equal footing. Minimizing the energy will give the equilibrium configuration, and the second derivative of $\widetilde{E}$ will give stability information. For a spherical configurations of matter, instantaneously at rest, small radial deviations from equilibrium are governed by a Sturm-Liouville linear eigenvalue equation, with the imposition of suitable boundary conditions on normal modes with time dependence. A necessary and sufficient condition for stability is that the potential energy be positive defined for all initial data of small perturbations, namely, in first order approximation when one does not take into account the rotation and magnetic field, if the square of frequency of normal mode of small perturbations is positive. A relativity tends to destabilize configurations. For the SPC one should note that the total mass $M$ is located in the region of $r \leq \bar{r}, \quad \bar{r}$-is the boundary of distribution of protomatter, thus, the ID-field $x(r) \neq 0$ gives an additional contribution $(\nabla \widetilde{P}-\nabla P)$ to the potential energy of interaction, where $\nabla P$ is the gradient of internal pressure, and the quantities denoted by wiggles refer to SPC while the corresponding quantities of quark-star or neutron star are left without wiggles. The increase of distorted (effective) mass $\widetilde{m}$ of each particle of photometer at sufficiently strong $x(r)>>1$ ID is governed by the rule $|\widetilde{m}| \sim x m>>m$. Since the internal pressure $\widetilde{P}$ and microscopic energy density $\widetilde{\rho}$ of degenerate superdence matter is proportional to the fourth degree of $\propto \widetilde{m}^{4}$, then their increase will be on the order of magnitude $\propto x^{4}$ with respect to the conventional value for neutron star. While the gradient of pressure should be increased as $\partial \widetilde{P} / \partial r \propto x^{3}(\partial x / \partial r)$. On the other hand, one has for the gravitational force $(\widetilde{P}+\widetilde{\rho})\left(g^{00} \partial g_{00} / 2 \partial r\right) \propto-x^{3}\left(\partial x_{0} / \partial r\right)$. From the field equation we find that $x_{0} \simeq x>>1, \quad r \leq \bar{r}$ in central region of SPC, therefore $\widetilde{W} \approx W$ (W is the potential corresponding to neutron star) and, accordingly, this condition yields $\bar{\Gamma}_{1}>1.671$, which is analogous to the case of neutron star. Keeping in mind aforesaid, a numerical integrations (TerKazarian et al., 2007) lead to the $\bar{\Gamma}_{1} \approx 2.216$ for the $\mathrm{SPC}_{I}$, while in the case of $\mathrm{SPC}_{I I}$ we obtain $\bar{\Gamma}_{1} \approx 2.4$, which clearly proves that the condition for stability of SPC is satisfied.

\subsection{Results}

The simulations confirm in brief the following scenario. The energy density and internal pressure have sharply increased in PC proportional to gravitational forces of compression in about 20-25 order of magnitude with respect to corresponding central values of neutron star. This counteracts the collapse and equilibrium holds even for the masses $\sim 10^{10} M_{\odot}$. The stable SPCs are formed, with radial distributions of density, internal pressure and a number of integral characteristics. Thereby the stable equilibrium holds for outward layers too. The SPC accommodates the highest energy scale up to hundreds $\mathrm{ZeV}$ in central proto-matter core. Without loss of generality, the typical features of SPC-configurations are summarised in the Fig. 2. The special units in use denote $P_{O V}=6.469 \times$ $10^{36} \mathrm{erg} \mathrm{cm}^{-3}, \quad \rho_{O V}=7.195 \times 10^{15} \mathrm{~g} \mathrm{~cm}^{-3}$ and $r_{O V}=13.68 \mathrm{~km}$. Moreover, above nuclear density, the SPC always resides inside the event horizon, therefore it could be observed only in presence of accreting matter in its close vicinities. This important feature can be seen even without addressing the exact theory. Actually, setting aside for the moment the details of superdense proto-matter physics, it is clear that the metric will necessarily be matched into Einstein's gravitation metric at weak gravitation limit on large distances far from the SPC of masses $\sim\left(10^{8}\right.$ to $\left.10^{9}\right) \mathrm{M}_{\odot}$ and respectively of small sizes $\sim\left(10^{8}\right.$ to $\left.10^{10}\right) \mathrm{km}$. The latter suggests a presence of the coordinate singularity at $g_{00}=0$. In contrast to Schwarzschild pseudo-singularity, in microscopic theory a true physical singularity occurred at the EH sphere of the radius $r=R_{g} / 2$ such that the SPC is located inside the $\mathrm{EH}$, where $R_{g} \simeq 2.95 \cdot 10^{13} \mathrm{M}_{8} \mathrm{~cm}$ 


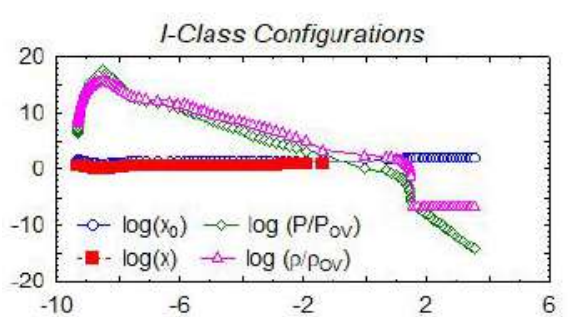

(a) $\log \left(\right.$ r $/ r_{\circ}$ ) $)$

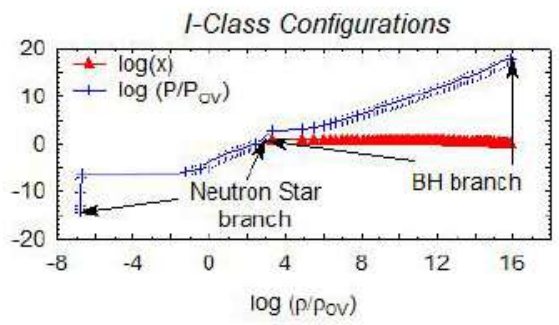

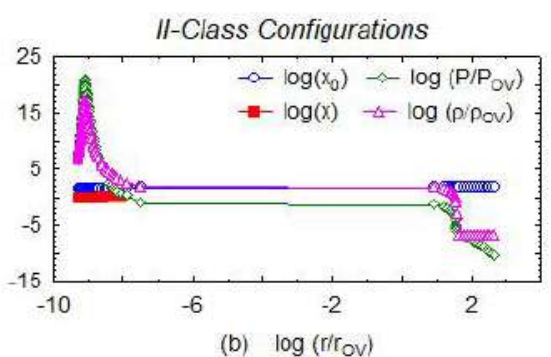

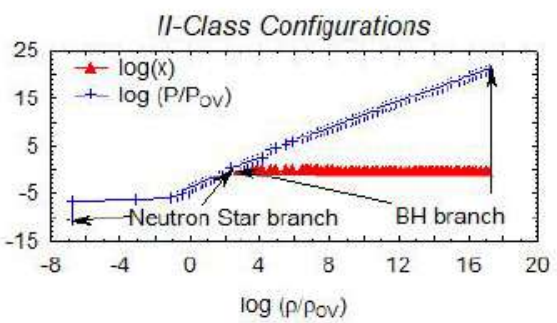

Figure 2. (a) The radial profiles of the pressure, the density, the dimensionless gravitational $\left(x_{0}\right)$ - and ID $(x)$ - potentials, and (b) the state equation, of the SPC of mass $\sim 1.49 \times 10^{5} M_{\odot}$.

is the gravitational radius and $M_{8}=M / 10^{8} M_{\odot}$. The metric component $g_{00}$ is equal zero on the $\mathrm{EH}$ where the curvature invariants are singular. The external physics of accretion onto the SPC in first half of its lifetime is identical to the processes in phenomenological BH models. In other words, there is no observable difference between the gravitational field of SPC and Schwarzschild BH but only one feature as follows. To make the reader fully understood, it is worthwhile before proceeding further to discuss in more detail one principle issue in use. Recall that according to the fundamental idea, conceived in the framework of GR, the EH is impenetrable barrier for crossing from inside the $\mathrm{BH}$, because of a singularity arisen at Schwarzschild radius. But this barrier disappears in the framework of MTBH, when a matter, located in ID-region of the spacetime continuum, has undergone phase transition of II-type and, thus, it becomes a proto-matter. To obtain some feeling about this phenomena, note that (according to the field equations, a singularity at intersection of proto-matter disk with the event horizon disappears where a massive component of ID-field is not zero, and hence the crossing event horizon from inside of $\mathrm{BH}$ at such conditions is allowed.

A crucial difference in the model context between the phenomenological and microscopic black holes comes in when one looks for the spontaneous breaking of gravitation gauge symmetry at huge energies, and thereof making room for growth and merging behavior of black holes. We argue that the black hole seeds might grow driven by the accretion of outside matter when they were getting most of their masses. An infalling matter with time forms a thin proto-matter disk around the proto-matter core tapering off faster at reaching out the event horizon. The thickness of proto-matter disk at the edge of event horizon is of linear size $d$. Whereas a metric singularity inevitably disappears and the $\mathrm{ZeV}$-neutrinos produced via simple or modified URCA processes in deep layers of SPC and protomatter disk may escape from event horizon to outside world through a thin belt area $S=2 \pi R_{g} d$, even after the strong neutrino trapping. The neutrinos are collimated in very small opening angle $\theta_{\nu} \simeq \varepsilon_{d}=\frac{d}{2 R_{g}} \ll 1$. To emphasize the distinction between phenomenological and microscopic black hole models, we present their schematic plots in Fig. 3, to guide the eye.

\section{Growth of accreting supermassive black hole seeds and neutrino radiation}

We further expose the assertions made in the framework of MTBH, to show that the seed black hole might grow up driven by the accretion of outside matter when it was getting most of its mass. 

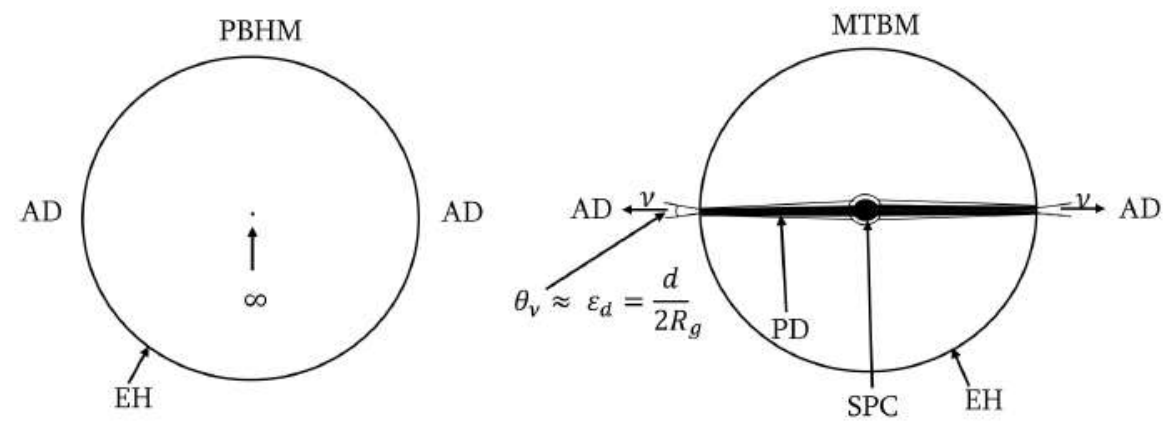

Figure 3. Left panel: Phenomenological model of non-spinning black hole. The meaningless singularity occurs at the center inside the black hole. Right panel: Microscopic model of non-spinning black hole, with the central stable SPC. An infalling matter with the time forms PD around the SPC. In final stage of growth, a PD has reached out the edge of the event horizon. Whereas a metric singularity inevitably disappears and UHE neutrinos may escape from event horizon to outside world through vista - a thin belt area $S=2 \pi R_{g} d$ - with opening angle $\theta_{\nu}$. Accepted notations: EH=Event Horizon, $\mathrm{AD}=$ Accretion Disk, $\mathrm{SPC}=$ Superdense Proto-matter Core, $\mathrm{PD}=$ Proto-matter Disk.

An infalling matter with time formes the proto-matter disk around the proto-matter core tapering off faster at reaching out the thin edge of the event horizon. At this, metric singularity inevitably disappears (see appendices) and the neutrinos may escape through vista to outside world, even after the neutrino trapping. We study the growth of proto-matter disk and derive the intermediate mass and initial redshift of seed black hole, as well as examine luminosities, neutrino surfaces for the disk. We review the mass assembly history of 377 plausible accreting supermassive black hole seeds. After the numerous reiterating integrations of the state equations of SPC-configurations, we compute their intermediate seed masses, $M_{B H}^{\text {Seed }}$, neutrino pre-radiation times (PRTs), initial redshifts, $z^{\text {Seed }}$ and UHE-neutrino fluxes produced in the medium of the SPC via simple (quark and pionic reactions) or modified URCA processes, even after the neutrino trapping. The trapping is due to the fact that as the neutrinos are formed in proto-matter core at super-high densities they experience greater difficulty escaping from the proto-matter core before being dragged along with the matter, namely the neutrinos are trapped comove with matter. The part of neutrinos annihilate to produce, further, the secondary particles of expected ultra-high energies. In this model, of course, a key open question is to enlighten the mechanisms that trigger the activity, and how a large amount of matter can be steadily funneled to the central regions to fuel this activity. In high luminosity AGNs the large-scale internal gravitational instabilities drive gas towards the nucleus that trigger big starbursts, and the coeval compact cluster just formed. It seemed they have some connection to the nuclear fueling through mass loss of young stars as well their tidal disruption, and supernovae. Note that we regard the UHECR particles as a signature of existence of superdence protomatter sources in the universe. Since neutrino events are expected to be of sufficient intensity, our estimates can be used to guide investigations of neutrino detectors for the distant future.

\subsection{Growth of black hole seed in mass}

In the framework of MTBH, a growth behavior of IMBH-candidates widely based on the premises of runaway core collapse scenario. Matter pulled toward the seed BH (proto-matter core) loses angular momentum through viscous or turbulent processes in an intrinsic accretion disk. Within such a disc, friction would cause angular momentum to be transported outward, allowing matter to fall further inward, thus releasing potential energy and increasing the temperature of the proto-matter. One of the key objectives is then the increase of mass $M^{\text {seed }}$ and gravitational radius $R_{g}^{\text {seed }}$ of the seed $\mathrm{BH}$ at accretion of outside matter. Simultaneously with an increase of seed mass, an infalling matter formes intrinsic proto-matter disk around grown up proto-matter core tapering off faster at reaching out the thin edge of EH. The key objectives of the MTBH framework is then an increase of the mass, $M_{B H}^{S e e d}$, and gravitational radius, $R_{g}^{\text {Seed }}$, of the seed black hole, $\mathrm{BH}^{\text {Seed }}$, at accretion of outside matter. Thereby 
Ambartsumian's vision and further insight to key puzzles of ultra-high energy astrophysics

an infalling matter formes proto-matter disk around proto-matter core tapering off faster at reaching out the thin edge of event horizon. So, a practical measure of growth $\mathrm{BH}^{\text {Seed }} \rightarrow \mathrm{BH}$ may most usefully be the increase of gravitational radius or mass of black hole $\Delta R_{g}=R_{g}^{B H}-R_{g}^{S e e d}=\frac{2 G}{c^{2}} M_{d}=\frac{2 G}{c^{2}} \rho_{d} V_{d}$, and that $\Delta M_{B H}=M_{B H}-M_{B H}^{\text {Seed }}=M_{B H}^{S e e d} \frac{\Delta R_{g}}{R_{g}^{S e e d}}$, where $M_{d}, \rho_{d}$ and $V_{d}$ respectively are the total mass, density and volume of proto-matter disk. At the value $\hat{R}_{g}^{B H}$ of gravitational radius, when protomatter disk has finally reached the event horizon of grown-up supermassive black hole, the volume $\hat{V}_{d}$, at $R_{d} \ll \hat{R}_{g}^{B H}$, is $\hat{V}_{d} \simeq \frac{\sqrt{2} \pi}{3} R_{d}\left(\hat{R}_{g}^{B H}\right)^{2}$. From the first line, we obtain $\hat{R}_{g}^{B H}=k\left(1 \pm \sqrt{1-\frac{2}{k} R_{g}^{S e e d}}\right)$, where $2 / k=8.73[\mathrm{~km}] R_{d} \rho_{d} / M_{\odot}$, which is valid at $\frac{2}{k} R_{g}^{\text {Seed }} \leq 1$, namely $\frac{R_{\odot}}{R_{d}} \geq 2.09 \frac{[\mathrm{km}]}{R_{\odot}} \frac{\rho_{d}}{\bar{\rho} \odot} \frac{R_{g}^{\text {Seed }}}{R_{\odot}}$. For the values $\rho_{d}=2.6 \times 10^{16}[\mathrm{~g} \mathrm{~cm}]^{-3}$ (see below) and $R_{g}^{\text {Seed }} \simeq 2.95[\mathrm{~km}]\left(10^{3}\right.$ to $10^{6}$ ), the inequality is reduced to $\frac{R_{\odot}}{R_{d}} \geq 2.34 \times 10^{8}\left(1\right.$ to $\left.10^{3}\right)$ or $\frac{[\mathrm{cm}]}{R_{d}} \geq 0.34\left(10^{-2}\right.$ to 10$)$. This condition always satisfied, because for considered 377 black holes, with the masses $M_{B H} / M_{\odot} \simeq 1.1 \times 10^{6}$ to $1.3 \times 10^{10}$, we approximately have $\frac{R_{d}}{r_{O V}} \simeq 10^{-10}$ to $10^{-7}$. Then the mass of seed black hole reads $\frac{M_{B H}^{S e d}}{M_{\odot}} \simeq \frac{M_{B H}}{M_{\odot}}\left(1-1.68 \times 10^{-6} \frac{R_{d}}{[\mathrm{~cm}]} \frac{M_{B H}}{M_{\odot}}\right)$.

\subsection{The neutrino pre-radiation time}

The PRT is referred to as a lapse of time, $T_{B H}$, from the birth of $\mathrm{BH}$ till neutrino radiation - the earlier part of the lifetime. A typical growth rate for a $\mathrm{BH}$ is then given by the time required to reach the final mass, $M$, and gravitational radius, $\hat{R}_{g}$, when proto-matter disk has finally reached the EH. In this framework we have introduced a notion of the pre-radiation time (PRT) of black hole which is referred to as a lapse of time $T_{B H}$ from the birth of black hole till neutrino radiation - provision for the earlier half of the lifetime of black hole. The PRT-scale can be written (TerKazarian, 2014, 2015) $T_{B H}=\frac{M_{d}}{\dot{M}}$, where $\dot{M}$ is the accretion rate. For given luminosity $L$, reads $T_{B H} \simeq 2.64\left(\frac{\epsilon}{0.1}\right) \frac{R_{d}}{[\mathrm{~cm}]}\left(\frac{M^{\text {seed }}}{M_{\odot}}\right)^{2} \frac{10^{35} \mathrm{~W}}{L}[\mathrm{yr}]$, To give more credit to this view, next we would like to infer an analytical expression for the PRT-scale, $T_{B H}$. The order of magnitudes of the accretion rates can be derived if we assume that there is no shortage of the fuel around the BH. Actually, the BHs are fed by the accretion of gas in a process in which a small fraction of the energy of the accreted gas is released in the form of radiation of intensity $L$. The stars are sufficient to fuel some low luminosity dwarf nuclei: at high densities stellar collisions replenish the central density, and the nuclei can reach higher luminosities. If these conditions are fulfilled, the growth of massive $\mathrm{BH}$ can then be accretiondominated. Thereby the mass accretion rate should be $\dot{M} \equiv \frac{d M}{d t}=\frac{L}{\epsilon c^{2}}$, where $\epsilon$ is the accretion efficiency to transform the gravitational energy into radiation. According to the canonical Bondi accretion rate, the luminosity has increased as $L \propto \dot{M} \propto M^{2}$. The Bondi rate should in general be considered an upper limit on the accretion rate, because it assumes free-fall of gas from the Bondi radius $R_{B}=G M / a_{s}^{2}$, where $a_{s}$ is the sound speed of the gas. At some point, the BH growth seemed to slow down when approaching to quasar phase, for which the gas maximum rate accretion occurs nearly at the Eddington limit, and radiate at Eddington luminosity, $L_{E d d}=\frac{4 \pi G M c}{k}=1.3 \times 10^{38}\left(\frac{M}{M_{\odot}}\right)\left[\mathrm{erg} \mathrm{s}^{-1}\right]$ (above which the radiation pressure prevents the material to fall in), where $k=\sigma_{T} / m_{p}$ is the opacity. So, the $\dot{M}$ is expected to be limited by Eddington accretion rate $\epsilon c^{2} \dot{M} \leq L_{E d d}$. In the same time, it should be emphasized that the possibility of super-Eddington accretion has been also explored theoretically by many authors. A basic reason why this may be feasible is the photon trapping effect on small scales near the BH. That is, in a spherically symmetric accretion flow at a rate much greater than Eddington accretion, the emergent radiation flux is reduced by photon trapping in the optically-thick accreting matter. Such an effect operates when the radial gas in flow speed is faster than the outward photon diffusion speed. The photon trapping effect becomes physically relevant when, so-called, the "trapping radius" $R_{t r}=(k / 4 \pi c) \dot{M}$ is outside $R_{g}$. Note that the Bondi radius is generally much larger than the trapping radius. This idea dates back to the works by Begelman, who constructed in 1978 a global spherical accretion solution for ionized gas at super-Eddington value. The above scenarios holds, however, only in spherical symmetry, and ignores the question of the stability of the flow. The photon-trapping effect has more recently been incorporated into accretion disk models including direct radiation hydrodynamical simulations. These simulations find self-consistent super-Eddington accretion on small scales with lower values of the radiative efficiency below $10 \%$, but are numerically 
limited to model only short durations and small scales. The efficient growth phase can stably exist unless the outward momentum $L / c$ dominates the inward ram pressure of the rapidly accreting gas. In summary, high accretion rates exceeding the Eddington value are possible but produce intense radiation flux toward the polar directions. These results, however, are valid only as long as a sufficient amount of gas at rates of $\dot{M} \gg \dot{M}_{E d d}$ is supplied from larger scales without being impeded by the strong radiation feedback.

In what follows, for simplicity reasons, the mass supply rate from large scales $\sim \dot{M}_{E d d}$ (precisely tracked as a BH grows by orders of magnitude in mass) is of particular interest to us. Then the Salpeter characteristic time-scale becomes as long as $T_{s}=\epsilon t_{E d d}=\epsilon \frac{k c}{4 \pi G}=\frac{M}{\dot{M}_{E d d}}=\left(\frac{d t}{d \log (M)}\right)_{E d d}=\frac{M \epsilon c^{2}}{L_{E d d}}=$ $\left(\frac{\epsilon}{0.1}\right) 4.5 \times 10^{7}[\mathrm{yr}]$. Thence $M\left(\frac{t}{[\mathrm{yr}]}\right)<M(0) \exp \left(\frac{t}{T_{s}}\right)=M(0) \times 10^{\frac{t \log e}{T_{s}}}=M(0) \times 10^{\frac{0.434 t}{T_{s}}}$, so that the characteristic minimum time, $t_{m i n}$, which takes at least $\mathrm{BH}$ of mass $M(0)$ to grow to mass, $M$, at the Eddington rate should be $t>t_{\min } \equiv \frac{T_{s}}{0.434} \log \left(\frac{M}{M(0)}\right)[\mathrm{yr}]=1.037 \times 10^{8} \log \left(\frac{M}{M(0)}\right)[\mathrm{yr}]$, where the value of efficiency is taken $\epsilon \simeq 0.1$ for high redshift quasars. For a seed mass, say, $M(0) \simeq 10^{5} M_{\odot}$, the accretion of mass at the Eddington rate causes a BH mass to increase in time $t_{\min }=1.037 \times 10^{8} \times 4[\mathrm{yr}]=$ $4.148 \times 10^{8}[\mathrm{yr}]$, to $\simeq 10^{9} M_{\odot}$. This brings one back in time to an epoch when the Universe was very young and galaxies in their infancy. For example, the observation of luminous quasars well in excess of $\simeq 10^{47}\left[\mathrm{erg} \mathrm{s}^{-1}\right]$, at $z \simeq 6$, implies that the first SMBHs with masses $\sim 10^{9} M_{\odot}$ must have formed already in place when the Universe is only $1[\mathrm{Gyr}]$ old.

The thickness of proto-matter disk at the edge of event horizon is of linear size $d$. Whereas a metric singularity inevitably disappears and the $\mathrm{ZeV}$-neutrinos produced via simple or modified URCA processes in deep layers of SPC and proto-matter disk may escape from event horizon to outside world through a thin belt area $S=2 \pi R_{g} d$, even after the strong neutrino trapping. The neutrinos are collimated in very small opening angle $\theta_{\nu} \simeq \varepsilon_{d}=\frac{d}{2 R_{g}} \ll 1$. Note that the spherical accretion onto black hole, in general, is not necessarily an efficient mechanism for converting restmass energy into radiation. Accretion onto black hole may be far from spherical accretion, because the accreted gas possesses angular momentum. In this case, the gas will be thrown into circular orbits about the black hole when centrifugal forces will become significant before the gas plunges through the event horizon. Assuming a typical mass-energy conversion efficiency of about $\epsilon \sim 10 \%$, in approximation $R_{d} \ll R_{g}$, the resulting relationship of typical PRT versus bolometric luminosity becomes $T_{B H} \simeq 0.32 \frac{R_{d}}{r_{O V}}\left(\frac{M_{B H}}{M_{\odot}}\right)^{2} \frac{10^{39} \mathrm{~W}}{L_{b o l}}[\mathrm{yr}]$.

\subsection{Redshift of seed black hole}

To follow the history of the seed BH to the present time in the expanding Universe of a general recession of distant galaxies away from us in all directions, the radiation density at the present epoch can be neglected in comparison with the matter density in the Universe. So, the expansion rate of the Universe depends on the matter density, $\rho$, the cosmological constant, $\Lambda$, and the curvature, $k$, of the space. Interpreting the redshift as a cosmological Doppler effect, and that the Hubble law could most easily be understood in terms of expansion of the universe, we are interested in the purely academic question of principle, to ask what could be the initial redshift, $z^{\text {Seed }}$, of seed black hole if the mass, the luminosity and the redshift, $z$, of black hole at present time are known. To follow the history of seed black hole to the present time, let place ourselves at the origin of coordinates $r=0$ (according to the Cosmological Principle, this is mere convention) and consider a light traveling to us along the $-r$ direction, with angular variables fixed. If the light has left a seed black hole, located at $r_{s}, \theta_{s}, \varphi_{s}$, at time $t_{s}$, and it has to reach us at a time $t_{0}$, then a power series for the redshift as a function of the time of flight is $z^{\text {Seed }}=H_{0}\left(t_{0}-t_{s}\right)+\cdots$, where $t_{0}$ is the present moment, $H_{0}$ is the Hubble's constant. Similar expression $z=H_{0}\left(t_{0}-t_{1}\right)+\cdots$, can be written for the current black hole, located at $r_{1}, \theta_{1}, \varphi_{1}$, at time $t_{1}$, where $t_{1}=t_{s}+T_{B H}$, as seed black hole is a object at early times. Hence, in the first-order approximation by Hubble's constant, we may obtain the following relation between the redshifts of seed and present black holes: $z^{\text {Seed }} \simeq z+H_{0} T_{B H}$. This relation is in agreement with the scenario of a general recession of distant galaxies away from us in all directions, the furthest naturally being those moving fastest. This relation, incorporating with the value $H_{0}=70[\mathrm{~km}] /[\mathrm{s} \mathrm{Mpc}]$ favored today yield $z^{\text {Seed }} \simeq z+2.292 \times 10^{28} \frac{R_{d}}{r_{O V}}\left(\frac{M_{B H}}{M_{\odot}}\right)^{2} \frac{W}{L_{b o l}}$. 
The general relation between the PRT-scale and the redshifts of BH and its seed is written (TerKazarian, 2014, 2015): $H_{0} T_{B H}=\int_{z}^{z^{\text {seed }}} \frac{d z^{\prime}}{\left(1+z^{\prime}\right) \sqrt{\Omega_{M}\left(1+z^{\prime}\right)^{3}+\Omega_{K}\left(1+z^{\prime}\right)^{2}+\Omega_{\Lambda}}}$, where $\Omega_{\Lambda}=\frac{\Lambda}{3 H_{0}^{2}}, \Omega_{K}=\frac{-k}{a_{0}^{2} H_{0}^{2}}$, $\Omega_{M}=\frac{\rho}{\rho_{\text {crit }}}$, and the critical density is $\rho_{\text {crit }}=\frac{3 H^{2}}{8 \pi G}$. There are only two independent contributions to the energy density $\Omega_{M}+\Omega_{\Lambda}+\Omega_{K}=1$. The expansion rate (Hubble's parameter) of the Universe at any epoch at redshift less than about 1000 can be related to the one at the present epoch by $H(z)=H_{0} E(z)$, where $E(z) \equiv \sqrt{\left[\Omega_{M}(1+z)^{3}+\Omega_{K}(1+z)^{2}+\Omega_{\Lambda}\right]}$. Let the proper time, $t$, be the temporal measure. This is a convenient time measure because it is the proper time of comoving observers. The lookback time is the time difference between the present epoch, $t_{0}$, and the time of an event that happened at the redshift, $z$. From the definitions of Hubble's parameter and redshift it follows that $H=\frac{d}{d t} \log \left(\frac{R(t)}{R_{0}}\right)=\frac{d}{d t} \ln \left(\frac{1}{1+z}\right)=\frac{-1}{1+z} \frac{d z}{d t}$, where $R$ is called the scale factor of the Universe, and increases as the Universe expands in a manner that depends upon the cosmological model selected. Hence, the lookback time from the present, as a function of the time of flight, reads $t_{0}-t_{1}\left(z_{1}\right)=H_{0}^{-1} \times \int_{0}^{z_{1}} \frac{d z^{\prime}}{\left(1+z^{\prime}\right) \sqrt{\Omega_{M}\left(1+z^{\prime}\right)^{3}+\Omega_{K}\left(1+z^{\prime}\right)^{2}+\Omega_{\Lambda}}}$. Relating the PRT-scale, $T_{B H}$, to the redshifts of $\mathrm{BH}, z$, and its seed, $z^{\text {seed }}$, let we place ourselves at the origin of coordinates, $r=0$, (according to the Cosmological Principle, this is mere convention). Consider a light traveling to us along the $-r$ direction, with angular variables fixed. If the light has left a seed $\mathrm{BH}$, located at $r_{s}, \theta_{s}, \varphi_{s}$, at time $t_{s}$, that happened at the redshift $z^{\text {seed }}$, and it has to reach us at present epoch $t_{0}$, at the redshift $z=0$, then from the definition of the lookback time, it follows that $H_{0}\left(t_{0}-t_{s}\left(z^{\text {seed }}\right)\right)=$ $\int_{0}^{z^{s e e d}} \frac{d z^{\prime}}{\left(1+z^{\prime}\right) \sqrt{\Omega_{M}\left(1+z^{\prime}\right)^{3}+\Omega_{K}\left(1+z^{\prime}\right)^{2}+\Omega_{\Lambda}}}$. Similar expression can be written for the current BH, located at $r_{1}, \theta_{1}, \varphi_{1}$, at time $t_{1}$, with redshift $z: H_{0}\left(t_{0}-t_{1}(z)\right)=\int_{0}^{z} \frac{d z^{\prime}}{\left(1+z^{\prime}\right) \sqrt{\Omega_{M}\left(1+z^{\prime}\right)^{3}+\Omega_{K}\left(1+z^{\prime}\right)^{2}+\Omega_{\Lambda}}}$. Taking into account that $t_{1}=t_{s}+T_{B H}$, we arrived to the general relation between the PRT-scale and the redshifts of $\mathrm{BH}$ and its seed. As a supplement to this relation, we may derive $z_{s}$ as a function of the quantities $z$ and $T_{B H}$. According to the definitions of Hubble's parameter, we may write down $z+1=e^{H(z)\left(t_{0}-t_{1}\right)}, \quad$ for the $\mathrm{BH}, z_{s}+1=e^{H\left(z_{s}\right)\left(t_{0}-t_{s}\right)}$, for the seed BH. Setting $H(z) \simeq H\left(z_{s}\right)$, and taking into account that $t_{1}=t_{s}+T_{B H}$, we obtain $\frac{z+1}{z_{s}+1}=e^{-H(z)\left(t_{1}-t_{s}\right)}=e^{-H(z) T_{B H}}$. Hence, the function $z_{s}\left(z, T_{B H}\right)$ reads $z_{s}=(z+1) e^{H(z) T_{B H}}-1$.

High redshifts: At large redshifts $z>>\Omega_{M}, \Omega_{\Lambda}$, only third power of $z$ in the square root becomes important, and thus we find $T_{B H} \simeq \int_{z}^{z^{\text {seed }}} \frac{d z}{H_{0} \sqrt{\Omega_{M}}(1+z)^{5 / 2}} \simeq \frac{2}{3 H_{0} \sqrt{\Omega_{M}}}\left[\frac{1}{(1+z)^{3 / 2}}-\frac{1}{\left(1+z^{\text {seed }}\right)^{3 / 2}}\right]$. Thus, in time to an epoch when the Universe was very young and galaxies in their infancy, the redshift of seed $\mathrm{BH}$ reads $z^{\text {seed }} \simeq\left[\frac{1}{(1+z)^{3 / 2}}-\frac{3 \sqrt{\Omega_{M}} H_{0} T_{B H}}{2}\right]^{-2 / 3}-1$. Hence $z^{\text {seed }} \simeq\left[\frac{1}{(1+z)^{3 / 2}}-\frac{3 \sqrt{\Omega_{M}}}{2}(18.25 \pm 0.351) \times\right.$ $\left.\left(\frac{\epsilon}{0.1}\right) \frac{R_{d}}{[\mathrm{~cm}]}\left(\frac{M^{\text {seed }}}{M_{\odot}}\right)^{2} \frac{10^{24} W}{L}\right]^{-2 / 3}-1$.

Low redshifts: For low redshifts $z<<1$, as alluding to above, in the first-order approximation by the $z$, we obtain $z^{\text {seed }} \simeq z+H_{0} T_{B H}$. Hence $z^{\text {seed }} \simeq z+(18.25 \pm 0.351)\left(\frac{\epsilon}{0.1}\right) \frac{R_{d}}{[\mathrm{~cm}]}\left(\frac{M^{\text {seed }}}{M_{\odot}}\right)^{2} \frac{10^{24} W}{L}$.

\subsection{UHE cosmic-ray particles}

The galactic sources like supernova remnants (SNRs) or micro-quasars are thought to accelerate particles at least up to energies of $3 \times 10^{15} \mathrm{eV}$. The ultra-high energy cosmic-ray (UHECR) particles with even higher energies have since been detected. Although more than five decades passed since the famous first detection of cosmic rays with huge energies exceeding $1.0 \times 10^{20} \mathrm{eV}$ by the Volcano Ranch group led by John Linsley (Linsley, 1963), nevertheless, the solution to this outstanding puzzle had not been achieved yet, and this principle problem was ever since much the same as now. At present, about 20 events above $10^{20} \mathrm{eV}$ have been reported worldwide by experiments such as the High Resolution Fly's Eye, AGASA, Fly's Eye, Haverah Park, Yakutsk, and Volcano Ranch, etc.. The cosmic-ray events with the highest energies so far detected have energies of $2 \times 10^{11} \mathrm{GeV}$ and $3 \times 10^{11} \mathrm{GeV}$. These energies $10^{7}$ times higher than the most energetic man-made accelerator, the LHC at CERN. These highest energies are believed to be reached in extragalactic sources like AGNs or gamma-ray bursts (GRBs). During propagation of such energetic particles through the universe, the threshold for pion photoproduction on the microwave background is $\sim 2 \times 10^{10} \mathrm{GeV}$, and at $\sim 3 \times 10^{11} \mathrm{GeV}$ the energy-loss distance is about $20 \mathrm{Mpc}$. Propagation of cosmic-rays over substantially larger distances gives rise to a cutoff in the spectrum at $\sim 10^{11} \mathrm{GeV}$, so-called GZK-cutoff (Greisen-Zatsepin-Kuzmin) as was first shown by these 


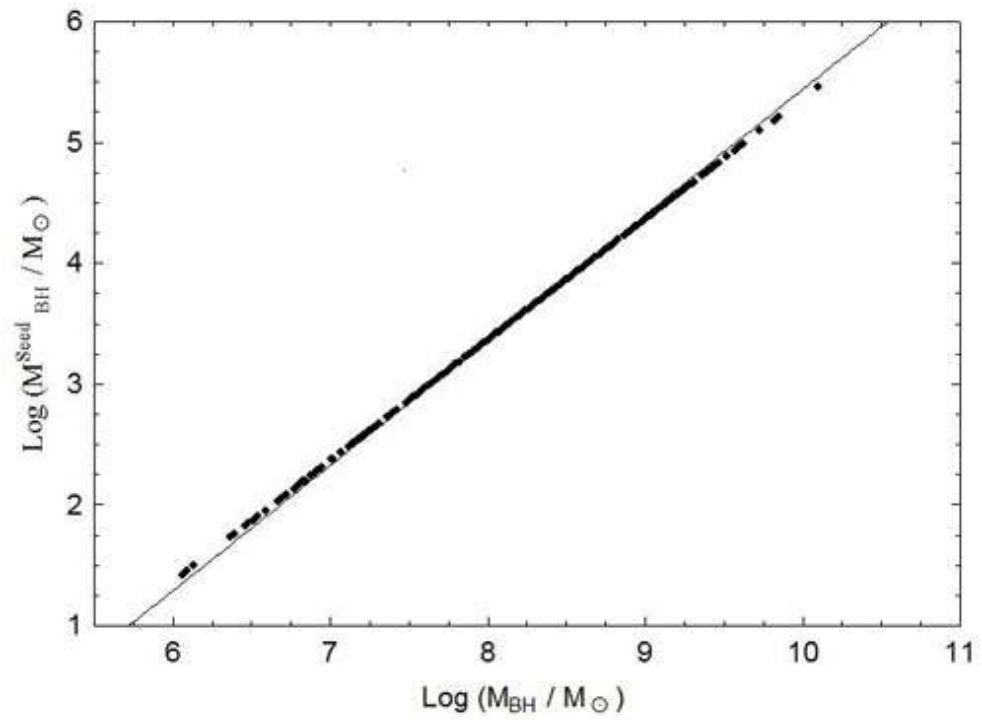

Figure 4. The $M_{B H}^{\text {Seed }} / M_{\odot}-M_{B H} / M_{\odot}$ relation on logarithmic scales of 337 black holes. The solid line is the best fits to data of samples.

authors. The recent confirmation of GZK suppression in the cosmic-ray energy spectrum indicates that the cosmic-rays with energies above the GZK cutoff, $E_{G Z K} \sim 40 \mathrm{EeV}$, mostly come from relatively close (within the GZK radius, $r_{G Z K} \sim 100 \mathrm{Mpc}$ ) extragalactic sources. However, despite the detailed measurements of the cosmic-ray spectrum, the identification of the sources of the cosmic-ray particles is still an open question as they are deflected in the Galactic and extragalactic magnetic fields and hence have lost all information about their origin when reaching Earth. Only at the highest energies beyond $\sim 10^{19.6} \mathrm{GeV}$ cosmic-ray particles may retain enough directional information to locate their sources. The latter must be powerful enough to sustain the energy density in extragalactic cosmic-rays of about $3 \times 10^{-19} \mathrm{erg} \mathrm{cm}^{-3}$ which is equivalent to $\sim 8 \times 10^{44} \mathrm{erg}^{\mathrm{Mpc}}{ }^{-3} \mathrm{yr}^{-1}$. Though it hasn't been possible up to now to identify the sources of Galactic or extragalactic cosmic-rays, general considerations allow to limit potential source classes. Eventually, the neutrinos will serve as unique astronomical messengers, and that they will significantly enhance and extend our knowledge on Galactic and extragalactic sources of the UHE universe. Indeed, except for oscillations induced by transit in a vacuum Higgs field, neutrinos can penetrate cosmological distances and their trajectories are not deflected by magnetic fields as they are neutral, providing powerful probes of high energy astrophysics in ways which no other particle can. Moreover, the flavor composition of neutrinos originating at astrophysical sources can serve as a probe of new physics in the electroweak sector. Therefore, an appealing possibility among the various hypotheses of the origin of UHECR is so-called Z-burst scenario. This suggests that if ZeV astrophysical neutrino beam is sufficiently strong, it can produce a large fraction of observed UHECR particles within $100 \mathrm{Mpc}$ by hitting local light relic neutrinos clustered in dark halos and form UHECR through the hadronic Z (s-channel production) and W- bosons (t-channel production) decays by weak interactions. The discovery of UHE neutrino sources would also clarify the production mechanism of the $\mathrm{GeV}-\mathrm{TeV}$ gamma-rays observed on Earth as $\mathrm{TeV}$ photons are also produced in the up-scattering of photons in reactions with accelerated electrons (inverse-Compton scattering). The direct link between $\mathrm{TeV}$ gamma-ray photons and neutrinos through the charged and neutral pion production, which is well known from particle physics, allows for a quite robust prediction of the expected neutrino fluxes provided that the sources are transparent and the observed gamma rays originate from pion decay. The weakest link in the Z-burst hypothesis are probably both unknown boosting mechanism of the primary neutrinos up to huge energies of hundreds $\mathrm{ZeV}$ and their large flux required at the resonant energy $E_{\nu} \simeq M_{Z}^{2} /\left(2 m_{\nu}\right) \simeq 4.2 \times 10^{21} \mathrm{eV}\left(\mathrm{eV} / m_{\nu}\right)$ well above the GZK cutoff. Such a flux severely challenges conventional source models. Any concomitant photon flux should not violate existing upper limits. The obvious question is then arisen: Where in the Cosmos are these neutrinos coming from? It turns out that currently at energies in excess of $10^{19} \mathrm{eV}$, there are only two good candidate source classes for 
UHE neutrinos: AGNs and GRBs. While hard to detect, neutrinos have the advantage of representing unique fingerprints of hadron interactions and, therefore, UHE neutrinos may initiate the cascades of UHE cosmic rays via very complex chains of Z-burst interactions. Some part of UHE neutrinos may produce, in accretion disk and in a torus of hot gas surrounding the AGN core, the secondary electrons with huge energies, which, in turn, may give rise a secondary flux of the GeV-TeV gammarays. Two basic event topologies can be distinguished: track-like patterns of detected Cherenkov light (hits) which originate from muons produced in charged-current interactions of muon neutrinos (muon channel); spherical hit patterns which originate from the hadronic cascade at the vertex of neutrino interactions or the electromagnetic cascade of electrons from charged current interactions of electron neutrinos (cascade channel). If the charged current interaction happens inside the detector or in case of charged current tau-neutrino interactions, these two topologies overlap which complicates the reconstruction. At the relevant energies, the neutrino is approximately collinear with the muon and, hence, the muon channel is the prime channel for the search for point-like sources of cosmic neutrinos. On the other hand, cascades deposit all of their energy inside the detector and therefore allow for a much better energy reconstruction with a resolution of a few 10\%. Finally, a numerous reports are available at present in literature on expected discovery potential and sensitivity of experiments to neutrino point-like sources. Currently operating high energy neutrino telescopes attempt to detect UHE neutrinos, such as ANTARES which is the most sensitive neutrino telescope in the Northern Hemisphere, IceCube which is worldwide largest and hence most sensitive neutrino telescope in the Southern Hemisphere, BAIKAL, as well as the CR extended experiments of The Telescope Array, Pierre Auger Observatory, and JEM-EUSO mission, etc. Anyhow, with neutrino telescopes of the cubic-kilometer class we are now entering into a sensitivity region where exactly these discoveries will be made is hard to forsee as the predicted fluxes are at the edge of delectability and the uncertainties on the neutrino fluxes are still large. In this regard, it will be of vital interest to compute in the framework of MTBH the high-energy astrophysical neutrino fluxes from aforementioned IMBH-candidates too.

\subsection{UHE neutrino fluxes}

We are now in a position to discuss the predominant cooling mechanism of SPC in terms of neutrino emission. Namely, the neutrino created leave SPC carrying away energy and thus cooling of SPC. Both quark- and pion-condensed proto-matter core cool much more rapidly than (n-p-e)proto-matter core, because of the reason that the simple URCA process can occur in the former both cases, while in (n-p-e)-proto-matter core it is very inefficient for phase space reasons (see below). As before, we denote the quantities referred to SPC by wiggles, while the corresponding quantities of neutron star or quark-star are left without wiggles. The interior temperature or temperature at the surface of proto-matter core is $\widetilde{T}>\frac{100 \mathrm{ZeV}}{k_{B}} \sim 1.2 \times 10^{27} \mathrm{~K}$ (Ter-Kazarian, 2001). The SPC interiors are to a good approximation isothermal, but near the surface the temperature $\widetilde{T}_{e}$ drops rapidly. We may, therefore, adapt a standard discussion of the degenerate-nondegenerate transition region of a neutron star to the corresponding region of SPC. This is quick to estimate $\widetilde{T}_{e}$ and will guide us toward first look at some of the associated physics. So, given the surface temperature of the neutron star $T_{e}: \frac{T_{e}}{T}=1 \times 10^{-2} T_{9}^{-5 / 8}\left(\frac{M}{M_{\odot}}\right)^{1 / 4}\left(\frac{R}{10 \mathrm{~km}}\right)^{-1 / 2}$, where $T_{9}$ denotes the temperature in units of $10^{9} \mathrm{~K}$, and using the approximate fit to Tsuruta's calculation $T_{e} \simeq(10 T)^{2 / 3}$, one may expect that the rough qualitative behavior for the surface temperature $\widetilde{T}_{e}$ of the SPC can be given by $\frac{\widetilde{T}_{e}}{T_{e}} \simeq\left(\frac{\widetilde{T}}{T}\right)^{3 / 8}\left(\frac{\widetilde{M}}{M}\right)^{1 / 4}\left(\frac{\widetilde{R}}{R}\right)^{-1 / 2}$. Therefrom, for the values $\widetilde{R} \simeq \frac{1}{4} R_{g}(?), R \simeq 10 \mathrm{~km}$ and $\widetilde{T} \simeq \widetilde{T}_{100 \mathrm{ZeV}}$, we obtain $\frac{\widetilde{T}_{e}}{T_{e}} \simeq 3.5 \times 10^{7}\left(\frac{\widetilde{M}}{M_{\odot}}\right)^{-1 / 4}$. Setting $T_{e} \simeq 2 \times 10^{6} \mathrm{~K}$, the average surface temperature for the SPCs with the masses $M_{B H} \sim\left(1.1 \times 10^{6}\right.$ to $\left.1.3 \times 10^{10}\right) M_{\odot}$ will then be $\overline{\widetilde{T}}_{e} \simeq 1.2 \times 10^{12} \mathrm{~K}$.

\subsection{URCA reactions}

In contrast to simple URCA reactions $n \rightarrow p+e^{-}+\bar{\nu}_{e}$ and $e^{-}+p \rightarrow n+\nu_{e}$, the nucleon-modified URCA processes can occur only when a bystander neutron must be present to absorb momentum: $n+n \rightarrow n+p+e^{-}+\bar{\nu}_{e}$, and $n+e^{-}+p \rightarrow n+n+\nu_{e}$. Thereby the muon-neutrino emitting reactions may also occur whenever the chemical potential $\mu_{e}>m_{\mu} c^{2}$. But the $\tau$-neutrinos do not occur because of 


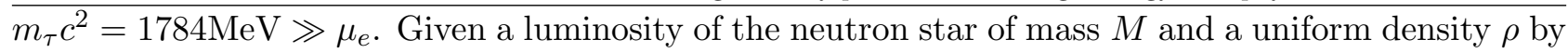
modified URCA reactions with no muons, it is straightforward to estimate a luminosity of SPC (TerKazarian, 2014). Such approach is much less time-consuming than a direct calculation. Note that for each degenerate species of the proto-matter in SPC, only a fraction $\sim k \widetilde{T} / \widetilde{E}_{F}$ can effectively contribute to the cooling rate of SPC because of $d^{3} \widetilde{p}_{i} \rightarrow \widetilde{p}_{F}(i)^{2} d \widetilde{E}_{F}(i) \propto \widetilde{T}$, which for five degenerate species gives $\propto \widetilde{T}^{5}$. Another factor, $\widetilde{T}^{3}$, is due to the antineutrino phase space: $d^{3} \widetilde{p}_{\bar{\nu}} \propto\left(\widetilde{E}_{\bar{\nu}}\right)^{2} d \widetilde{E}_{\bar{\nu}} \propto \widetilde{T}^{3}$. We also should take into account that the temperature that determines the thermal emission from SPC is that at the surface $\widetilde{T}_{e}$ rather than the interior temperature $\widetilde{T}$. The luminosity of SPC, therefore, can approximately be written $\widetilde{L}_{\nu \varepsilon}^{U R C A}=\varepsilon \frac{\widetilde{M}}{M}\left(\frac{\widetilde{\rho}}{\rho}\right)^{-1 / 3}\left(\frac{\widetilde{T}}{T}\right)^{8} L_{\nu}^{U R C A} \simeq \varepsilon \frac{\widetilde{M}}{M}\left(\frac{\widetilde{\rho}}{\rho}\right)^{-1 / 3}\left(\frac{\widetilde{T}_{F}}{T_{F}}\right)^{5}\left(\frac{\widetilde{T}_{e}}{T_{e}}\right)^{3} L_{\nu}^{U R C A}$. The coefficient $\varepsilon$ reads $\varepsilon=\varepsilon_{d} \varepsilon_{\text {trap }}, \quad \varepsilon_{d}=\frac{d}{2 R_{g}} \simeq \theta$, where $d$ is the thickness of the proto-matter disk at the edge of even horizon, $\theta$ is a beaming angle, $\varepsilon_{\text {trap }}$ is the neutrino trapping coefficient. The Fermi energy of each of five fermions, participating in the modified URCA reactions, is $\widetilde{E}_{F i} \sim \widetilde{T}_{F i} \sim \widetilde{\rho}^{1 / 3}$, as well as $E_{F i} \sim T_{F i} \sim \rho^{1 / 3}$. Then $\widetilde{L}_{\nu \varepsilon}^{U R C A}=\varepsilon \frac{\widetilde{M}}{M_{\odot}}\left(\frac{\widetilde{\rho}}{\rho}\right)^{4 / 3}\left(\frac{\widetilde{T}_{e}}{T_{e}}\right)^{3} L_{\nu}^{U R C A}$. The trapping coefficient, $\varepsilon_{\text {trap }}$, arises as the neutrinos are trapped comove with matter and build up a semidegenerate Fermi sea. We might expect that the neutral current reactions will inhibit neutrino transport in SPC and enhance neutrino trapping. Neutrino trapping event forces the liberated energy to be emitted on a much larger diffusion timescale for neutrinos to diffuse out of the proto-matter core. This can be roughly estimated by assuming, in analogy of neutron star, that the coherent scattering in proto-matter core is the dominant opacity source. Namely, the heavy particle of proto-matter core acts nonlinearly as a single particle. Coherent scattering induces a random-walk trajectory for the neutrino in the proto-matter core and proto-matter disk without slightly changing its energy before reaching the surface of SPC. Furthermore, we assume that the mean energy of neutrinos generated via electron capture is comparable to the electron Fermi energy. Thus eventually the average neutrino trapping coefficient in proto-matter medium of SPCs with the masses $M_{B H} \sim\left(1.1 \times 10^{6}\right.$ to $\left.1.3 \times 10^{10}\right) M_{\odot}$ is $\bar{\varepsilon}_{\text {trap }} \simeq 6.36 \times 10^{-20}$. We obtain then (Ter-Kazarian, 2014): $\widetilde{L}_{\nu \varepsilon}^{U R C A}=3.8 \times 10^{50} \varepsilon_{d}\left(\frac{M_{\odot}}{\bar{M}}\right)^{1.75} \mathrm{erg} \mathrm{s}^{-1}$. The average neutrino luminosity of considered SPCs is $\overline{\widetilde{L}}_{\nu \varepsilon}^{U R C A}=5.1 \times 10^{39} \varepsilon_{d} \mathrm{erg} \mathrm{s}^{-1}$.

The flux can be written in terms of luminosity as $J_{\nu \varepsilon}=\widetilde{L}_{\nu \varepsilon} / 4 \pi D_{L}^{2}(z)(1+z)$, where $z$ is the redshift, $D_{L}(z)$ is the luminosity distance depending on the cosmological model. The $(1+z)^{-1}$ is due to the fact that each neutrino with energy $\widetilde{E}_{\nu}^{\prime}$ if observed near the place and time of emission $t^{\prime}$ will be red-shifted to energy $\widetilde{E}_{\nu}=\widetilde{E}_{\nu}^{\prime} R\left(t_{1}\right) / R\left(t_{0}\right)=\widetilde{E}_{\nu}^{\prime}(1+z)^{-1}$ of the neutrino observed at time $t$ after its long journey to us, where $R(t)$ is the cosmic scale factor. Computing the UHE neutrino fluxes in the framework of MTBH, we choose the cosmological model favored today, with a flat universe, filled with matter $\Omega_{M}=\rho_{M} / \rho_{c}$ and vacuum energy densities $\Omega_{V}=\rho_{V} / \rho_{c}$, thereby $\Omega_{V}+\Omega_{M}=1$, where the critical energy density $\rho_{c}=3 H_{0}^{2} /\left(8 \pi G_{N}\right)$ is defined through the Hubble parameter $H_{0}$ : $D_{L}(z)=2.4 \times 10^{28} I(z) \mathrm{cm}$. Here $I(z)=(1+z) \int_{1}^{1+z} d x / \sqrt{2.3+x^{3}}$, and that we have taken the values $H_{0}=70 \mathrm{~km} / \mathrm{s} \mathrm{Mpc}, \Omega_{V}=0.7$ and $\Omega_{M}=0.3$. Combining these equations, the resulting total UHE neutrino flux of cooling of the SPC via simple or modified URCA processes with no muons can be obtained as $J_{\nu \varepsilon}^{U R C A} \simeq 5.22 \times 10^{-8} \frac{\varepsilon_{d}}{I^{2}(z)(1+z)}\left(\frac{M_{\odot}}{M}\right)^{1.75}\left[\mathrm{erg} \mathrm{cm}^{-2} \mathrm{~s}^{-1} \mathrm{sr}^{-1}\right]$, where the neutrino is radiated in a cone with the beaming angle $\theta \sim \varepsilon_{d} \ll 1, I(z)=(1+z) \int_{1}^{1+z} d x / \sqrt{2.3+x^{3}}$. As it is seen, the nucleon modified URCA reactions can contribute efficiently only for extragalactic objects with enough small redshift $z \ll 1$.

\subsection{Pionic reactions}

The pionic reactions allow both energy and momentum to be conserved in the reactions, which are known as an analogue of the simple URCA processes: $\pi^{-}+n \rightarrow n+e^{-}+\bar{\nu}_{e}, \pi^{-}+n \rightarrow n+\mu^{-}+\bar{\nu}_{\mu}$, and two inverse processes. As in the modified URCA reactions, the total rate for all four processes is essentially four times the rate of each reaction alone. Muons are already present when pions appear. Note that there are two fewer fermions participating in the reactions than in the modified URCA reactions. So the phase-space factor varies as $T^{6}$ rather than $T^{8}$. We may obtain then $\widetilde{L}_{\nu \varepsilon}^{\pi}=\varepsilon \frac{\widetilde{M}}{M} \frac{\rho}{\widetilde{\rho}}\left(\frac{\widetilde{T}_{F}}{T_{F}}\right)^{3}\left(\frac{\widetilde{T}_{e}}{T_{e}}\right)^{3} L_{\nu}^{\pi}$, where $\widetilde{T}_{F}=\widetilde{E}_{F} / k_{B}, \quad L_{\nu}^{\pi}$ refers to the neutron star: $L_{\nu}^{\pi} \sim 1.5 \times 10^{46} \vartheta^{2} \frac{M}{M_{\odot}} \frac{\rho_{n u c}}{\rho} T_{9}^{6} \operatorname{erg~s}^{-1}$, and $\vartheta \sim 0.3$ is an angle measuring the degree of pion condensation. Hence $\widetilde{L}_{\nu \varepsilon}^{\pi}=5.78 \times 10^{58} \varepsilon_{d}\left(\frac{M_{\odot}}{\widetilde{M}}\right)^{1.75} \operatorname{erg~s}^{-1}$, 
and that the average neutrino luminosity of considered SPCs is $\widetilde{L}_{\nu \varepsilon}^{\pi}=7.7 \times 10^{47} \varepsilon_{d} \mathrm{erg} \mathrm{s}^{-1}$. Therefrom the total UHE neutrino flux reads $J_{\nu \varepsilon}^{\pi} \simeq 7.91 \frac{\varepsilon_{d}}{I^{2}(z)(1+z)}\left(\frac{M_{\odot}}{\widetilde{M}}\right)^{1.75} \mathrm{erg} \mathrm{cm}^{-2} \mathrm{~s}^{-1} \mathrm{sr}^{-1}$. So, the average flux is $\bar{J}_{\nu \varepsilon}^{\pi} \simeq 1.1 \times 10^{-10} \varepsilon_{d} / I^{2}(z)(1+z) \mathrm{erg} \mathrm{cm}^{-2} \mathrm{~s}^{-1} \mathrm{sr}^{-1}$. As it is seen, the resulting total energy-loss rate will then be dramatically larger due to the pionic reactions rather than the modified URCA processes.

\subsection{Quark reactions}

In the superdense proto-matter medium the distorted quark Fermi energies are far below the charmed c-, t- , and b- quark production thresholds. Therefore, only up, down, and strange quarks are present. The $\beta$ equilibrium is maintained by reactions like $d \rightarrow u+e^{-}+\bar{\nu}_{e}, \quad u+e^{-} \rightarrow d+\nu_{e}$, and $s \rightarrow u+e^{-}+\bar{\nu}_{e}, \quad u+e^{-} \rightarrow s+\nu_{e}$, which are $\beta$ decay and its inverse. These reactions constitute simple URCA processes, in which there is a net loss of a $\nu_{l} \bar{\nu}_{l}$ pair at nonzero temperatures. In this application a sufficient accuracy is obtained by assuming $\beta$-equilibrium and that the neutrinos not to be retained in the medium of $\Lambda$-like proto-matter. The above quark reactions proceed at equal rates in $\beta$ equilibrium, where the participating quarks must reside close to their Fermi surface. Hence, the total energy of flux due to simple URCA processes is rather twice than of each alone. Our purpose here is to adapt in sufficient approximation a discussion of ordinary quark matter in neutron star to the corresponding region of quark proto-matter in SPC, where a rearrangement of vacuum state causes a shift of zero point energy. Accordingly, the energy-loss rate of the SPC due to neutrino emission process $d \rightarrow u+e^{-}+\bar{\nu}_{e}$ can be generalized in the form $\widetilde{\epsilon}_{\bar{\nu} \varepsilon}^{q}=6 V^{-1} \varepsilon\left(\prod_{i=1}^{4} V \int \frac{d^{3} \widetilde{p}_{i}}{(2 \pi)^{3}}\right) \widetilde{E}_{\bar{\nu}} V(2 \pi)^{4} \delta^{(4)}\left(\widetilde{p}_{d}-\right.$ $\left.\widetilde{p}_{\bar{\nu}_{e}}-\widetilde{p}_{u}-\widetilde{p}_{e}\right) \frac{|M|^{2}}{\prod_{i=1}^{4} 2 \widetilde{E}_{i} V} S$. Here $\widetilde{p}_{i}$ and $\widetilde{E}_{i}$ denote the distorted momentum and energy of a particle of the given species of proto-matter. The four-vectors $\widetilde{p}_{i}$ are numbered as $i=1,2,3,4 \equiv d, \bar{\nu}_{e}, u, e^{-}, \quad V$ is the normalization volume, $|M|^{2}$ is the squared invariant amplitude averaged over the initial $d$-quark spin and summed over the final spins of the $u$ quark and the electron. Also, $S=f_{d}\left(1-f_{u}\right)\left(1-f_{e}\right)$ is the statistical factor, $f_{i} \equiv\left[\exp \widetilde{\beta}\left(\widetilde{E}_{i}-\widetilde{\mu}_{i}\right)+1\right]^{-1}$ is the fraction of phase space occupied at energy $\widetilde{E}_{i}$ and distorted chemical potential $\widetilde{\mu}_{i}=\left(1+8 \alpha_{c} / 3 \pi\right) c \widetilde{p}_{F i}, \widetilde{p}_{F i}$ is the Fermi momentum. This is given to lowest order in the QCD interaction coupling constant $\alpha_{c}$. The blocking factors $1-f_{i}$ accounting for the distribution of final states reduce the reaction rate, which ensure that the exclusion principle is obeyed; the factor 3 takes account of three color degrees of freedom, and 2 is the spin of the initial $d$ quark. Since typical Fermi energies of quarks are higher than the electron rest mass, a slight difference between $\widetilde{\mu}_{d}$ and $\widetilde{\mu}_{e}$ (or $\widetilde{\mu}_{s}$ and $\widetilde{\mu}_{u}$ ) implies that the electrons will generally have a relativistic Fermi energy. The interaction Lagrangian density is given in the standard current-current form $\mathcal{L}_{I}^{W}=\frac{G}{\sqrt{2}} \cos \theta_{C} \bar{u} \gamma^{\mu}\left(1-\gamma_{5}\right) d \bar{e} \gamma_{\mu}\left(1-\gamma_{5}\right) \nu_{e}$, where the weak-coupling constant is $G \simeq 1.435 \times$ $10^{-40} \mathrm{erg} \mathrm{cm}^{3}, \quad \theta_{C}$ is the Cabibbo angle $\left(\cos ^{2} \theta_{C} \simeq 0.948\right)$. The squared invariant averaged amplitude can be calculated in case of proto-matter medium to be $\frac{1}{2} \sum_{\sigma_{1}, \sigma_{3}, \sigma_{4}}\left|M_{f i}\right|^{2}=64 G^{2} \cos ^{2} \theta_{C}\left(\widetilde{p}_{1}\right.$. $\left.\widetilde{p}_{2}\right)\left(\widetilde{p}_{3} \cdot \widetilde{p}_{4}\right)$. As the neutrinos are produced thermally, we can neglect the neutrino momentum in the momentum conservation law, and that to the lowest order in $\alpha_{c}$ we can use an approximate formula $|M|^{2}=\frac{1024 \alpha_{c}}{3 \pi} G^{2} \cos ^{2} \theta_{C} \widetilde{E}_{d} \widetilde{E}_{\bar{\nu}} \widetilde{E}_{u} \widetilde{E}_{e}\left(1-\frac{\left|\widetilde{\mathbf{p}}_{d}\right|}{\widetilde{E}_{d}} \cos \theta_{d \bar{\nu}}\right)$, where $\cos \theta_{d \bar{\nu}} \equiv \hat{\widetilde{p}}_{d} \cdot \hat{\widetilde{p}}_{\bar{\nu}}, \quad \hat{p}_{i} \equiv \frac{\widetilde{\mathbf{p}}_{i}}{\left|\widetilde{p}_{i}\right|}$. We may further set $\widetilde{E}_{i} \simeq \widetilde{p}_{i}$ because all the particles are relativistic, and that $\widetilde{p}_{d} \sim \widetilde{p}_{u} \quad\left(\widetilde{p}_{e}<<\widetilde{p}_{d}\right)$. Also all the $\widetilde{p}_{i}$ (except $\widetilde{p}_{\bar{\nu}}$ ) can be set equal $\widetilde{p}_{F}(i)$ and removed from the integral. To estimate the magnitude of the emissivity, we follow the original argument of (Ter-Kazarian, 2014), which can be applied to both neutron star and SPC, and that assume for distorted Fermi momenta $\widetilde{p}_{F}(d) \simeq \widetilde{p}_{F}(u) \simeq \widetilde{p}_{F}(s)$ as in non-interacting case, such that $\widetilde{p}_{F}(e) \simeq 3^{(1 / 3)} \widetilde{Y}_{e}^{(1 / 3)} \widetilde{p}_{F}(q)$, where $\widetilde{Y}_{e} \simeq \frac{\widetilde{n}_{e}}{\widetilde{n}_{b}}, \quad \widetilde{n}_{i}$ is the distorted concentration of particles. We may also set the value $\alpha_{c} \simeq 0.1$ as rather being a reasonable order of magnitude estimate, and $\widetilde{Y}_{e} \simeq 0.01$ as typical value of what is expected for superdense proto-matter. A standard evaluation of integrals in the complex plane, as well as taking into account that for each degenerate species only a fraction $\widetilde{T} / \widetilde{T}_{F}$ effectively contribute to the cooling rate, and that there are one such initial species and two such final species, finally we arrive at the spectral flux of the UHE antineutrinos $\frac{d J_{\nu \varepsilon}^{q}}{d y_{2}} \simeq 0.41 \frac{\varepsilon_{d}}{I^{2}(z)(1+z)}\left(\frac{M_{\odot}}{\widetilde{M}}\right)^{1.75} \frac{y_{2}^{3}\left(\pi^{2}+y_{2}^{2}\right)}{e^{y}+1} \mathrm{erg} \mathrm{cm}^{-2} \mathrm{~s}^{-1} \mathrm{sr}^{-1}$.

Similar reasonings can be applied for the process $u+e^{-} \rightarrow d+\nu_{e}$, where $\widetilde{E}_{d}+\widetilde{E}_{\nu}=\widetilde{E}_{e}+\widetilde{E}_{u}$, $\widetilde{E}_{d}-\widetilde{E}_{u} \simeq \widetilde{E}_{e} \cos \theta_{e u}-\widetilde{E}_{\nu} \cos \theta_{d \nu}$. Taking into account that $d \Omega_{e} \simeq\left(1 / \widetilde{E}_{e}\right) d \widetilde{E}_{d} d \varphi_{e}$, so 

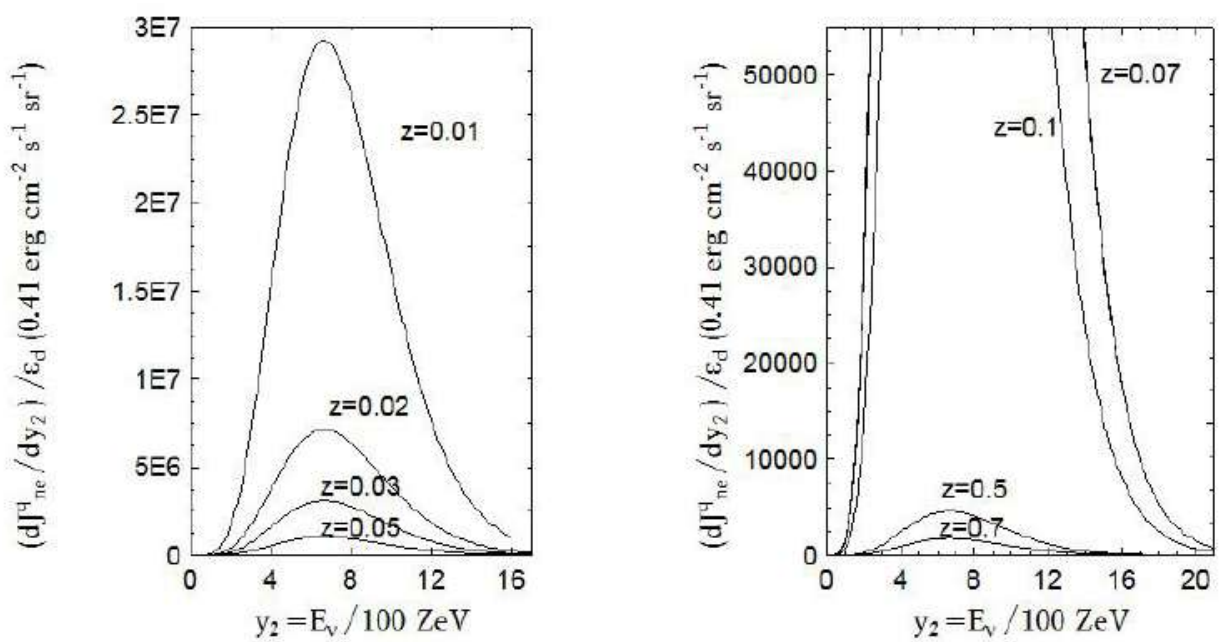

Figure 5. The spectral fluxes of UHE antineutrinos for different redshifts from quark reactions.

$\int \frac{\widetilde{p}_{\nu} \widetilde{p}_{u} \widetilde{p}_{e}}{\widetilde{E}_{d}} d \Omega_{\nu} d \Omega_{u} d \Omega_{e} d \widetilde{E}_{\nu} d \widetilde{E}_{u} d \widetilde{E}_{e}=\int \widetilde{E}_{\nu} d \varphi_{e} d \Omega_{\nu} d \Omega_{u} d \widetilde{E}_{d} d \widetilde{E}_{\nu} d \widetilde{E}_{u} d \widetilde{E}_{e}$, we obtain the resulting spectral UHE neutrino flux: $\frac{d J_{\nu \varepsilon}^{q}}{d y_{1}} \simeq 0.1 \frac{\varepsilon_{d}}{I^{2}(z)(1+z)}\left(\frac{M_{\odot}}{\widetilde{M}}\right)^{1.75} \frac{y_{1}^{4}\left(\pi^{2}+y_{1}^{2}\right)}{e^{y_{1}+1}} \mathrm{erg} \mathrm{cm}^{-2} \mathrm{~s}^{-1} \mathrm{sr}^{-1}$, where $y_{1} \equiv \widetilde{E}_{\nu} \widetilde{\beta}$. A different $\widetilde{E}_{\nu}$ dependence is due to the partial restriction of the electron's phase space which introduces an extra factor of $\left(\widetilde{E}_{e} \widetilde{\beta}\right)^{-1}$. But, as far as the neutrino flux is independent of the electron's Fermi energy, a potential source of ambiguity is eliminated. Consequently, both fluxes above yield the total UHE neutrino flux $J_{\nu \varepsilon}^{q} \simeq 70.68 \frac{\varepsilon_{d}}{I^{2}(z)(1+z)}\left(\frac{M_{\odot}}{\widetilde{M}}\right)^{1.75} \mathrm{erg} \mathrm{cm}^{-2} \mathrm{~s}^{-1} \mathrm{sr}^{-1}$, and that eventually the average flux is $\bar{J}_{\nu \varepsilon}^{q} \simeq 9.46 \times 10^{-10} \varepsilon_{d} / I^{2}(z)(1+z) \mathrm{erg} \mathrm{cm}^{-2} \mathrm{~s}^{-1} \mathrm{sr}^{-1}$.

\subsection{Simulation}

For simulation we use the data of AGN/BH mass and luminosity estimates for 377 black holes which are mostly based on the virial assumption for the broad emission lines, with the broad-line region size determined from either reverberation mapping or optical luminosity. Additional black hole mass estimates based on properties of the host galaxy bulges, using either the observed stellar velocity dispersion or using the fundamental plane relation. Since the aim is to have more than a thousand of realizations, each individual run is simplified, with a use of previous algorithm of the SPC-configurations as a working model. Computing the corresponding PRTs, seed black hole intermediate masses and total neutrino fluxes, a main idea comes to solving an inverse problem. Namely, by the numerous reiterating integrations of the state equations of SPC-configurations we determine those required central values of particle concentration $\widetilde{n}(0)$ and ID-field $x(0)$, for which the integrated total mass of configuration has to be equal to the black hole mass $M_{B H}$ given from observations. Along with all integral characteristics, the radius $R_{d}$ also computed which is in use for calculating $M_{B H}^{\text {Seed }}, T_{B H}, z^{\text {Seed }}$, and $J_{\nu \varepsilon}^{i}$, respectively. The Fig. 4 gives the intermediate seed masses $M_{B H}^{\text {Seed }} / M_{\odot}$ versus the present masses $M_{B H} / M_{\odot}$ of 337 black holes, on logarithmic scales. For the present masses $M_{B H} / M_{\odot} \simeq 1.1 \times 10^{6}$ to $1.3 \times 10^{10}$, the computed intermediate seed masses are ranging from: $M_{B H}^{\text {Seed }} / M_{\odot} \simeq 26.4$ to $2.9 \times 10^{5}$.

The spectral fluxes of the UHE antineutrinos and neutrinos for different redshifts from quark reactions are plotted respectively on the Fig. 5 and Fig. 6. The computed neutrino fluxes are ranging from: 1) (quark reactions) $-J_{\nu \varepsilon}^{q} / \varepsilon_{d}\left[\mathrm{erg} \mathrm{cm} \mathrm{cm}^{-2} \mathrm{~s}^{-1} \mathrm{sr}^{-1}\right] \simeq 8.29 \times 10^{-16}$ to $3.18 \times 10^{-4}$, with the average $\bar{J}_{\nu \varepsilon}^{q} \simeq 5.53 \times 10^{-10} \varepsilon_{d}\left[\mathrm{erg} \mathrm{cm}^{-2} \mathrm{~s}^{-1} \mathrm{sr}^{-1}\right] ; 2$ ) (pionic reactions) $-J_{\nu \varepsilon}^{\pi} \simeq 0.112 J_{\nu \varepsilon}^{q}$, with the average $J_{\nu \varepsilon}^{\pi} \simeq 3.66 \times 10^{-11} \varepsilon_{d}\left[\mathrm{erg} \mathrm{cm}^{-2} \mathrm{~s}^{-1} \mathrm{sr}^{-1}\right]$; and 3) (modified URCA processes) $-J_{\nu \varepsilon}^{U R C A} \simeq$ $7.39 \times 10^{-11} J_{\nu \varepsilon}^{q}$, with the average $\bar{J}_{\nu \varepsilon}^{U R C A} \simeq 2.41 \times 10^{-20} \varepsilon_{d}\left[\mathrm{erg} \mathrm{cm}^{-2} \mathrm{~s}^{-1} \mathrm{sr}^{-1}\right]$. To render our discussion 

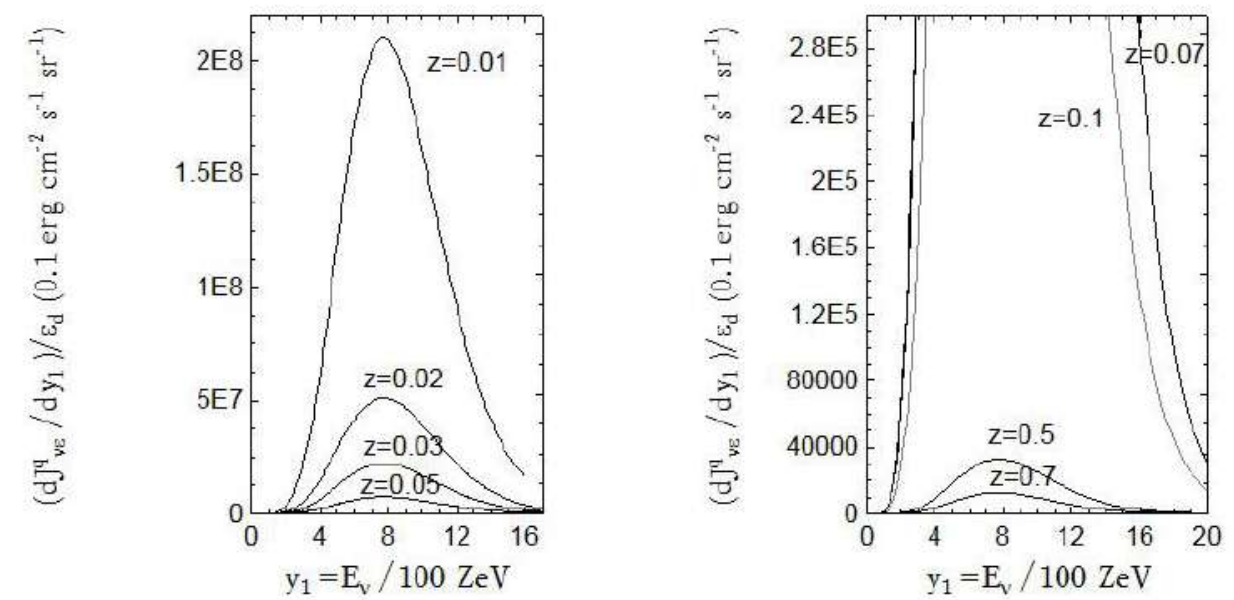

Figure 6. The spectral fluxes of UHE neutrinos for different redshifts from quark reactions.

here a bit more transparent and to obtain some feeling for the parameter $\varepsilon_{d}$ we may estimate $\varepsilon_{d} \simeq$ $1.69 \times 10^{-10}$, just for example only, for the suppermassive black hole of typical mass $\sim 10^{9} M_{\odot}\left(2 R_{g}=\right.$ $5.9 \times 10^{14} \mathrm{~cm}$ ), and so $d \sim 1 \mathrm{~km}$. But the key problem of fixing the parameter $\varepsilon_{d}$ more accurately from experiment would be an important topic for another investigation elsewhere. Reflecting upon the results far obtained, we draw a statement that the AGNs are favored as promising pure neutrino sources because the computed neutrino fluxes are highly beamed along the plane of accretion disk, and peaked at high energies and collimated in smaller opening angle. The neutrinos are able to stream freely out of SPC, and the bulk of liberated binding energy of proto-matter must be converted into other forms of internal energy rather than being released immediately in the form of escaping neutrinos. Such neutrinos may initiate the cascades of UHE cosmic rays via very complex chains of Z-burst interactions. Some part of UHE neutrinos may produce, in accretion disk and in a torus of hot gas surrounding the AGN core, the secondary electrons with huge energies, which, in turn, may give rise a secondary flux of the GeV-TeV gamma-rays. Above said was sharpened by the recent surprising announcement of the first high-energy neutrino event by the IceCube Neutrino Observatory (Collaboration, 2018). With the very large volume neutrino telescope optimized for the $\mathrm{TeV}$ energy range, they have traced a neutrino with the energy approximately $300 \mathrm{TeV}$ that hit their Antarctica-based detector in September 2017 back to its point of origin in a blazar, TXS 0506+056, the 3.7 billion light-years away. This constitutes the first use of a neutrino detector to locate an object in space.

\section{On the physical nature of the source of ultraluminous X-ray pul- sations}

The physical nature of the accreting off-nuclear point sources in nearby galaxies, so-called the ultraluminous X-ray sources (ULXs), has been an enigma because of their high energy output characterized by super-Eddington luminosities up to two orders of magnitude higher than those observed from Galactic X-ray binaries, and unusual soft X-ray spectra with blackbody emission around $\lesssim 0.3$ $\mathrm{keV}$ and a downturn above $\sim 5 \mathrm{keV}$. In spite of significant efforts in more than three decade since the discovery of ULXs, the astronomers have not yet clarified their nature. Without care of the historical justice and authenticity, it should be emphasized that the ULX sources have attracted a great deal of observational and theoretical attention, in part because their luminosities suggest that they may harbor intermediate mass black holes (IMBHs) with an ubiquitous feature of the mass fits of more than $10^{2}-10^{4} M_{\odot}$. A strong argument in favor of IMBHs is the presence of a soft, $0.1-0.2 \mathrm{keV}$ 
component in their spectra. The hyperluminous X-ray sources with luminosities $\geq 10^{41} \mathrm{erg} \mathrm{s}^{-1}$ are thought to be amongst the strongest IMBH candidates. Assuming the emission is isotropic, in general, the extreme luminosities of ULXs suggest either the presence of IMBHs and sub-Eddington accretion, or stellar-mass black holes $\left(M \leq 10 M_{\odot}\right)$ that are either breaking or circumventing their Eddington limit via somewhat geometric beaming of accretion flow. The latter remains still a relatively poorly understood regime. The brightest ULXs (with $L_{X}>5 \times 10^{40} \mathrm{erg} \mathrm{s}^{-1}$ ), located within $100 \mathrm{Mpc}$, are identified in a cross correlation of the 2XMM-DR1 and RC3 catalogues. These objects seemed to be amongst the most plausible candidates to host larger, IMBHs. But, future improved observations are needed yet to decide on the issue. The quasi-periodic oscillations (QPOs) and frequency breaks in XMM-Newton power-density spectra of ULXs with luminosity, $L_{X} \geq 10^{40} \mathrm{erg} \mathrm{s}^{-1}$, suggest that the black hole masses are more consistent with IMBHs than stellar-mass black holes. Or, using high sensitive dataset in the Fe K region obtained in scope of a long Suzaku program on Holmberg IX X-1 for any luminous, isolated ULX to date, it is found no statistically significant narrow atomic features in either emission or absorption across the 5-9 keV energy range. Therefore, the models of spherical super-Eddington accretion can be rejected, as can wind-dominated spectral models. The lack of iron emission implies that the stellar companion in sub-Eddington accretion onto an IMBH is unlikely to be launching a strong wind, and therefore the black hole must primarily accrete via roche-lobe overflow. There are also several other new results of more recent studies: for example, if the transferred mass is efficiently converted to X-ray luminosity (with disregard of the classical Eddington limit) or if the X-rays are focused into a narrow beam then binaries can form extreme ULXs with the X-ray luminosity of $L_{X} \gtrsim 10^{42} \mathrm{erg} \mathrm{s}^{-1}$. It was envisaged that these systems are not only limited to binaries with stellar-origin black hole accretors, but also can be identified with neutron star systems. For the latter the typical donors are evolved low-mass $\left(2 M_{\odot}\right)$ helium stars with Roche lobe overflow rate of $\sim 10^{-2} M_{\odot \mathrm{yr}^{-1}}$. This study does not prove that any particular extreme ULX is a regular binary system. Combining binary population synthesis and detailed mass-transfer models, one concludes that the binary system that formed M82X-2 is most likely less than $50 \mathrm{Myr}$ old and contains a donor star which had an initial mass of approximately $8-10 M_{\odot}$, while the NS's progenitor star had an initial mass in the $8-25 M_{\odot}$ range. The resulting luminosity of NS pulsars may can reach values of the order of $10^{40} \mathrm{erg} \mathrm{s}^{-1}$ for the magnetar-like magnetic field, since the equilibrium, where the Alfvén radius matches the co-rotation radius, indicates a magnetic field of $B \gtrsim 10^{14} \mathrm{G}$, and long spin periods $(P \gtrsim 1.5 s)$. They conclude that a substantial part of ULXs are accreting neutron stars in binary systems.

All these proposals have own advantages and difficulties. Nevertheless, no single theory has been invented yet which successfully addresses the solution to the problems involved. The aforementioned studies are not exception to the rule that as phenomenological approaches they suffer from own difficulties. Namely, they are strongly model dependent and subject to many uncertainties. The physics is obscured by multiple assumptions and proliferation of a priori free parameters involved, while a consistent complete theory would not have so many free parameters. So, the issue is still controversial, and these exotic results and conclusions are still lame and for sure not proven by the authors. For example, one evokes the possibility that the transferred mass is efficiently accreted onto a compact object and converted to X-ray luminosity in the full range of possible mass accretion rates, which is in contrast with the generally accepted view that the conversion efficiency decreases with increasing mass accretion rate. Or, in case of model of the accretion column, a necessary condition for the X-ray luminosity to exceed the Eddington limit is a certain degree of asymmetry in the distribution of matter over the Alfven surface, which is a qualitative picture, perhaps, but not quantitative one, or a $B \gtrsim 10^{14} \mathrm{G}$ magnetic field of a magnetar-like pulsar is rather above the quantum limit, etc. We will not be concerned here with the actual discussion of these results, which require further extensive and careful investigations, in order could verify the proposed estimates and constrain model parameters, and consequently could lead to a consistent theory. There were still many open key questions arisen inevitably that we have no understanding of ULX physics. A main physical issue whether ULXs are powered by IMBHs or normal stellar black holes to date is unresolved, primarily because we do not have dynamical mass measurements of the compact objects that power ULXs. So, it is premature to draw conclusions and only time will tell whether any of these intriguing proposals is correct and which 
of the hypothesized ULX scenario is actually realized in nature.

\subsection{The ultraluminous X-ray pulsations}

The most striking is the recent revolutionary NuSTAR discovery of the first rare and mighty ultraluminous X-ray pulsations with the maximum luminosity $\widetilde{L}(3-30 \mathrm{keV})=4.9 \times 10^{39} \mathrm{erg} \mathrm{s}^{-1}$, of average period $1.37 \mathrm{~s}$ with a 2.5-day sinusoidal modulation, coming from an ultraluminous source, NuSTAR J095551+6940.8, located nearby starburst galaxy M82 (NGC 3034) (Ter-Kazarian, 2016b). The pulsed emission centroid is spatially consistent with the location of a variable M82X-2 which further secures the association of the pulsating source with M82X-2. The NuSTAR finding will significantly enhance our understanding of the ULX Universe which currently we do not have. Detection of coherent pulsations, a binary orbit, and spin-up behaviour indicative of an accretion torque unambiguously, allow to feature the M82X-2 as mass-exchange binary that contains a nondegenerate secondary donor star. Eventually, in addition to the orbital modulation, about an evident linear spin-up of the pulsar was reported, with $\dot{p} \simeq-2 \times 10^{-10} \mathrm{~s} / \mathrm{s}$ over the interval from modified Julian days 56696 to 56701 when the pulse detection is most significant. Phase connecting the observations enables detection of a changing secular spin-up rate over a longer timespan as well as erratic variations. Future observations will show whether the current spin-up rate is secular. The spin-up to luminosity ratio $10^{-50}(\mathrm{erg} \cdot \mathrm{s})^{-1}$ is an order of magnitude lower than the typical ratio observed in the X-ray pulsars, which makes an interpretation of the data in terms of a strongly magnetized X-ray pulsar quite challenging. In absolute terms, this spin-up rate is orders of magnitude higher than the values measured in the usual accretion powered X-ray pulsars.

\subsection{Key objectives}

A current understanding of NuSTAR discovery is quite complicated. At first glance it seems as though the NuSTAR team has demonstrated that the super-Eddington accretion is also possible in ULXs hosting a neutron star, because it is generally believed that the pulsating X-ray sources are magnetic neutron stars which are accreting matter from a binary companion. Therefore, it seems that there is nothing left but M82X-2, which until recently astronomers had thought was a black hole, is the brightest magnetic neutron star system ever recorded. This point of view is widely quoted in literature and, at first sight, seems eminently reasonable. However, deeper examination raises several disturbing questions, if the above result is really valid. Even though, if for a moment we take the classical accreting neutron star pulsar system as a basic assumption, note that this model is not flawless. The actual mechanism by which pulsars convert the rotational energy of the neutron star into the observed pulses is poorly understood. Many theoretical models have been proposed that account for such features, but no single one is compelling.

Explaining periodic source M82X-2 that obviously has black hole energetics with a $\sim 1.4 M_{\odot}$ compact object using the accreting neutron star pulsar systems is extremely challenging, because of several problems we encountered. The difficulty becomes apparent when one follows the three objections, which together constitute a whole against the claim that M82X-2, perhaps, is a common pulsar: 1) The NuSTAR team discovery is the most extreme violation of the so-called Eddington limit, i.e. the pulsed luminosity of M82X-2 reaches about $\sim 26.9$ times brighter than the theoretical threshold at the spherical accretion for $\sim 1.4 M_{\odot}$ stellar-mass black holes where the outward pressure from radiation balances the inward pull of gravity of the pulsar. The accretion is inhibited once radiation force is equal or grater than gravity force. 2) The difficulty is brought into sharper focus by considering the association with M82X-2, which is featured with high luminosity $\left(\simeq 1.8 \times 10^{40} \mathrm{erg} \mathrm{s}^{-1}\right)$ of additional persistent continuous broad X-ray radiation observed earlier from its active state. This more compelling argument in somehow or other implies the luminosity $\sim 100$ times if compared to the Eddington limit. Such a collimation $(\sim 100)$, which is comparable to that obtained for black holes (e.g., ??), would be needed to explain M82X-2 as beamed radiation from neutron star. 3) Equally noteworthy that the centroid of the persistent X-ray emission is between M82X-2 and M82X-1. If M82X-1 is indeed harbors plausible IMBH, we expect the similarity of the persistent X-ray properties of the M82X-1 and M82X-2 to imply that the non-pulsed emission from the latter originates in the 
accretion disc, as it must in the black hole M82X-1. In this sense, the NuSTAR discovery is unexpected and still hard to be explained in the context of magnetic neutron star pulsar model. The fraction of ULXs powered by neutron stars must be considered highly uncertain and many details of this scenario remain poorly understood. Added to this was the fact that NuSTAR finding may indeed not be rare in the ULX population. In the future astronomers also will look at more ULXs, and it is a matter of time until they could prove an expected ubiquitous feature of even more energetic ULX pulsations, as being common phenomena in the Universe. If confirmed, this would support a scenario in which the more ULXs beat with the pulse of black holes rather than magnetized neutron star systems. What if NuSTAR detection might radically change one's view of Nature. With this perspective in sight, it is wise to place constraints on the likelihood of the magnetic neutron star pulsar systems. So, it is becoming increasingly important that in the case of M82X-2 the model of common pulsar will be tested critically.

\subsection{Result}

Putting apart the discussion of inherent problems of the mass scaling of the black holes in ULXs, which is beyond the scope of this report, we approach the M82X-2 issue from the standpoint of black holes rather than magnetic neutron star pulsar systems (Ter-Kazarian, 2016b). To reconcile the observed unusual high pulsed luminosity with the above mentioned violation of the Eddington limit, we examine the physics which is at work in ultraluminous pulsations by assuming that M82X-2 is being a spinning intermediate-mass black hole (SIMBH). If a black hole of intermediate mass will be an exact law of Nature, it is certainly an attractive scenario. Fore example, it was suggested that the M82X-2 is a binary system with a black hole accretor. Assuming the persistent emission is isotropic, the $\mathrm{X}$-ray luminosity $\simeq 10^{40} \mathrm{erg} \mathrm{s}^{-1}$ implies that the compact object is a $>100 M_{\odot} \mathrm{IMBH}$ in the low/hard state. However, one may be tempted to argue truly that most of the issues and objections raised above cannot be solved in the framework of PBHMs. The coherent periodicity obviously rules out PBHM, because 1) black holes do not radiate; 2) the spinning black holes are axisymmetric and have no internal structure on which to attach a periodic emitter. Orbital motion, whether modulating some emission mechanism directly or exciting short-period pulsations, would decay very quickly due to gravitational radiation. With this in mind, we revisited the MTBH which completes PBHM by exploring the most important processes of spontaneous breaking of gravitation gauge symmetry and rearrangement of vacuum state at huge energies. One of the purposes of the report (Ter-Kazarian, 2016b) is to motivate and justify the further implications of MTBH framework to circumvent the alluded obstacles without the need for significant breaking of Eddington limit.

\subsection{Axisymmetric space $V_{4}$ in $3+1$ formalism}

As in the case of neutron stars, we expect that accreting black holes are fast spinning objects. For the self-contained arguments, we need to extend the preceding algorithm of non-spinning MBHM to its spinning counterpart, which is almost a matter of routine, to change the geometry of static SPC to a more general one, describing axisymmetric rotating SPC. Here we will collect together the results which are required later. The non-spinning SPC is static and spherically symmetric. So, one needs to be clear about more general geometry which can describe rotating axisymmetric SPCs. The principle foundation of the spinning configurations first comprises the following additional distinctive features with respect to non-spinning ones: 1) Rapid rotation causes the shape of the SPC to be flattened by centrifugal forces - flattened at poles and buldged at equator (oblate spheroid, which is second order effect in the rotation rate). 2) A rotating massive SPC drags space and time around with it. The local inertial frames are dragged by the rotation of the gravitational field, i.e. a gyroscope orbiting near the SPC will be dragged along with the rapidly rotating SPC. This is probably the most remarkable feature that could serve as a link with the general description of spacetime. Beside the geodetic procession, a spin of the body produces in addition the Lense-Thirring procession. To look into the future, measurement of the gyrogravitational ratio of particle would be a further step towards probing the microscopic origin of gravity. Let the world coordinate $t\left(=x^{0}\right)$ be the time (in units of $\mathrm{c}$ ), and $\phi\left(=x^{1}\right)$ be the azimuthal angle about the axis of symmetry. Moreover, 
a metric of a two-space, $\left(x^{2}, x^{3}\right)$, can always be diagonalized. Since the source of gravitational field has motions that are pure rotational about the axis of symmetry, then the energy-momentum tensor as the source of the metric will have the same symmetry. Namely, the space $\mathcal{M}_{4}$ would be invariant against simultaneous inversion of time $t$ and azimuthal angle $\phi$. The $3+1$ formalism is the most commonly used approach in which, as usual, spacetime is decomposed into the one parameter family of space-like slices - the hypersurfaces $\Sigma_{t}$. The study of a dragging effect is assisted by incorporating with the soldering tools in order to relate local Lorentz symmetry to curved spacetime. These are the linear frames and forms in tangent fiber-bundles to the external general smooth differential manifold, whose components are so-called tetrad (vierbein) fields. Whereas, the $\mathcal{M}_{4}$ has at each point a tangent space, $T_{x} \mathcal{M}_{4}$, spanned by the anholonomic orthonormal frame field, $e$, as a shorthand for the collection of the 4-tuplet $\left(e_{0}=\exp (-\nu)\left(\partial_{t}+\omega \partial_{\phi}\right), e_{1}=\exp (-\psi) \partial_{\phi}, e_{2}=\exp \left(-\mu_{2}\right) \partial_{2}, e_{3}=\exp \left(-\mu_{3}\right) \partial_{3}\right)$, where $e_{a}=e_{a}{ }^{\mu} \partial_{\mu}, \quad e_{a}^{\mu}$ is the the soldering form between the tangent space and the spacetime manifold. This is called a Bardeen observer, locally nonrotating observer, or the local Zero AngularMomentum Observers (ZAMO), i.e. observers whose worldlines are normal to the hypersurfaces defined by constant coordinate time, $\widetilde{t}=$ const, also called Eulerian observers. Here we use Greek alphabet $(\mu, \nu, \rho, \ldots=0,1,2,3)$ to denote the holonomic world indices related to $V_{4}$, and the first half of Latin alphabet $(a, b, c, \ldots=0,1,2,3)$ to denote the anholonomic indices related to the tangent space. The frame field, $e$, then defines a dual vector, $\vartheta$, of differential forms, $\vartheta=\left(\begin{array}{c}\vartheta^{0}=\exp \nu d \widetilde{t} \\ \vartheta^{1}=\exp \psi(d \psi-\omega d \widetilde{t}) \\ \vartheta^{2}=\exp \mu_{2} d \widetilde{x}^{2} \\ \vartheta^{3}=\exp \mu_{3} d \widetilde{x}^{3}\end{array}\right)$, as a shorthand for the collection of the $\vartheta^{b}=e^{b}{ }_{\mu} d \widetilde{x}^{\mu}$, whose values at every point form the dual basis, such that $\left.e_{a}\right\rfloor \vartheta^{b}=\delta_{a}^{b}$, where $\rfloor$ denoting the interior product, namely, this is a $C^{\infty}$-bilinear map \rfloor$: \Omega^{1} \rightarrow \Omega^{0}$ with $\Omega^{p}$ denotes the $C^{\infty}$-modulo of differential p-forms on $V_{4}$. In components $e_{a}{ }^{\mu} e^{b}{ }_{\mu}=\delta_{a}^{b}$. One can also consider general transformations of a local Lorentz group, taking any frame field, $e$, into any other set, $e^{\prime}$, of four linearly independent fields: $e_{a}^{\prime}=\Lambda_{a}^{b} e_{b}$. Here and throughout the notation, $\left\{e_{a}, \vartheta^{b}\right\}$, will be used for general linear frames. The norm $d \widetilde{s}$ then reads $d \widetilde{s}:=e_{a} \vartheta^{a}=e_{\mu} \otimes \vartheta^{\mu} \in V_{4}$, and the holonomic metric on the space $V_{4}$ can be recast into the form $g=o_{a b} \vartheta^{a} \otimes \vartheta^{a}=g_{\mu \nu} \vartheta^{\mu} \otimes \vartheta^{\nu}$, with the components $g_{\mu \nu}=g\left(e_{\mu}, e_{\nu}\right)$ in dual holonomic basis $\left\{\vartheta^{\mu} \equiv d \widetilde{x}^{\mu}\right\}$, and $o_{a b}$ denotes $\operatorname{diag}(+---)$ metric. In the case at hand, the metric function $\omega$ is the angular velocity of the local ZAMO with respect to an observer at rest at infinity. Thereby the redshift factor $\alpha \equiv \exp \nu$ is the time dilation factor between the proper time of the local ZAMO and coordinate time $t$ along a radial coordinate line, i.e. the redshift factor for the time-slicing of a spacetime.

Given a height-function $\widetilde{t}$, the time-like unit normal to $\Sigma_{t}$ will be denoted by $n^{\mu}$ and the $3+1$ decomposition of the evolution vector field by $\widetilde{t}^{\mu}=N n^{\mu}+\beta^{\mu}$, where $N$ is the lapse function and $\beta^{\mu}$ is the shift vector. The induced metric on the space-like 3-slice $\Sigma_{t}$ is expressed as $\gamma_{\mu \nu}=g_{\mu \nu}+n_{\mu} n_{\nu}$, with $D_{\mu}$ the associated Levi-Civita connection and volume element ${ }^{3} \epsilon=\sqrt{\gamma} d \widetilde{x}^{1} \wedge d \widetilde{x}^{2} \wedge d \widetilde{x}^{3}$, so that ${ }^{3} \epsilon_{\mu \nu \rho}=n^{\sigma 4} \epsilon_{\sigma \mu \nu \rho}$. The extrinsic curvature of $\left(\Sigma_{t}, \gamma_{\mu \nu}\right)$ in $V_{4}$ reads $K_{\mu \nu}:=-(1 / 2) \mathcal{L}_{n} \gamma_{\mu \nu}=-\gamma_{\mu}{ }^{\rho} \nabla_{\rho} n_{\nu}$, where $\mathcal{L}$ denotes Lie derivative. In accord, all the geometrical objects are split into corresponding components with respect to this time-slice of spacetime.

In particular, the splitting of manifold $V_{4}$ into a foliation of three-surfaces will induce a corresponding splitting of the affine connection, curvature and, thus, of the energy-momentum tensor. The $3+1$ decomposition of the (matter) stress-energy tensor, measured by an adapted Eulerian observer of four-velocity $n^{\mu}$ in rest with respect to the foliation $\left\{\Sigma_{t}\right\}$, is $\widetilde{T}_{\mu \nu}=\widetilde{E} n_{\mu} n_{\nu}+\widetilde{p}_{(\mu} n_{\nu)}+\widetilde{S}_{\mu \nu}$, where the matter energy and momentum densities are given by $\widetilde{E}:=\widetilde{T}_{\mu \nu} n^{\mu} n^{\nu}$ and $\widetilde{p}_{\mu}:=-\widetilde{T}_{\nu \rho} n^{\nu} \gamma_{\mu}^{\rho}$, respectively, whereas the matter stress tensor is $\widetilde{S}_{\mu \nu}:=\widetilde{T}_{\rho \sigma} \gamma^{\rho}{ }_{\mu} \gamma^{\sigma}{ }_{\nu}$. Latin indices running in $\{1,2,3\}$ will be employed in expressions only involving objects intrinsic to space-like $\Sigma_{t}$ slices. That is, $\widetilde{T}^{\alpha \beta}=\widetilde{E} n^{\alpha} n^{\beta}+n^{\alpha} \widetilde{J}^{\beta}+\widetilde{J}^{\alpha} n^{\beta}+\widetilde{S}^{\alpha \beta}$. Here $n^{\alpha}$ is the unit orthogonal vector to the hypersurface $\Sigma_{t}$, whereas the spacetime metric $g$ induces a first fundamental form with the spatial metric $\gamma_{\alpha \beta}$ on each $\Sigma_{t}$ as $\gamma_{\alpha \beta}=g_{\alpha \beta}+n_{\alpha} n_{\beta}$. This form includes one gauge freedom for the coordinate choice. For the spherical type coordinates $\widetilde{x}^{2}=\widetilde{\theta}$ and $\widetilde{x}^{3}=\widetilde{r}$, for example, so-called quasi-isotropic gauge corresponds to $\gamma_{r \theta}=0$ and $\gamma_{\theta \theta}=\widetilde{r}^{2} \gamma_{r r}$. Then, one may define the second fundamental form which associates with each vector tangent to $\Sigma_{t}$, and the extrinsic curvature of the hypersur- 
face $\Sigma_{t}$ as minus the second fundamental form. Aftermath, one can define the usual Lorentz factor $W=-n_{\mu} \widetilde{u}^{\nu}=\alpha \widetilde{u}^{t}$ for a fluid which is the source of the gravitational field, with conventional stressenergy tensor $\widetilde{T}^{\mu \nu}=(\widetilde{\rho}+\widetilde{P}) \widetilde{u}^{\mu} \widetilde{u}^{\nu}+\widetilde{P} g^{\mu \nu}$, where $\widetilde{\rho}$ is the total energy density and $\widetilde{P}$ is the pressure. Hence $\widetilde{E}=W^{2}(\widetilde{\rho}+\widetilde{P})-\widetilde{P}$ and $\widetilde{J}^{i}=(\widetilde{E}+\widetilde{P}) \widetilde{v}^{i}$, where the fluid three-velocity $\widetilde{v}^{i}(i=1,2,3)$ implies $\widetilde{u}^{i}=W\left(\widetilde{v}^{i}-\beta^{i} / \alpha\right)$. Thereby the resulting stress tensor can be written $\widetilde{S}_{i j}=(\widetilde{E}+\widetilde{P}) \widetilde{v}_{i} \widetilde{v}_{j}+\widetilde{P} \gamma_{i j}$. The four-velocity for rotating fluid reads $\widetilde{u}=\widetilde{u}^{i}(\partial / \partial \widetilde{t})+\Omega \partial / \partial \widetilde{\phi}$, where $\Omega=\widetilde{u}^{\phi} / \widetilde{u}^{t}$ is the fluid angular velocity as seen by an inertial observer at rest at infinity.

Consequently, the components of the energy - momentum tensor of matter with total density $\rho$ and pressure $P$ are given in the non-rotating anholonomic orthonormal frame as $\widetilde{T}^{(a b)}=e_{\mu}^{a} e_{\nu}^{b} \widetilde{T}^{\mu \nu}, \quad \widetilde{T}^{(00)}=$ $W^{2}\left(\widetilde{\rho}+\widetilde{P} V^{2}\right), \quad \widetilde{T}^{(11)}=W^{2}\left(\widetilde{\rho}+\widetilde{P} V^{2}\right), \quad \widetilde{T}^{(01)}=W^{2}(\widetilde{\rho}+\widetilde{P}) V$ and $\widetilde{T}^{(22)}=\widetilde{T}^{(33)}=\widetilde{P}$, with its trace $\widetilde{T}=-\widetilde{\rho}+3 \widetilde{P}$, where $V$ is the velocity (in units of c) with respect to the Bardeen observer $V=\varrho B(\Omega-\omega) / \alpha^{2}$, so $W=1 / \sqrt{1-V^{2}}$.

The other physical features of SIMBH configuration also need to be accounted which comprise the whole of the case. The fact that the rotational energy has a steeper dependence on the radius of the compact object than the internal energy in the relativistic limit is quite significant. Equilibrium can always be achieved for massive configurations with nonzero angular momentum by decreasing its radius. Also, there are two characteristic features that distinguish a spinning relativistic SPCconfiguration from its non-spinning counterpart: 3) The geodetic effect, as in case of a gyroscope, leads an accretion stream to a tilting of its spin axis in the plain of the orbit. Hence a proto-matter disk will be tilted from the plane of accretion on a definite angle $\delta$ towards the equator. 4) Besides the UHE neutrinos, produced in the deep interior layers of superdense proto-matter medium as in case of non-spinning model, the additional thermal defuse blackbody radiation is released from the outer surface layers of ordinary matter of spinning SPC and co-spinning proto-matter thin disk. All of the rotational kinetic energy is dissipated as thermal blackbody radiation. This is due to the physical condition that these layers optically thick and, eventually, in earlier half of the lifetime of spinning black hole, at times $<T_{B H}$, the strict thermodynamic equilibrium prevails for this radiation because there would be no net flux to outside of event horizon in any direction. That is, the emission from the isothermal, optically thick outer layers at surface is blackbody, which is the most efficient radiation mechanism. This radiation is free of trapping. All of the rotational energy of SIMBH is dissipated as thermal defuse blackbody X-ray radiation to outside world.

\subsection{The X-ray radiation from SIMBH}

If this were the case, eventually the spinning proto-matter core and a thin co-spinning protomatter disk driven by accretion would be formed. The evolution and structure of a proto-matter disk is largely determined by internal friction. Before tempting to build a physical model in quest, the other features of SIMBH configuration also need to be accounted which comprise the whole of the case. The fact that the rotational energy has a steeper dependence on the radius of the compact object than the internal energy in the relativistic limit is quite significant. Equilibrium can always be achieved for massive configurations with nonzero angular momentum by decreasing its radius. Also, there are two characteristic features that distinguish a spinning relativistic SPC-configuration from its non-spinning counterpart: 3) The geodetic effect, as in case of a gyroscope, leads an accretion stream to a tilting of its spin axis in the plain of the orbit. Hence a proto-matter disk will be tilted from the plane of accretion on a definite angle $\delta$ towards the equator. 4) Besides the UHE neutrinos, produced in the deep interior layers of superdense proto-matter medium as in case of non-spinning model, the additional thermal defuse blackbody radiation is released from the outer surface layers of ordinary matter of spinning SPC and co-spinning proto-matter thin disk. All of the rotational kinetic energy is dissipated as thermal blackbody radiation. This is due to the physical condition that these layers optically thick and, eventually, in earlier half of the lifetime of spinning black hole, at times $<T_{B H}$, the strict thermodynamic equilibrium prevails for this radiation because there would be no net flux to outside of event horizon in any direction. That is, the emission from the isothermal, optically thick outer layers at surface is blackbody, which is the most efficient radiation mechanism. This radiation is free of trapping. With this guidelines to follow, we may proceed in relatively simple 


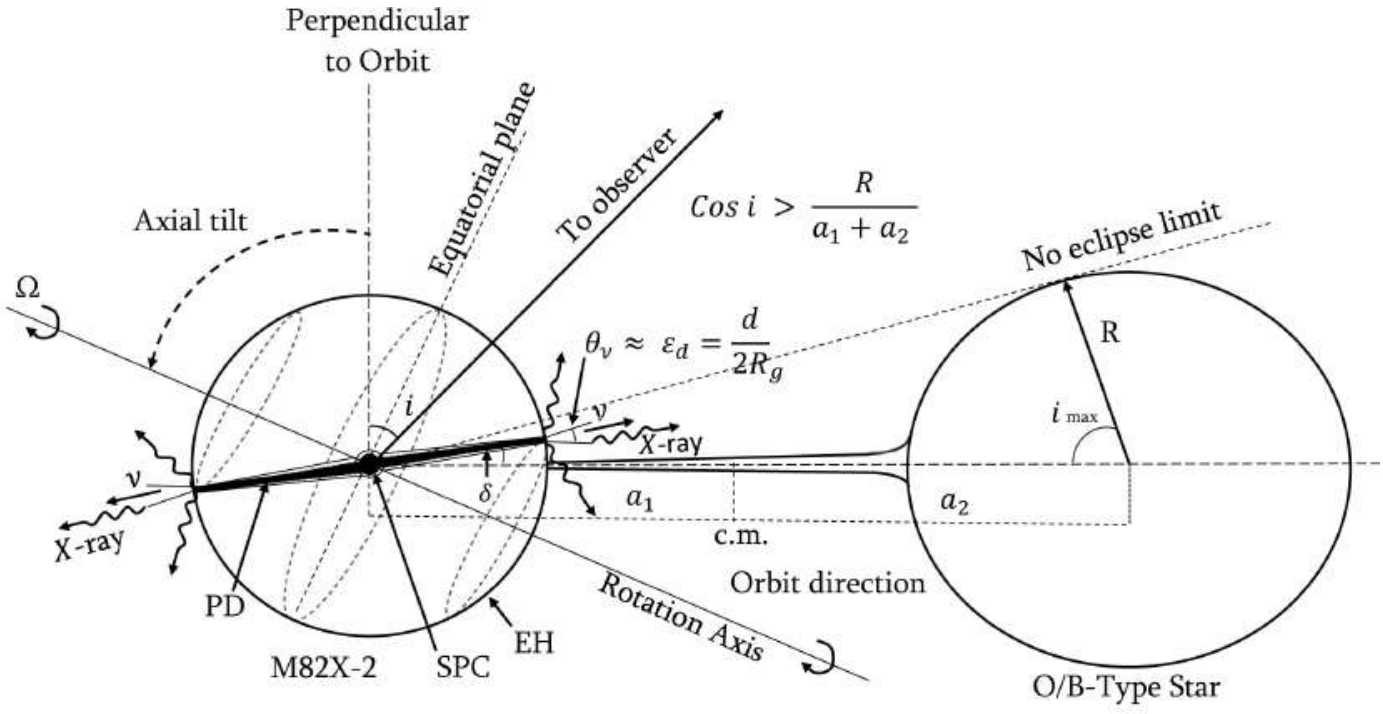

Figure 7. A schematic SIMBH model of M82X-2 constituting mass-exchange binary with the O/Btype donor star. The angle $i$ is the binary inclination with respect to the plane of the sky. No eclipse condition holds. In final stage of growth, PD has reached out the edge of the EH. The thermal defuse blackbody X-rays beams may escape from SIMBH through a thin belt area $S=2 \pi R_{g} d$ to outside world that sweep past Earth like lighthouse beacons. Parameters of a binary system is viewed in the orbital plane. The picture is not to scale. Accepted notations: $\mathrm{EH}=$ Event Horizon, SPC=Superdense Proto-matter Core, $\mathrm{PD}=$ Proto-matter Disk.

way toward first look at some of the associated physics and can be quick to estimate the physical characteristics of mass-exchange X-ray binaries. Examining the pulsations revealed from M82X-2, as a working model we assume the source of the flashes to be a self-gravitating SIMBH resided in the final stage of growth. This implies that a thin co-spinning proto-matter disk has reached out the edge of the event horizon. Due to it a metric singularity inevitably disappears at the intersection of proto-matter disk with the event horizon. Hence, the crossing event horizon at such conditions from inside of black hole is allowed. This general behavior is very robust and depends very little on the details of the model of SPC. A principle physical properties of this phenomena for non-rotating SPC are already discussed. Without being carefully treated, even though these properties are of great significant as well for a rotating SPCs. Actually, they will retain for a more general geometry of rotating axisymmetric SPCs. We conclude on the basis of these observations that the energy can be carried away to outside world by the thermal defuse blackbody X-ray radiation through a thin belt area $S$. As M82X-2 spins, the pulse profile results from the axial tilt or obliquity. Hence the X-ray beams intercept Earth-like lighthouse beacons. The orbital motion causes a modulation in the observed pulse frequency. The SIMBH model of M82X-2 in binary system is schematically plotted in Fig. 7. No eclipse condition holds. The parameters of a binary system is viewed in the orbital plane. The Fig. 8 explains the "spherical triangle". No eclipse condition holds. The parameters of a binary system is viewed in the orbital plane.

\subsection{Basic geometry: Implications on the pulse profile and mass scaling}

To see where all this is leading to, let us consider next the real issue that of the physical description of M82X-2. A knowledge of the dynamical mass measurements of the compact objects that power ULXs is a primarily necessary prerequisite to the derivation of a complete picture about the physical nature of ULXs. Keeping in mind aforesaid, we are now in a position to derive a general pulse profile dependent upon the position angles, and give a quantitative account of a potential dynamical mass scaling of M82X-2 and other energetics. The most reliable method is to measure the mass function through the secondary mass and orbital parameters, which can be measured only if the secondary donor star is optically identified. In the absence of direct mass-function measurements from phase- 


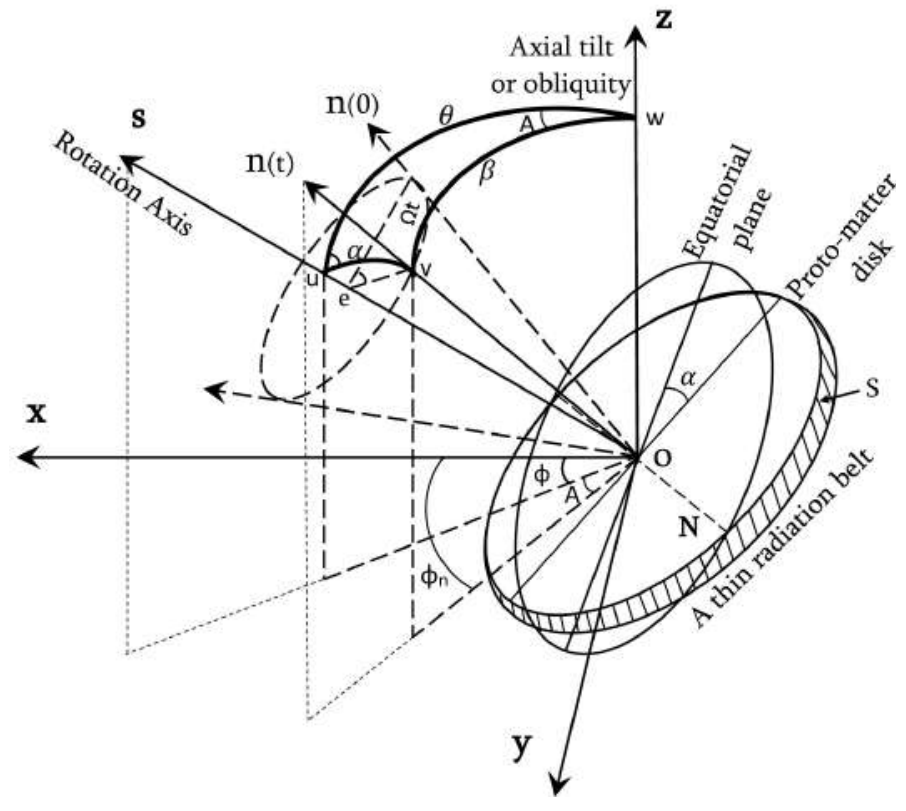

Figure 8. A schematic plot explaining the spherical triangle solved by the law of cosines. The spacefixed Cartesian coordinate system is labeled $(\mathrm{z}, \mathrm{x}, \mathrm{y})$, with zx as a plane-of sight. Axis $s$ of the M82X2 -fixed frame is rotation axis. The angles $\theta$ and $\phi$ are spherical polar coordinates. The line of nodes $\mathrm{N}$ is defined as the intersection of the equatorial and proto-matter disk planes. It is perpendicular to both the $\mathrm{z}$ axis and vector $\vec{n}(t)$, where $\vec{n}(t)$ is the normal to the plane of proto-matter disk at moment $t$. The $\vec{n}(0)$ lies in the plane of zs. The lengths of three sides of a "spherical triangle" (shown at top) are $\theta=\widehat{(z, s)}, \alpha=\widehat{(s, n)}$ and $\beta=\widehat{(z, n)}$. The vertex angle of opposite $\beta$ is $\Omega t$.

resolved optical spectroscopy, we still have to rely on X-ray spectral and timing modeling and other indirect clues. To bring this point sharply into focus note that in case of the first ultraluminous pulsar, only the X-ray mass function is measured, when the optical secondary is unknown and most of the orbital parameters are yet to be measured. However, exploring the key physical characteristics of a SIMBH model, let us consider the space-fixed Cartesian coordinate system labeled $(\mathrm{z}, \mathrm{x}, \mathrm{y})$, with zx as a plane-of sight, and the axis $s$ of the M82X-2-fixed frame as the rotation axis. A schematic plot is given in Fig. 8. Here and throughout we now use following notational conventions. The angles $\theta$ and $\phi$ are spherical polar coordinates. The observed pulses are produced because of periodic variations with time of the projection $d_{z x}(t)$ of the vector $\vec{d}(t)$ aligned with the $\vec{n}(t)\left(\vec{d}(t)=d \frac{\vec{n}(t)}{|n(t)|}\right)$ on the plane-of sight, where $\vec{n}(t)$ is the normal to the plane of the proto-matter disk at the moment $t$. The $\vec{n}(0)$ lies in the plane of zs. So, these pulsations are due to the fact that the visible surface is less at one moment than at another. The Fig. 8 explains the spherical triangle. That is, given a unit sphere, a spherical triangle on the surface of the sphere is defined by the great circles connecting three points $\mathrm{u}, \mathrm{v}$, and $\mathrm{w}$ on the sphere (shown at top). The lengths of these three sides are $\alpha=\widehat{(s, n)}$ (from $\mathrm{u}$ to $\mathrm{v}$ ), the axial tilt $\theta=\widehat{(z, s)}$ (from $\mathrm{u}$ to $\mathrm{w}$ ), and $\beta=\widehat{(z, n)}$ (from $\mathrm{v}$ to $\mathrm{w}$ ). The angles of the corners $u$ and $e$ opposite $\beta$ equal $u=e=\Omega t$. The proto-matter disk was shifted from the orbital direction on angle $\delta=\theta-\alpha$ towards the equator. The projection $d_{z x}(t)$ is written $d_{z x}(d, \theta, \phi, \alpha, t)=\sqrt{d_{z}^{2}(d, \theta, \alpha, t)+d_{x}^{2}(d, \theta, \alpha, \phi, t)}$, where $d_{z}(d, \theta, \alpha, t) \equiv \vec{d}(\ldots, t) \cdot \overrightarrow{e_{z}}=d \cos \beta(\theta, \alpha, t)$, and $d_{x}(d, \theta, \alpha, \phi, t) \equiv \vec{d}(\ldots, t) \cdot \overrightarrow{e_{x}}=d \sin \beta(\theta, \alpha, t) \cos \phi_{n}(\theta, \alpha, \phi, t)$. Here the $\vec{e}_{z}$ and $\vec{e}_{x}$ denote unit vectors along the axes $z$ and $x$, respectively, $\phi_{n}=\phi+A$ is the azimuthal angle of vector $\vec{n}(t)$. The vertex angle opposite the side $\alpha$ is $A$. To the extent that all of the rotational energy of M82X-2 is dissipated as thermal defuse X-ray blackbody radiation, this may escape from the event horizon to outside world only through a thin belt area $S$. The radiation arisen from per area of surface is $\sigma T_{s}^{4}$, where $T_{s}$ is the surface temperature, $\sigma$ is the Stefan-Boltzmann constant. Therefore, the pulsed luminosity $\widetilde{L}$ will be observed if and only if the projection of the belt area $S_{z x}=2 \pi R_{g} d_{z x}(d, \theta, \phi, \alpha, t)$ on the plane-of sight zx is not zero. So, pulsed luminosity reads $\widetilde{L}\left(R_{g}, d, T_{s}, \theta, \phi, \alpha, t\right)=S_{z x} \sigma T_{s}^{4}=$ 


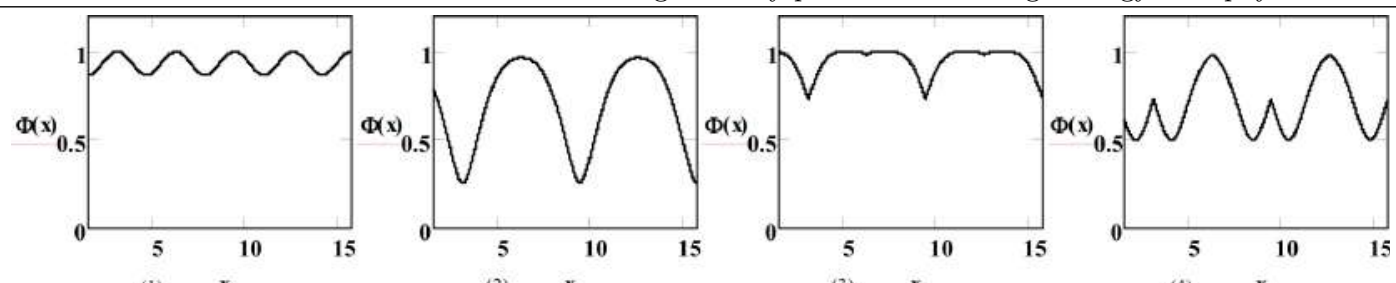

(1) $\mathbf{x} \quad$ (2) $\mathbf{x} \quad$ (3) $\mathbf{x} \quad$ (4) $\mathbf{x}$
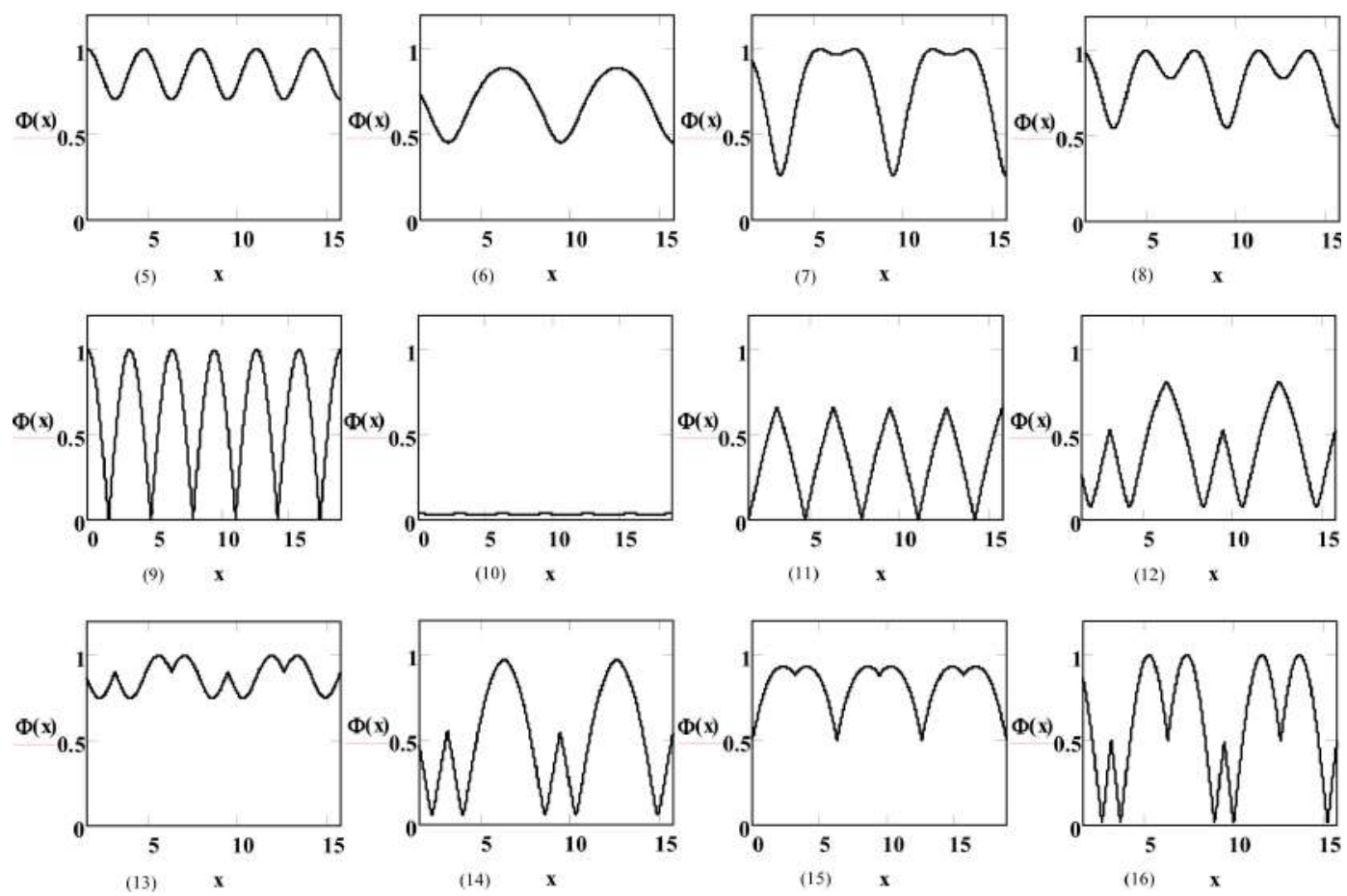

Figure 9. Characteristic phase profiles versus the time $(x \equiv \Omega t)$, viewed at the following position angles $(\theta, \alpha, \phi)$ : (1) $\left(45^{0}, 30^{0}, 0^{0}\right)$; (2) $\left(45^{0}, 30^{0}, 90^{0}\right)$; (3) $\left(45^{0}, 30^{0}, 135^{0}\right)$; (4) $\left(45^{0}, 30^{0}, 60^{0}\right)$; (5) $\left(45^{0}, 90^{0}, 90^{0}\right)$; (6) $\left(45^{0}, 18^{0}, 90^{0}\right)$; (7) $\left(45^{0}, 60^{0}, 90^{0}\right)$; (8) $\left(45^{0}, 78.3^{0}, 90^{0}\right)$; $\quad$ (9) $\left(90^{0}, 90^{0}, 180^{0}\right)$; (10) $\left(90^{0}, 0.6^{0}, 72^{0}\right)$; (11) $\left(90^{0}, 30^{0}, 60^{0}\right)$; (12) $\left(72^{0}, 30^{0}, 60^{0}\right)$; (13) $\left(60^{0}, 90^{0}, 60^{0}\right)$; (14) $\left(60^{0}, 45^{0}, 60^{0}\right)$; (15) $\left(60^{0}, 153^{0}, 60^{0}\right) ;(16)\left(1.2^{0}, 90^{0}, 60^{0}\right)$.

$2 \pi R_{g} d_{z x}(d, \theta, \phi, \alpha, t) \sigma T_{s}^{4} \equiv L_{0}\left(M, d, T_{s}\right) \Phi(\theta, \phi, \alpha, t)$, where its amplitude and phase, respectively, are $L_{0}\left(M, d, T_{s}\right) \simeq 1.05 \times 10^{4}\left(\operatorname{erg~s}^{-1}\right) \frac{M}{M_{\odot}} \frac{d}{\mathrm{~m}} \frac{T_{s}^{4}}{K^{4}}, \Phi(\theta, \phi, \alpha, t) \equiv \sqrt{1-\sin ^{2} \beta \sin ^{2}(\phi+A)}$. The spherical triangle is solved by the law of cosines $\cos \beta(\theta, \alpha, t)=\cos \theta \cos \alpha+\sin \theta \sin \alpha \cos \Omega t, \cos A(\theta, \alpha, t)=$ $\frac{\cos \alpha-\cos \theta \cos \beta}{\sin \theta \sin \beta}$.

Consequently, the pulsed flux can be written in the form $\widetilde{F}\left(R_{g}, d, \theta, \phi, \alpha, t\right)=F_{0}(M, d) \Phi(\theta, \phi, \alpha, t)$. where, given the distance $D \simeq 3.6 \mathrm{Mpc}$ to the galaxy M82, the flux amplitude is $F_{0}(M, d)=\frac{L_{0}(M, d)}{4 \pi D^{2}} \simeq$ $6.8 \times 10^{-48}\left(\mathrm{erg} \mathrm{s}^{-1} \mathrm{~cm}^{-2}\right) \frac{M}{M_{\odot}} \frac{d}{\mathrm{~m}} \frac{T_{s}^{4}}{K^{4}}$. Thus, the theoretical model of periodic source M82X-2 left six free parameters: $\left(M, d, T_{s}, \theta, \phi, \alpha\right)$. The Fig. 9 reveals the diversity of the behavior of characteristic phase $\Phi(\theta, \phi, \alpha, x \equiv \Omega t)$ profiles versus the time, viewed at given position angles $(\theta, \phi, \alpha)$. The observed X-ray pulse is further determined by the complicated transfer of X-ray photons from the surface of M82X-2 through regions of external accreting plasma. At hard look, the position angles being the parameters of a model function can be adjusted via nonlinear regression analysis to the approximate solution of overdetermined systems to best fit a data set from observed pulsed profile of M82X-2. These missing ingredients are the shortcoming of present framework, which will be solved by iterative refinement elsewhere. For maximum value of pulsed luminosity either at $\beta=\pi s$ or $\phi+A=\pi s(\mathrm{~s}=0,1,2, \ldots)$, we have $L_{0}\left(M, d, T_{s}\right)=\widetilde{L}(3-30 \mathrm{keV})=4.9 \times 10^{39} \operatorname{erg~s}^{-1}$ or $F_{0}(M, d)=$ $\widetilde{F}(3-30 \mathrm{keV}) \simeq 3.16 \times 10^{-12} \mathrm{erg} \mathrm{s}^{-1} \mathrm{~cm}^{-2}$. Hence the surface temperature scales $\propto T_{s}^{-4}$ with the black hole mass: $\frac{M}{M_{\odot}}=\frac{4.66 \times 10^{35} K^{4}}{T_{s}^{4}} \frac{\mathrm{m}}{d}$. That is, a cooler radiation surface implies a bigger black hole. If we further assume that the persistent emission $-\bar{L}(0.3-10 \mathrm{keV})=1.8 \times 10^{40} \mathrm{erg} \mathrm{s}^{-1}$ - from M82X-2 
Ambartsumian's vision and further insight to key puzzles of ultra-high energy astrophysics

is isotropic, we may impose a strict Eddington limit on the mass transfer rate that can be accepted by the black hole, $\bar{L}<L_{E d d} \simeq 1.3 \times 10^{38} \frac{M}{M_{\odot}} \mathrm{erg} \mathrm{s}^{-1}$. This imposes stringent constraint on the lower limit of black hole mass $\frac{M}{M_{\odot}}>138.5, \quad R_{g}>408.6 \mathrm{~km}$, because for an accreting of $\sim 10$ per cent of the Eddington limit - a fairly typical accretion rate for a high-state black hole - this points towards a rough estimate of upper limit of mass $M<1385 M_{\odot}$.

\subsection{More accurate determination of upper mass limit of M82X-2}

For the knowledge of upper mass limit of M82X-2, a good progress can still be made by establishing a direct physical relation between masses of M82X-2 and M82X-1, and then one should rely on the available mass estimates of the latter (Ter-Kazarian, 2016b). The controversy, however, is with their mass range. As one may envisage, a different mass estimates of M82X-1 may yield different mass values of M82X-2. So, we should be careful in choosing the most accurate black hole mass measurement todate. As the centroid of the persistent emission is between M82X-2 and M82X-1 which indicates that M82X-2 harbors an IMBH, likewise M82X-1, we suppose that accretion onto a black hole is well approximated by the relation $L_{a c c}=\eta c^{2} \dot{M}=\frac{G M}{R} \dot{M}$. This gives $\dot{M}_{1} / \dot{M}=\bar{L}_{1} / \bar{L} \simeq 5.556$. According to $\mathrm{MTBH}$, we have $\dot{M} \propto M^{2}$ for both the collisionless and the hydrodynamic spherical accretions onto black hole. Making use of these relations, we then obtain $M_{1} \simeq 2.357 M$. The M82X-1 is a good candidate for hard state ULXs which may be one of the very few ULXs that change their spectral state during outbursts, switching from a hard to a thermal state. The "type-C" low frequency quasi-periodic oscillations and broadband timing noise, detected in the two XMM-Newton observations in 2001 and 2004, in the central region of M82 and later confirmed to originate from the M82X-1, suggest that the ULX harbors a massive black hole. The used mass estimate is based on the assumption that M82X-1 also follows well established relation of the photon spectral index versus QPO frequency, $\Gamma-\nu_{Q P O}$, found for the Galactic X-ray binaries in their high or intermediate states. The resulting IMBH mass for M82X-1 is in the range of $25-520 M_{\odot}$. However, there may be systematic errors in the photon indices measured with XMM-Newton and Rossi X-ray Timing Explorer (RXTE) due to contamination from nearby sources, as indicated by large apparent changes in the effective absorption column. Another mass estimate is inferred from the correlations with the X-ray luminosity and type C QPO frequency. This method is based on the correlation between characteristic frequencies, on the fundamental plane and on the variability plane of accreting black holes. Exploring this method, the black hole masses inferred from the characteristic frequencies are all about $10^{3}-10^{4} M_{\odot}$ indicating that ULXs contain IMBHs. But, these results were not without problems, notably pointed out by the same authors. Such mass estimates are based on scaling relations which use low-frequency characteristic timescales which have large intrinsic uncertainties. In particular, it was unclear whether these $\mathrm{mHz}$ oscillations are indeed the Type-C analogs of stellar mass black, and both the Type-C and the $\mathrm{mHz}$ oscillations are variable, resulting in a large dispersion in the measured mass of $25-1300 M_{\odot}$. Positive identification of the emission states requires both timing and spectral information. Consequently, with simultaneous observations exploiting the high angular resolution of Chandra to isolate the ULX spectrum from diffuse emission and nearby sources and the large collecting area of XMM-Newton observations of M82 to obtain timing information, it is surprisingly found that the previously known QPOs in the source disappeared. The light curve was no longer highly variable and the power spectrum was consistent with that of white noise. The energy spectrum also changed dramatically from a straight power-law to a disk-like spectrum. The disappearance of QPOs and the low noise level suggest that the source was not in the hard state. All results are well consistent with that expected for the thermal state. The monitoring data from RXTE indicate that these Chandra and XMM-Newton observations were made during the source outbursts, suggesting that M82X-1 usually stays in the hard state and could transition to the thermal state during outbursts. The spectral fitting suggests that the ULX contains a close to Eddington $\left(L_{d i s k} / L_{E d d} \sim 2\right)$ rapidly spinning IMBH of $200-800 M_{\odot}$ masses. The thermal dominant states are all found during outbursts. Nonetheless, modeling of X-ray energy spectra during the thermal-dominant state using a fully relativistic multi-colored disk model has large uncertainties owing to both systematic and measurement errors. In addition to the large mass uncertainty associated with the modeling, the same authors also found that the energy spectra can be equally well-fit with a stellar-mass black hole accreting at a rate of roughly 160 higher than the Eddington limit. Also, the 
X-rays from this source are known to modulate with an orbital periodicity of 62 days, which indicates to an intermediate-mass black hole with mass in the range of $200-5000 M_{\odot}$. But, a recent study finds that this periodicity may instead be due to a precessing accretion disk in which case a stellarmass black hole will suffice to explain the apparent long periodicity. Thus, the mass measurements above have large uncertainties. This makes black hole masses obtained by this method at the very least questionable. In what follows, therefore, we adopt an alternative and less ambiguous mass determination for intermediate-mass black holes, which seems to be a more reliable determinant of the mass of M82X-1. The searched was made of RXTE's proportional counter array archival data to look for $3: 2$ oscillation pairs in the frequency range of $1-16 \mathrm{~Hz}$ which corresponds to a black hole mass range of $50-2000 M_{\odot}$. In stellar-mass black holes, it is known that the high frequency quasi-periodic oscillations that occur in a $3: 2$ ratio $(100-450 \mathrm{~Hz})$ are stable and scale inversely with black hole mass with a reasonably small dispersion. It is reported of stable, twin-peak ( $3: 2$ frequency ratio) X-ray quasi-periodic oscillations from M82X-1 at the frequencies of $3.32 \pm 0.06 \mathrm{~Hz}$ (coherence, Q $=$ centroid frequency $(\nu) / \operatorname{width}(\nu)>27)$ and $5.07 \pm 0.06 \mathrm{~Hz}(Q>40)$. The discovery of a stable $3: 2$ high-frequency periodicity simultaneously with the low-frequency $\mathrm{mHz}$ oscillations allows for the first time to set the overall frequency scale of the X-ray power spectrum. This result not only asserts that the $\mathrm{mHz}$ quasi-periodic oscillations of $\mathrm{M} 82 \mathrm{X}-1$ are the Type-C analogs of stellar-mass black holes but also provides an independent and the most accurate black hole mass measurement to-date. Assuming that one can scale the stellar-mass relationship, they estimate the black hole mass of M82X-1 to be $428 \pm 105 M_{\odot}$. They also estimate the mass using the relativistic precession model, which yields a value of $415 \pm 63 M_{\odot}$. Combining the average $2-10 \mathrm{keV}$ X-ray luminosity of the source of $5 \times 10^{40} \mathrm{ergs} \mathrm{s}^{-1}$ with the measured mass suggests that the source is accreting close to the Eddington limit with an accretion efficiency of $0.8 \pm 0.2$.

Making use of the mass values $428 \pm 105 M_{\odot}$, we provide the mass estimate for M82X-2: $M \simeq$ $138.5-226 M_{\odot}, \quad R_{g} \simeq 408.6-666.7 \mathrm{~km}$, Rotation speed at surface of M82X-2 with upper limit mass $226 M_{\odot}$ as rigidly spinning IMBH configuration of angular velocity $\Omega$ equals $v=R_{g} \Omega \simeq 3.06 \times$ $10^{8} \mathrm{~cm} \mathrm{~s}^{-1}$. We may also slightly improve the lower mass limit $323 M_{\odot}$ of M82X-1, to now be $326.5 M_{\odot}$. Combining all the results above, we obtain then $2.06 \times 10^{33}<\frac{T_{s}^{4}}{K^{4}} \frac{d}{\mathrm{~m}}<3.34 \times 10^{33}$. For reasons that will become clear below, next, in our setting we adopt the rather concrete proposal of non-spinning black holes, i.e. the neutrino flux from spinning M82X-2 might as well be highly beamed along the plane of proto-matter disk and collimated in very small opening angle. For the adopted values, this yields the constraints $7.5 \times 10^{-7} \frac{d}{\mathrm{~m}}<\theta_{\nu} \sim \varepsilon_{d}<1.2 \times 10^{-6} \frac{d}{\mathrm{~m}}$. Besides, the $\varepsilon_{d}$ is likely to be about an order of magnitude $\sim 10^{-5}$ for M82X-2. Therefore, $d \simeq 61-100 \mathrm{~m}, \quad \varepsilon_{d} \simeq(4.6-7.5) \times 10^{-5}$, is a good guess for the thickness of proto-matter disk at the edge of event horizon. This gives $T_{s} \simeq 7.6 \times 10^{7} \mathrm{~K}$. Thus, M82X-2 indeed releases $\sim 99.6 \%$ of its radiative energy predominantly in the X-ray bandpass of $0.3-30$ $\mathrm{keV}$. However, its studies in other wavelengths well give us useful information on its physical nature and environment. From Wien's displacement law we obtain the wavelength $\lambda_{\max } \simeq 0.381 \mathrm{~nm}$ at which the radiation curve peaks, which corresponds to energy $h \nu_{\max } \simeq 3.2 \mathrm{keV}$. As an immediate corollary to the assumption that the emission arisen from accretion is isotropic, we are able to infer the most important ratios of the pulsed and persistent luminosities to the isotropic Eddington limit for M82X2: $\frac{\widetilde{L}}{L_{E d d}} \simeq 0.17-0.28, \frac{\bar{L}}{L_{E d d}} \simeq 0.63-1.03$, respectively, where $L_{E d d} \simeq(1.75-2.85) \times 10^{40} \mathrm{erg} \mathrm{s}^{-1}$. These properties appear consistent with the sub-Eddington hard state, which given the observed luminosities of M82X-2 suggests the presence of SIMBH with a dynamical mass. Given the angular velocity $\Omega=\frac{2 \pi}{P}(P=1.37 \mathrm{~s})$, we may calculate the rotational kinetic energy $E_{\text {rot }}=\frac{1}{2} I \Omega^{2}$ of M $82 \mathrm{X}-2$, where $I=\frac{2}{5} M R_{g}^{2}$ is the moment of inertia if M82X-2 is regarded as the rigidly spinning canonical configuration of mass $M$ and radius $R_{g}$. Hence $E_{\text {rot }} \simeq(3.72-16.17) \times 10^{51} \mathrm{erg}$. When all energy thermalized, radiation emerges as a blackbody. A significant fraction of the accretor M82X-2 surface radiates the accretion luminosity at temperature $T_{b}=\left(\frac{L_{a c c}}{4 \pi R_{g}^{2}}\right)^{1 / 4} \simeq\left(\frac{L_{E d d}}{4 \pi R_{g}^{2}}\right)^{1 / 4}$, such that $T_{b} \simeq$ $(3.88-5.37) \times 10^{6} \mathrm{~K}$. The gravitational energy of each accreted electron-proton pair turned directly into heat at (shock) temperature $T_{s h}: 3 k T_{s h}=\frac{G M m_{p}}{R_{g}}$, so $T_{s h} \simeq 1.8 \times 10^{12} \mathrm{~K}$. Hence typical photon energies of persistent radiation lies between $k T_{b} \simeq 0.34 \mathrm{keV} \leq h \nu \leq k T_{s h} \simeq 180 \mathrm{MeV}$. So, M82X2 is persistent X-ray and possibly gamma-ray emitter. Also, given the mass of the most brightest 
source M82X-1 of persistent X-ray radiation, typical photon energies of persistent radiation lie in range $k T_{b(X 1)} \simeq 0.3 \mathrm{keV} \leq h \nu \leq k T_{s h} \simeq 180 \mathrm{MeV}$.

\subsection{The mass of companion star and orbit parameters}

Once the mass scaling of M82X-2 is accomplished, this can potentially be used further to quantify the association between the M82X-2 and the optical secondary donor star in X-ray binary. The orbital period $P_{o r}$ is a key parameter for dynamical mass measurement. From Fig. 7, the separation of the two masses is $a$, and their distances from the center of mass are $a_{1}$ and $a_{2}$. The highly circular orbit, combined with the mass function $f\left(M, M_{2}, i\right)=2.1 M_{\odot}$, the lack of eclipses and assumption of a Roche-lobe- filling companion constrain the inclination angle to be $i<60^{\circ}$. These alow to determine the mass $M_{2}$ of donor star: $\frac{M_{2}}{M_{\odot}}>\left\{\begin{array}{ll}48.3, & \text { for } M=138.5 M_{\odot}, \\ 64.9, & \text { for } M=226 M_{\odot} .\end{array}\right.$ Thus, optical companion is a typical O/B supergiant, which evolves away from the main sequence in just a few million years. The binary separation can be computed with Kepler's Law $a^{3}=\frac{G\left(M+M_{2}\right)}{4 \pi^{2}} P_{o r}^{2}$. The Doppler curve of the spectrum of NuSTAR J095551+6940.8 shows a $P_{o r}=2.5$-day sinusoidal modulation arisen from binary orbit. All these give the projection of the orbital velocity of M82X-2 along the line of sight $v_{1}=\frac{2 \pi}{P_{\text {or }}} a_{1} \sin i \simeq 200.9 \frac{\mathrm{km}}{\mathrm{s}}$, and hence $a_{1} \sin i \simeq 9.9 R_{\odot}$. The absence of eclipses implies $\cos i>\frac{R}{a}$, where $R$ is the radius of companion donor star. Hence $R>\left\{\begin{array}{ll}22.1 R_{\odot}, & \text { for } M=138.5 M_{\odot}, \\ 25.7 R_{\odot}, & \text { for } M=226 M_{\odot} .\end{array}\right.$ As well as $a_{1}>11.4 R_{\odot}, a_{2}>32.9 R_{\odot}$ for $M / M_{\odot}=138.5$; and $a_{1}$ is the same, $a_{2}>39.9 R_{\odot}$ for $M / M_{\odot}=226$. The Roche lobe radius $R_{L}$ for donor star is $R_{L}> \begin{cases}15 R_{\odot}, & \text { for } \quad M=138.5 M_{\odot}, \\ 14.3 R_{\odot}, & \text { for } \quad M=226 M_{\odot} .\end{cases}$ Thus, the M82X-2 and donor star constitute the semi-detached binary, accreting through Roche-lobe overflow. Donor star exceeds its Roche lobe $\left(R>R_{L}\right)$, therefore its shape is distorted because of mass transfer from donor star through the inner Lagrange point $L_{1}$ to the M82X-2. The accretion stream is expected to be rather narrow as it flows through the $L_{1}$ point and into the Roche lobe of the primary.

\subsection{Spin-up rate and the torque added to $\mathrm{M} 82 \mathrm{X}-2$}

All accreting pulsars show stochastic variations in their spin frequencies and luminosities, including those displaying secular spin-up or spin-down on long time scales, blurring the conventional distinction between disk-fed and wind-fed binaries. Pulsed flux and accretion torque are strongly correlated in outbursts of transient accreting pulsars, but uncorrelated, or even anticorrelated, in persistent sources. The observed secular spin-up rate can be accounted for quantitatively if one assumes the reduction of the torque on the rapidly spinning object. Continuing on our quest, below we determine the conditions under which pulsed source M82X-2 spins up and hance gains rotational energy as matter is accreted, i.e. we discuss the relationship between the properties of the exterior flow and the measured rate of change of angular velocity $d \Omega / d t$. Although measurements of spin-up or spin-down appears to be the most promising method for determining the angular momentum transport by the inflowing matter, which in turn, may provide information about pattern of material flow outside the event horizon of SIMBH, the extraction of this information from such measurements clearly requires some care. We explore the relationship between the torque $(\equiv l)$ flux through the event horizon, spin-up rate of SIMBH and the rate of change of its rotational energy. The rates of change of the SIMBH angular velocity and of the rotational energy can be related to the flux of torque across the event horizon boundary as follows. The rate of change of the torque is given by $\frac{d}{d t}(I \Omega)=\dot{M} l$, where $I$ is the moment of inertia, and $l$ is the torque added to the SIMBH per unit mass of accreted matter. This equation gives for the rate of change of angular velocity $\frac{d \Omega}{d t}=\frac{\dot{M}}{L_{b h}}\left[l \Omega-\Omega^{2} R_{\text {gir }}^{2}\left(\frac{M}{I} \frac{d I}{d M}\right)\right]$, where $L_{b h} \equiv I \Omega$, and $R_{g i r}$ is the radius of gyration of SIMBH. The rate of change of the rotational energy is $\frac{d E_{r o t}}{d t}=\frac{d}{d t}\left(\frac{1}{2} I \Omega^{2}\right)$. To make further progress we recast these equations into the form $\frac{d E_{\text {rot }}}{d t}=\dot{M}\left[l \Omega-\frac{1}{2} \Omega R_{\text {gir }}^{2}\left(\frac{M}{I} \frac{d I}{d M}\right)\right]$. When $\frac{M}{I} \frac{d I}{d M}>0$, which is generally the case, the SIMBH's behavior can be conveniently characterized by the dimensionless parameter $\zeta \equiv \frac{l}{\Omega R_{g i r}^{2}}\left(\frac{M}{I} \frac{d I}{d M}\right)^{-1}$. Thus the black hole loses rotational energy and spins down for $\zeta<1 / 2$, whereas it gains rotational energy and 
spins up for $\zeta>1$; for $1 / 2<\zeta<1$ the black hole spins down even though it is gaining rotational energy. The logarithmic derivative $\frac{M}{I} \frac{d I}{d M}$ for canonical configuration, i.e. spinning uniform-density sphere with mass $M$ and radius $R_{g}$, is $\frac{d \ln I}{d \ln M}=\frac{d}{d \ln M} \ln \left(\frac{2}{5} M R_{g}^{2}\right)=3$, so $\zeta=\frac{l}{3 \Omega R_{\text {gir }}^{2}}$, where $R_{\text {gir }}^{2}=$ $I / M=\frac{2}{5} R_{g}^{2}$. For the spin up regime of M82X-2 when $\zeta \simeq 0.073 \frac{l}{\Omega R_{g i r}^{2}}>1$, we obtain $l>2.192 R_{g}^{2} \mathrm{~s}^{-1}$, so $l>\left\{3.7 \times 10^{5} \mathrm{~km}^{2} \mathrm{~s}^{-1}, \quad\right.$ for $M=138.5 M_{\odot} 9.7 \times 10^{5} \mathrm{~km}^{2} \mathrm{~s}^{-1}, \quad$ for $M=226 M_{\odot}$. The time derivative of the angular velocity gives $l=\frac{4 \pi R_{g}^{2}}{5 P}\left(3-\frac{\dot{P}}{P} \frac{M}{\dot{M}}\right)$. Combing $\dot{M} \simeq 6.35 \times 10^{-7} M_{\odot} y r^{-1}$, and a linear spin-up $\dot{p}$ of the NuSTAR J095551+6940.8 pulsar, we obtain the torques added to M82X-2 per unit mass of accreted matter, which satisfy the spin-up condition of $\zeta>1$ : $l \simeq$ $\begin{cases}1.1 \times 10^{11} \mathrm{~km}^{2} \mathrm{~s}^{-1}, & \text { for } \quad M=138.5 M_{\odot}, \\ 9.8 \times 10^{11} \mathrm{~km}^{2} \mathrm{~s}^{-1}, & \text { for } \quad M=226 M_{\odot}\end{cases}$

\section{Rotating black holes in microscopic theory: the implications for periodic source M82X-2}

For rigorous theoretical solutions, we analytically treat the microscopic model of stationary and axisymmetric rotating black hole (Ter-Kazarian, 2016a). In particular, we derive field equations and obtain both internal and global vacuum spacetime solutions. A ring singularity of the Kerr black hole cannot occur, which is now replaced by equilibrium SPC. We calculate the corrections to previous model introduced by the rotation of the characteristic phase profile of M82X-2.

\subsection{Axisymmetric distortion of $M_{6} \rightarrow V_{6}:$ A reduction to $V_{4}$}

Before tempting to build an axisymmetric distorted Riemannian $4 \mathrm{D}$ space $V_{4}$, we need to consider an axisymmetric distortion of the $6 \mathrm{D}$ space $M_{6} \rightarrow V_{6}$, and next reduce it to $V_{4}$. The element $D(a, \theta)$ of the distortion group $G_{D}$ has induced a general distortion transformations of the six-basis vectors $e=O \times \sigma\left(\in M_{6}\right) \rightarrow \widetilde{e}=\widetilde{O} \times \widetilde{\sigma}\left(\in V_{6}\right): 2 \widetilde{e}_{(0 \alpha)}=\widetilde{\xi}_{(0 \alpha)} \times\left(\widetilde{\sigma}_{(+\alpha)}+\widetilde{\sigma}_{(-\alpha)}\right)+\widetilde{\xi}_{(\alpha)} \times\left(\widetilde{\sigma}_{(+\alpha)}-\widetilde{\sigma}_{(-\alpha)}\right), 2 \widetilde{e}_{(\alpha)}=$ $\widetilde{\xi}_{(\alpha)} \times\left(\widetilde{\sigma}_{(+\alpha)}+\widetilde{\sigma}_{(-\alpha)}\right)+\widetilde{\xi}_{(0 \alpha)} \times\left(\widetilde{\sigma}_{(+\alpha)}-\widetilde{\sigma}_{(-\alpha)}\right)$, where distortion transformations of the ingredient basis vectors $(O)$ and $(\sigma)$ are not independent. Hence $\delta \widetilde{\xi}_{(0 \alpha)}=-\xi_{0} x_{(0 \alpha)}+\xi x_{\alpha}+\bar{\xi}_{0} \bar{x}_{(0 \alpha)}-\bar{\xi} \bar{x}_{\alpha}, \delta \widetilde{\xi}_{\alpha}=$ $\xi x_{(0 \alpha)}-\xi_{0} x_{\alpha}+\bar{\xi}_{0} \bar{x}_{\alpha}-\bar{\xi} \bar{x}_{0 \alpha)}$, provided by $\delta \widetilde{\sigma}_{A}(\theta)=\delta \mathcal{R}_{A}^{\beta}(\theta) \sigma_{\beta}$. Let $\phi\left(=x^{1}\right)$ be the azimuthal angle about the axis of symmetry in $R^{3}$. The axisymmetric distortion of the space $M_{6} \rightarrow V_{6}$ can be induced by the distortion gauge field $a_{A}$, with the only non-zero $1 \mathrm{D}$ components of $x_{0}:=x_{(03)}=æ a_{( \pm 3)}$ and $x_{1}=\mp æ a_{( \pm 1)}$, in presence of ID-field, $\bar{x}:=\bar{x}_{(03)}=æ \bar{a}_{( \pm 3)}$, where a coupling constant $æ$ relates to the Newton's gravitational constant $G_{N}$. Then, the non-zero components of the transformation matrix $D$ can be recast into the form $D_{(\tilde{01})}^{(01)}=1, D_{(\tilde{01})}^{1}=x_{1}, D_{(\tilde{02})}^{(02)}=\cos \theta_{1}, D_{(\tilde{02})}^{3}=\sin \theta_{1}, D_{(\tilde{03})}^{(03)}=\cos \theta_{1}\left(1-x_{0}\right)$, $D_{(\tilde{03})}^{2}=-\sin \theta_{1}\left(1+x_{0}\right), D_{(\tilde{03})}^{\overline{3}}=-\cos \theta_{1}, \quad D_{(\tilde{03})}^{(\overline{02})}=-\sin \theta_{1}, D_{\tilde{1}}^{1}=1, D_{\tilde{1}}^{(01)}=-x_{1}, D_{\tilde{2}}^{2}=\cos \theta_{1}$, $D_{\tilde{2}}^{(03)}=\sin \theta_{1}, D_{\tilde{3}}^{3}=\cos \theta_{1}\left(1+x_{0}\right), D_{\tilde{3}}^{(02)}=-\sin \theta_{1}\left(1-x_{0}\right), D_{\tilde{3}}^{(\overline{03})}=\cos \theta_{1} x_{1}, D_{\tilde{3}}^{\overline{2}}=-\sin \theta_{1} x_{1}$, where the rotation angles are determined as $\pm \theta_{(1)}:=\tan \theta_{( \pm 1)}=\mp x_{1}, \tan \theta_{( \pm 3)}=x_{(0)}, \quad \theta_{(+3)}=\theta_{(-3)}$. Then $\widetilde{e}_{(01)}=e_{(01)}+e_{1} x_{1}, \widetilde{e}_{(02)}=e_{(02)} \cos \theta_{1}+e_{3} \sin \theta_{1}, \widetilde{e}_{(03)}=\cos \theta_{1}\left[e_{(03)}\left(1-x_{0}\right)-\bar{e}_{3} \bar{x}\right]-\sin \theta_{1}\left[e_{(2)}\left(1+x_{0}\right)+\right.$ $\left.\bar{e}_{(02)} \bar{x}\right], \widetilde{e}_{1}=e_{1}-e_{(01)} x_{1}, \widetilde{e}_{2}=e_{2} \cos \theta_{1}+e_{(03)} \sin \theta_{1}, \widetilde{e}_{3}=\cos \theta_{1}\left[e_{3}\left(1+x_{0}\right)+\bar{e}_{(03)} \bar{x}\right]-\sin \theta_{1}\left[e_{(02)}(1-\right.$ $\left.\left.x_{0}\right)-\bar{e}_{2} \bar{x}\right]$. Consequently, the resulting deformed metric on $V_{6}$ in holonomic coordinate basis takes the form $g_{00}=\tau_{1}^{2}\left(1-\tan ^{2} \theta_{1}\right)+\tau_{2}^{2} \cos 2 \theta_{1}+\tau_{3}^{2}\left\{\cos ^{2} \theta_{1}\left[\left(1-x_{0}\right)^{2}+\bar{x}^{2}\right]-\sin ^{2} \theta_{1}\left[\left(1+x_{0}\right)^{2}+\bar{x}^{2}\right]\right\}$, $g_{01}=-2 \tau_{1} x_{1}, g_{02}=2 \tau_{3} \sin 2 \theta_{1}, g_{03}=-2 \tau_{2} \sin 2 \theta_{1}, g_{11}=-\left(1+\tan ^{2} \theta_{1}\right), g_{22}=-\cos 2 \theta_{1}, g_{33}=$ $-\cos ^{2} \theta_{1}\left[\left(1+x_{0}\right)^{2}+\bar{x}^{2}\right]+$

$\sin ^{2} \theta_{1}\left[\left(1-x_{0}\right)^{2}+\bar{x}^{2}\right]$, where, the $3 \mathrm{D}$ space $T^{3}$ is spanned by the coordinates $d \widetilde{x}^{(0 \alpha)}=\tau_{\alpha} d \widetilde{x}^{0}\left(\tau_{1}^{2}+\right.$ $\left.\tau_{2}^{2}+\tau_{3}^{2}=1\right)$. At reducing $V_{6} \rightarrow V_{4}$, we may further fix the most convenient universal time direction

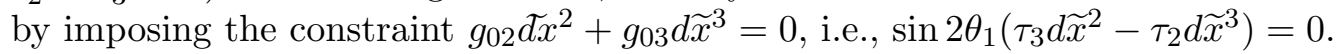

(i) In the case of static spherical-symmetry when $\sin 2 \theta_{1}=0$, the $\tau_{\alpha}$ can be chosen as $\tau_{1}=\tau_{2}=$ $0, \tau_{3}=1$.

(ii) Let, in axisymmetric space $V_{4}$, the world coordinate $\widetilde{t}\left(=\widetilde{x}^{0}\right)$ be the time (in units of c), and $\widetilde{\phi}\left(=\widetilde{x}^{1}\right)$ be the azimuthal angle about the axis of symmetry. The space $V_{4}$ would be invariant against 
simultaneous inversion of time $\widetilde{t}$ and azimuthal angle $\widetilde{\phi}$. So, $\sin 2 \theta_{1} \neq 0$, and the $\tau_{2}$ and $\tau_{3}$ can be chosen to eliminate the term $\left(\tau_{3} d \widetilde{x}^{2}-\tau_{2} d \widetilde{x}^{3}\right)$, which has the effect of introducing the following values into the problem: $\tau_{2}=\tau_{3} \frac{\widetilde{u}^{2}}{\widetilde{u}^{3}}$, at $\frac{\widetilde{u}^{2}}{\widetilde{u}^{3}} \leq 1, \quad \tau_{3}=\tau_{2} \frac{\widetilde{u}^{3}}{\widetilde{u}^{2}}$, at $\frac{\widetilde{u}^{3}}{\widetilde{u}^{2}} \leq 1$. A velocity field at each point in $V_{4}$ is $\widetilde{u}^{t}=d \widetilde{t} / d \widetilde{s}$, with proper time $d \widetilde{s}, \widetilde{t}\left(=x^{0}\right)$ is the world time coordinate (in units of c), $\widetilde{u}^{\phi}=d \widetilde{\phi} / d \widetilde{s}=\Omega \widetilde{u}^{t}$ and $\widetilde{u}^{t}=d \widetilde{x}^{C} / d \widetilde{s}=\widetilde{u}^{t} \widetilde{v}^{C}$, and $\Omega=d \widetilde{\phi} / d \widetilde{t}$ is the angular velocity, as seen by an inertial observer at rest at infinity. The components $\widetilde{v}^{C}=d \widetilde{x}^{C} / \widetilde{d t}(C=2,3)$ denote the poloidal velocity. Hence the metric on axisymmetric space $V_{4}$ becomes $g_{00}=\tau_{1}^{2}\left(1-\tan ^{2} \theta_{1}\right)+\tau_{2}^{2} \cos 2 \theta_{1}+$ $\tau_{3}^{2}\left\{\cos ^{2} \theta_{1}\left[\left(1-x_{0}\right)^{2}+\bar{x}^{2}\right]-\sin ^{2} \theta_{1}\left[\left(1+x_{0}\right)^{2}+\bar{x}^{2}\right]\right\}, g_{01}=-2 \tau_{1} x_{1}, g_{11}=-\left(1+\tan ^{2} \theta_{1}\right), \quad g_{22}=-\cos 2 \theta_{1}$, $g_{33}=-\cos ^{2} \theta_{1}\left[\left(1+x_{0}\right)^{2}+\bar{x}^{2}\right]+\sin ^{2} \theta_{1}\left[\left(1-x_{0}\right)^{2}+\bar{x}^{2}\right]$. We define the five quantities $\nu, \psi, \omega, \mu_{2}$ and $\mu_{3}$ which are only functions of the coordinate $\widetilde{x}^{2}$ and $\widetilde{x}^{3}: \exp (2 \psi)\left(\widetilde{x}^{2}, \widetilde{x}^{3}\right):=1+x_{1}^{2}, \quad \omega\left(\widetilde{x}^{2}, \widetilde{x}^{3}\right):=-\frac{\tau_{1} x_{1}}{1+x_{1}^{2}}$, $\exp (2 \nu)\left(\widetilde{x}^{2}, \widetilde{x}^{3}\right):=\frac{1}{1+x_{1}^{2}}\left\{\tau_{1}^{2}\left(1+x_{1}^{2}-x_{1}^{4}\right)+\tau_{2}^{2}\left(1-x_{1}^{2}\right)+\tau_{3}^{2}\left[\left(1-x_{0}\right)^{2}+\bar{x}^{2}\right]-\tau_{3}^{2} x_{1}^{2}\left[\left(1+x_{0}\right)^{2}+\bar{x}^{2}\right]\right\}$, $\exp \left(2 \mu_{2}\right)\left(\widetilde{x}^{2}, \widetilde{x}^{3}\right):=\frac{1-x_{1}^{2}}{1+x_{1}^{2}}, \exp \left(2 \mu_{3}\right)\left(\widetilde{x}^{2}, \widetilde{x}^{3}\right):=\frac{1}{1+x_{1}^{2}}\left\{\left(1+x_{0}\right)^{2}+\bar{x}^{2}-x_{1}^{2}\left[\left(1-x_{0}\right)^{2}+\bar{x}^{2}\right]\right\}$, where we seek a holonomic coordinates $\widetilde{x}^{\mu}(x)$, in our construction above, as the solutions of the first-order partial differential equations: $\frac{\partial \widetilde{x}^{\mu}}{\partial x^{l}} \equiv \psi_{l}^{\mu}=D_{l}^{\mu}(1+\varpi(F))$. Provided, a world-deformation tensor is in the form $\Omega_{l}^{m}(F)=\delta_{l}^{m}(1+\varpi(F))$, where $\varpi(F)$ is the scalar function of antisymmetric tensor of the gauge field. We do not at the moment specify the scalar function $\varpi(F)$ apart from the initial condition $\varpi(0)=0$, which can be determined in intermediate stage of the analysis. Substituting these solutions into a bilinear form of norm $d \widetilde{s}^{2}$ of infinitesimal displacement on $V_{4}$, describing the stationary and axisymmetric spacetimes, we obtain $d \widetilde{s}^{2}=\exp (2 \nu) d \widetilde{t}^{2}-\exp (2 \psi)(d \widetilde{\phi}-\omega d \widetilde{t})^{2}-\exp \left(2 \mu_{2}\right)\left(d \widetilde{x}^{2}\right)^{2}-$ $\exp \left(2 \mu_{3}\right)\left(d \widetilde{x}^{3}\right)^{2}$. Thus, we arrive at the metric of the stationary and axisymmetric space $V_{4}$ in the most commonly used $3+1$ formalism.

\subsection{Field equations}

In case of stationary and axisymmetric space $V_{4}$, the equations of $1 \mathrm{D}$ gravitation $x_{0}$, the framedragging potential $x_{1}$, and the ID field $\bar{x}$, generated by the stress-energy tensor of isolated spinning fluid, can be obtained in the Feynmann gauge: $\Delta x_{0}(x)=-\widetilde{J}_{\left(x_{0}\right)}(\widetilde{x}), \quad \Delta x_{1}(x)=-\widetilde{J}_{\left(x_{1}\right)}(\widetilde{x}),(\Delta-$ $\left.\lambda_{\bar{x}}^{-2}\right) \bar{x}(x)=-\widetilde{J}_{(\bar{x})}(\widetilde{x})$. Here the current $\widetilde{J}_{(\chi)}\left(\chi:=x_{0}, x_{1}, \bar{x}\right)$ is referred to as $\widetilde{J}_{(\chi)}:=(æ / 2) \sqrt{-g} e_{a}^{\mu} e_{b}^{\nu}\left(\partial g_{\mu \nu} / \partial \chi\right) \widetilde{T}^{(a b)}$, provided, the Compton length of the ID-field $\bar{x}$ is $\lambda_{\bar{x}}=\left(\hbar / m_{\bar{a}} c\right) \simeq 0.4 \mathrm{fm}$. The system of nonlinear differential field equations explicitly reads $\Delta x_{0}=-\frac{\infty}{2} \sqrt{-g}\left[\exp (-2 \nu) W^{2}\left(\widetilde{\rho}+\widetilde{P} V^{2}\right) \times \frac{\partial g_{00}}{\partial x_{0}}+\right.$ $\left.\exp \left(-2 \mu_{3}\right) \widetilde{P} \frac{\partial g_{33}}{\partial x_{0}}\right], \Delta x_{1}=-\frac{\aleph}{2} \sqrt{-g} \exp (-\nu) \frac{\partial g_{01}}{\partial x_{1}}\left[\exp (-\nu) \omega \times W^{2}\left(\widetilde{\rho}+\widetilde{P} V^{2}\right)+\exp (-\psi) W^{2}(\widetilde{\rho}+\widetilde{P}) V\right]$, $\left(\Delta-\lambda_{\bar{x}}^{-2}\right) \bar{x}=-\frac{\aleph}{2} \sqrt{-g}\left[\exp (-2 \nu) W^{2}\left(\widetilde{\rho}+\widetilde{P} V^{2}\right) \frac{\partial g_{00}}{\partial \bar{x}}+\exp \left(-2 \mu_{3}\right) \widetilde{P} \frac{\partial g_{33}}{\partial \bar{x}}\right]$. These equations can be solved together with a diffeomorphism $\widetilde{x}^{\mu}(x): M_{4} \rightarrow V_{4}$. Adopting spherical polar coordinates $(r, \theta, \phi)$ in flat space $M_{4}$, we then have $\frac{\partial \widetilde{r}}{\partial r}=\psi_{3}^{\widetilde{3}}=D_{3}^{\widetilde{3}}(1+\varpi), \quad \frac{\partial \widetilde{r}}{\partial \theta}=\psi_{2}^{\widetilde{3}}=0, \frac{\partial \widetilde{\theta}}{\partial r}=\psi_{3}^{\widetilde{2}}=0, \frac{\partial \widetilde{\theta}}{\partial \theta}=\psi_{2}^{\widetilde{2}}=D_{2}^{\widetilde{2}}(1+\varpi)$. Hence $d \widetilde{r}=\psi_{3}^{3}(\widetilde{r}, \widetilde{\theta}) d r$ and $d \widetilde{\theta}=\psi_{2}^{\widetilde{2}}(\widetilde{r}, \widetilde{\theta}) d \theta$, which means that $r=r(\widetilde{r}, \widetilde{\theta})$ and $\theta=\theta(\widetilde{r}, \widetilde{\theta})$. Therefore, the stationary axisymmetric field components $\chi(r, \theta)$ are the solutions of the system of non-linear differential axisymmetric Poisson field equations. Recall that the infinite series expansion of the factor $1 /\left|\mathbf{x}-\mathbf{x}^{\prime}\right|$ in Green function of Poisson equation in spherical polar coordinates $(r, \theta, \phi)$, in general, is $\frac{1}{\left|\mathbf{x}-\mathbf{x}^{\prime}\right|}=\sum_{l=0}^{\infty} \frac{r_{<}^{l}}{r_{>}^{l+1}} P_{l}(\cos \theta) P_{l}\left(\cos \theta^{\prime}\right)+(\phi$-dependent terms $)$, where $P_{l}$ denotes a Legendre polynomial and $r_{<}\left(r_{>}\right)$is the lesser (greater) of $r$ and $r^{\prime}$. By azimuthal symmetry, the $\phi$-dependant terms do not contribute to this relation.

\subsection{A global vacuum solution: horizons}

The existence of a global vacuum solution, $\chi\left(\equiv x_{0}, x_{1}\right)$, outside of the matter, amounts to solving the field equations in a nearly Newtonian weak source limit: $\widetilde{T} \rightarrow 0, \bar{x}=0$. In Lorentz gauge, it is given by means of fundamental solution of Poisson's equation as a retarded integral of the form familiar from linearized field equation theory: $\chi(\mathbf{x})=\frac{1}{4 \pi} \int \frac{\widetilde{J}_{(\chi)}^{\text {ext }}\left(\left|\mathbf{x}-\mathbf{x}^{\prime}\right|\right) d^{3} x^{\prime}}{\left|\mathbf{x}-\mathbf{x}^{\prime}\right|}$, where the current $\widetilde{J}_{(\chi)}^{\text {ext }}\left(\left|\mathbf{x}-\mathbf{x}^{\prime}\right|\right)$ denotes $\widetilde{J}_{(\chi)}^{\text {ext }}\left(\left|\mathbf{x}-\mathbf{x}^{\prime}\right|\right)=4 \pi Q_{(\chi)}\left(\left|\mathbf{x}-\mathbf{x}^{\prime}\right|\right) \delta\left(\left|\mathbf{x}-\mathbf{x}^{\prime}\right|\right)$. The charge $Q_{(\chi)}\left(\left|\mathbf{x}-\mathbf{x}^{\prime}\right|\right)$ is calculated in the spacetime region far outside the system, where we assume that the spacetime is almost Minkowski $g \simeq \eta$. The external metric $g^{e x t}=g^{e x t}(\chi)$ is then written $g_{00}^{e x t}=\tau_{1}^{2}\left(1-\tan ^{2} \theta_{1}\right)+\tau_{2}^{2} \cos 2 \theta_{1}+$ 

$g_{33}^{e x t}=-\cos ^{2} \theta_{1}\left(1+x_{0}\right)^{2}+\sin ^{2} \theta_{1}\left[\left(1-x_{0}\right)^{2}\right.$. These Petrov type $\mathrm{D}$ vacuum solutions associate with the gravitational field of isolated massive stationary and axisymmetric rotating SPC. They completely characterized by its mass $M_{S P C}$ and angular momentum $J_{S P C}$. The two double principal null directions define "radially" ingoing and outgoing null congruences near the SPC which is the source of the field. The horizon is a $2 \mathrm{D}$ surface of spherical topology, where the redshift factor $\alpha(r, \theta)$ vanishes $g_{00}^{e x t}(r, \theta)=\alpha^{2}(r, \theta)=\tau_{1}^{2}\left(1-\tan ^{4} \theta_{1}\right)+\tau_{2}^{2}(1-$

$\left.\tan ^{2} \theta_{1}\right)+\tau_{3}^{2}\left[\left(1-x_{0}\right)^{2}-\tan ^{2} \theta_{1}\left(1+x_{0}\right)^{2}\right]=0$. The gravitational infinite redshift suppresses any emission at the horizon. The solution, for given $x_{1}$, is $x_{0}^{( \pm)}=\left(\tau_{3}\left(1-x_{1}^{2}\right)\right)^{-1}\left\{\tau_{3}\left(1+x_{1}^{2}\right) \pm\right.$ $\left.\sqrt{\tau_{3}^{2}\left(1+x_{1}^{2}\right)^{2}-\left(1-x_{1}^{2}\right)\left(1-\tau_{2}^{2} x_{1}^{2}-\tau_{1}^{2} x_{1}^{4}\right)}\right\}$, where the discriminant has to be positive.

(i) At $x_{1}<1$, the gravitational field potentials $x_{0}^{( \pm)}$yield two physical horizons. Their radii coincide with $r_{g}=R_{g} / 2\left(x_{0}^{( \pm)}=1\right)$ on the axis of rotation of SPC, located at $\theta=0\left(\right.$ where $\left.x_{1}=0\right)$, where $R_{g}=2 G M / c^{2}=2.95 \times 10^{5} M / M_{\odot} \mathrm{cm}$ is the Schwarzschild radius (radius of a non-rotating black hole).

(ii) At $x_{1}\left(r_{0}\right)=1$, the outer oblate horizon can be formed only at infinity $r_{0} \rightarrow \infty$, where $x_{0}\left(r_{0} \rightarrow \infty\right) \rightarrow 0$.

(iii) For vanishing $x_{1} \rightarrow 0$, the solution, at $\tau_{3} \rightarrow 1\left(\tau_{2}=\tau_{1} \rightarrow 0\right)$, becomes static, spherically symmetric, yielding a single spherical event horizon $x_{0}^{( \pm)}(r) \rightarrow 1$ of previous model of non-rotating SPC.

The horizons are null surfaces because they are light-like. The hypersurfaces with $r_{ \pm}$are Killing horizons of the Killing vector field. A null hypersurface $\mathcal{N}$ is a Killing horizon of a Killing vector $\xi$ if, on $\mathcal{N}, \xi$ is normal to $\mathcal{N}$.

Suppose we adopt affine parametrization $l \cdot D l^{\mu}=0$. Since $\xi=f l$ on $\mathcal{N}$ for some function $f$, it follows that $\xi \cdot D \xi^{\mu}=k \xi^{\mu}$ on $\mathcal{N}$, where $k=\xi \cdot \partial \ln |f|$ is called the surface gravity. Since $\xi$ is normal to $\mathcal{N}$, Frobenius theorem implies that $\left.\xi_{[\mu} D_{\nu} \xi_{\rho]}\right|_{\mathcal{N}}=0$, where '[...]' indicates total anti-symmetry in the enclosed indices. For a Killing vector field $\xi$, it implies $D_{\mu} \xi_{\nu}=D_{[\mu} \xi_{\nu]}$. Except at points for which $\xi=0$, one then has $k^{2}=\left.(1 / 2)\left(D^{\mu} \xi^{\nu}\right)\left(D_{\mu} \xi_{\nu}\right)\right|_{\mathcal{N}}$. All points at which $\xi=0$ are limit points of orbits of $\xi$ for which $\xi \neq 0$, so continuity implies that this formula is valid even when $\xi=0$. One can then prove that $k$ is constant on orbits of $\xi: \xi \cdot \partial k^{2}=-\left(D^{\mu} \xi^{\nu}\right) R_{\nu \mu \rho \sigma} \xi^{\rho} \xi^{\sigma}=0$, because of antisymmetry $R_{\nu \mu \rho \sigma}=-R_{\nu \mu \sigma \rho}$.

The surface gravity $k$ is not a property of $\mathcal{N}$ alone, it also depends on the normalization of $\xi$, because if $\mathcal{N}$ is a Killing horizon of $\xi$ with surface gravity $k$, then it is also a Killing horizon of $c \xi$ with surface gravity $c^{2} k$ for any constant $c$. There is no natural normalization of $\xi$ on $\mathcal{N}$ since $\xi^{2}=0$ there, but in an asymptotically flat spacetime there is a natural normalization at spatial infinity, e.g. for the time-translation Killing vector field $k$ we choose $k^{2} \rightarrow-1$ as $r \rightarrow \infty$. This fixes $k$, and hence $k$, up to a sign, and the sign of $k$ is fixed by requiring $k$ to be future-directed.

\subsection{External fields in the weak source limit}

The external fields $\chi\left(\left|\mathbf{x}-\mathbf{x}^{\prime}\right|\right)$ can be obtained straightforwardly in the weak source limit $\widetilde{T} \rightarrow 0$, where the spacetime is almost Minkowski $g \simeq \eta$. It is sufficient then to consider an isolated system only in the asymptotic linearized regime. Without loss of accuracy, one can therefore keep only leading linear order-terms of fields, ignoring a second and higher order effects.

Linearized field equations guarantee conservation of 4-momentum and angular momentum of any body bounded by vacuum. So, we expand the solutions $\chi\left(\left|\mathbf{x}-\mathbf{x}^{\prime}\right|\right)$ in powers of $x^{\prime} / r=x^{\prime} /|\mathbf{x}|$. It is convenient to perform calculation in the system's rest frame, where $P^{j}=\int T^{j 0} d^{3} x=0$, with origin of coordinates at the system's center of mass $\int x^{j} T^{00} d^{3} x=0$. Hence, in suitable asymptotically Minkowski coordinates, where the stationary Killing vector is given by $m=\partial_{t}$, the resulting redshift factor $\alpha(M, r)$ and the frame-dragging potential $\omega(J, r)$ can be defined by means of the constants $M$ and $J$, respectively, as the total mass and intrinsic angular momentum of the source.

These quantities are well substantiated by Komar integral. Recall that to every Killing vector field $\xi$ in the volume $V$ of spacetime on a spacelike hypersurface $\Sigma$, with boundary $\partial V$, one can associate the Komar integral $Q_{\xi}(V)=(q / 16 \pi G) \oint d S_{\mu \nu} D^{\mu} \xi^{\nu}$, for some constant $q$. Since Killing fields satisfy 
the identity $D_{\nu} D_{\mu} \xi^{\nu}=R_{\mu \nu} \xi^{\nu}$, where $R_{\mu \nu}$ is the curvature tensor of $V_{4}$, then $Q_{\xi}(V)=\int_{V} d S_{\mu} J_{(\xi)}^{\mu}$, and the current $J_{(\xi)}^{\mu}$ is defined as follows: $J_{(\xi)}^{\mu}:=q R_{\nu}^{\mu} \xi^{\nu}$. The current $J_{(\xi)}^{\mu}$ is conserved $D_{\mu} J_{(\xi)}^{\mu}=0$, and the charge $Q_{\xi}(V)$ is time-independent, provided $J_{(\xi)}^{\mu}$ vanishes on the boundary $\partial V$.

If $\xi=k, q=-2$ is fixed by comparison with the formula derived for total mass (energy) in asymptotic expansion, the $M$ integral can be recast into a coordinate-independent form $M=$ $-(1 / 8 \pi G) \oint_{\infty} d S_{\alpha \beta} D^{\alpha} k^{\beta}$.If $m=\partial_{t}$ is the Killing vector of axisymmetry, one obtains for $q=1$ a coordinate-independent angular momentum integral $J=(1 / 16 \pi G) \oint_{\infty} d S_{\alpha \beta} D^{\alpha} m^{\beta}$. For a weak source, $g \simeq \eta$, the total mass and angular momentum of an asymptotically flat spacetime is found by taking $\partial V$ to be a 2 -sphere at spatial infinity. If one chooses $V$ to be on $t$ constant hypersurface, then in Cartesian coordinates $x^{i}(i=1,2,3)$, one has $d S_{\mu} m^{\mu}=0$ and $m=x^{1} \partial_{2}-x^{2} \partial_{1}$. For a weak source, therefore $J(V) \simeq \varepsilon_{3 i j} \int_{V} x^{i} T^{j 0}$.

Using Killing equations $D_{(\alpha} k_{\beta)}=0=D_{(\alpha} m_{\beta)}$, which lead to $D_{\alpha} D^{\alpha} k^{\beta}=R_{\rho}^{\beta} k^{\rho}$ and $D_{\alpha} D^{\alpha} m^{\beta}=$ $R_{\rho}^{\beta} m^{\rho}$, the total mass and angular momentum above can be converted by means of Stoke's theorem as $M=-(1 / 4 \pi G) \int_{\Sigma} R_{\beta}^{\alpha} k^{\beta} d \Sigma_{\alpha}, J=(1 / 8 \pi G) \int_{\Sigma} R_{\beta}^{\alpha} m^{\beta} d \Sigma_{\alpha}$. The leading linear order-terms in asymptotic expansion of the redshift factor and the frame-dragging potential are given by $\alpha\left(R_{g}, r\right) \simeq$ $1-R_{g} / 2 r+O\left(1 / r^{3}\right), \omega(J, r)=2 G J / c^{3} r^{3}+O\left(1 / r^{4}\right)$. Equating these values respectively to the functions $\left[g_{00}^{e x t}(M, r)\right]^{1 / 2}$ and $\left[-(1 / 2) g_{01}^{e x t}(J, r)\right]$, where the components of the external metric, accurate to linearized order, become $g_{00}^{\text {ext }} \simeq 1-2 \tau_{3}^{2} x_{0}$, and $g_{01}^{\text {ext }}=-2 \tau_{1} x_{1}$, we finally obtain $x_{0}(r)=$ $R_{g} / 2 \tau_{3}^{2} r, \quad x_{1}(r)=2 G J_{S P C} / \tau_{1} c^{3} r^{3}$. The potential of dragging of inertial frames $\omega$ drops very rapidly with increasing radius. The maximal frame-dragging effects therefore can only be observed in the immediate vicinity of the event horizon: $x_{1(H)}=\omega_{H} / \tau_{1} \simeq 2 G J_{S P C} / \tau_{1} c^{3} R_{g}^{3}=J_{S P C} c^{3} / 4 \tau_{1} G^{2} M^{2}$. In physical units the angular momentum $J_{S P C}=a M_{S P C}$ becomes $J_{S P C}=a_{*} M_{S P C} \frac{G M_{S P C}}{c^{2}}$, such that $x_{1(H)}=\left(2 c a_{*} / \tau_{1} G M_{S P C}\right)$, where $a_{*}$ is the dimensionless angular momentum $\left|a_{*}\right| \leq 1$. The special case $a=M_{S P C}$ is the extreme solution. From this we get the maximal specific angular momentum $J_{S P C}^{\max }=\left(G M_{S P C} / c\right)$, and that $x_{1(H)}=2 a_{*} / \tau_{1} J_{S P C}^{\max }<1$.

The Petrov type D vacuum solutions for stationary axisymmetric rotating SPC, therefore, satisfy the Robinson's theorem for Kerr solutions in vacuum: the solutions, (i)-are asymptotically flat, (ii)contain a smooth convex horizon, (iii)- are nonsingular outside the horizon, and are uniquely specified by two parameters: the mass $M_{S P C}$ and angular momentum $J_{S P C}$. The angular velocity of a SPC is the sum of two terms: the classical one given by the intrinsic angular velocity $\Omega$ and the frame dragging $\omega$ from the rotation of absolute space.

Near the horizon of SPC, for example, where the redshift tends to zero $(\alpha \rightarrow 0)$, the angular velocity of matter $\Omega$ is completely dominated by the frame-dragging effect. Whatever the intrinsic angular momentum of the incoming matter is, this matter is forced to rotate with the local angular velocity $\omega$, which is the maximal angular velocity at event horizon. When matter falls, say into a nonrotating black hole, it is forced to zero rotation near the horizon despite its angular momentum.

The event horizon is a Killing horizon for the Killing field $\xi=k+\Omega_{H} m$, with $\xi^{2}=0$, where $\Omega_{H}$ denotes the angular velocity of the horizon as it is rigidly rotating. Let $m=\partial_{t}$ be the axial Killing field, and $\widetilde{u}=\widetilde{u}^{t}\left(1, \Omega_{H}, 0,0\right)$ be the four-velocity of SPC. We may choose $\tau_{2}=0, \tau_{1}=\tau_{3}=1 / \sqrt{2}$. Since $x_{1(H)}<1$, analogous to the Kerr black hole, there are the outer horizon or event horizon by its radius $r_{+}$, and the inner horizon or Cauchy horizon by its radius $r_{-}$. The radii $r_{+}$and $r_{-}$coincide with $r_{g}\left(x_{0}^{( \pm)}=1\right)$ on the axis of rotation of SPC, located at $\theta=0\left(J_{S P C}=0\right)$. The oblate event horizon is the surface of the oblate spheroid with the semimajor $r_{+}$and semiminor $r_{g}$ axes, respectively. Dependent of the value of the potential $x_{1(H)}$, the Cauchy horizon either is the surface of the prolate spheroid with the semimajor $r_{g}$ and semiminor $r_{-}$axes, or oblate spheroid with the semimajor $r_{-}$ and semiminor $r_{g}$ axes, respectively. The radii of the oblate event horizon and the Cauchy horizon are given by $r_{\mp}=r_{g} / \tau_{3}^{2} x_{0}^{( \pm)}$. These radii, as the function of $x_{1(H)}$, are plotted in Fig. 10 . Since $\alpha\left(r_{+}\right)=0$, any observer, but the ZAMO, rotates at the outer horizon with the angular velocity $\Omega_{H}$. The angular velocity of ZAMO is $\Omega=\omega$, which will have vanishing specific angular momentum: $\widetilde{u}^{\phi}=g_{\phi \alpha} \widetilde{u}^{\alpha}=g_{\phi \phi} \widetilde{u}^{\phi}+g_{t \phi} \widetilde{u}^{t}=\widetilde{u}^{t}\left(\omega g_{\phi \phi}+g_{t \phi}\right)=\widetilde{u}^{t} g_{\phi \phi}\left(\omega+g_{t \phi} / g_{\phi \phi}\right)=0$. It is therefore convenient to express physical observables with respect to ZAMO. In the slow-rotation limit, the angular momentum of SPC, as rigidly rotating body, can be given in the form $J=\int_{M_{H}}(\widetilde{\rho}+\widetilde{P})\left(\widetilde{u}^{t}\right)^{2}\left(g_{t \phi}+\Omega g_{\phi \phi}\right) \sqrt{-g} d^{3} \widetilde{x} \simeq$ 


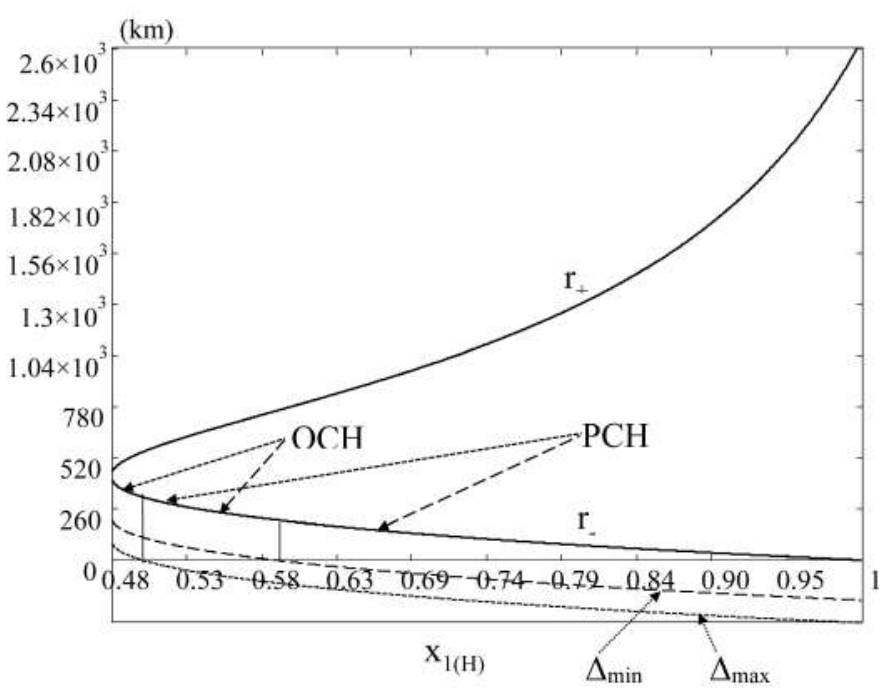

Figure 10. The radii $r_{ \pm}$of outer and inner horizons v.s. potential $x_{1(H)}$, for M82X-2, with the mass $M \simeq 138.5-226 M_{\odot}$, i.e. $r_{g}=R_{g} / 2=r_{g}^{\max }-r_{g}^{\min } \simeq 204.3-333.4 \mathrm{~km}$.

Abbreviated notations: $\Delta_{\min }:=r_{-}-r_{g}^{\min }, \Delta_{\max }:=r_{-}-r_{g}^{\max }, \mathrm{OCH}:=$ Oblate Cauchy Horizon, PCH $:=$ Prolate Cauchy Horizon.

$\int_{M_{H}}(\widetilde{\rho}+\widetilde{P})\left(\widetilde{u}^{t}\right)^{2} g_{\phi \phi}\left(\Omega_{H}-x_{1(H)} / \sqrt{2}\right) \sqrt{-g} d^{3} \widetilde{x}$, which in leading order in the expansion for $\Omega_{H}$ reads $J \simeq \frac{8 \pi}{3} \int_{0}^{R_{S P C}} r^{4} \frac{(\rho+P)}{\left(1-r_{g} / r\right) \sqrt{1-2 M(r) / r}}\left(\Omega_{H}-2 a_{*} / 4 J_{S P C}^{\max }\right) d r$, where $R_{S P C}$ is the radius of SPC.

\subsection{Microscopic model of stationary and axisymmetric rotating SPC}

The key physical properties of SPC depend very little on the details of concrete SPC-model, as they are a direct consequence of the fundamental features of underlying gravitation theory. The latter explores a spontaneous breaking of gravitation gauge symmetry and a rearrangement of vacuum state. We therefore expect that the key physical properties of non-rotating SPC, outlined in Section 9, even though without being carefully treated, retain for a rotating SPC too. Some evidence for a simplified physical picture, for example, at $x_{1}<1$ in the weak source limit (iii), is highlighted in Fig. 11 to guide the eye, without loss of generality. Model building of the periodic source M82X-2. With this guidelines to follow, we may proceed to Fig. 12 and Fig. 13. Fig. 12 emphasizes an apparent distinction between Kerr model and rotating SPC in second half of its lifetime. Fig. 13 schematically plot the SIMBH model of the periodic ULX M82X-2, constituting mass-exchange binary with the $\mathrm{O} / \mathrm{B}$-type donor star. The OEH-surface of the spheroid has the polar equation $R=R(\vartheta)$, where $\frac{r_{g}^{2}}{R^{2}}=\cos ^{2} \vartheta+\left(1-e^{2}\right) \sin ^{2} \vartheta$, provided, $\vartheta$ is the reduced or parametric latitude $(-\pi / 2<\vartheta<\pi / 2), e$ denotes the eccentricity $e:=e\left(x_{1(H)}\right)=\sqrt{1-r_{g}^{2} / r_{+}^{2}\left(x_{1(H)}\right)}$, while $r_{+}\left(x_{1(H)}\right)$ and $r_{g}:=R(0)$ are the semimajor and semiminor axes, respectively, of the rotated ellipse of $\mathrm{OEH}$.

\subsection{Corrections to the characteristic phase profile of M82 X-2 introduced by OEH}

The OEH introduces the corrections to the characteristic phase profile of previous model of X-ray radiation from M82X-2 derived in approximation of spherical EH. In the case of OEH, the pulsed luminosity reads (Ter-Kazarian \& Shidhani, 2017): $\widetilde{L}\left(M, d, T_{s}, e, \theta, \phi, \alpha, t\right)=S_{z x} \sigma T_{s}^{4}=L_{0}\left(M, d, T_{s}\right) \Phi^{\prime}(e, \theta, \phi, \alpha, t)$, where the amplitude is $L_{0}\left(M, d, T_{s}\right) \simeq 1.05 \times 10^{4}\left(\mathrm{erg} \mathrm{s}^{-1}\right) \frac{M}{M_{\odot}} \frac{d}{\mathrm{~m}} \frac{T_{s}^{4}}{K^{4}}$, and the corrected phase profile $\Phi^{\prime}(e, \theta, \phi, \alpha, t)$ is $\Phi^{\prime}(e, \theta, \phi, \alpha, t)=\Upsilon(e, \alpha) \Phi(\theta, \phi, \alpha, t)$. Provided, the correction function is denotetd by $\Upsilon(e, \alpha):=C_{1}(e, \alpha) / 2 \pi r_{g}, \Phi(\theta, \phi, \alpha, t)$ is the phase profile in case of spherical EH: $\Phi(\theta, \phi, \alpha, t) \equiv$ $\sqrt{1-\sin ^{2} \beta \sin ^{2}(\phi+A)}$. Here the spherical triangle, with the lengths of three sides $\theta=\widehat{(z, s)}, \alpha=$ $\widehat{(s, n)}$ and $\beta=\widehat{(z, n)}$, is solved by the law of $\operatorname{cosines} \cos \beta(\theta, \alpha, t)=\cos \theta \cos \alpha+\sin \theta \sin \alpha \cos \Omega t$, $\cos A(\theta, \alpha, t)=\frac{\cos \alpha-\cos \theta \cos \beta}{\sin \theta \sin \beta}$. Consequently, the phase profile can be recast into the form $\Phi^{\prime}(e, \theta, \phi, \alpha, t)$ 

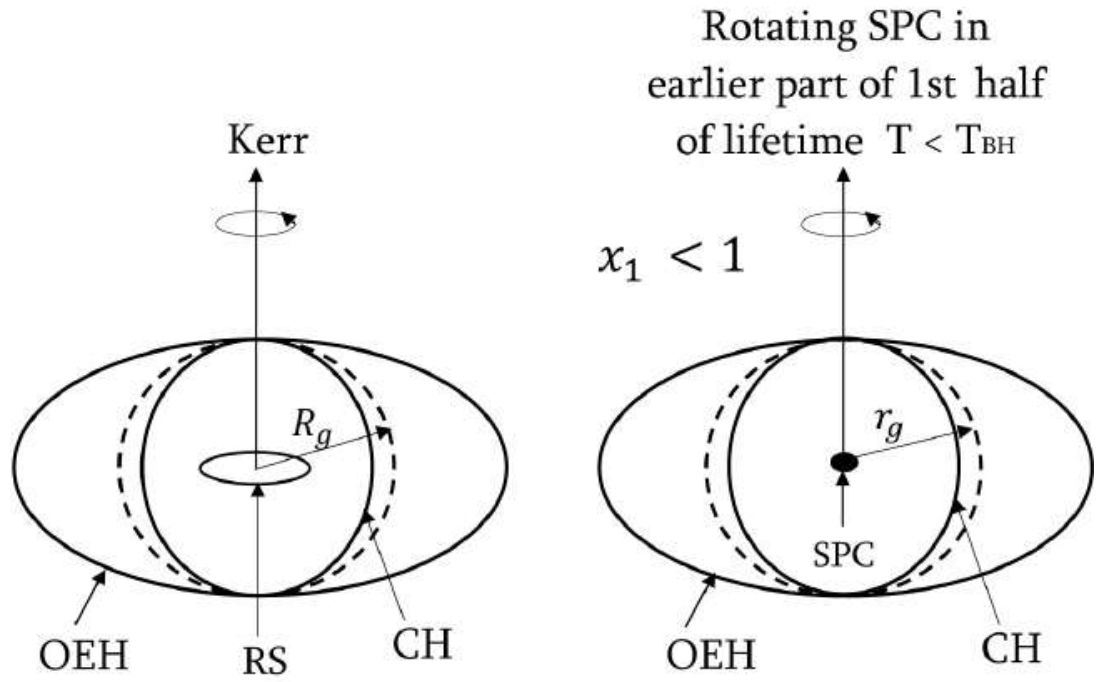

Figure 11. Left panel: Kerr model of spinning black hole. The meaningless ring singularity occurs at the center inside the black hole. Right panel: Microscopic model of rotating SPC in earlier part of first half of its lifetime $T<T_{B H}$. The picture is not to scale.

Abbreviated notations: OEH $:=$ Oblate Event Horizon, SPC $:=$ Superdense Proto-matter Core, RS :=Ring Singularity, PCH := Prolate Cauchy Horizon.

$=\frac{1}{\sqrt{1-e^{2}}}\left[1-\sum_{n=1}^{\infty}\left(\frac{(2 n-1) ! !}{2^{n} n !}\right)^{2} \frac{e_{1}^{2 n}(\alpha)}{2 n-1}\right] \Phi(\theta, \phi, \alpha, t)$, where $n ! !$ is the double factorial $n ! !=\prod_{i=0}^{l}(n-2 i)$, $l=[n / 2]-1$. The Fig. 14 - Fig. 18 are the examples, revealing the diversity of the behavior of corrected phase profiles versus the time, viewed at given position angles, for different values of eccentricity.

\section{A study of intermediate mass black hole-candidates}

A good deal of evidence that IMBHs could be the seeds for the growth of SMBHs. The IMBHs are the long-sought missing population falling within the gap between stellar mass BHs, $\sim 3-100 M_{\odot}$, and SMBHs. The leftover populations of IMBHs at low redshift, i.e. those seed BHs that did not grow into SMBHs (the leftovers of the early Universe), are expected to be found in the local Universe and up to $z \sim 2.4$, namely in dwarf star-forming galaxies, as these have undergone a quieter merging/accretion history; in nearby globular clusters; or in the form of off-nuclear ultraluminous and hyperluminous X-ray sources (ULXs and HLXs) in the halos and spiral arms of large galaxies. We need hardly add that the study of an accretion physics of IMBHs, incorporating their observed number so far available on over the last decades, has been a prompt for pushing forward many intriguing proposal towards formation and growth of initial seed BHs. These scenarios view IMBHs as a laboratory to giving hints as to the proper path toward the formation of the first galaxies and the quenching of star formation, for studies of the epoch of reionization, and for proving, in general, that a disk-jet coupling mechanism takes places in BHs of all masses. There are three formation scenarios for IMBHs: (i) the merging of stellar mass BHs and other compact objects; (ii) the runaway collision of massive stars in dense stellar clusters and the collapse of the collision product into an IMBH; (iii) they are primordial BHs formed in the early Universe being extremely dense. In such a dense medium, there must have been initial density perturbations that could then grow under their own gravity. Different models for the early universe vary widely in their predictions of the scale of these fluctuations.

Without care of the historical justice and authenticity, it should be emphasized that the ultraluminous X-ray sources (ULXs) have attracted a great deal of observational and theoretical attention, in part because their luminosities suggest that they may harbor IMBHs with an ubiquitous feature of the mass fits of more than $10^{2}-10^{4} M_{\odot}$. A strong argument in favor of IMBHs is the presence of a soft, $0.1-0.2 \mathrm{keV}$ component in their spectra. In addition to the search for HLXs, IMBHs can, in principle, be discovered in the center of low-luminosity active galactic nuclei (LLAGN), and dwarf 
Rotating SPC in

2nd half of lifetime

$\mathrm{T} \geq \mathrm{T}_{\mathrm{BH}}$

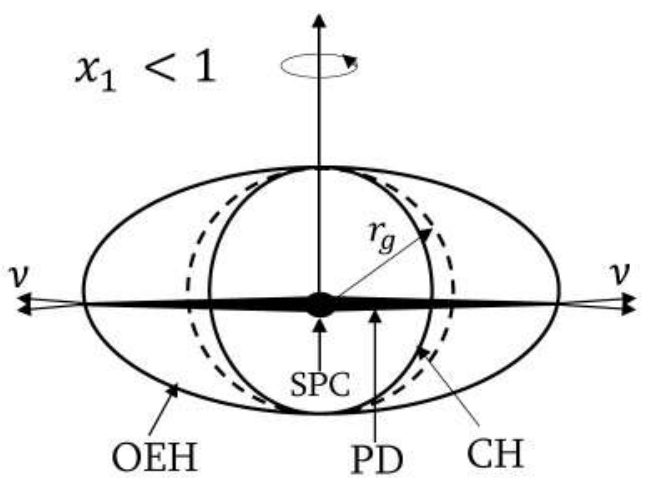

Figure 12. Microscopic model of rotating SPC in second half of its lifetime. An infalling matter already formed a thin co-spinning proto-matter disk which has reached out the edge of the outer oblate event horizon. A singularity inevitably disappears and the neutrinos escape to outside world through the vista.

Abbreviated notations: OEH $:=$ Oblate Event Horizon, SPC $:=$ Superdense Proto-matter Core, PCH $:=$ Prolate Cauchy Horizon, PD $:=$ Proto-matter Disk.

galaxies. These low-mass galaxies are expected to have undergone quiet merger histories and are, therefore, more likely to host lower-mass central BHs, a fraction of which is expected to lie in the IMBH range. However, it is premature, therefore, to draw conclusions and only time will tell whether any of these intriguing proposals is correct and which of the hypothesized IMBH formation scenario is actually realized in nature.

The MTBH allows to construct also microscopic models of 137 plausible accreting IMBH-candidates (Ter-Kazarian \& Shidhani, 2019). In the sequel we have established that versus a broad range of the central values of physical parameters, such configurations present the microscopic models of IMBHs. The mass estimates collected from the literature of all the observational evidence for 137 IMBHcandidates, even thought there are still large uncertainties, allow us to undertake a large series of numerical simulations to obtain all their physical characteristics. Below we briefly reflect upon a few key points.

\subsection{Primer on the microscopic models of IMBHs}

In our setting we retain the rather concrete proposal of preceding developments of the model of a non-rotating SPC described in Section 8, without going into the subtleties, as applied to the study of IMBHs. For brevity reasons, we proceed in relatively simple way toward first look at some of the associated physics, which is quick to estimate the physical properties of more realistic SPCconfigurations. The equations describing the equilibrium SPC include the gravitational and ID field equations, the hydrostatic equilibrium equation, and the state equation of the spherical-symmetric distribution of baryonic-quark matter in many-phase stratified states specified for each domain. The last equation will be discussed separately in the next Section step-by-step away from the domain of lower density up to the domain of higher density. We implement our scheme by considering the equilibrium configurations of non-rotating SPC of the two classes as described in secttion 8, with spherical-symmetric distribution of matter in many-phase stratified states. A layering of configuration is a consequence of the onset of different regimes in equation of state. 


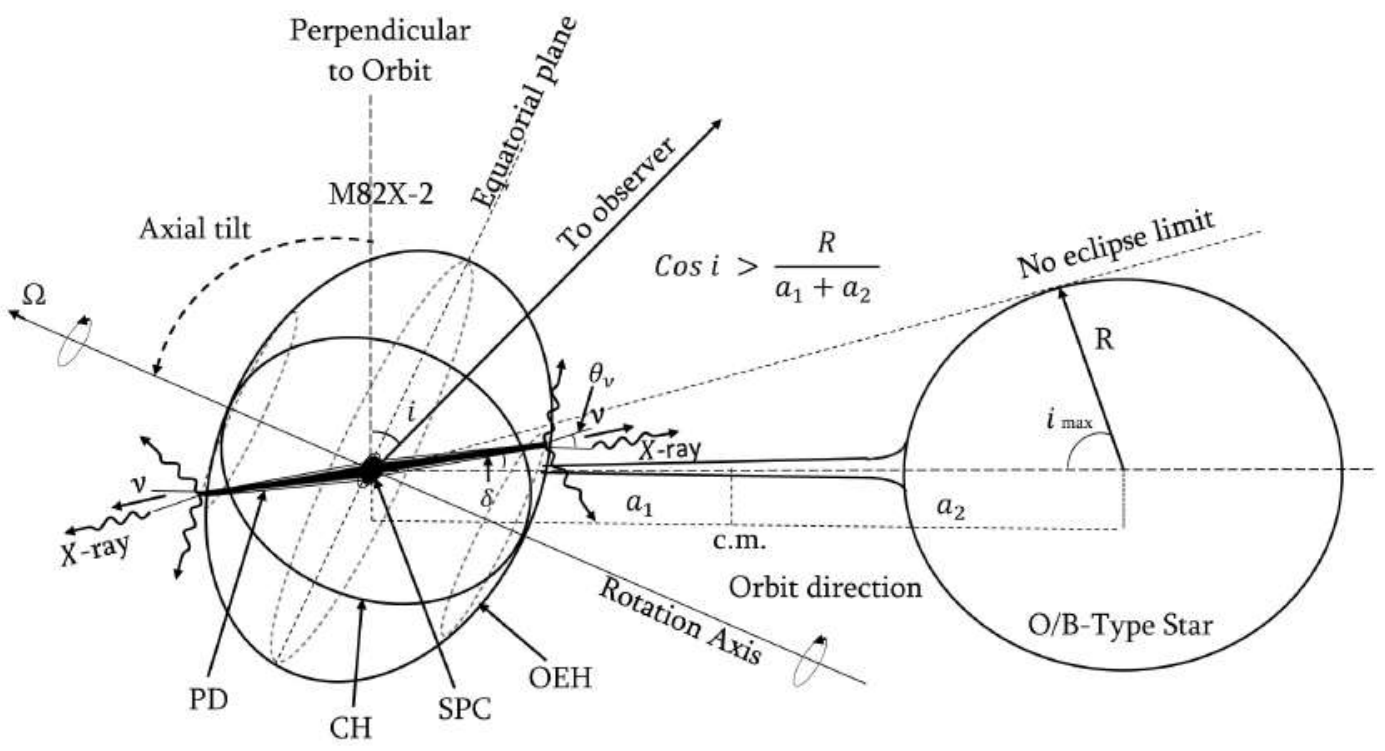

Figure 13. A schematic SIMBH model of M82X-2 constituting mass-exchange binary with the O/Btype donor star. The angle $i$ is the binary inclination with respect to the plane of the sky. No eclipse condition holds. In final stage of growth, PD has reached out the edge of the OEH. The thermal defuse blackbody X-rays beams may escape from SIMBH through a thin belt area $S=2 \pi R(\theta) d$ to outside world that sweep past Earth like lighthouse beacons. Parameters of a binary system is viewed in the orbital plane. The picture is not to scale.

Abbreviated notations: OEH $:=$ Oblate Event Horizon, SPC $:=$ Superdense Proto-matter Core, PCH $:=$ Prolate Cauchy Horizon, PD $:=$ Proto-matter Disk.

\subsection{Simulations and the results}

We are led to the numerical integration of equations of equilibrium SPC-configurations, including the state equation specified for each domain at ID-regime, leading from the center of configuration up to the surface. For the central parameters given in table 1 of (Ter-Kazarian \& Shidhani, 2019), the results of the numerical integration of the equations of I-class configurations of 137 IMBHs are presented in table 2 of (Ter-Kazarian \& Shidhani, 2019). Similar reasonings lead us also to compute corresponding characteristics referring to the II-class configurations. But it is not convenient to bring here the table data, as they are too large. Moreover, they are rather lame to specify completely all the relations between the integral characteristics. Therefore, the most straightforward and perhaps natural way to proceed is to present the figures, which clearly highlight a main difference between both classes. For 137 IMBHs of both class configurations, the masses $M, M_{1}$ and $M_{0}$, the number of baryons and the gravitational packing coefficients are given in Fig.17 versus central density and radius. The radial profiles of the pressure and the density; the gravitational $\left(x_{0}\right)$ - and ID $(x)$ - potentials; the $g_{00}$ and $g_{33}$ metric components; the ratios of electron-neutron (en), electron-proton (ep) and protonneutron (pn) concentrations are plotted in Fig.18 for the sources M82X-1. The state equations for the sources M82 X-1 and IC 467 are presented in Fig.19. As in Section 10, the special units in use, denote $P_{O V}=6.469 \times 10^{36} \mathrm{erg} \mathrm{cm}^{-3}, \quad \rho_{O V}=7.195 \times 10^{15} \mathrm{~g} \mathrm{~cm}^{-3}$ and $r_{O V}=13.68 \mathrm{~km}$.

Cutting short where our analysis is leading to, we note that there is still very much to be gained by further study of the issues that we raised here. 

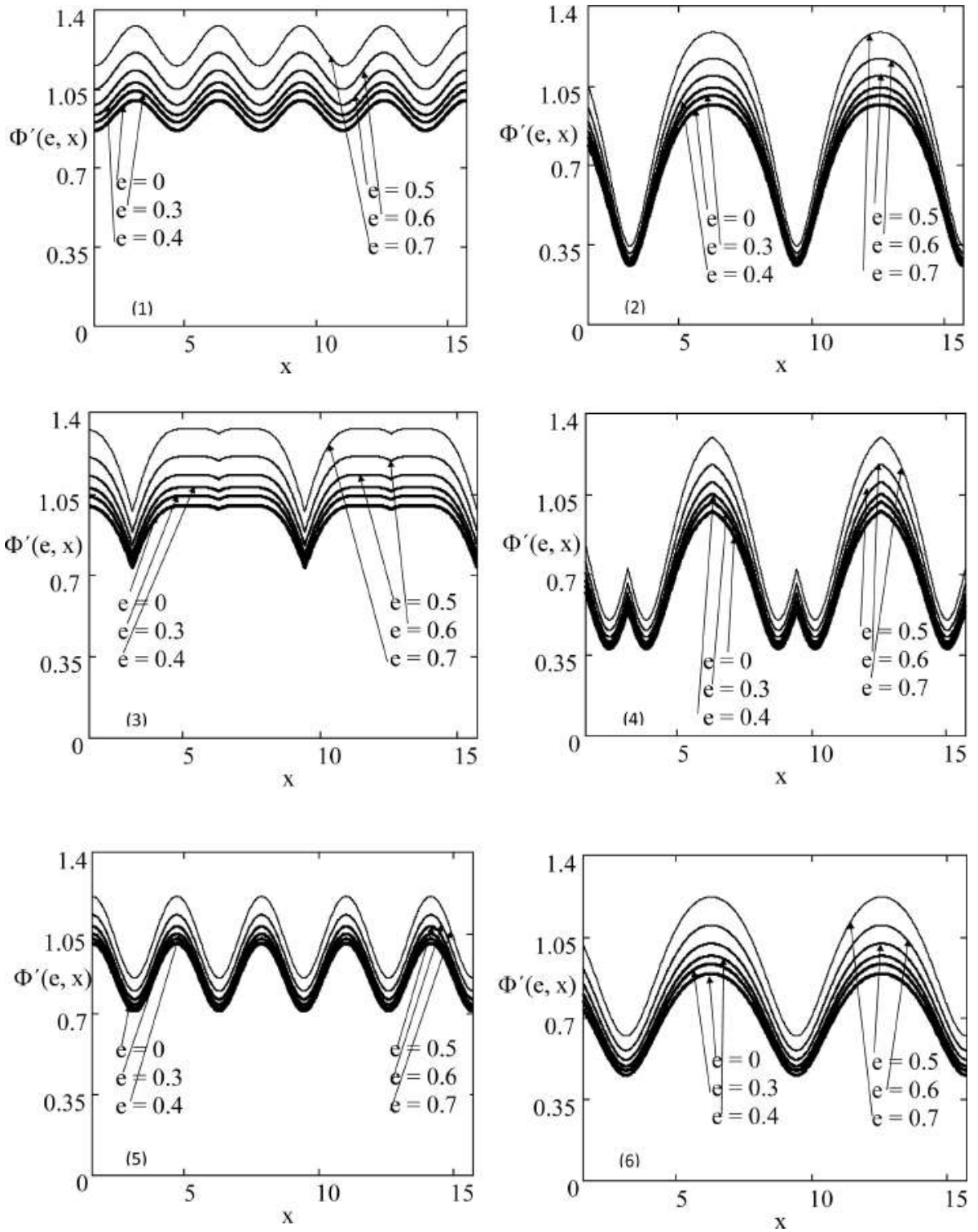

Figure 14. Corrected characteristic phase profiles versus the time $(x \equiv \Omega t)$, viewed at the following position angles $(\theta, \alpha, \phi)$ : (1) $\left(45^{0}, 30^{0}, 0^{0}\right) ;(2)\left(45^{0}, 30^{0}, 90^{0}\right) ;(3)\left(45^{0}, 30^{0}, 135^{0}\right) ;(4)\left(45^{0}, 30^{0}, 60^{0}\right)$; (5) $\left(45^{0}, 90^{0}, 90^{0}\right) ;(6)\left(45^{0}, 18^{0}, 90^{0}\right)$.

\section{The physical outlook and concluding remarks}

In this Section we briefly expose the main features of our physical outlook and draw a number of conclusions. The following items once again resume a whole physical picture.

- Ambartsumian's stemming idea of stellar association as a dynamical entity of groups of stars with positive total energy had far-reaching implications for subsequent star formation theories. Based on the observations in 1947 of stars of $\mathrm{O}$ and $\mathrm{B}$ spectral types and $\mathrm{T}$ Tauri and flare stars that cluster very loosely, Ambartsumian suggest a concept of $\mathrm{OB}$ and $\mathrm{T}$ associations, coined by him as Stellar Associations. These considerations prove that the ideas about the simultaneous emergence of stars and diffuse matter from denser and more massive bodies are more fruitful and correspond to reality. In these associations the stars differ from the surrounding stars by having a greater partial density.

In 1953, Ambartsumian paid attention to the fact that in short burst periods, UV Ceti type variable stars acquire some features similar to characteristics of $\mathrm{T}$ Tauri stars, and that the T Tauri type stars along with continuous and irregular changes of brightness also show flare type changes. He con- 

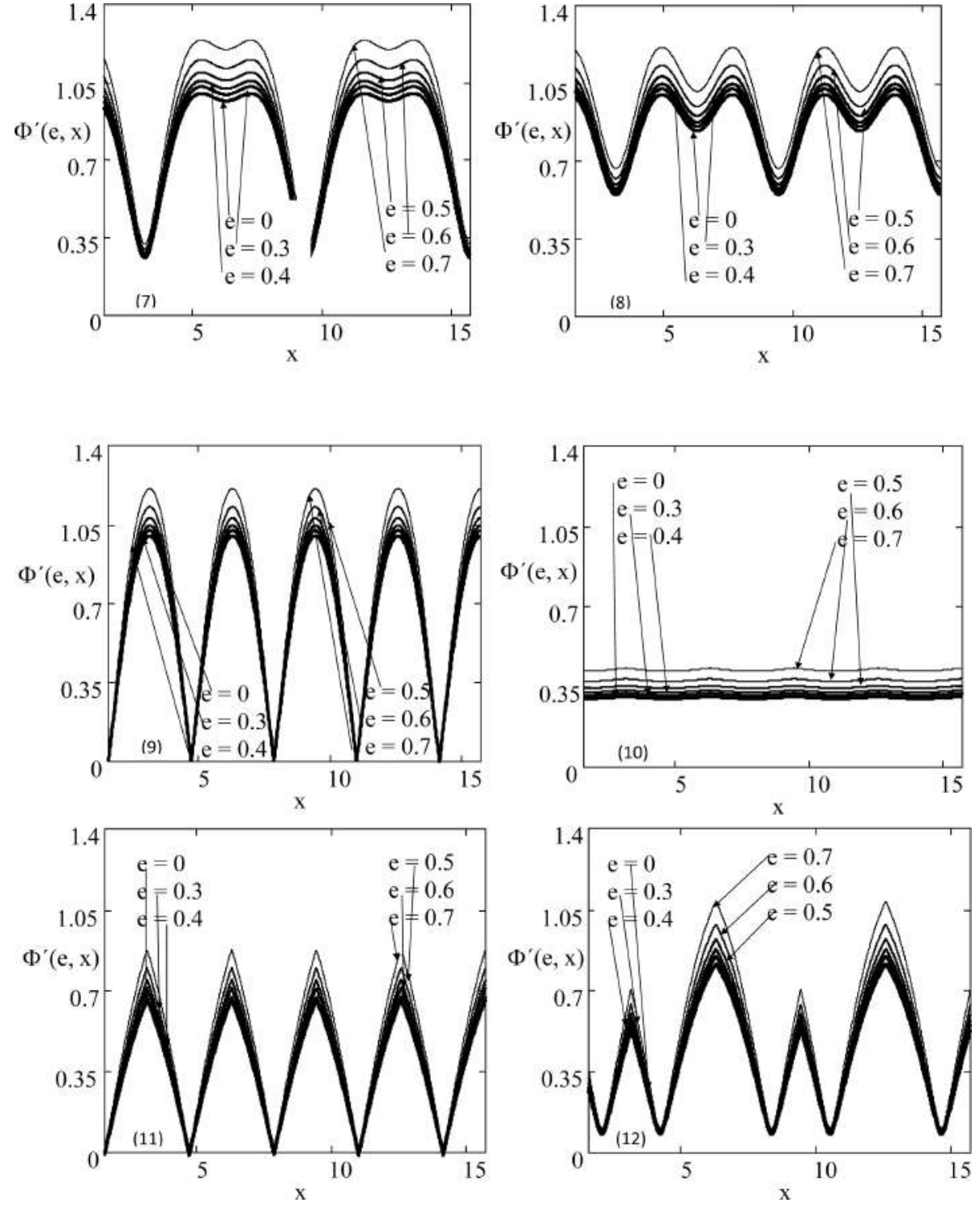

Figure 15. Corrected characteristic phase profiles versus the time $(x \equiv \Omega t)$, viewed at the following position angles $(\theta, \alpha, \phi)$ : (7) $\left(45^{0}, 60^{0}, 90^{0}\right)$; (8) $\left(45^{0}, 78.3^{0}, 90^{0}\right)$; (9) $\left(90^{0}, 90^{0}, 180^{\circ}\right)$; (10) $\left(90^{0}, 0.6^{0}, 72^{0}\right) ;(11)\left(90^{0}, 30^{0}, 60^{0}\right)$; (12) $\left(72^{0}, 30^{0}, 60^{0}\right)$.

cluded that these two types of objects are genetically related. The ultraviolet excess observed in some $\mathrm{T}$ Tauri stars has approximately the same energy distribution as the emission from flares of UV Ceti type stars. A comprehensive study of this phenomenon allows Ambartsumian in 1968 to conjecture that the flare stars are the earliest evolutionary stages of the dwarf stars. Moreover, he believes that the stage of a flare star follows a stage of $\mathrm{T}$ Tauri type at overlapping of these stages.

- Ambartsumian argued that a superdense companion in the close neighborhood of that star (red dwarf) gives the thermal radiation of the prefuor. While it cannot be ruled out that the envelope is ejected by a super-dense companion. Proceeding from the fact that prefuors and flare stars are members of the same stellar associations, Ambartsumian concluded that the processes of decay and release of energy in both cases have the same physical nature. Ambartsumian believes that each outburst is the result of the explosion (when brightness increase time is often measured in seconds) of a certain portion of the prestellar superdense matter, which came out from the inner layers of the star, where a prestellar matter remained for a relatively long time before explosion.

Ambartsumian, therefore, rejected the assumption of thermonuclear reactions as the main sources 

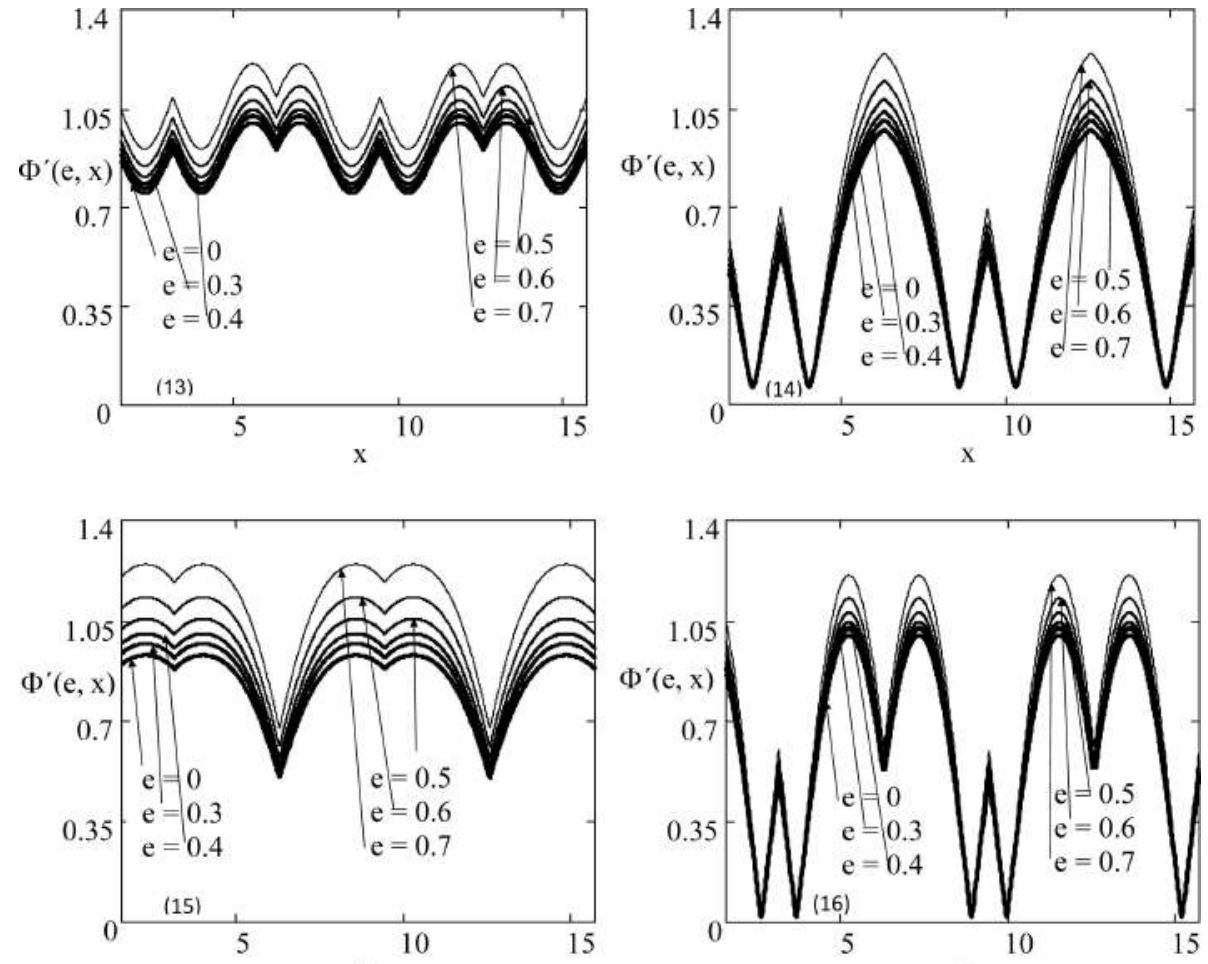

Figure 16. Corrected characteristic phase profiles versus the time $(x \equiv \Omega t)$, viewed at the following position angles $(\theta, \alpha, \phi)$ : (13) $\left(60^{0}, 90^{0}, 60^{0}\right)$; (14) $\left(60^{0}, 45^{0}, 60^{0}\right)$; (15) $\left(60^{0}, 153^{0}, 60^{0}\right)$; (16) $\left(1.2^{0}, 90^{0}, 60^{0}\right)$.

of this energy. Based on the idea that a portion of superdense prestellar matter transfers from the stellar interiors to the surface layers of young stars, Ambartsumian predicted the existence of fast and slow flares, which were later discovered, and gave an explanation to the Fuor phenomenon.

Ambartsumian predicted the expansion of the associations, which was later observed. These ideas have a subsequent influence in his interpretation of active galaxies.

Ambartsumian was also first to call attention to the instability and activity of galactic cores and, thus, raised the issue of Activity of Nuclei of Galaxies. In his report at the Solvay Conference on Physics (Brussels 1958), Armbartsumian claimed that the activity of the galactic nuclei determines the most significant processes in the evolution of galaxies. He argued that so-called D-bodies exist in nature, which are responsible for the activity of the nuclei of galaxies, and that galaxies are born from the explosion of such $D$-bodies. The nuclei of galaxies are the places where new objects are generated, and the fact that the activity can be found in galaxies with a wide range of distances strongly suggests that the activity goes on at all epochs. Violent events in galaxies are manifestations of mass creation. From these events huge fluxes of relativistic particles and rapidly moving gas clouds are generated. Quasars with intrinsic redshifts are also ejected, and they are probably related to the mechanism through which new galaxies are formed. Following Armbartsumian, it is reasonable to consider the possibility that systems of galaxies with positive total energy also originate in this way.

Ambartsumian concluded that the phenomena all pointed to ejection from superdense state, and that the galactic nuclei must contain superdense bodies of huge mass and some kind of non-stellar objects of unknown nature. He, in fact, conjectured that such an eruptive activity is due to the violent outburst events of transformations of superdense matter in supermassive compact D-bodies in galaxies, away from the accretion physics, where the creation process is at work. This signifies that every nucleus is made up of three components: stellar population, gas and supermassive body. Dynamically, the nucleus evolves independently of the rest of the galaxy. Thus, his conclusion was 
137- IMBHs (I-Class Configurations)

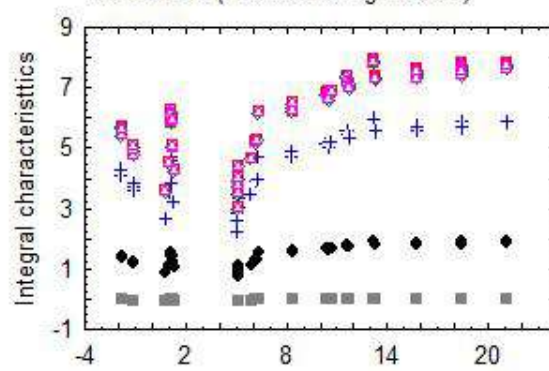

(a) $\log \left(\rho_{c} / \rho_{0}\right)$

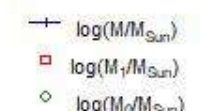

$\circ \log \left(\mathrm{M}_{0} / \mathrm{M}_{3}\right)$
137- IMBHs (II-Class Configurations)

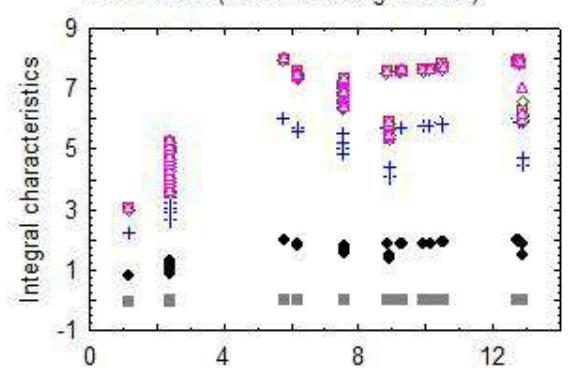

(b) $\log \left(\rho_{c} / \rho_{0}\right)$

$=\log \left(\mathrm{A}_{2}\right)$

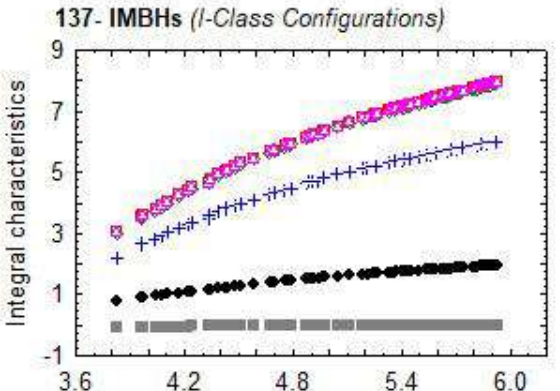

(c) $\log \mathrm{R}(\mathrm{km})$
137- IMBHs (II-Class Configurations)

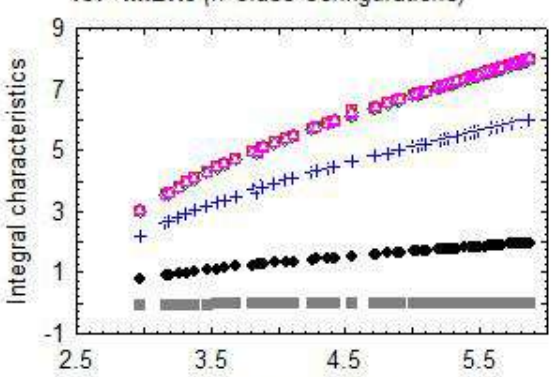

(d) $\log R(\mathrm{~km})$

Figure 17. The 137 IMBH-configurations: the masses (over solar mass), number of baryons (over $N_{0}=10^{57}$ ) and gravitational packing coefficients versus central density and radius.

that observations produce almost innumerable evidence in favor of ejections and explosions and are rather scanty regarding the processes of condensation and collapse. In the observable Universe the processes of expansion and diffusion are responsible for the majority of changes occur now. These violent explosions were the manifestations of mass creation events.

A thousands of galaxies with strong ultraviolet excess have been discovered ever since in Byurakan Observatory under his direction. Ambartsumian's student, B. Markarian (well known for Markarian galaxies), completed a brilliant survey of galaxies known by his name with UV excess using the 1-m Schmidt telescope of the Byurakan Observatory.

- The most important property of superdense states, as shown by Ambartsumian and Saakyan, must be the presence of hyperons in the star, in addition to neutrons. The temperature is assumed so low that all types of fermions are degenerate. With increasing density, different hyperons are successively appear and increase in number. They should be stable because of Pauli's exclusion principle. Paradoxically, the smallest threshold density does not correspond to the $\Lambda$-hyperon, having the smallest mass at rest, but to the $\Sigma^{-}$-hyperon. This suggests that a sufficiently massive celestial body in gravitational equilibrium should consist of a hyperon core, a neutron layer, and an outer envelope with the usual composition made of electrons, protons and composite nuclei. At density $\rho<\rho_{n}=1.28 \times 10^{7} \mathrm{gcm}^{-3}$, the gas consists of protons and electrons. At $\rho=\rho_{n}$ neutrons appear. At density $\rho>10^{8} \mathrm{gcm}^{-3}$, the number of neutron is much larger that numbers of protons and electrons. The individual atomic nuclei will no longer play an important role. At baryon density $N \geq 6.4 \times 10^{38} \mathrm{~cm}^{-3}$, i.e. $\rho=\rho_{\Sigma^{-}}=1.1 \times 10^{15} \mathrm{gcm}^{-3}$, the first hyperons appear. Although the rest masses of the hyperons $\Lambda, \Sigma^{+}, \Sigma^{0}$ are smaller than that of the $\Sigma^{-}$, nevertheless first $\Sigma^{-}$-hyperons appear. Highly degenerate matter contains hyperons and $\mu^{-}$-mesons in addition to nucleons. At $\rho=\rho_{\Lambda}, \Lambda$-hyperrons appear, and with a further increase of density, other heavier hyperons make an 
M82 X-1 (1-Class Configurations)

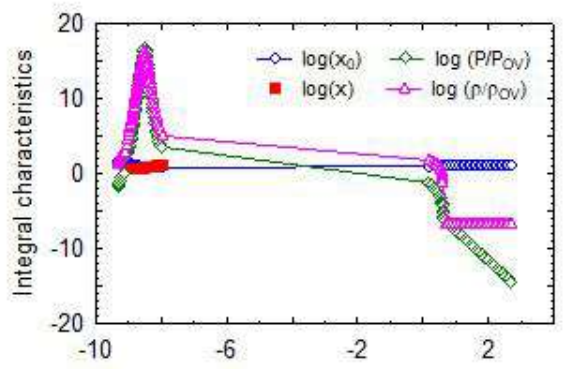

(a) $\log (\mathrm{r} / \mathrm{rov})$

M82 X-1 (1-Class Configurations)

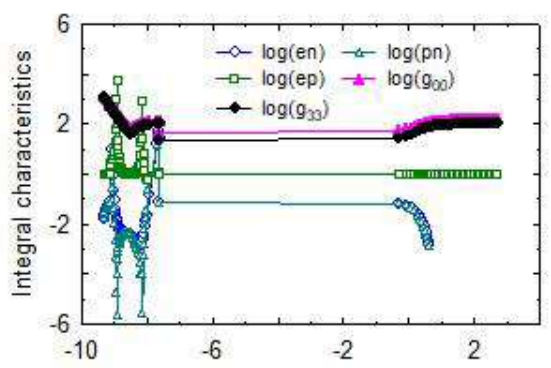

(c) $\log \left(\mathrm{r} / \mathrm{r}_{\mathrm{OV}}\right)$
M82 X-1 (II-Class Configurations)

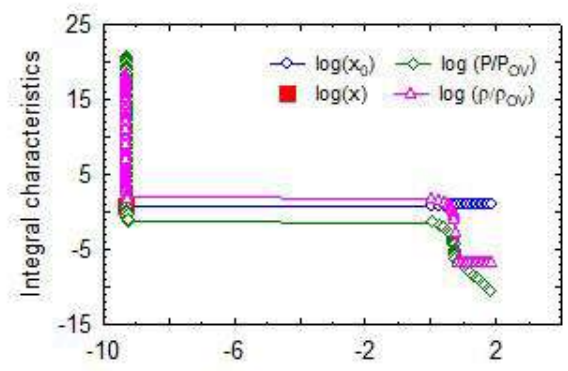

(b) $\log (\mathrm{r} / \mathrm{r}$ ov $)$

M82 X-1 (II-Class Configurations)

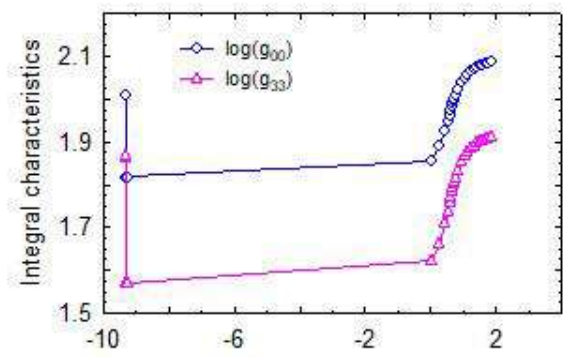

(d) $\log \left(r / r_{\circ}\right)$

Figure 18. The M82X-1: the radial profiles of pressure and density; gravitational $\left(x_{0}\right)$ - and ID $(x)$ potentials; $g_{00}$ and $g_{33}$ metric components; the ratios of electron-neutron (en), electron-proton (ep) and proton-neutron (pn) concentrations.

appearance altogether with . For baryons densities exceeding $5 \times 10^{16} \mathrm{gcm}^{-3}$, the following difficulties arise in the study of state equation: (i) very strong repulsive forces arise whose properties are not know well; (ii) the relative concentrations of various types of baryons can be strongly affected by the presence of higher hyperons having masses grater than that of the $\Xi$-hyperon. At still higher densities, $\pi$-mesons must also make their appearance as a Bose gas.

- The calculations made by Ambartsumian and Saakyan shown that the masses of equilibrium configurations of a non-rotating baryon ideal gas are of the ordder of a half of a solar mass, and that the radii reach out several kilometers. At small interparticle distances $\sim 0.4 \mathrm{fm}$ or less, strongly intense repulsive forces apparently come into action. The baryonic gas, therefore, can no longer be considered an ideal gas at baryon densities exceeding $1040 \mathrm{~cm}-3 \mathrm{~N}$ which corresponds to the short distances $l<0.5 \mathrm{fm}$. The masses of the degenerate configurations calculated for a real Fermi gas of baryons under the assumption that repulsive forces are active between the baryons, are appreciably larger than the masses of configurations of an ideal gas. The sizes and masses of the outer regions of a baryon star, i.e. neutron or proton-electron layers, comprise a small part of the total mass and size of the star at fairly high central densities. The bulk of the star's mass in those cases goes into the hyperon core.

- The study of the relationship between stellar parameters and total number of baryons in the star, and the dependence of that number on the density value at the center are problems of heightened interest. The configuration of lesser mass will be the more stable. A large mass exhibits a high density at the center. This must signify a large gravitational mass defect, in consequence of which the total mass of the configuration should be smaller in this case. The gravitational mass defect $\triangle M=n m_{H}-M$ of baryon stars has been investigated. Reviewing notations $M$ is the mass of the star, $n$ is the number of baryons present, and $m_{H}$ is the mass of hydrogen atom. It is shown that the configuration with 
M82 X-1 (1-Class Configurations)

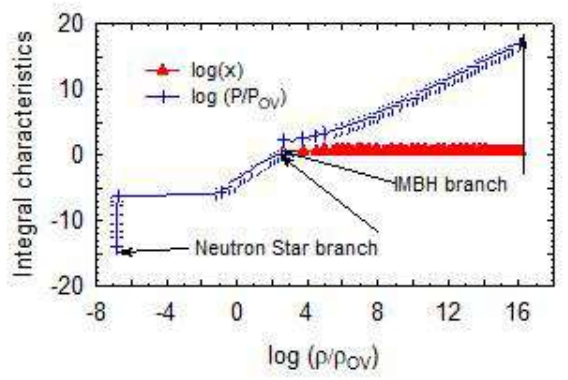

IC 467 (1-Class Configurations)

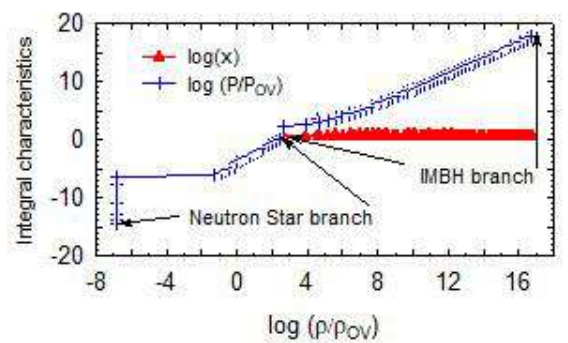

M82 X-1 (II-Class Configurations)

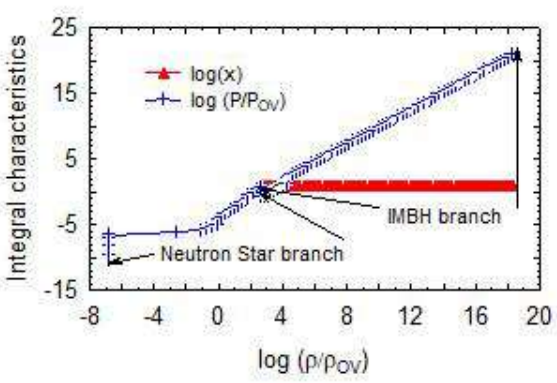

IC 467 (II-Class Configurations)

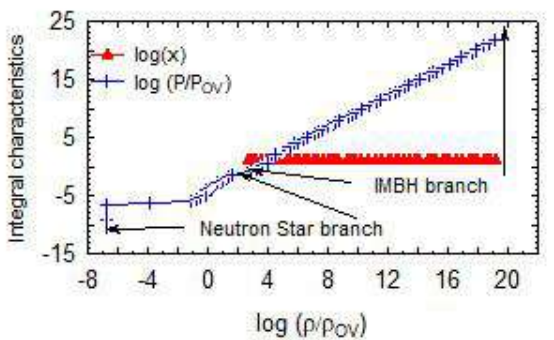

Figure 19. The state equations for M82 X-1 and IC 467.

$t_{n}(0) \lesssim 2.4$ is absolutely stable in the case of ideal gas, while the configuration with $t_{n}(0) \lesssim 2.9$ is absolutely stable in the case of real gas. The rest of configurations are either metastable or unstable. There is one intriguing feature in the unstable branches, namely that for $t_{n}(0) \gtrsim 4.2$ in the case of ideal gas and for $t_{n}(0) \gtrsim 3.15$ in the case of real gas, the binding energy $\triangle M$ becomes negative. Such states will be less stable than the others.

- A more detailed analysis of the absolute gravitational mass defect, $\triangle M$, of neutron configurations is of particular interest to astrophysicists. The computations of baryon configurations have demonstrated that when central densities rise above a certain value $\rho_{1}(0)$ the absolute mass defect changes sign - it becomes negative. The value of $\rho_{1}(0)$ depends on the form of the equation of state for the baryon gas used in the computations. In models with a real baryon gas $\rho_{1}(0)$ has a lesser value than in models with an ideal gas. This can be attributed to the fact that in the case of a real gas, at densities greater than nuclear, the nuclear forces of repulsion between baryons is played important role. The energy associated with one gram of star matter is an order of magnitude greater than the corresponding energy released in thermonuclear reactions in the combustion of hydrogen. It is important to note that the binding energy of each particle in a star is negative, so that the particles cannot escape individually to infinity. The escape of a certain number of baryons from a star requires the addition of supplementary energy to the remaining configuration from the outside. For this reason it cannot occur spontaneously. This means that transition of the system to a more stable state can occur only under the influence of very great perturbations. In this case expansion will occur, accompanied by heating of the celestial body. The corresponding transition will have the character of a cosmic explosion. These arguments concerning the fate of baryon configurations with an anomalous absolute mass defect were first presented by Ambartsumian and Saakyan. The configurations with an anomalous value of the absolute mass defect should be of some importance to Ambartsumian's cosmogony. In order to relate the above considerations on the behavior of baryon configurations with an anomalous value of the absolute mass defect to the concept mentioned, it is necessary to construct 
models of superdense prestellar bodies with masses of a much greater order of magnitude than the solar mass. This would solve in principle the problem of 'superdense' cosmogony. However, the construction of physical models of continuous superdense prestellar bodies of great mass involves difficulties. The solution of these difficulties may possibly involve the consideration of nonstationary and nonequilibrium models.

- We briefly review the observable signature and computational efforts of growth and merging phenomena of astrophysical black holes. We examine the meaning, and assess the validity of such properties within theoretical framework of PMBHs. We provide a discussion of some key objectives with the analysis aimed at clarifying the current situation of the subject. It is argued that such exotic hypothetical behaviors seem nowhere near true if one applies the PMBH. We briefly re-examined (critically) the past and present of the GR and PMBHs, and attempted to chart the future of the subject.

- A principle feature that makes general relativity (GR) distinctively different from other field theories is the occurrence of curvature singularities in spacetime. The singularities lead to regions of the universe that cannot be observed. This causes an observer's inability to access the degrees of freedom that are hidden beyond the horizon which, in turn, leads to thermodynamical behavior of BHs. Notwithstanding, much remarkably efforts have been made in understanding of BH physics, many important issues still remain unresolved and, thus, a situation is unclear, than described so far. The astrophysical significance of the issue, and the importance of considering the gravitational collapse of a matter cloud within the framework of the GR theory, with reasonable physical properties for the matter included, stems from the fact that GR predicts that a star more massive than about five to eight times the mass of the Sun, cannot stabilize to a neutron star final state at the end of its life cycle. It must collapse continually under the force of its own gravity on exhausting its internal nuclear fuel, and there are no known forces of nature that would halt such a collapse. General relativity predicts that such a star must then terminate into a spacetime singularity where densities and spacetime curvatures blow up and the physical conditions are extreme. The estimates on the mass limit for a star in order to collapse, of course, are indefinitely vary depending on different models for the star's interior and equation of state for matter at very high densities. One of the most important open issues in the theory and astrophysical applications of modern day $\mathrm{BH}$ and gravitation physics is that of the Roger Penrose's Cosmic Censorship Despite the past four decades of serious efforts, we do not have as yet available any proof or even any mathematically precise formulation of the cosmic censorship hypothesis, which is at the basis of much of the modern theory and astrophysical applications of BHs today. In recent years, a wide variety of gravitational collapse models have been discovered where exact analytical calculations have meanwhile shown that mass concentrations collapsing under their own weight will no longer form BHs as collapse endstate, rather naked singularities, except for configurations of highest symmetry which are, however, of measure zero among all initial data. By this, even the theoretical existence of $\mathrm{BHs}$ is no longer justified. Some conceptual problems of the energy-momentum conservation laws of gravitational interacting fields, the localization of energy of gravitation waves, the role of singularities, and also severe problems involved in quantum gravity are still plaguing GR. Within respect to standard models, a hard look at the PMBH physics reveals at least severe principle difficulties. Although no results on BH thermodynamics have been subject to any experimental or observational tests, the attempts of theoretical interpretation of the $\mathrm{BH}$ thermodynamics provide a basis for further research and speculation on the nature of its quantum gravitational origin. The efforts to understand the mysterious statistical mechanical properties of BHs has led to many speculations about their quantum gravity origin. In particular, one cannot answer which region of AdS is responsible to particular information in the dual CFT. There is also an essential discrepancy between the entanglement entropy and the $\mathrm{BH}$ entropy, that the entanglement entropy is proportional to the number of matter fields, while the BH entropy is not. The former includes ultraviolet divergences as opposed to the latter. So, due to existing discrepancies and absence of distinctive observationally tested predictions, there is no convincing reason to rely on the string theory in its present form. This then ruptures the familiar BH entropy illusion which has insufficient dimensions. Thus we conclude 
that PBHM, at least at its current state of development, is quite incapable of making predictions on growth and merging properties of the astrophysical BHs, because of the nasty inherent appearance of $\mathrm{BH}$ singularities, and that if the infinite collapse to the singularity inside the $\mathrm{BH}$ is accepted as a legitimate feature of Nature. Only a true internal solution was able to give a reliable information on the thermodynamic behavior and entropy of $\mathrm{BH}$ if, and only if, it is known deep within the $\mathrm{BH}$.

- To fill the void which the standard PMBH presents, and to innovate the solution to alluded problems, we have recounted some of the highlights behind of MTBH, whereas the infra-structures will inevitably be accommodated inside the EH. To start with, we discuss the proposed general theory of distortion of space-time continuum at huge energies (respectively, at short distances $<0.4 \mathrm{fm}$ ). As a corollary, in particular, DSTC leads to modified gravitational theory, underlying the MTBH. Much use has been made in proposed gravitation theory of the language of fundamental geometric structure - a distortion gauge induced fiber-bundle, incorporating with the spacetime deformation/distortionframework. The general gauge principle innovates the solution to the problems of standard Riemannian (and its extensions) space interacting quantum field theory. Involving a drastic revision of the role of gauge fields in the physical concept of curved geometry, this theory generalizes the standard gauge scheme. The nonlinear realization technique or the method of phenomenological Lagrangians provides a way to determine the transformation properties of fields defined on the quotient space. We treat the distortion group and its stationary subgroup, respectively, as the dynamical group and its algebraic subgroup. The fundamental field is distortion gauge field (a) and, thus, all the fundamental gravitational structures in fact - the metric as much as the coframes and connections - acquire a distortion-gauge induced theoretical interpretation. We study the geometrical structure of the space of parameters in terms of Cartan's calculus of exterior forms and derive the Maurer-Cartan structure equations, where the distortion fields (a) are treated as the Goldstone fields. The available solar system observational verifications offer many opportunities to improve tests of relativistic gravity. Besides, the GGP explores the most important processes of spontaneous breaking of gravitation gauge symmetry and rearrangement of vacuum state. Spontaneous symmetry breaking can be achieved by introducing the neutral complex scalar Higgs field. Minimization of the vacuum energy fixes the non-vanishing vacuum expectation value, which spontaneously breaks the theory, leaving the U(1)d subgroup intact, i.e. leaving one Goldstone boson. The left Goldstone boson is gauged away from the scalar sector, but it essentially reappears in the gauge sector providing the longitudinally polarized spin state of one of gauge bosons that acquires mass through its coupling to Higgs scalar. The two neutral gauge bosons were mixed to form two physical orthogonal states of the massless component of distortion field, which is responsible for gravitational interactions, and its massive component, which is responsible for the ID-regime. Hence, a substantial change of the properties of the spacetime continuum besides the curvature may arise at huge energies. The theory is renormalizable, because gauge invariance gives conservation of charge, also ensures the cancelation of quantum corrections that would otherwise result in infinitely large amplitudes.

- To innovate the solution to aforementioned problems, we advocate with alternative proposal by utilizing the MTBH, which has explored a novel aspects expected from considerable change of properties of space-time continuum at spontaneous breaking of gravitation gauge symmetry far above nuclear density. The MTBH is a first-principles treatment of a fundamental superdense protomatter physics, but it also has an actual physical realization of Ambartsumian's fundamental vision. It may shed further light upon the growth and merging phenomena of astrophysical BHs.

The MTBH is an extension of PBHM and rather completes it by exploring the most important processes of spontaneous breaking of gravitation gauge symmetry at huge energies, and thereof for that of rearrangement of vacuum state. Whereas a significant change of properties of space-time continuum (ID-regime), arises simultaneously with the strong gravity. This manifests its virtues below the ID-threshold length $(0.4 \mathrm{fm})$, yielding the transformations of Poincar'e generators of translations. Accordingly, a matter found in ID-region of spacetime continuum is undergone phase transition of II-type, i.e., each particle goes off from the mass shell. Hence, a shift of mass and energy-momentum 
spectra occurs upwards along the energy scale. The thermodynamics of a resulting matter, so-called proto-matter, is drastically differed from the thermodynamics of strongly compressed ordinary matter. The energy density and internal pressure have sharply increased in the central region of configuration, proportional to gravitational forces of compression up to $\sim 10^{25}$ order of magnitudes with respect to corresponding central values of neutron star. In the resulting so-called proto-matter, the pressure becomes dominant over gravitational force at very short distances when matter falls into central singularity as the collapse proceeds and, thus, it halts the infinite collapse. This supplies a powerful pathway to form a the equilibrium superdense proto-matter core. The stable equilibrium holds for outward layers too. This counteracts the collapse and equilibrium condition remains valid even for the masses up to $\sim 10^{10} M_{\odot}$. As a corollary, this theory has smeared out the central singularities of $\mathrm{BH}$ at very strong gravitational fields. One of the most remarkable drawback of MTBH is the fact that instead of infinite collapse and central singularity, an inevitable end product of the evolution of massive object is the stable SPC, where static observers exist. It will ultimately circumvent a principle problem of an observer's inability to access the degrees of freedom that are hidden beyond the horizon, and a necessity to assign the elusive entropy to BH. This in somehow or other implies that a physical entropy is assigned to SPC as a measure of the large number of thermodynamical real microstates of proto-matter, which is compatible with a concept of ergodicity. This may shed further light upon the growth and merging phenomena of astrophysical BHs, that are in evidence throughout the universe.

The ID mechanism accommodates the highest energy scale in central SPC. Encapsulated in an entire set of equations of equilibrium configuration, the SPC is a robust structure that has stood the tests of the most rigorous theoretical scrutinies of a stability. It also helps to reassure us that the stable equilibrium holds in outward layers too. In this way, an accumulation of matter is allowed about SPC. Moreover, above nuclear density, the SPC always resides inside the event horizon, therefore it could be observed only in presence of accreting matter. The external physics of accretion onto the SPC in first half of its lifetime is identical to the processes in phenomenological BH models. In other words, there is no observable difference between the gravitational field of SPC and Schwarzschild BH, so that the observable signature of BHs available in literature is of direct relevance for the SPC-configurations too. But MTBH manifests its virtue when one looks for the internal physics, accounting for growth and merging behavior of BHs. A crucial point of the MTBH is that a central singularity cannot occur, which is now replaced by SPC, where the static observers are existed. The seed BH might grow up driven by the accretion of outside matter when it was getting most of its mass.

- One of the achievements of contemporary observational astrophysics is the development of a quite detailed study of the physical properties of growth and merging phenomena of astrophysical black holes, even at its earliest stages. But even thanks to the fruitful interplay between the astronomical observations, the theoretical and computational analysis, the scientific situation is, in fact, more inconsistent to day. The growth of accreting supermassive black hole seeds and their neutrino radiation are found to be a common phenomena in the AGNs. We further expose the assertions made in the framework of microscopic theory of black hole via reviewing the mass assembly history of 377 plausible accreting supermassive black hole seeds. After the numerous reiterating integrations of the state equations of SPC-configurations, we compute their intermediate seed masses, $M_{B H}^{\text {Seed }}$, PRTs, initial redshifts, $z^{\text {Seed }}$ and neutrino fluxes. All the results are presented in the Tables 1-5. The Fig. 8 gives the intermediate seed masses $M_{B H}^{S e e d}$ versus the present masses $M_{B H} / M_{\odot}$ of 337 black holes, on logarithmic scales. In accord, the AGNs are favored as promising pure UHE neutrino sources. Such neutrinos may reveal clues on the puzzle of origin of UHE cosmic-rays. We regard the considered black holes only as the potential neutrino sources. The obtained results, however, may suffer and that would be underestimated if not all 377 live black holes in the $M_{B H} / M_{\odot} \simeq 1.1 \times 10^{6}$ to $1.3 \times 10^{10}$ mass range at present reside in final stage of their growth, when the proto-matter disk driven by accretion has reached out the event horizon.

- The analysis of periodic source M82X-2 in this paper goes contrary to the conventional wisdom. Namely, putting apart the discussion of inherent problems of the mass scaling of the black holes in 
ULXs, we have focused on black hole rather than typical accreting pulsar models. It is, of course, impossible to explain the pulsed high luminosity in the framework of widely accepted conventional phenomenological black hole model because black holes do not radiate, also the spinning black holes are axisymmetric and have no internal structure on which to attach a periodic emitter. The new conceptual element of the implications of the framework of MTBH in tackling this problem is noteworthy. The MTBH explores the most important processes of spontaneous breaking of gravitation gauge symmetry and rearrangement of vacuum state at huge energies, making room for growth and merging behavior of black holes. We assume a source of flashes M82X-2 is being SIMBH, resided in the final stage of growth. If this were the case, eventually, a thin co-spinning proto-matter disk driven by accretion would be formed around the spinning proto-matter core, tilted from the plane of accretion on a definite angle $\delta$ towards the equator. It has reached out the edge of the event horizon where a metric singularity inevitably disappears. The energy is carried away then from event horizon through a thin belt area to outside world by both the ultra-high energy neutrinos produced in the superdense proto-matter medium, and the thermal defuse blackbody radiation released from the outer surface layers of ordinary matter of spinning SPC and co-spinning proto-matter disk. All of the rotational energy of SIMBH is dissipated as thermal defuse blackbody X-ray radiation to outside world. The manner in which we led the reader to the result, it should not come as a surprise. As M82X-2 spins, we see pulses because of the axial tilt or obliquity. That is to say, the M82X-2 is the emitter of both persistent continuous broad X-ray radiation and pulsating X-rays. We derive the general profiles of pulsed luminosity and X-ray flux of M82X-2. Thus, M82X-2 indeed releases $99.59 \%$ of its radiative energy predominantly in the X-ray bandpass of $0.3-30 \mathrm{keV}$. Since there is not enough information to test the theory, i.e. in the absence of direct mass-function measurements from phase-resolved optical spectroscopy, we still have to rely on X-ray spectral and timing modeling and other indirect clues and, thus, the resulting theoretical model necessarily includes a number of poorly known parameters. We give a quantitative account of all the energetics, a potential dynamical mass scaling and orbital parameters of the semi-detached X-ray binary containing primary M82X-2 and the secondary massive O/B-type donor star, accreting through Roche-lobe overflow. These results have a heuristic value but are far from rigorous. At hard look, the position angles can be adjusted from rigorous comparison with the behavior of observed pulsed light curve of M82X-2, which will be discussed in a future publication.

- We supplement our previous investigation by more rigorous analytical treatment of rotating SPC. We analytically treat the microscopic model of stationary and axisymmetric rotating black hole. The non-spinning SPC is static and spherically symmetric. Therefore, we need to be clear about more general geometry which can describe rotating axisymmetric SPC. A rotating massive configuration drags space and time around with it (non-Newtonian gravitational effect). The local inertial frames are dragged by the rotation of the gravitational field, i.e. a gyroscope orbiting near the configuration will be dragged along with the rapidly rotating configuration. This is probably the most remarkable feature that could serve as a link with the general description of spacetime. Beside the geodetic procession, a spin of the body produces in addition the Lense-Thirring procession. The most remarkable feature of microscopic model of a rotating black hole is that, in earlier part of its lifetime, the external physics outside of outer oblate event horizon of accretion onto a black hole is identical to the processes in Kerr's model. But, there is also a crucial difference between internal physics of Kerr and microscopic rotating black hole models. That is, a central ring singularity of the Kerr black hole cannot occur, which is now replaced by finite though unbelievably extreme conditions held in the central part of rotating SPC, where the static observers exist. This has then made room for growth and merging properties of black holes. The rotating SPC necessarily introduces essential corrections to the astrophysical calculations of previous models. This was accounted, for particular example, in X-ray pulsations from M82X-2.

- For a broad range of parameters, the numerous reiterating integrations of the entire set of equations of equilibrium SPC-configurations allow to compute all the essential physical characteristics of 137 IMBH-candidates. The BH mass is an important parameter in this study. Of course, there are still large uncertainties in mass estimates collected from the literature of all the observational 
evidence for 137 IMBH-candidates. However, for brevity reasons to save space, we retain rather a concrete proposal to proceed in relatively simple way. That is, we choose the computed mass values of the IMBH-candidates to be matched the mean values of observational mass estimates of corresponding objects. It is sensible to do so, especially since we have already reflected upon the matter. We comment on the observational mass uncertainties for some of these objects, and their validity or the confidence. In the same time, for overall details regarding this issue, we invite the interested reader to consult further the papers cited. We present the figures, which clearly highlight a main difference between both classes of discussed configurations.

Reflecting upon the results so far obtained, we demonstrate how much should be gained for high energy astrophysics by further study of the fundamental paths laid by Ambartsumian. That is, developing Ambartsumian's ideas, we consequently have arrived to a new physical perception of space-time geometry, namely the general theory of DSTC at huge energies (at short distances $<0.4 \mathrm{fm}$ ). It leads to modified gravitational theory as a corollary of the particular case of spacetime deformation/distortion framework. This theory, in turn, underlies the MTBH, which has smeared out the central singularities of BHs, and makes room for their growth and merging behavior. The MTBH is a first-principles treatment of a fundamental superdense protomatter physics, but it also has an actual physical realization of Ambartsumian's fundamental vision, which enables an insight to key puzzles of ultra-high energy astrophysics. However, it should be emphasized that the key to our construction procedure is widely based on the premises of our experience of accretion physics. Therefore, what we have presented here has all the vices and virtues of the classical scenario of runaway core collapse which has always been a matter of uncertainties and controversies. Nevertheless, we caution that these entire constructions will be valid as well in the case if some hitherto unknown yet mechanism in Nature will in somehow or other way produce the superdense proto-matter, away from the accretion physics as Ambartsumyan has believed.

\section{References}

Ambartsumian V. A., 1947, The Evolution of Stars and Astrophysics, (in Russian). Armenian SSR Academy of Sciences Press, Yerevan

Ambartsumian V. A., 1949, Stellar Associations, Astronomicheskii Zhurnal, 26(1), 3

Ambartsumian V. A., 1954, The Phenomenon of Continuous Emission and Sources of Stellar Energy, ComBAO, 13, 3

Ambartsumian V. A., 1955, Stellar systems of positive total energy, The Observatory, 75, 72

Ambartsumian V. A., 1958a, in On the Evolution of Galaxies, XI Solvay Conf., Brussel. p. 241

Ambartsumian V. A., 1958b, On the Evolution of Galaxies, Izvest. Akad. Nauk ArmSSR, 11(5), 9

Ambartsumian V. A., 1958c, On the Problem of the Mechanism of the Origin of Stars in Stellar Associations, Reviews of Modern Physics, 30, Issue 3, 944

Ambartsumian V. A., 1960, On the Evolution of Stellar Systems, George Darwin Lectures, Quarterly Journal of the Royal Astronomical Society, 1, 152

Ambartsumian V. A., 1961, Instability phenomena in systems of galaxies, Astronomical Journal, 66, 536

Ambartsumian V. A., 1962, Problems of Extra-Galactic Research, Transactions of the International Astronomical Union, 11B, 145

Ambartsumian V. A., 1965, The Nuclei of Galaxies and Their Activity, The Structure and Evolution of Galaxies. Library of Congress Catalog Card No. 65-26979. Interscience Publishers, a division of John Wiley \&Sons, Ltd., London

Ambartsumian V. A., 1966, Some remarks on the nature of the nuclei of galaxies, Transactions of the International Astronomical Union, 12B, 578

Ambartsumian V. A., 1968a, Galaxies and galactic evolution, "The Earth in the Universe". Israel Program for Scientific Translations, Jerusalem

Ambartsumian V. A., 1968b. The Pubblishing House of the Academy of Sciences of Armenian SSR, Yerevan, p. 521

Ambartsumian V. A., 1971, Fuors, Astrophysics, SSSR, 7, Issue 4, 557

Ambartsumian V. A., 1984, The questions of superdense celestial bodies, (in Russian). State Univ. press, Yerevan 
Ambartsumian's vision and further insight to key puzzles of ultra-high energy astrophysics

Ambartsumian V. A., 1985, On the dynamics of open clusters, Dynamics of Star Clusters; IAU Symposium, May 29-June 1, 1984. Dordrecht, D. Reidel Publishing Co., Princeton, NJ

Ambartsumian V. A., Saakyan G. S., 1960, The Degenerate Superdense Gas of Elementary Particles, Astronomicheskii Zhurnal, 37,193

Ambartsumian V. A., Saakyan G. S., 1961a, On Equilibrium Configurations of Superdense Degenerate Gas Masses, Astronomicheskii Zhurnal, 38, 785

Ambartsumian V. A., Saakyan G. S., 1961b, Internal Structure of Hyperon Configurations of Stellar Masses, Astronomicheskii Zhurnal, 38, 1016

Ambartsumian V. A., Saakyan G. S., 1965, The Mass Defect of Baryon Stars, Astrophysics, 1, Issue 1, 1

Baade W., Zwicky F., 1934, On super-nova, Proc. Nat. Acad. Sci., 20, 2599

Bassani L., Dean A. I., S S., 1983a, Preprint Univ. Southampton, Dept. Phys., Southampton, S09 5NH, UK

Bassani L., Dean A. I., S S., 1983b, Super-Eddington luminosity characteristics of active galactic nuclei, Astron. Astrophys., 125, 52

Cameron A. G. W., 1950, Neutron star models, Astrophys. J., 130, 884

Collaboration I., 2018, Multimessenger observations of a flaring blazar coincident with high-energy neutrino, IceCube-170922A, Science

Joshi P. S., Malafarina D., 2013, Instability of black hole formation under small pressure perturbations, Gen. Rel. Grav., 45, 305

Landau L., 1932, Neutron Star Structure and Equation of State, Phizik Z. Sowietunion, 1, 285

Linsley J., 1963, "Primary cosmic-rays of energy $10^{17}$ to $10^{20} \mathrm{ev,} \mathrm{the} \mathrm{energy} \mathrm{spectrum} \mathrm{and} \mathrm{arrival} \mathrm{directions",} \mathrm{Proc.} \mathrm{from} \mathrm{the} 8$ th International Cosmic Ray Conference, 4, 77

Nambu Y., Jona-Lasinio G., 1961, Dynamical model of elementary particles based on an analogy with superconductivity, Phys. Rev., 122,345

Oppenheimer J. R., Volkoff G. M., 1939, On massive neutron cores, Phys. Rev., 55, 374

Ter-Kazarian G., 1986, Distortion of the Space-Time, Textbook:"Selected Questions of Theoretical and Mathematical Physics". Vol. N5322-B86, No1, 62-146, VINITI, Moscow, Russia

Ter-Kazarian G., 1989a, Supermassive Equilibrium Configurations of Superdense Matter, Akademiia Nauk SSSR, Doklady (ISSN 0002-3264), In Russian, 309, No.1, 97-101

Ter-Kazarian G., 1989b, On Study of Equilibrium Configuration of Protomatter, Part I, Astrofizika, 31, Issue 2, 345-358

Ter-Kazarian G., 1989c, The Theory of Distortion of Space-Time Continuum, ComBAO, 62, 1-123

Ter-Kazarian G., 1990, On Study of Equilibrium Configuration of Protomatter, Part II, Astrofizika, 33, 143-153

Ter-Kazarian G., 1991, On the study of internal structure of superdense celestial bodies. Astrofizika, 35, Issue 2,3, 335-372

Ter-Kazarian G., 1992, An Introduction to the Theory of Internal Structure of Supermassive Compact Celestial Bodies, Astrophysics \& Space Sci., 194, No1, 1-129

Ter-Kazarian G., 1997, Gravitation Gauge Group, Nuovo Cimento, 112B, No 6, 825-838

Ter-Kazarian G., 2001, Protomatter and EHE C.R., Japan Phys. Soc. Jpn., 70, S. B., 84-98

Ter-Kazarian G., 2010, Gravitation and inertia; a rearrangement of vacuum in gravity; Astrophysics \& Space Sci., 327, 91-109, arxiv: $1001.2990[\mathrm{gr}, \mathrm{qc}]$

Ter-Kazarian G., 2011, Two-step spacetime deformation-induced dynamical torsion, Class. Quantum Grav., 28, 055003 (19pp); stacks.iop.org/CQG/28/000000; arxiv:1102.24911[gr,qc]

Ter-Kazarian G., 2012, Spacetime deformation-induced inertia effects, Advances in Mathematical Physics, Article ID 692030, 1-41, http://dx.doi.org/10.1155/2012/692030

Ter-Kazarian G., 2014, Ultra-high energy neutrino fluxes from supermassive AGN black holes, Astrophys. \& Space Sci., 349, 919-938

Ter-Kazarian G., 2015, Modified Theories of Gravitation behind the Spacetime Deformation, Physics Research International, 2015, Article ID 152846, 1-19, http://dx.doi.org/10.1155/2015/152846

Ter-Kazarian G., 2016a, Rotating black holes in microscopic theory: the implications for periodic source M82X-2, Advances in Astrophysics, 1, issue 3, 201-223

Ter-Kazarian G., 2016b, On the Physical Nature of the Source of Ultraluminous X-ray Pulsations, Astrophys. \& Space Sci., 361, issue 1, 20pp, DOI $10.1007 / \mathrm{s} 10509$ 
Ter-Kazarian G., 2021a, Growth and merging phenomena of black holes: observational, computational and theoretical efforts, ComBAO, 68, issue 1, pp. 56-74, https://doi.org/10.52526/25792776

Ter-Kazarian G., 2021b, A new look at some aspects of geometry, particle physics, inertia, radiation and cosmology, ComBAO, 68, issue 2, pp. ??, https://doi.org/10.52526/25792776

Ter-Kazarian G., Sargsyan L., 2013, Signature of plausible accreting supermassive black holes in Mrk 261/262 and Mrk266, Advances in Astronomy, 2013, Article ID 710906, http://dx.doi.org/10.1155/2013/710906

Ter-Kazarian G., Shidhani S., 2017, Plausible Intermediate Mass Black Hole from M82X-2 X-Ray Pulsations, Advances in Astrophysics, 2 , issue $3,162-183$

Ter-Kazarian G., Shidhani S., 2019, A study of 137 intermediate mass black hole candidates, Astrophys. Space Sci., 364, Issue 10, article id. $165,23 \mathrm{pp}$

Ter-Kazarian G., Yerknapetian K., 1995, On the Internal Structure of Supermassive Compact Celestial Bodies, Astrofizika, 38, No4, 659-666

Ter-Kazarian G., Khachatryan V., Yerknapetyan K., 2003, Neutrino Cooling and Fueling at Disk Accretion Onto the SPC in AGN, and Origin of EHE G.R.s, "The Universe Viewed in Gamma-rays", ICRR, University of Tokyo. Universal Academy Press, Inc., BR-Hongo-5 Bldg., 6-16-2 Hongo Bunkyo-ku, Tokyo, Japan

Ter-Kazarian G., Terzyan Y., Khachikian E. e. a., 2006, On Multi-Nuclei Features of Some Markarian Galaxies, Monthly Notice of Royal Astron. Soc., 368, 461-470

Ter-Kazarian G., Shidhani S., Sargsyan L., 2007, Neutrino Radiation of the AGN Black Holes, Astrophys. \& Space Sci., 310, issue $2,93-110$

Zel'dovich Y. B., 1959, Soviet Physics- JETP, 10, 409

Zel'dovich Y. B., 1962, Static solutions with energy excess in general relativity, Soviet Physics- JETP, 42,1667

Zwicky F., 1938, On Collapsed Neutron Stars, Astrophys.J., 88, 522 